\title{
Taxonomy and phylogeny of the Old World jumping plant-louse genus Paurocephala (Insecta, Hemiptera, Psylloidea)
}

\author{
D. MIFSUD $\dagger$ and D. BURCKHARDT
}

Naturhistorisches Museum, Augustinergasse 2, CH-4001 Basel, Switzerland; e-mail: daniel.burckhardt@unibas.ch

(Accepted 15 February 2001)

Fifty-one species are recognized in the genus Paurocephala, with an additional 14 species which remain unnamed. Thirty-five species are described as new, and five species are synonymized: $P$. pumilae and $P$. zhejiangensis with $P$. chonchaiensis, $P$. debregeasiae with $P$. sauteri, and $P$. guangxiensis and $P$. tremae with $P$. trematos. Two varieties, $P$. psylloptera maculipennis and $P$. psylloptera setifera, are raised to species level. $P$. bifasciata is reinstated from former synonymy with $P$. chonchaiensis, and Anomoterga kleinhofiae is transferred back to Paurocephala. Lectotypes are designated for $P$. brevicephala, $P$. chonchaiensis, $P$. psylloptera, $P$. sauteri, $P$. setifera and $P$. wilderi. The four New World species of Paurocephala are not considered to be congeneric with the Old World species and will be transferred to Diclidophlebia. Two South African species, $P$. bicarinata and $P$. hottentotti, are removed from Paurocephala and have to be accommodated in a new genus in the Diaphorininae. Thus Paurocephala is an Old World genus with nine Afrotropical and 42 Indo-Australian described species. Keys for the identification of adults and fifth instar larvae are provided. All 51 named species are diagnosed and illustrated, and information is given on distribution and host plants. Based on two cladistic analyses, one using adult characters only, and one using both adult and larval characters, four monophyletic species groups are recognized. In both analyses a basal group of 10 species, the brevicephala-group, forms the sister group to all other Paurocephala spp. The Afrotropical species are monophyletic and, together with one Oriental species constitute the gossypiigroup. In the analysis with adult characters only, the gossypii-group is the sister taxon of the kleinhofiae-group and, both together, are the sister group of the psylloptera-group, the largest species group with 27 species. In the analysis of adult and larval characters, the relationships between the last three species groups are not resolved. The result of the phylogenetic analysis confirms the synonymy of the subgenus Thoracocorna with Paurocephala. Known host plants of Paurocephala spp. belong to the Malviflorae with the exception of the Afrotropical $P$. insolita which develops on Theiflorae (Theales, Clusiaceae). The brevicephala-, kleinhofiae- and gossypii-groups are associated with hosts of the order Malvales (Malvaceae and Sterculiaceae), whereas the psylloptera-group is with Urticales (Urticaceae, Moraceae and Ulmaceae).

KeYwords: Paurocephala, Hemiptera, Psylloidea, taxonomy, Urticales, Malvales, cladistics, phylogeny.

†Present address: Department of Agriculture, Research and Development Centre, Ghammieri, Marsa, Malta; e-mail: davmif@hotmail.com 


\section{Introduction}

Among phytophagous Hemiptera, jumping plant-lice or psylloids are particularly interesting for their highly specialized host requirements. Individual psylloid species can usually complete their larval development on only one or a few closely related host species. In addition to this, related psylloids are often associated with closely related plant taxa. Psylloids constitute, therefore, a promising taxon for testing co-evolutionary hypotheses of insects and their host plants (Burckhardt and Basset, 2000).

A prerequisite for studies of this kind is a sound taxonomic and phylogenetic base. Revisions published in the last 25 years (e.g. Hollis, 1976; Brown and Hodkinson, 1988; Hollis and Broomfield, 1989; Burckhardt and Basset, 2000) and extensive collections made in the tropics and South temperate regions show, however, that our knowledge on the diversity and phylogeny of jumping plant-lice is still fragmentary. The some 3000 described species represent probably much less than half the number of globally existing species. Although the current classification based on White and Hodkinson's (1985) analysis reflects better phylogenetic relationships within Psylloidea than traditional ones (Bekker-Migdisova, 1973), the monophyly of many groups remains questionable. One of these groups is the mainly tropical genus Paurocephala, which has been a continuing source of confusion.

Crawford (1914) erected the genus Paurocephala for P. psylloptera, a species that was collected on Ficus ulmifolia (Moraceae). He noted a resemblance to species of the genus Pauropsylla Rübsaamen, most of which are associated with Ficus spp., and thereby implying a close phylogenetic relationship. This produced a lot of subsequent confusion about concept and content of both genera, which continued until the 1970s. Also, in 1975, Mathur described species of Paurocephala, from the Indian subcontinent, stating that they closely agree with morphological characters of Pauropsylla. These two genera, which were treated as closely related and placed in the same tribe (Pauropsyllini), were re-examined by Hollis (1984). He placed the genus Paurocephala near Haplaphalara Uichanco and Moraniella Loginova, which confirmed the placement of Paurocephala in the Aphalaridae proposed by several authors (Vondráček, 1957; Heslop-Harrison, 1958; Klimaszewski, 1964). Pauropsylla, on the other hand, was redefined within the Triozidae (Hollis, 1984).

Vondráček (1952, 1957), Heslop-Harrison (1951) and Dobreanu and Manolache (1962) included the genus Camarotoscena Haupt as a subgenus of Paurocephala. The subgenus Thoracocorna was established for Paurocephala chonchaiensis Boselli (Klimaszewski, 1970) and later synonymized with Paurocephala (Loginova, 1972).

Crawford (1919) and Uichanco (1921) described new varieties for series which resembled $P$. psylloptera Crawford, but which were collected on different hosts. These varietal names were still used until recently (Navasero and Calilung, 1998). Other authors (Enderlein, 1914; Crawford, 1917; 1919; Uichanco, 1921; Boselli, 1929; Kuwayama, 1931), described and/or synonymized additional Oriental taxa within Paurocephala, without examining type material. The consequence of this was complete confusion. The important contribution of Yang et al. (1986) solved some of the confusion around P. psylloptera Crawford. They concluded that three different species were involved; one developing on Ficus, one on Trema and one on Morus, all three species having restricted geographical distributions. These results were based on host plant alternation and mating experiments, courtship behaviour, acoustic analysis and adult morphology. Li and Yang (1987) described five new species of Paurocephala from China, but failed to examine type material of previously described species. 
Pettey $(1924,1933)$ described two Paurocephala species from South Africa based on absence of genal processes and presence of pterostigma. Russell (1943, 1946) described two new Afrotropical species in Paurocephala; though similar, they differed substantially from the type species, most conspicuously in the presence of prominent peg-like setae on the inner surface of the paramere.

From the New World, Brown and Hodkinson (1988) described two Neotropical species, $P$. lanceomedia and $P$. paucivena. They suggested that the former was closely related to the Afrotropical species, differing in the absence of peg-like setae on the inner surface of the paramere and in wing venation. They did not comment on the relationships of $P$. paucivena Brown and Hodkinson, due to lack of males and antennal structures.

Burckhardt (1996) described two Paurocephala spp. from Paraguay. These two species differed from all other species included in Paurocephala in the distinctly short head, thickened antennal flagellum and presence of a small subapical rhinarium on segment 3. He suggested that two Asian groups of Paurocephala can be distinguished. One group has long and slender metatibiae, a long rostrum, antennae longer than head width and long female terminalia. The second group, consisting of $P$. brevicephala Crawford, $P$. kleinhofiae Uichanco, $P$. minuta Crawford and $P$. russellae Mathur, has short metatibiae and rostrum, antennae shorter than head width and short female genitalia, which are usually upturned apically. He also suggested that the Afrotropical species are intermediate between these two Asian groups.

The aim of the present work is to redefine the genus Paurocephala and its constituent species as well as to examine the phylogenetic relationships within the genus.

\section{Material and methods}

\section{Morphological terminology}

Morphological terminology follows mostly Ossiannilsson (1992). Hollis (1976) is followed in the usage of the terms 'spurs' and 'spines' in relation to the metatibia. The term 'claval fold' is used instead of 'claval suture' in the forewing. Forewing vein morphology and measurements are shown in figure 1A, B. Yang et al. (1986) is followed for the definition of the meracanthal length.

Measurements are given in $\mathrm{mm}$ and were made from slide-mounted specimens using an eye-piece graticule on the dissection microscope.

Following abbreviations are used in the descriptions, keys and measurement tables. Adult psylloid: HW, head width; AL, antenna length; F1, length of first antennal flagellomere; LB, labrum length; WL, forewing length; WW, forewing width; PT, pterostigma length; RL, Rs vein length of forewing; RC, length of line connecting apices of vein $\mathrm{Rs}$ and $\mathrm{Cu}_{1 \mathrm{a}}$ of forewing; a, length of line connecting base and apex of vein $\mathrm{M}_{1+2}$ of forewing; $b$, length of line connecting base and apex of vein $\mathbf{M}_{3+4}$ of forewing; $c$, length of line connecting apices of veins $\mathbf{M}_{1+2}$ and $\mathbf{M}_{3+4}$ of forewing; $\mathrm{d}$, length of line connecting apices of vein $\mathrm{Cu}_{1 \mathrm{a}}$ and $\mathrm{Cu}_{1 \mathrm{~b}}$ of forewing; $\mathrm{e}$, length of widest perpendicular distance to $\mathrm{d}$ in cell $\mathrm{cu}_{1} ; \mathrm{ML}$, metatibial length; MC, meracanthus length; MP, male proctiger length; PL, paramere length; DL, length of distal segment of aedeagus; FP, female proctiger length; CL, circumanal ring length; SL, female subgenital plate length. Fifth instar larva: AL, antenna length; FL, forewing-pad length; BL, body length; BW, body width; CL, caudal plate length; $\mathrm{CW}$, caudal plate width. 


\section{Material depositories}

Material is conserved dry, in $70 \%$ ethanol or is mounted on microscopical slides. Specimens were examined or are cited from following depositories: BAUC, Beijing Agricultural University, Beijing, China; BMNH, The Natural History Museum, London, UK; BPBM, Bernice P. Bishop Museum, Honolulu, Hawaii, USA; DEIC, Deutsches Entomologisches Institut, Eberswalde Finow, Germany; EIHU, Entomological Institute, Hokkaido University, Sapporo, Japan; IFRI, Indian Forest Research Institute, Dehra Dun, India; LCMI, Entomology Research Institute, Loyola College, Madras, India; MHNG, Muséum d'histoire naturelle, Geneva, Switzerland; MRAC, Musée Royal de l'Afrique Centrale, Tervuren, Belgium; NCHU, National Chung Hsing University, Taiwan; NHMB, Naturhistorisches Museum, Basel, Switzerland; OMNH, Osaka Museum of Natural History, Osaka, Japan; TULE, Tokyo University of Agriculture, Laboratory of Entomology, Japan; UNEI, Dipartimento di Entomologia e Zoologia Agraria del Università, Portici, Naples, Italy; UPPC, Department of Entomology, University of the Philippines, Laguna, Philippines; USNM, National Museum of Natural History, Washington, DC, USA (psylloid collection deposited in the USDA, Beltsville, MD, USA).

\section{Phylogenetic analyses}

The cladistic analyses were performed with WINCLADA (Nixon, 1999) and Hennig86 (Farris, 1988), using the $\mathrm{mh}$ and bb* search options. Diclidophlebia dahli (Rübsaamen) served as out group. Diclidophlebia is one of the two possible sister genera of Paurocephala (Burckhardt and Mifsud, in preparation). Successive weighting was applied when the first run resulted in several most parsimonious cladograms. The nelsen command was used to calculate consensus trees. Multistate characters were treated as unordered. For mapping the characters on the cladograms, the fast character optimization was used. Abbreviations: CI, consistency index; RI, retention index; $\mathrm{L}$, tree length.

\section{Character assessment}

A number of morphological characters, some of which were not previously used in the literature dealing with Paurocephala, are explained in the following section. In addition to qualitative characters, quantitative characters (tables $2 \mathrm{~A}-\mathrm{C}, 3$ ) were also used.

\section{Adult}

Head. Most species of Paurocephala show a moderately short head (figure 2A-C), but in some species such as $P$. brevicephala, the head is extremely short (figure 2D-F), with a high vertex length to width ratio. The length and density of setiferation on the vertex is of specific importance. Long setae on the vertex are present in $P$. chonchaiensis whereas the vertex of $P$. gossypii is covered by few inconspicuous setae. Rarely, tubercle-like structures are present on the fronto-lateral margins of the vertex such as in $P$. stephaniella. The labium length shows two extremes, very long (figure $21 \mathrm{G}$ ) as in $P$. psylloptera or short (figure $15 \mathrm{M}$ ) as in P. curvata. The antenna provides a number of diagnostic characters. In most species the antenna is longer than the head width, reaching the maximum in $P$. longiantennata. In other species it is extremely short, as long as or shorter than the head width, as in $P$. brevicephala. In most species the antenna is 10 -segmented but a small number of species such as $P$. brevicephala has 8 -segmented and $P$. kleinhofiae has 9-segmented 
Table 1. Checklist of Paurocephala spp. with information on distribution and host plants.

abutili sp. n. Distribution: Kenya. Host plant: Abutilon mauritianum (Jacq.) Medik. (Malvaceae).

ambigua sp. n. Distribution: Indonesia (Sulawesi). Host plant unknown.

artocarpae Braza and Calilung, 1981. Distribution: Philippines (Luzon), Malaysia (Sabah) and Indonesia (Sulawesi). Host plant: Artocarpus sp. (Moraceae).

bifasciata Kuwayama, 1931, stat. rev. Distribution: China (Hong Kong), Taiwan, Japan

(Kyushu, Shikoku, Honshu, and Ishigaki and Iriomote Islands) and Vietnam. Host plants:

Ficus erecta var. beecheyana (Hook. and Arn.) King, F. foveolata Pittier and Covellia hispida (L. f.) Miq. (Moraceae).

boehmeriae sp. n. Distribution: China (Hong Kong). Host plant. Boehmeria nivea (L.)

Gaudich. (Urticaceae).

boxi sp. n. Distribution: Ghana. Host plant unknown.

brendelli sp. n. Distribution: Malaysia (Malaysian Peninsula). Host plant unknown.

brevicephala (Crawford, 1917). Distribution: Philippines (Mindanao, Palawan) and Malaysia

(Sarawak). Host plant unknown.

calcarata sp. n. Distribution: Fiji Islands. Host plant unknown.

chonchaiensis Boselli, 1929 (=pumilae Li and Yang, 1987 syn. n., =zhejiangensis Yang and

Li, 1987 syn. n.). Distribution: China (including Hong Kong) and Japan. Host plant: Ficus pumila L. (Moraceae).

curvata sp. n. Distribution: Papua New Guinea and Indonesia (Sulawesi). Host plant unknown.

dayak sp. n. Distribution: Malaysia (Sabah and Sarawak), Brunei and Singapore. Host plants:

Trema tomentosa (Roxb.) Hara and Trema orientalis (L.) Blume (Ulmaceae).

debregeasiae Yang and Li, 1987, see sauteri Enderlein.

distincta sp. n. Distribution: India and Sri Lanka. Host plant unknown.

elegans sp. n. Distribution: Indonesia (Sulawesi). Host plant unknown.

gossypii Russell, 1943. Distribution: Zaire, Sudan and Malawi. Host plant: Gossypium sp.

(Malvaceae).

guangxiensis Yang and Li, 1987, see trematos Yang et al.

hollisi sp. $\mathrm{n}$. Distribution: Tanzania. Host plant unknown.

insolita sp. n. Distribution: Angola. Host plant: Psorospermum febrifugum Spach (Clusiaceae). javanica sp. n. Distribution: Indonesia (Java). Host plant: Ficus sp. (Moraceae).

kleinhofiae Uichanco, 1921, comb. rev. Distribution: Philippines (Luzon, Mindanao) and

Taiwan. Host plant: Kleinhovia hospita L. (Sterculiaceae).

lienhardi sp. n. Distribution: Malaysia (Sabah). Host plant unknown.

lii sp. n. Distribution: Indonesia (Sulawesi). Host plant unknown.

longiantennata sp. $\mathrm{n}$. Distribution: Papua New Guinea. Host plant unknown.

lucida sp. n. Distribution: Ethiopia. Host plant unknown.

macrochaetis sp. n. Distribution: Indonesia (Sulawesi). Host plant unknown.

maculipennis Uichanco, 1921. Distribution: Philippines including Palawan. Host plant: Ficus nota (Moraceae).

marginata sp. n. Distribution: Indonesia (Sulawesi). Host plant unknown.

martini sp. n. Distribution: Singapore. Host plant unknown.

mathuri sp. n. Distribution: India (Uttar Pradesh). Kydia calycina Roxb. (Malvaceae).

medleri sp. n. Distribution: Nigeria. Host plant unknown.

minuta Crawford, 1919. Distribution: Philippines (Luzon). Host plant unknown.

muiri sp. n. Distribution: Indonesia (Tanimbar Island). Host plant unknown.

muta sp. n. Distribution: India. Host plant unknown.

oceanica sp. n. Distribution: India (Andaman Islands). Host plant unknown.

palawanensis sp. $\mathrm{n}$. Distribution: Philippines (Palawan). Host plant: Trema orientalis (L.)

Blume (Ulmaceae).

papuana sp. n. Distribution: Papua New Guinea. Host plant unknown.

phalaki Mathur, 1975. Distribution: India (Bengal, Madras). Host plant unknown.

polaszeki sp. n. Distribution: Bangladesh. Host plant: Trema orientalis (L.) Blume (Ulmaceae).

psylloptera Crawford, 1913. Distribution: Philippines, Indonesia (Sumatra, Sulawesi) and

Malaysia (Sabah). Host plant: Ficus spp. (Moraceae). 
Table 1. (Continued).

pterospermi sp. n. Distribution: Philippines (Palawan) and Malaysia (Sabah). Host plant: Pterospermum sp. (Sterculiaceae).

pumilae Li and Yang, 1987, see chonchaiensis Boselli.

robusta sp. n. Distribution: Philippines (Palawan). Host plant unknown.

russellae Mathur, 1975. Distribution: India (Uttar Pradesh). Host plant: Kydia calycina Roxb.

(Malvaceae).

sauteri Enderlein, 1914 (=debregeasiae Yang and Li, 1987 syn. n.). Distribution: China and

Taiwan. Host plant: Morus alba L. (Moraceae).

setifera (Crawford, 1919). Distribution: Philippines (Luzon). Host plant: Trema orientalis (L.)

Blume (Ulmaceae).

similis $\mathrm{sp}$. n. Distribution: Indonesia (Sulawesi). Host plant unknown.

sinuata sp. n. Distribution: Ghana and Nigeria. Host plant unknown.

stephaniella sp. n. Distribution: Indonesia (Sulawesi). Host plant unknown.

stigmaticalis sp. n. Distribution: Malaysia (Sabah). Host plant unknown.

sulawesiana sp. n. Distribution: Indonesia (Sulawesi). Host plant unknown.

tremae Yang and Li, 1987, see trematos Yang et al.

trematos Yang et al., 1986 (= guangxiensis Yang and Li, 1987 syn. n., =tremae Yang and Li,

1987). Distribution: Taiwan, Japan, Thailand, China and Malaysia. Host plant: Trema orientalis (L.) Blume (Ulmaceae).

urenae Russell, 1946. Distribution: Zaire. Host plant: Urena lobata L. (Malvaceae).

wilderi Crawford, 1927. Distribution: American Samoa. Host plant unknown.

zhejiangensis Yang and Li, 1987, see chonchaiensis Boselli.

antenna. In 10-segmented antenna, a single rhinarium is present subapically on segments 4, 6, 8 and 9; in 9-segmented antenna on segments 3, 5, 7 and 8; in 8 -segmented antenna on segments 3, 4, 6 and 7. The presence or absence of a long basal seta on distal antennal segments offers a good character for species discrimination. Some species, such as $P$. psylloptera, have such a seta on segment 9 , others such as $P$. brevicephala, on segments 7 and 8 . The length of the terminal setae is in general much shorter than the antennal flagellum, but in a few species such as $P$. brevicephala and $P$. elegans it is as long as or longer than the flagellum. Antennal rhinaria often bear a single transparent seta, which is relatively long as in $P$. muiri. The Afrotropical species mostly lack these setae, but in species such as $P$. brevicephala and $P$. kleinhofiae a bifid seta (figure $2 \mathrm{~J}$ ) is present reaching the maximum expression in $P$. martini.

Hind legs. The meracanthus is always present; short and apically blunt (figure $7 \mathbf{J}$ ) as in $P$. gossypii, long curved ventrally and subacute apically (figure $28 \mathrm{~N}$ ) as in $P$. setifera and very long, straight ventrally (figure 14G) as in $P$. calcarata. The majority of species such as $P$. psylloptera and $P$. chonchaiensis have a relatively long metatibia, in $P$. brevicephala it is short and as stout as long or shorter than the metafemur. The Afrotropical species are intermediate in this character. Apical metatibial spurs are usually not strongly sclerotized nor black, a character shared by some Neotropical Diclidophlebia spp. The metatibia usually bears similar spurs laterally, forming two rows in P. psylloptera, one row in P. brevicephala and reduced to two or three stout setoe in $P$. wilderi. The Afrotropical species and two Asian species lack these lateral stout setae.

Forewing. The venation and setiferation of the forewing provide diagnostic characters for species identification. The length and density of setiferation on forewing veins is in general of specific importance. The length and ratios of several veins and connections therein are also useful for species determination. The pterostigma can 
Table 2A. Measurements of adult Paurocephala species ( $N=$ number of measured specimens).

\begin{tabular}{|c|c|c|c|c|c|c|c|c|}
\hline Species & $N$ & HW & WL & $\mathrm{MC}$ & MP & PL & $\mathrm{DL}$ & FP \\
\hline abutili & $2{ }^{\star} 2$ 우 & 0.49 & $1.59-1.73$ & $0.07-0.08$ & $0.18-0.19$ & $0.18-0.19$ & 0.11 & $0.54-0.57$ \\
\hline ambigua & $2 \delta 20$ & $0.66-0.75$ & $1.61-2.01$ & $0.07-0.08$ & $0.14-0.15$ & $0.23-0.24$ & $0.21-0.22$ & $0.61-0.66$ \\
\hline artocarpae & 303 우 & $0.62-0.70$ & $1.74-2.10$ & $0.11-0.12$ & $0.23-0.25$ & $0.28-0.30$ & $0.23-0.24$ & $0.77-0.84$ \\
\hline bifasciata & 40 4 우 & $0.58-0.63$ & $1.80-2.02$ & $0.09-0.14$ & $0.20-0.21$ & $0.15-0.18$ & $0.14-0.16$ & $0.67-0.72$ \\
\hline boehmeriae & 202 우 & $0.57-0.60$ & $1.71-1.95$ & $0.10-0.11$ & 0.23 & 0.22 & $0.17-0.18$ & $0.69-0.71$ \\
\hline boxi & $10^{\pi} 1$ 우 & $0.50-0.53$ & 1.30 & $0.04-0.05$ & 0.20 & 0.19 & 0.15 & 0.53 \\
\hline brendelli & $2{ }^{\star} 1$ 우 & $0.56-0.59$ & $1.44-1.63$ & $0.05-0.08$ & $0.14-0.16$ & $0.11-0.12$ & 0.13 & 0.54 \\
\hline brevicephala & $1 亏 1$ 우 & $0.67-0.72$ & $1.54-1.85$ & $0.07-0.08$ & 0.13 & 0.14 & 0.12 & 0.52 \\
\hline calcarata & $30^{\star} 19$ & $0.49-0.56$ & $1.51-1.68$ & $0.18-0.19$ & $0.15-0.16$ & $0.18-0.19$ & 0.14 & 0.63 \\
\hline chonchaiensis & 4ठ 4 우 & $0.52-0.62$ & $1.44-1.76$ & $0.09-0.11$ & $0.17-0.19$ & $0.14-0.17$ & $0.13-0.15$ & $0.54-0.60$ \\
\hline curvata & $2 \widehat{O} 1$ 우 & $0.60-0.62$ & $1.59-1.76$ & $0.10-0.11$ & $0.19-0.20$ & $0.20-0.21$ & 0.19 & 0.58 \\
\hline dayak & $60^{\wedge} 6$ 우 & $0.56-0.65$ & $1.56-2.05$ & $0.08-0.11$ & $0.22-0.25$ & $0.24-0.30$ & $0.18-0.21$ & $0.71-0.91$ \\
\hline distincta & 101 우 & $0.73-0.75$ & $2.12-2.24$ & $0.17-0.18$ & 0.25 & 0.23 & 0.16 & 0.75 \\
\hline elegans & 101 1운 & 0.46 & $1.07-1.27$ & $0.05-0.06$ & 0.17 & 0.11 & 0.12 & 0.56 \\
\hline gossypii & $2{ }^{*} 2$ 우 & $0.48-0.51$ & $1.29-1.49$ & $0.06-0.07$ & $0.15-0.16$ & $0.17-0.18$ & $0.11-0.12$ & $0.49-0.55$ \\
\hline hollisi & $4 \widehat{O} 1$ 우 & $0.50-0.58$ & $0.88-1.51$ & $0.05-0.07$ & $0.15-0.16$ & $0.18-0.20$ & $0.16-0.18$ & 0.59 \\
\hline insolita & 202 우 & $0.54-0.57$ & $1.49-1.61$ & 0.08 & 0.19 & 0.20 & $0.16-0.17$ & $0.60-0.61$ \\
\hline javanica & $3 \sigma^{1} 3 q$ & $0.64-0.74$ & $1.78-2.15$ & $0.12-0.13$ & $0.21-0.24$ & $0.25-0.27$ & $0.19-0.23$ & $0.72-0.75$ \\
\hline klienhofiae & 1011 우 & $0.47-0.51$ & $1.05-1.29$ & $0.05-0.06$ & 0.15 & 0.11 & 0.10 & 0.48 \\
\hline lateralis & $1 \widehat{\sigma}$ & 0.43 & 1.10 & 0.04 & 0.11 & 0.09 & 0.07 & - \\
\hline lienhardi & $1 \sigma^{\star} 1$ 우 & $0.53-0.56$ & $1.71-1.95$ & 0.08 & 0.16 & 0.12 & 0.10 & 0.52 \\
\hline lii & 101 우 & $0.51-0.54$ & $1.34-1.41$ & 0.06 & 0.15 & 0.11 & 0.16 & 0.51 \\
\hline longiantennata & $2 \widehat{0} 2$ 우 & $0.75-0.84$ & $2.59-3.15$ & $0.12-0.14$ & $0.35-0.36$ & $0.29-0.30$ & - & $1.15-1.16$ \\
\hline lucida & 19 & 0.59 & 2.20 & 0.08 & - & - & - & 0.71 \\
\hline macrochaetis & 1011 웅 & $0.57-0.58$ & $1.50-1.62$ & $0.08-0.09$ & 0.20 & 0.18 & 0.16 & 0.65 \\
\hline maculipennis & $5 \lesssim 3 q$ & $0.65-0.75$ & $1.98-2.32$ & $0.09-0.10$ & $0.25-0.29$ & 0.25 & $0.19-0.21$ & $0.80-0.90$ \\
\hline marginata & $2 \sigma 20$ & $0.62-0.66$ & $1.95-2.15$ & $0.09-0.10$ & 0.24 & 0.28 & $0.20-0.21$ & $0.81-0.83$ \\
\hline martini & $1 \delta 1$ 우 & $0.61-0.67$ & $1.68-1.83$ & $0.07-0.08$ & 0.17 & 0.14 & 0.14 & 0.53 \\
\hline mathuri & 1011 우 & $0.55-0.58$ & $1.46-1.63$ & $0.05-0.06$ & 0.14 & 0.14 & - & 0.53 \\
\hline medleri & $1 \delta 1$ 东 & $0.49-0.51$ & $1.32-1.37$ & $0.04-0.05$ & 0.21 & 0.19 & 0.16 & 0.58 \\
\hline muiri & 1011 和 & $0.59-0.60$ & $1.49-1.56$ & $0.12-0.13$ & 0.20 & 0.20 & 0.16 & 0.72 \\
\hline muta & 40140 & $0.55-0.59$ & $1.51-1.81$ & $0.09-0.10$ & $0.22-0.24$ & $0.24-0.26$ & $0.19-0.20$ & $0.80-0.83$ \\
\hline oceanica & $1 \widehat{0} 1$ 우 & $0.59-0.63$ & $1.68-1.78$ & $0.08-0.09$ & 0.24 & 0.25 & 0.19 & 0.79 \\
\hline palawanensis & $1 \% 2 q$ & $0.58-0.62$ & $1.49-1.80$ & $0.08-0.09$ & 0.23 & 0.24 & 0.19 & $0.78-0.90$ \\
\hline papuana & $2 \lesssim 20$ & $0.58-0.64$ & $1.74-1.90$ & $0.13-0.15$ & $0.21-0.22$ & $0.19-0.20$ & 0.17 & $0.81-0.85$ \\
\hline polaszeki & 202 우 & $0.56-0.57$ & $1.41-1.56$ & 0.09 & $0.21-0.22$ & $0.21-0.22$ & $0.15-0.17$ & 0.65 \\
\hline psylloptera & 505 우 & $0.58-0.67$ & $1.63-2.07$ & $0.14-0.17$ & $0.20-0.22$ & $0.21-0.23$ & $0.17-0.18$ & $0.76-0.89$ \\
\hline pterospermi & $4 \lesssim 4+$ & $0.61-0.71$ & $1.59-1.83$ & 0.08 & 0.14 & $0.19-0.20$ & $0.18-0.20$ & $0.55-0.58$ \\
\hline robusta & $2 \widehat{\delta} 2$ 우 & $0.64-0.66$ & $0.68-1.49$ & $0.06-0.07$ & $0.14-0.17$ & 0.15 & $0.12-0.16$ & $0.54-0.55$ \\
\hline sauteri & $3 \widehat{\delta} 2$ 우 & $0.58-0.66$ & $1.71-2.10$ & 0.09 & $0.21-0.23$ & $0.21-0.23$ & $0.16-0.17$ & $0.72-0.73$ \\
\hline setifera & $2 \widehat{\delta} 2$ 우 & $0.58-0.62$ & $1.71-1.93$ & $0.08-0.10$ & $0.25-0.28$ & $0.28-0.30$ & $0.21-0.22$ & $0.88-0.89$ \\
\hline similis & $10 x 2$ 우 & $0.60-0.65$ & $1.68-1.95$ & $0.12-0.13$ & 0.20 & 0.22 & 0.20 & $0.83-0.86$ \\
\hline sinuata & $3 \delta 3 q$ & $0.47-0.51$ & $1.27-1.49$ & $0.07-0.08$ & $0.15-0.16$ & 0.14 & 0.14 & $0.55-0.56$ \\
\hline stigmaticalis & 1 우 & 0.52 & 1.78 & 0.08 & - & - & - & 0.80 \\
\hline sulawesiana & 2019 & $0.65-0.71$ & $1.54-1.88$ & 0.08 & $0.14-0.15$ & 0.13 & $0.12-0.14$ & 0.55 \\
\hline trematos & $1 \delta 1$ 웅 & $0.57-0.62$ & $1.66-1.88$ & $0.09-0.10$ & 0.22 & $0.23-0.24$ & 0.18 & 0.72 \\
\hline urenae & $1 \widehat{\delta} 1$ 우 & $0.45-0.47$ & $1.44-1.56$ & $0.07-0.08$ & 0.15 & 0.17 & 0.14 & 0.54 \\
\hline wilderi & $1 \delta 1 \%$ & $0.53-0.55$ & $1.56-1.71$ & 0.12 & 0.16 & 0.21 & 0.15 & 0.56 \\
\hline
\end{tabular}

be either as transparent as the rest of the forewing or completely brown as in $P$. marginata. The shape and length of pterostigma in relation to the length of the Rs vein is used to separate species groups. The apices of veins along the outer wing margin sometimes bear a dark dot as in P. pterospermi, or the veins are darkened with a marginal diffused pattern as in $P$. marginata. In general, most Paurocephala spp. have no distinct wing pattern; notable exceptions are $P$. chonchaiensis, $P$. phalaki and $P$. bifasciata with two brown bands, one in the middle stretching from the anal to costal break and one apically (figure 12A, B). In $P$. stephaniella an extensive 
Table 2B. Ratios of adult Paurocephala species.

\begin{tabular}{|c|c|c|c|c|c|c|c|c|}
\hline Species & $\mathrm{AL} / \mathrm{HW}$ & $\mathrm{AL} / \mathrm{F} 1$ & LB/HW & WL/HW & $\mathrm{WL} / \mathrm{V}$ & $\mathrm{PT} / \mathrm{WL}$ & RL/WL & $\mathrm{RL} / \mathrm{RC}$ \\
\hline butili & & & & & & & & \\
\hline & & & & & & & & \\
\hline & & & & & & & & \\
\hline & & & $22-0.25$ & $2.98-3.30$ & & & & \\
\hline & & & & & & & & \\
\hline & & & 0.16 & 2.59 & 2.16 & 0.31 & & 1. \\
\hline$l l i$ & & & & & & & & \\
\hline ohala & & & & & & & & \\
\hline & & & & & & & & \\
\hline ensis & & & & & & & & \\
\hline & & & & & & & & \\
\hline & & & & & & & & \\
\hline & & & & & & & & \\
\hline & & & & & & & & \\
\hline & & & & & & & & \\
\hline & & & & & & & & \\
\hline & & & & & & & & \\
\hline & & & & & & & & \\
\hline & & & & & & & & 1.34 \\
\hline & & & & & & & & \\
\hline & & & & & & & & \\
\hline & & $3.91-$ & & & & & & \\
\hline tennata & & & & & & & & \\
\hline & & & & & & & & \\
\hline & & & & & & & & \\
\hline & & & & & & & & \\
\hline & & & & & & & & \\
\hline & & & & & & & & \\
\hline & & & & & & & & \\
\hline & & & & & & & & \\
\hline & & & & & & & & \\
\hline & & & & & & & & \\
\hline & & & & & & & & \\
\hline & & & & & & & & \\
\hline & & & & & & & & \\
\hline & & & & & & & & \\
\hline & & & & & & & & \\
\hline & & & & & & & & \\
\hline & & & & & & & & \\
\hline & & & & & & & & \\
\hline & & & & & & & & \\
\hline & & & & & & & & \\
\hline & $1.08-1.23$ & $5.08-6$ & $0.17-0.20$ & $2.59-2.98$ & $2.30-2.44$ & $0.17-0.21$ & $0.46-0.49$ & $1.22-1.33$ \\
\hline & & & & & & & & \\
\hline & & & & & & 0.25 & 0.47 & 1.36 \\
\hline & & & & & & & & \\
\hline & & & & & & & & $91.27-1.4$ \\
\hline wilderi & & & $0.10-0$ & $2.95-3.12$ & 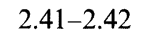 & $0.27-0.28$ & $0.51-0.53$ & 1.33 \\
\hline
\end{tabular}

brown pattern is present apically. Surface spinules on the wing membrane can be completely absent as in $P$. elegans, present in cells within the distal half of the wing as in $P$. psylloptera or cover all cells as in P. robusta. Surface spinules tend to form more extensive fields in females than in males. The density of surface spinules can be dense as in $P$. chonchaiensis or sparse as in P. bifasciata. An additional character is the absence, as in P. elegans, or presence, as in P. psylloptera, of radular spinules.

Thorax. All Paurocephala species possess a horn on metascutellum (figure $2 \mathrm{G}-\mathrm{H}$ ). It is shallow in species such as $P$. urenae and $P$. wilderi, basally wide and short as 
Table 2C. Ratios of adult Paurocephala species.

\begin{tabular}{|c|c|c|c|c|c|c|c|c|}
\hline Species & $a / b$ & $\mathrm{a} / \mathrm{c}$ & $\mathrm{d} / \mathrm{e}$ & ML/HW & $\mathrm{MP} / \mathrm{HW}$ & $\mathrm{FP} / \mathrm{HW}$ & $\mathrm{FP} / \mathrm{SL}$ & $\mathrm{FP} / \mathrm{CL}$ \\
\hline abutili & $1.40-1.46$ & $1.02-1.27$ & $3.93-4.24$ & $1.25-1.37$ & $0.37-0.38$ & $1.10-1.15$ & $2.07-2.19$ & 3.00 \\
\hline ambigua & $1.30-1.42$ & $1.11-1.28$ & $3.00-3.59$ & $0.74-0.83$ & $0.21-0.23$ & $0.82-0.90$ & $2.69-2.71$ & $2.59-2.83$ \\
\hline artocarpae & $1.38-1.49$ & $1.15-1.23$ & $3.40-3.92$ & $1.37-1.46$ & $0.36-0.40$ & $1.12-1.27$ & $1.91-1.98$ & $3.56-3.90$ \\
\hline bifasciata & $1.31-1.44$ & $1.18-1.35$ & $3.06-3.87$ & $1.24-1.28$ & $0.33-0.35$ & $1.09-1.17$ & $2.11-2.29$ & $3.23-3.57$ \\
\hline boehmeriae & $1.54-1.76$ & $0.98-1.11$ & $4.29-5.27$ & $1.33-1.43$ & $0.39-0.40$ & $1.17-1.22$ & 1.74 & $3.32-3.75$ \\
\hline boxi & 1.17 & 1.17 & 2.78 & $0.89-0.94$ & 0.40 & 1.00 & 2.33 & 4.00 \\
\hline brendelli & $1.36-1.43$ & $1.67-1.79$ & $2.94-3.21$ & $0.76-0.79$ & $0.25-0.29$ & 0.90 & 2.19 & 2.71 \\
\hline brevicephala & $1.43-1.75$ & $1.27-1.31$ & $3.82-4.26$ & $0.66-0.72$ & 0.20 & 0.72 & 3.06 & 3.67 \\
\hline calcarata & $1.35-1.52$ & $1.09-1.25$ & $3.83-4.17$ & $1.36-1.48$ & $0.29-0.31$ & 1.14 & 1.86 & 3.53 \\
\hline chonchaiensis & $1.23-1.34$ & $1.28-1.61$ & $2.85-3.19$ & $0.98-1.14$ & $0.29-0.34$ & $0.97-1.04$ & $2.42-2.56$ & $3.00-3.20$ \\
\hline curvata & $1.38-1.39$ & $1.11-1.29$ & $3.19-3.43$ & $1.08-1.11$ & $0.31-0.32$ & 0.92 & 2.44 & 3.81 \\
\hline dayak & $1.35-1.54$ & $1.06-1.27$ & $3.52-4.23$ & $1.20-1.42$ & $0.37-0.42$ & $1.23-1.39$ & $1.88-2.21$ & $3.26-3.48$ \\
\hline distincta & $1.32-1.43$ & $1.25-1.61$ & $4.19-5.13$ & $1.44-1.50$ & 0.34 & 0.99 & 2.55 & 3.43 \\
\hline elegans & $1.28-1.35$ & $1.44-1.52$ & $2.71-2.91$ & 0.90 & 0.36 & - & 2.27 & 3.69 \\
\hline gossypii & $1.32-1.44$ & $1.30-1.42$ & $3.82-4.56$ & $0.92-1.00$ & $0.30-0.32$ & $1.02-1.09$ & $2.08-2.23$ & $3.22-3.47$ \\
\hline hollisi & $1.52-1.80$ & $0.97-1.02$ & $2.75-3.00$ & $0.98-1.02$ & $0.29-0.31$ & 1.02 & 3.00 & 2.86 \\
\hline insolita & $1.23-1.35$ & $1.30-1.46$ & $2.39-2.63$ & $0.87-0.95$ & $0.33-0.35$ & $1.07-1.12$ & $2.00-2.17$ & $3.25-3.37$ \\
\hline javanica & $1.27-1.39$ & $1.14-1.27$ & $2.95-3.38$ & $1.28-1.37$ & $0.32-0.34$ & $0.97-1.05$ & $1.90-2.00$ & $2.96-3.08$ \\
\hline klienhofiae & $1.29-1.31$ & $1.17-1.37$ & $3.04-3.14$ & $0.81-0.83$ & 0.32 & 0.94 & 2.55 & 2.83 \\
\hline lateralis & 1.35 & 1.64 & 3.40 & 0.70 & 0.26 & - & - & - \\
\hline lienhardi & $1.62-1.63$ & $1.00-1.03$ & $4.06-4.33$ & $0.93-0.95$ & 0.30 & 0.93 & 2.29 & 2.39 \\
\hline lii & $1.45-1.48$ & $1.35-1.38$ & $2.96-3.58$ & $0.84-0.89$ & 0.30 & 0.95 & 2.25 & 3.38 \\
\hline longiantennata & $1.62-1.98$ & $1.05-1.20$ & $3.48-3.80$ & $1.51-1.61$ & $0.45-0.46$ & $1.38-1.39$ & $2.05-2.07$ & $3.97-4.07$ \\
\hline lucida & 2.04 & 1.12 & 3.76 & 1.11 & - & 1.19 & 2.34 & 3.95 \\
\hline macrochaetis & $1.47-1.52$ & $1.06-1.08$ & $4.57-4.80$ & $1.45-1.47$ & 0.35 & 1.11 & 1.92 & 3.63 \\
\hline maculipennis & $1.63-1.86$ & $1.02-1.13$ & $3.44-3.82$ & $1.33-1.59$ & $0.36-0.42$ & $1.19-1.23$ & $2.07-2.11$ & $3.54-3.96$ \\
\hline marginata & $1.52-1.77$ & $1.04-1.10$ & $4.00-4.20$ & $1.45-1.58$ & 0.38 & $1.23-1.31$ & 2.10 & $3.74-4.00$ \\
\hline artini & $1.44-1.46$ & $1.21-1.26$ & $3.18-3.33$ & $0.77-0.85$ & 0.28 & 0.79 & 2.80 & 3.11 \\
\hline athuri & $1.67-1.78$ & $1.14-1.25$ & $3.29-3.33$ & $0.94-0.97$ & 0.25 & 0.90 & 2.67 & 3.11 \\
\hline edleri & $1.43-1.50$ & $1.11-1.15$ & $2.92-3.00$ & $1.04-1.06$ & 0.42 & 1.13 & 2.26 & 4.07 \\
\hline muiri & $1.48-1.54$ & $1.03-1.14$ & $4.08-4.24$ & $1.22-1.27$ & 0.34 & 1.19 & 2.08 & 3.23 \\
\hline muta & $1.34-1.58$ & $1.00-1.09$ & $4.08-4.58$ & $1.40-1.54$ & $0.38-0.41$ & $1.34-1.44$ & $2.05-2.26$ & $3.58-3.83$ \\
\hline oceanica & $1.45-1.50$ & $1.13-1.14$ & $3.67-3.73$ & $1.34-1.35$ & 0.40 & 1.25 & 2.33 & 3.65 \\
\hline palawanensis & $1.32-1.52$ & $1.23-1.39$ & $3.73-4.17$ & $1.16-1.38$ & 0.39 & $1.32-1.44$ & $1.90-1.93$ & $3.46-3.80$ \\
\hline papuana & $1.48-1.67$ & $1.00-1.15$ & $4.22-4.88$ & $1.46-1.56$ & $0.35-0.37$ & $1.26-1.38$ & 1.91 & $4.09-4.10$ \\
\hline polaszeki & $1.32-1.48$ & $1.09-1.18$ & $3.26-3.77$ & $0.97-1.00$ & $0.37-0.39$ & 1.15 & 2.30 & 3.14 \\
\hline psylloptera & $1.39-1.61$ & $0.98-1.19$ & $3.84-4.58$ & $1.40-1.69$ & $0.31-0.36$ & $1.21-1.34$ & $1.93-2.09$ & $3.48-3.75$ \\
\hline pterospermi & $1.29-1.50$ & $1.07-1.67$ & $3.47-3.88$ & $0.70-0.77$ & $0.21-0.23$ & $0.78-0.86$ & & $2.52-2.95$ \\
\hline robusta & $1.46-1.53$ & $1.17-1.31$ & $3.22-3.63$ & $0.68-0.76$ & $0.22-0.26$ & $0.81-0.83$ & $3.17-3.63$ & $2.85-2.90$ \\
\hline sauteri & $1.60-1.68$ & $1.06-1.19$ & $4.88-5.33$ & $1.18-1.36$ & $0.35-0.37$ & $1.10-1.15$ & $2.05-2.20$ & $3.80-3.85$ \\
\hline setifera & $1.47-1.54$ & $1.11-1.29$ & $3.28-4.20$ & $1.40-1.63$ & $0.44-0.48$ & $1.42-1.48$ & & $3.72-3.92$ \\
\hline similis & $1.36-1.41$ & $1.15-1.19$ & $3.88-4.08$ & $1.39-1.50$ & 0.33 & $1.28-1.34$ & $1.82-1.83$ & $4.00-4.14$ \\
\hline sinuata & $1.42-1.54$ & $1.13-1.21$ & $3.13-3.54$ & $0.90-1.00$ & $0.30-0.34$ & 1.09 & $2.68-3.05$ & $2.81-2.90$ \\
\hline stigmaticalis & 1.83 & 1.00 & 5.79 & 1.44 & - & 1.55 & 2.13 & 3.27 \\
\hline sulawesiana & $1.47-1.52$ & $1.38-1.67$ & $3.50-4.36$ & $0.68-0.72$ & $0.21-0.23$ & 0.77 & 2.90 & 2.90 \\
\hline trematos & $1.41-1.52$ & $1.07-1.14$ & $3.85-4.20$ & $1.10-1.17$ & $0.35-0.38$ & 1.16 & 1.95 & 3.17 \\
\hline urenae & $1.38-1.45$ & $1.26-1.29$ & $3.33-4.00$ & $1.06-1.10$ & 0.33 & 1.14 & 1.90 & 3.00 \\
\hline wilderi & $1.71-1.77$ & $1.05-1.08$ & $4.80-5.00$ & 1.16 & 0.30 & 1.02 & 2.81 & 2.95 \\
\hline
\end{tabular}

in $P$. brevicephala and distinctly long as in $P$. psylloptera, $P$. chonchaiensis and $P$. elegans. The length and density of setiferation on the dorsum of thorax is of specific importance. Long setae are present in $P$. setifera, whereas $P$. gossypii has short setae.

Abdomen. On the sides of the first visible abdominal tergite, a patch of small inconspicuous to relatively long spines represents an autapomorphy of the Paurocephalinae. In some species, such as $P$. setifera and $P$. maculipennis, it forms a stalked process which is forward directed. This structure is considerably larger in males than in females (figure 4A, B). A horn-like structure is present on each tergite 


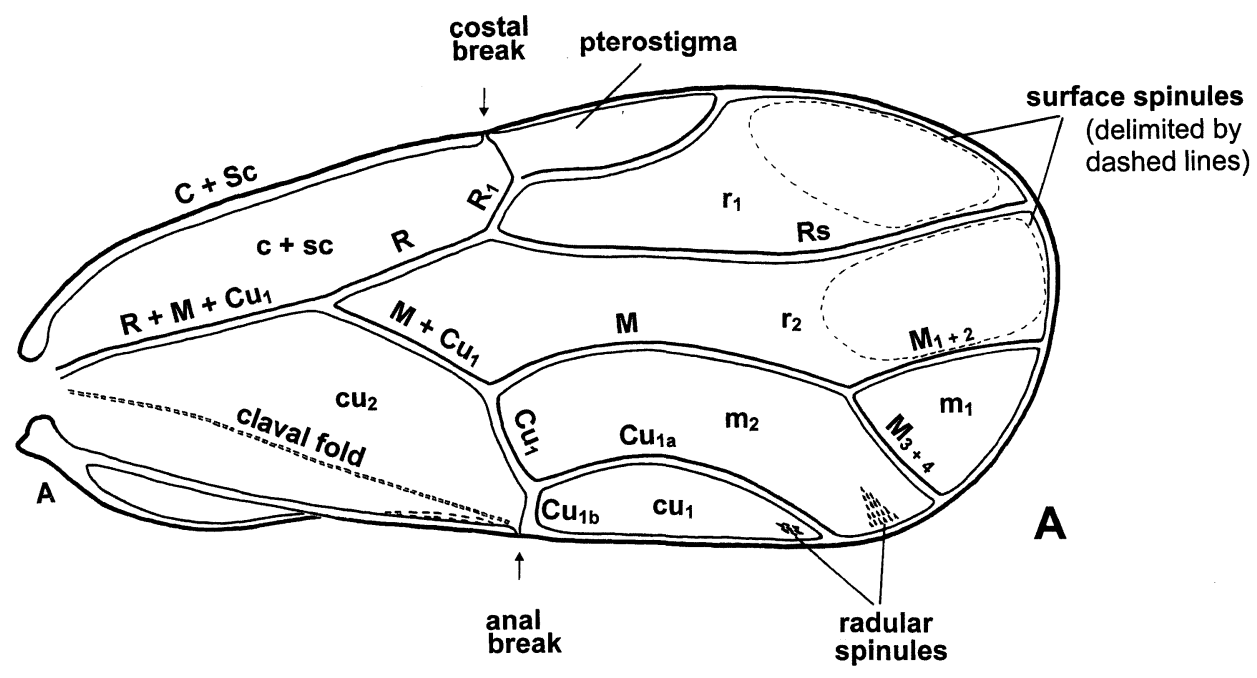

WL

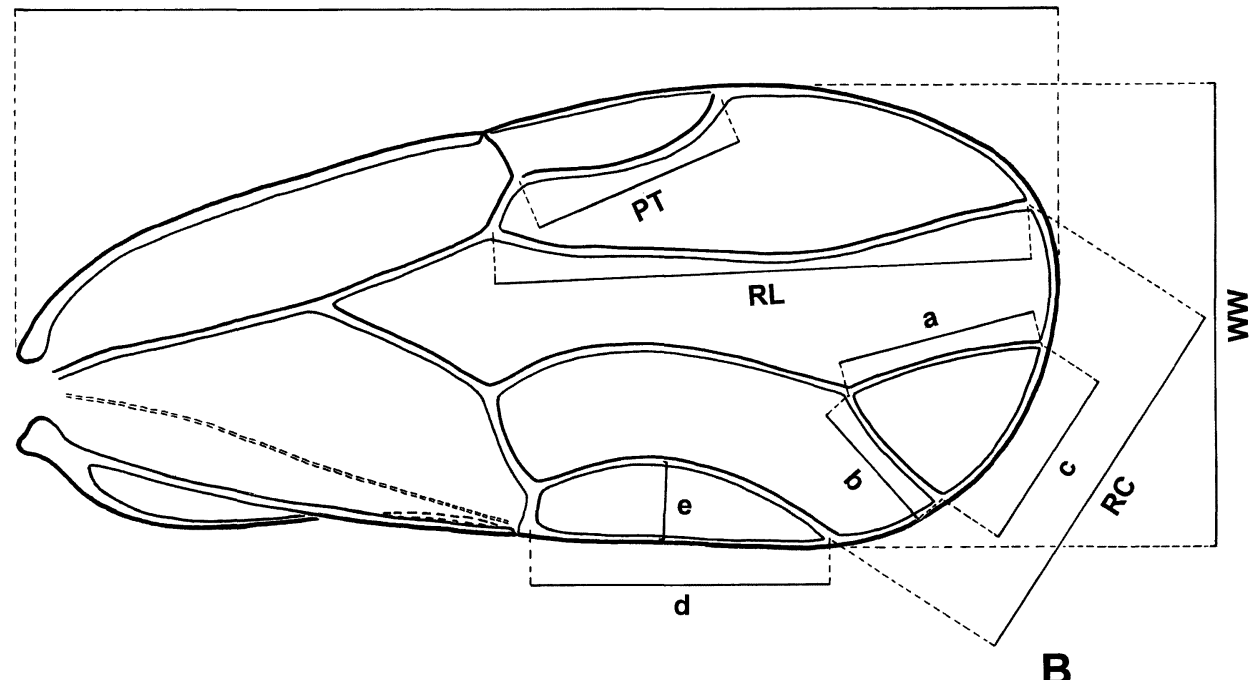

Fig. 1. Forewing: (A) morphological terminology; (B) lines indicating measurements when quantifying length of veins and connections.

in a few species as $P$. russellae. On the tergites short simple setae are generally present laterally whereas in $P$. lucida stout setae are present on the first four visible tergites (figure 5I).

Genitalia. As in other psylloids, genitalia offer a multitude of good diagnostic and phylogenetically informative characters. The male proctiger is simple and tubular (figures $14 \mathrm{~A}-\mathrm{F}, 30 \mathrm{~A}-\mathrm{C}$ ), as in $P$. brevicephala, and $P$. wilderi, but in the majority of cases it bears distinctly subdivided plates posteriorly (figures 7A-C, 23). Setae on the inner surface of the paramere may be simple as in P. brevicephala, stout and short (figure 15D) as in P. wilderi, and P. psylloptera, stout and long (figure 15B) as in $P$. artocarpae or massive and peg-like (figure 7D, E) as in P. gossypii. The number 

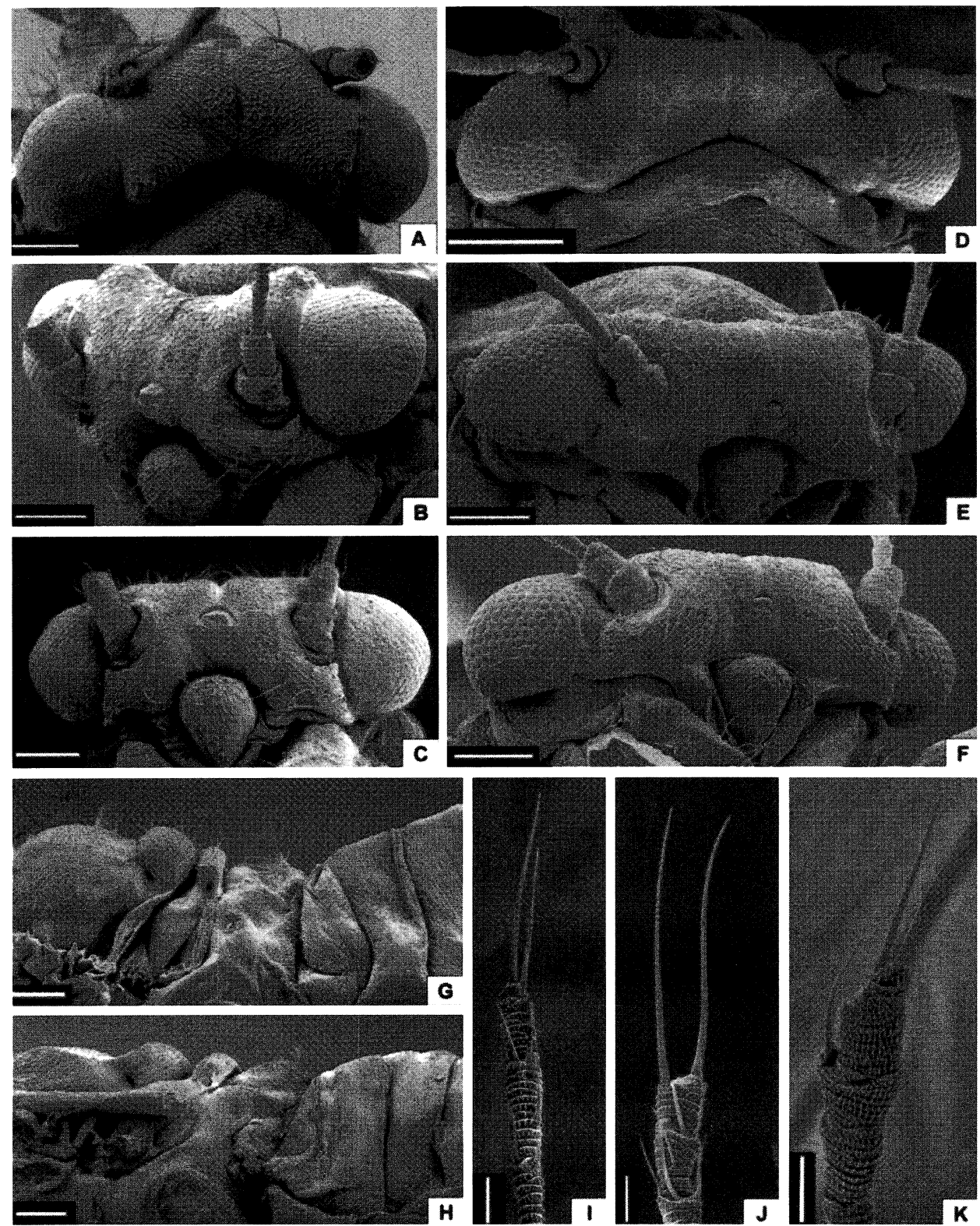

FIG. 2. Paurocephala spp.: (A-C, G, I) P. psylloptera; (D-F) P. sulawesiana; (H) P. cf. brevicephala; (J) P. martini; (K) P. hollisi. (A, D) Head, dorsal view; (B, E) head, oblique frontal view; $(\mathrm{C}, \mathrm{F})$ head, oblique ventral view; $(\mathrm{G}, \mathrm{H})$ metascutellar horn, lateral view; (I-K) distal antennal segments. Scale bar: A-H, $0.1 \mathrm{~mm}$; I-K, $0.03 \mathrm{~mm}$.

and position of these setae are important for species determination. The apex of the proximal segment of the aedeagus is often inflated and longer postero-ventrally than antero-dorsally. The apical part of distal portion is usually well differentiated from the stem (figure 10C, D), as in P. kleinhofiae; it is less differentiated (figure 32I-N) in species such as $P$. brevicephala. In most cases, the female genitalia are cuneate and relatively long (figure $3 \mathrm{~A}$ ) as in $P$. setifera, but in some species they are short 

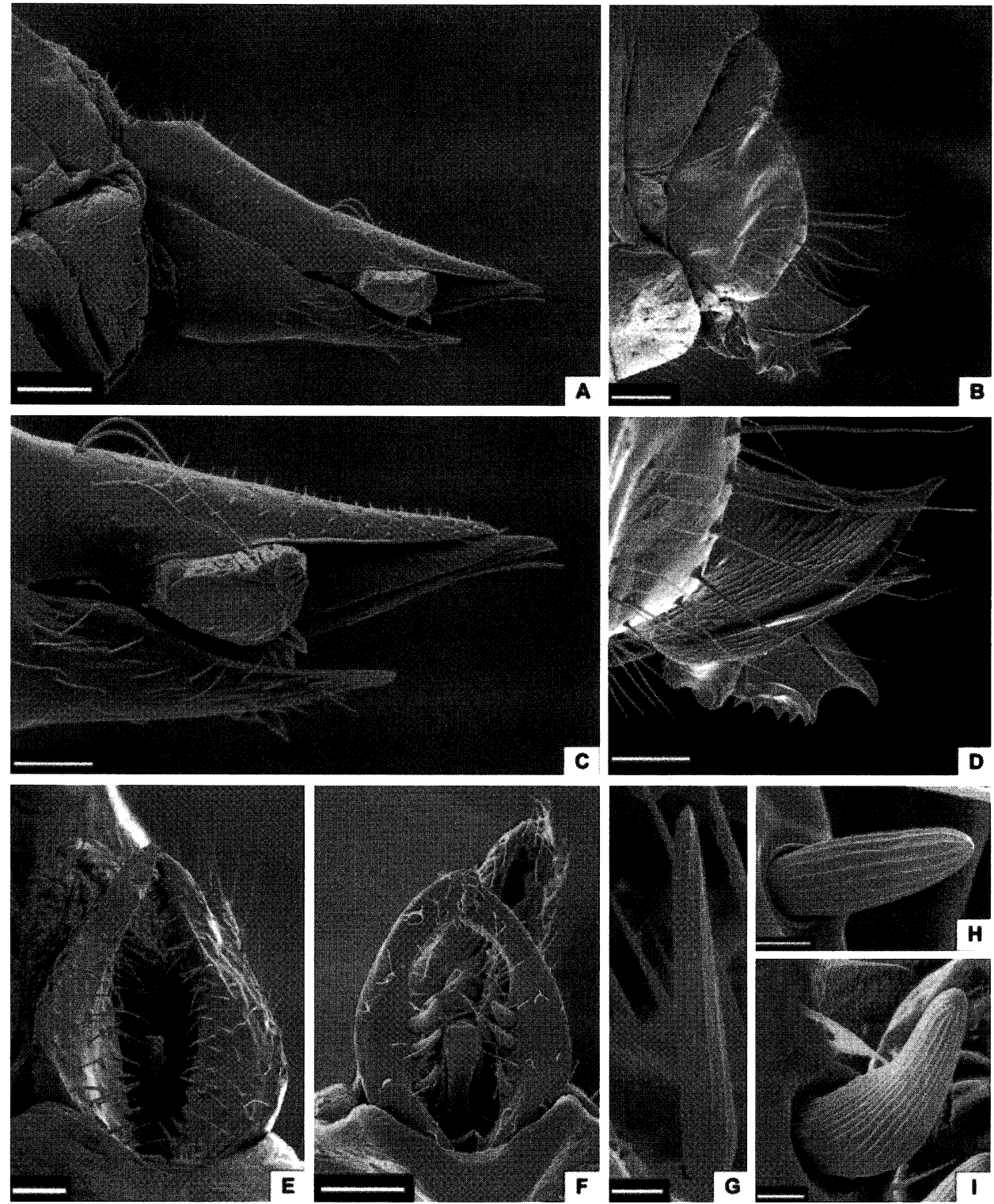

FIG. 3. Paurocephala spp.: (A, B, E, H) P. psylloptera; (C, D) P. martini; (F, I) P. gossypii; (G) P. artocarpae. (A, C) Female genitalia, lateral view; (B, D) apex of female genitalia with lateral valvula; (E, F) paramere, posterior view; $(\mathrm{G}-\mathrm{I})$ stout and peg-like seta on inner surface of paramere. Scale bar: A, C, $0.1 \mathrm{~mm} ; \mathrm{B}, \mathrm{D}, \mathrm{F}, 0.05 \mathrm{~mm} ; \mathrm{E}, 0.03 \mathrm{~mm}$; $\mathrm{G}, \mathrm{I}, 0.005 \mathrm{~mm} ; \mathrm{H}, 0.003 \mathrm{~mm}$.

and upturned apically (figure 3B) as in P. brevicephala. The circumanal ring can be oval (figure 6C) or cruciform (figure 6D) and the number of pore rows can be two or reduced to one (figure $16 \mathrm{C}, \mathrm{D}, \mathrm{F}$ ) as in $P$. wilderi. The lateral valvulae are usually membranous (figure $3 \mathrm{C}$ ) but in a few species, such as $P$. brevicephala and $P$. mathuri, they are highly sclerotized with ventral serrations (figure 3D).

Fifth instar larva. The antenna is often distinctly 3 -segmented as in P. urenae 

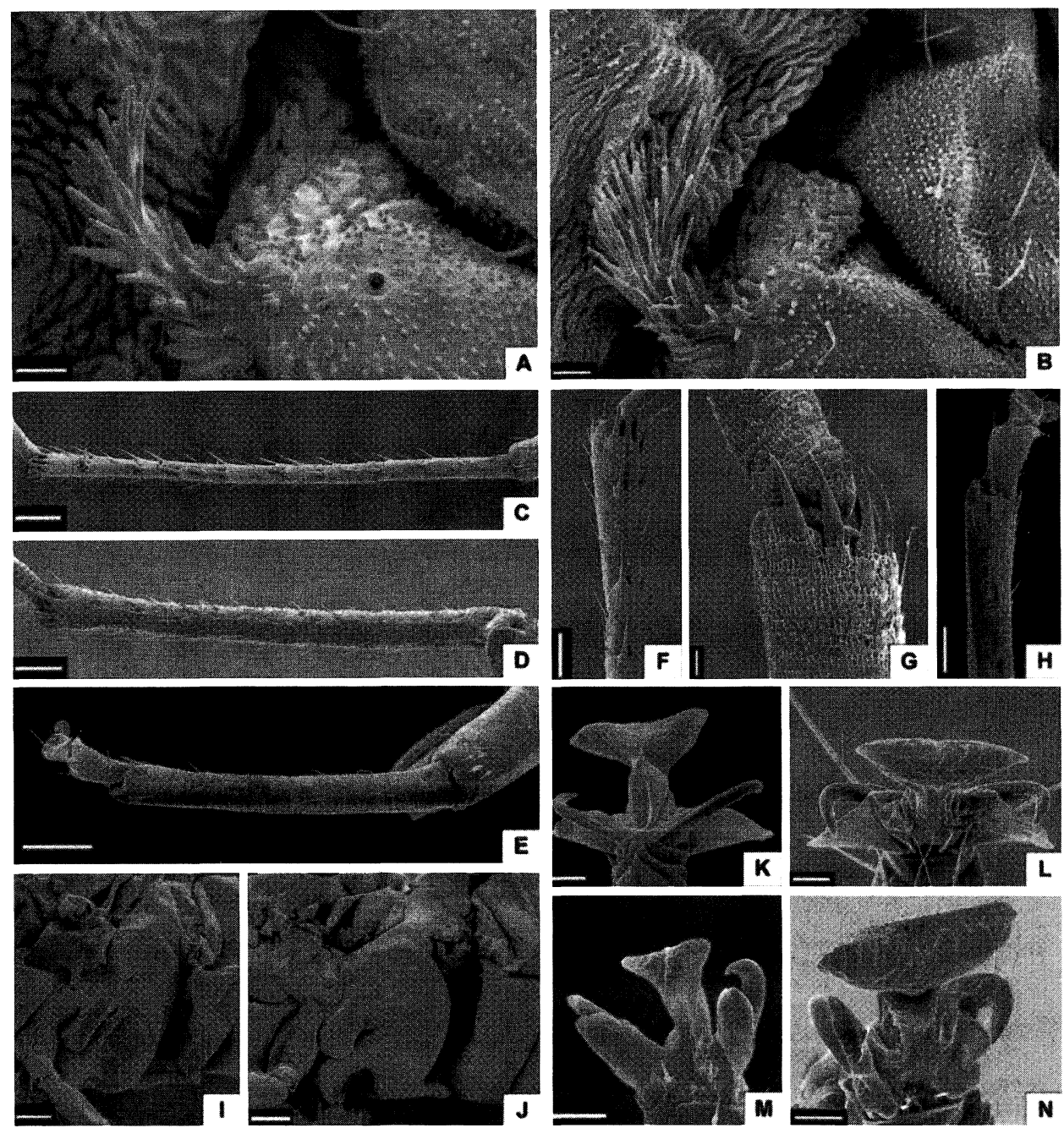

Fig. 4. Paurocephala spp.: (A-C, F, I, K, L) P. psylloptera; (D, G) P. hollisi; (E, H, J) P. cf. brevicephala; (M) P. chonchaiensis; (N) P. sulawesiana. (A, B) Patch on first visible abdominal tergite, lateral view $\left(\mathrm{A}+\mathrm{B}_{0}\right) ;(\mathrm{C}-\mathrm{E})$ metatibia, lateral view; $(\mathrm{F}-\mathrm{H})$ apex of metatibia; (I, J) metacoxa, lateral view; (K) fifth instar larval tarsal arolium, dorsal view; (L-N) fifth instar larval tarsal arolium, ventral view. Scale bar: A, B, G, K-N, $0.01 \mathrm{~mm}$; C, E, I, J, $0.1 \mathrm{~mm}$; D, F, H, $0.05 \mathrm{~mm}$.

and $P$. brevicephala, but in $P$. setifera and other species the flagellum is incompletely subdivided. Only in $P$. boehmeriae and $P$. maculipennis proper segmentation is present. Presence and shape of sectasetae, simple setae or lanceolate setae on different parts of the dorsum are also important in defining species. Most larvae do not have a humeral lobe, but this is developed (figure 45) in species such as $P$. brevicephala. The shape of the caudal plate is rounded in species as $P$. brevicephala and relatively long in others, while all Afrotropical species have distinct long tubercle-like extensions marginally (figures 35, 36). The position of the anus can be either ventral (figure 35 ) as in P. gossypii Russell or terminal (figures 39, 40) as in P. artocarpae. The outer circumanal ring is composed of one row of pores (figure 35), which in 


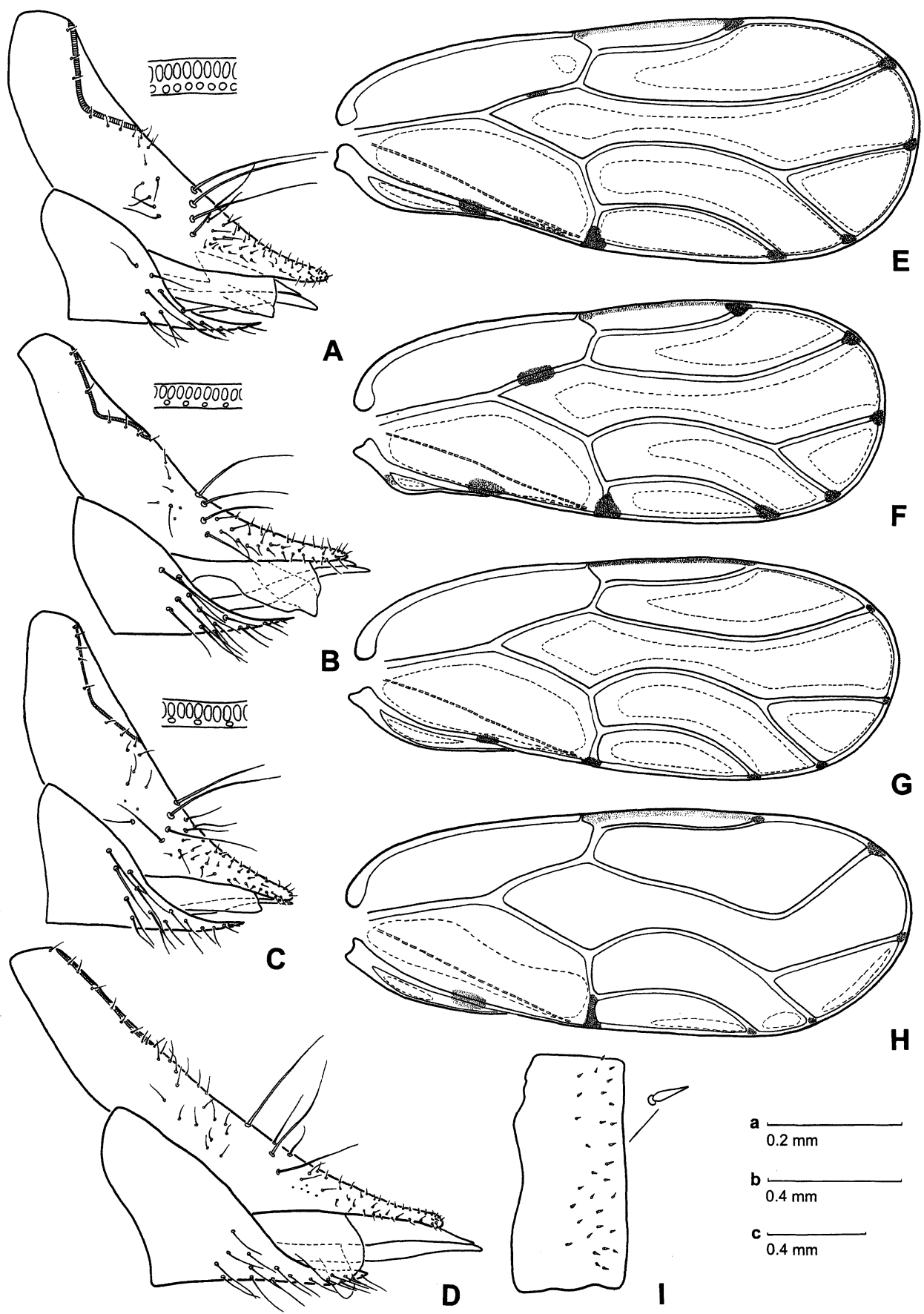

Fig. 5. Paurocephala spp.: (A, E) P. abutili; (B, F) P. gossypii; (C, G) P. urenae; (D, H, I) $P$. lucida. (A-D) Female genitalia, lateral view (A-C including section of female circumanal ring, outer pores above); $(\mathrm{E}-\mathrm{H})$ forewing; (I) third visible abdominal tergite, lateral view. Scale bar: a, A-D, I; b, E-G; c, H. 


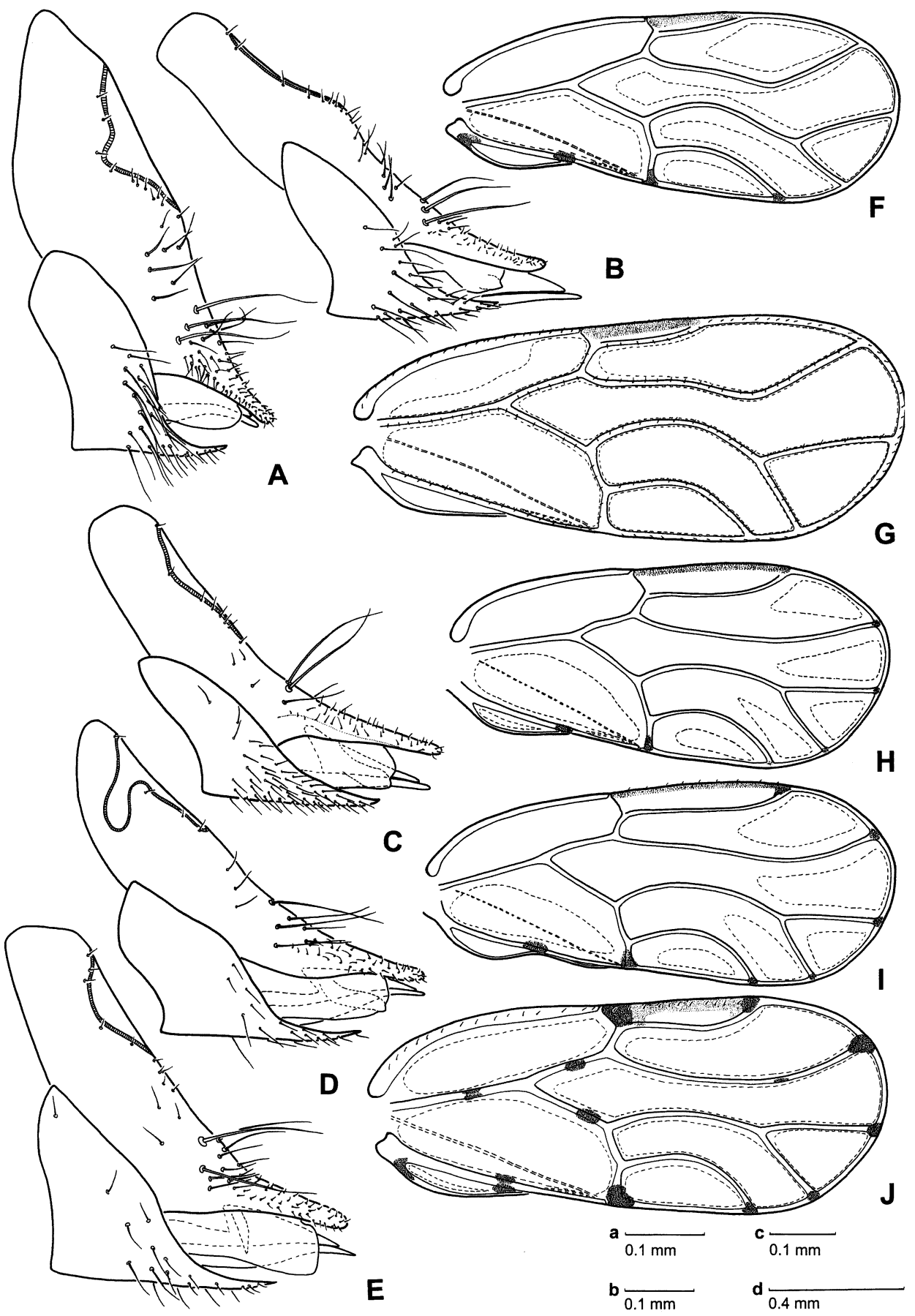

Fig. 6. Paurocephala spp.: (A, F) P. hollisi; (B, G) P. sinuata; (C, H) P. boxi; (D, I) P. medleri; (E, J) P. insolita. (A-E) Female genitalia, lateral view; ( $\mathrm{F}-\mathrm{J})$ forewing. Scale bar: a, A, B; b, C, D; c, E; d, F-J. 

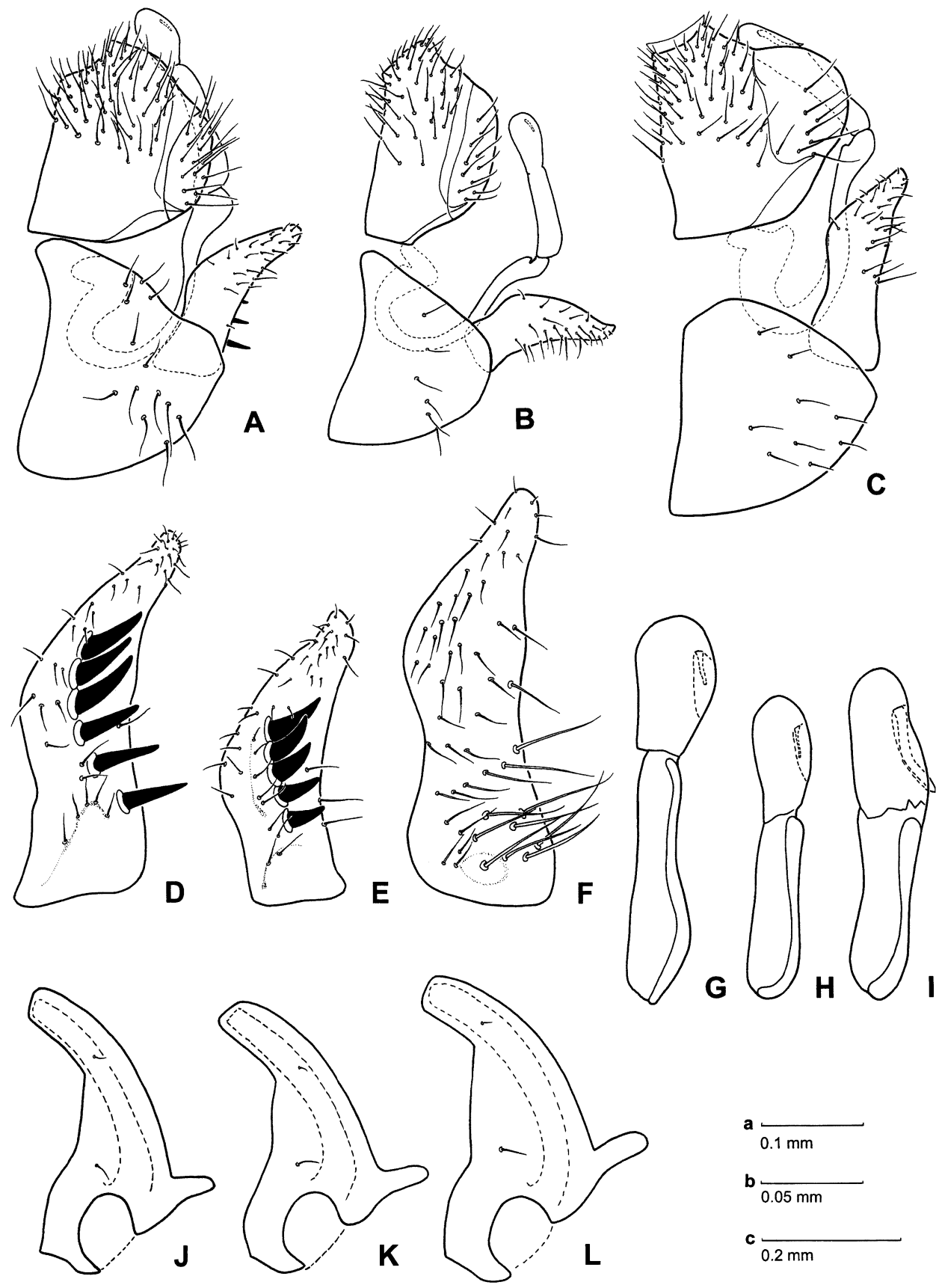

Fig. 7. Paurocephala spp.: (A, D, G, J) P. hollisi; (B, E, H, K) P. sinuata; (C, F, I, L) P. insolita. (A-C) Male genitalia, lateral view; (D-F) paramere, inner surface; $(\mathrm{G}-\mathrm{I})$ distal segment of aedeagus; (J-L) metacoxa, lateral view. Scale bar: a, A-C; b, D-I; c, J-L. 


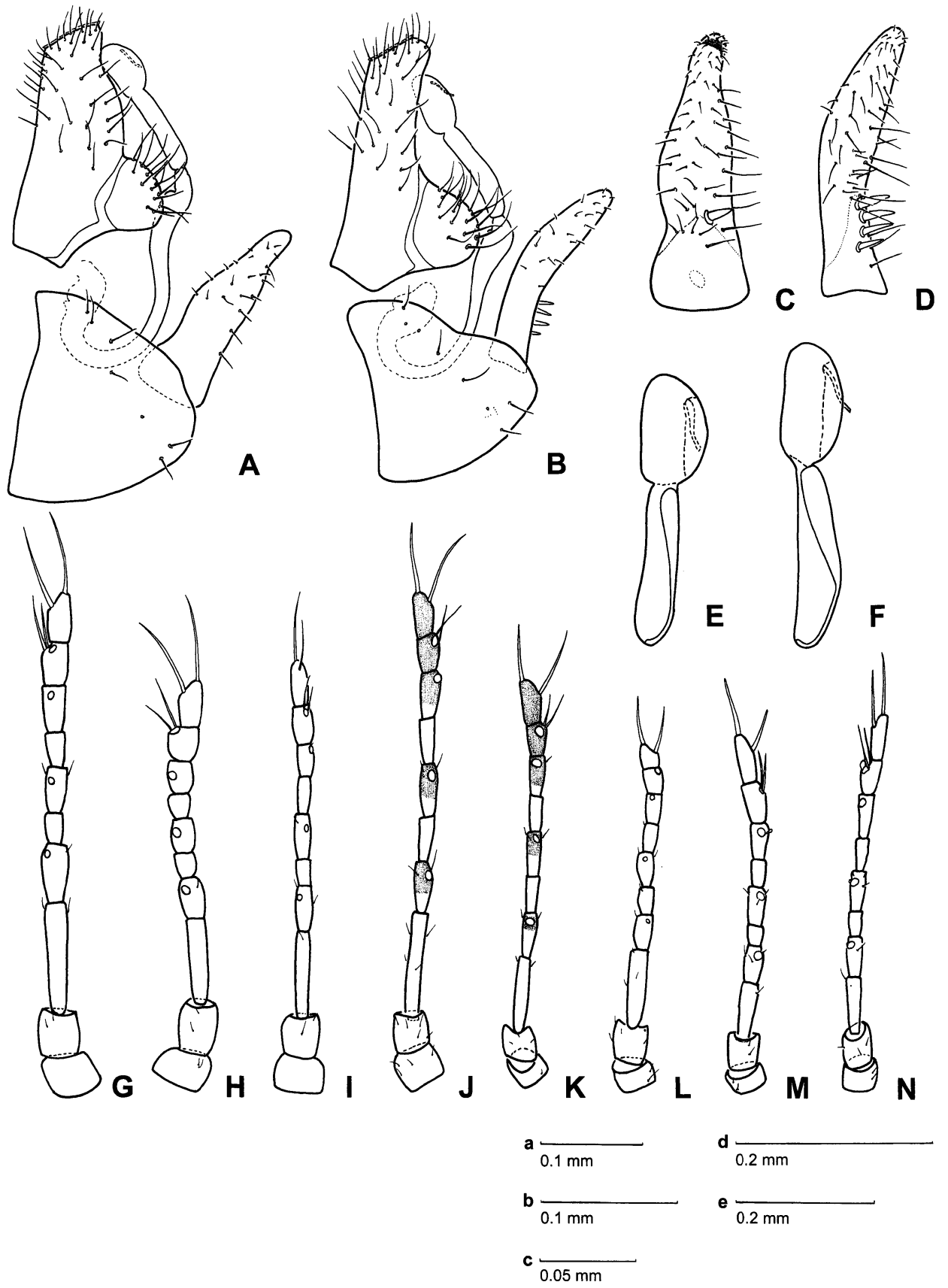

Fig. 8. Paurocephala spp.: (A, C, E, M) P. boxi; (B, D, F, N) P. medleri; (G) P. abutili; (H) P. gossypii; (I) P. urenae; (J) P. hollisi; (K) P. sinuata; (L) P. insolita. (A, B) Male genitalia, lateral view; $(C, D)$ paramere, inner surface; $(E, F)$ distal segment of aedeagus; (G-N) antenna. Scale bar: a, A, B; b, C, D; c, E, F; d, G-I; e, J-N. 

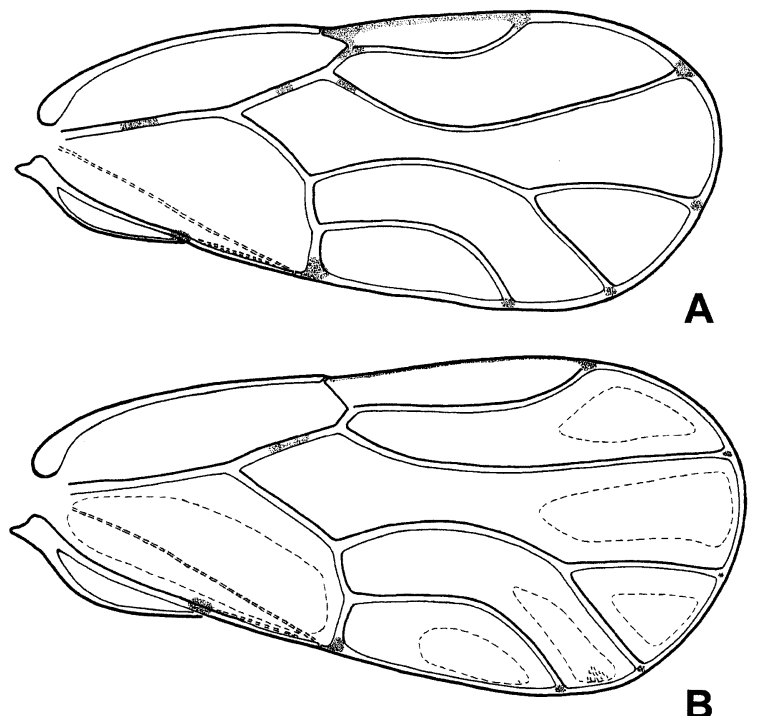

B
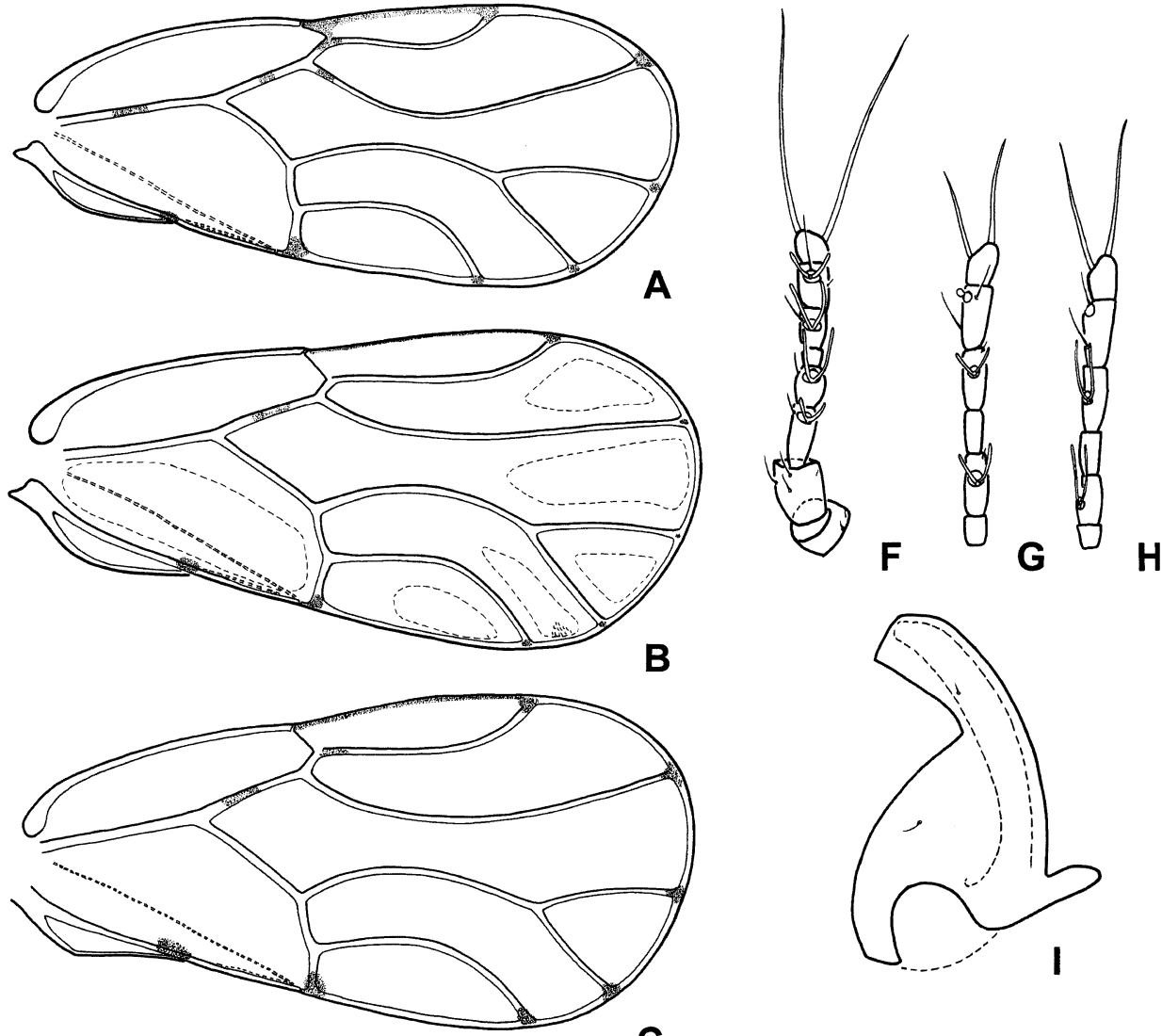

C

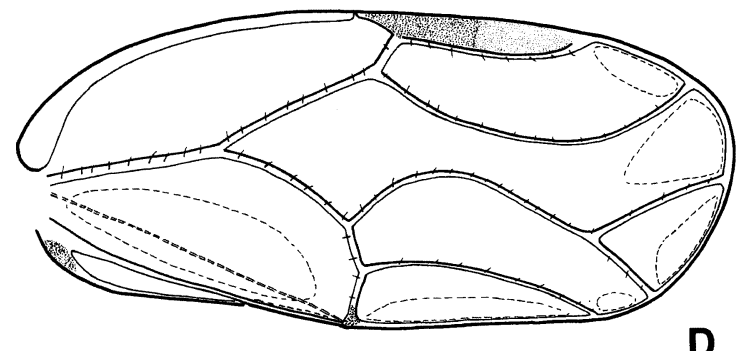

D
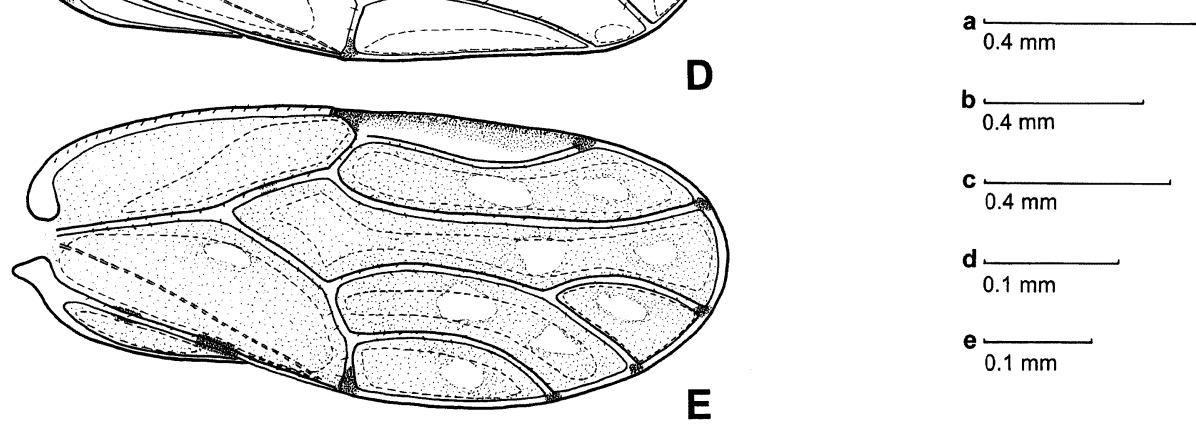

FIG. 9. Paurocephala spp.: (A, F) P. elegans; (B, G, I) P. kleinhofiae (Taiwan); (C, H) P. kleinhofiae (Philippines); (D) P. lienhardi; (E) P. brendelli. (A-E) forewing; (F) antenna; (G, H) distal segments of antenna; (I) metacoxa. Scale bar: a, A-C; b, D; c, $\mathrm{E} ; \mathrm{d}, \mathrm{F}-\mathrm{H} ; \mathrm{e}, \mathrm{I}$. 


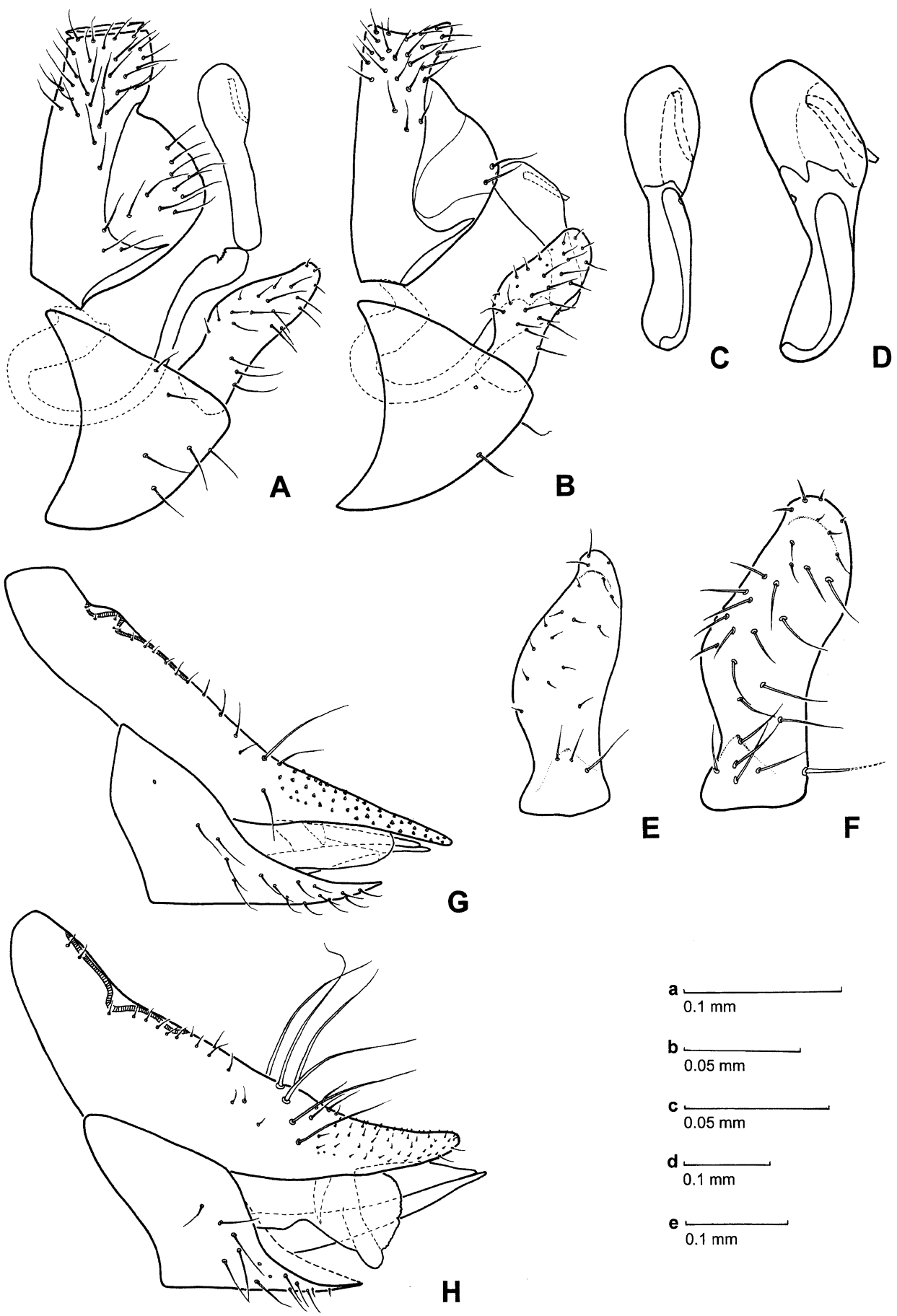

Fig. 10. Paurocephala spp.: (A, C, E, G) P. elegans; (B, D, F, H) P. kleinhofiae (Taiwan). (A, B) Male genitalia, lateral view; (C, D) distal segment of aedeagus; (E, F) paramere, inner surface; $(\mathrm{G}, \mathrm{H})$ female genitalia, lateral view. Scale bar: a, A, B; b, C, E; c, D, F; d, G; e, H. 


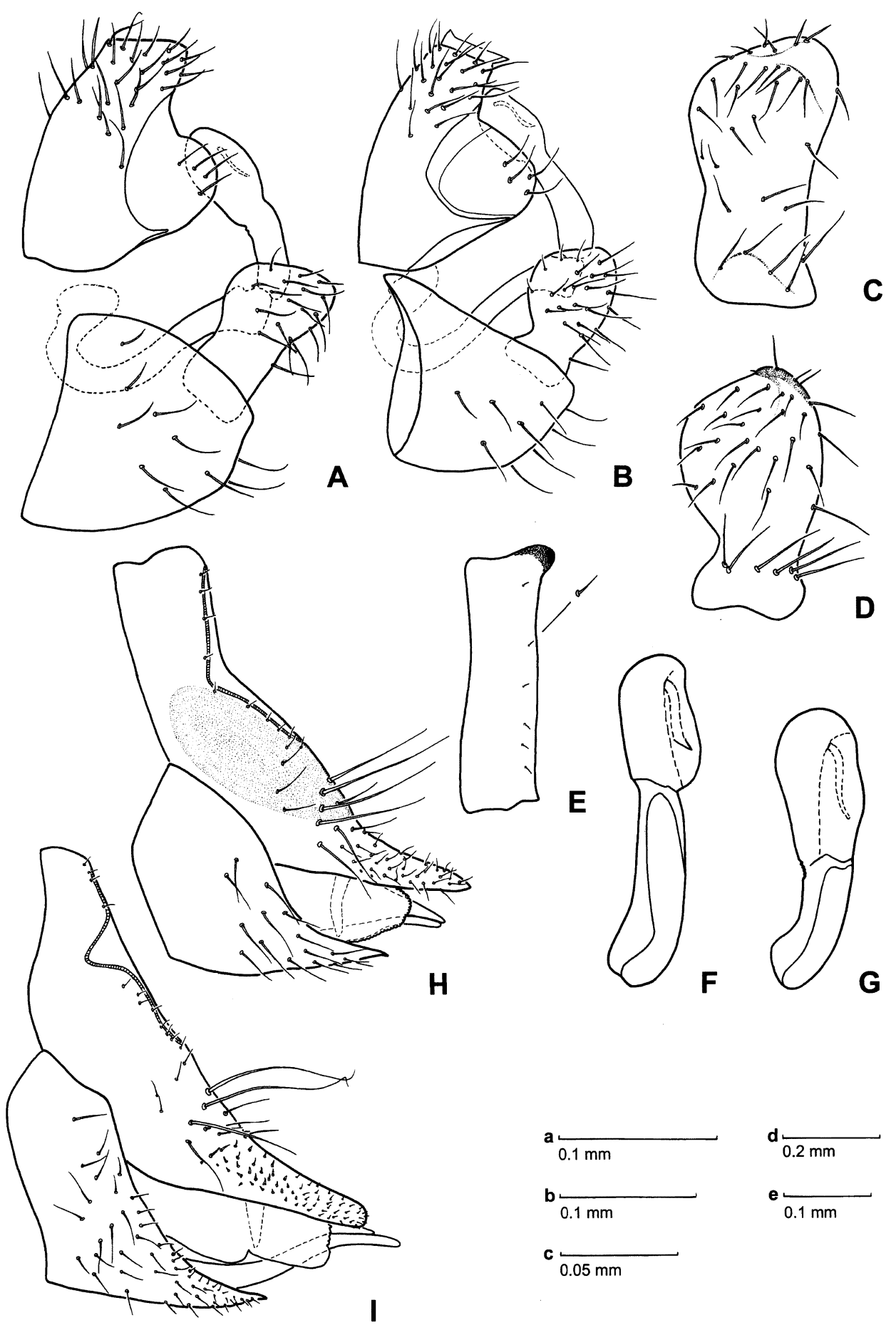

FIG. 11. Paurocephala spp.: (A, C, F, H) P. lienhardi; (B, D, E, G, I) P. brendelli. (A, B) Male genitalia, lateral view; (C, D) paramere, inner surface; (E) third visible abdominal tergite, lateral view; $(F, G)$ distal segment of aedeagus; $(H, I)$ female genitalia, lateral view. Scale bar: a, A; b, B; c, C, D, F, G; d, E; e, H, I. 

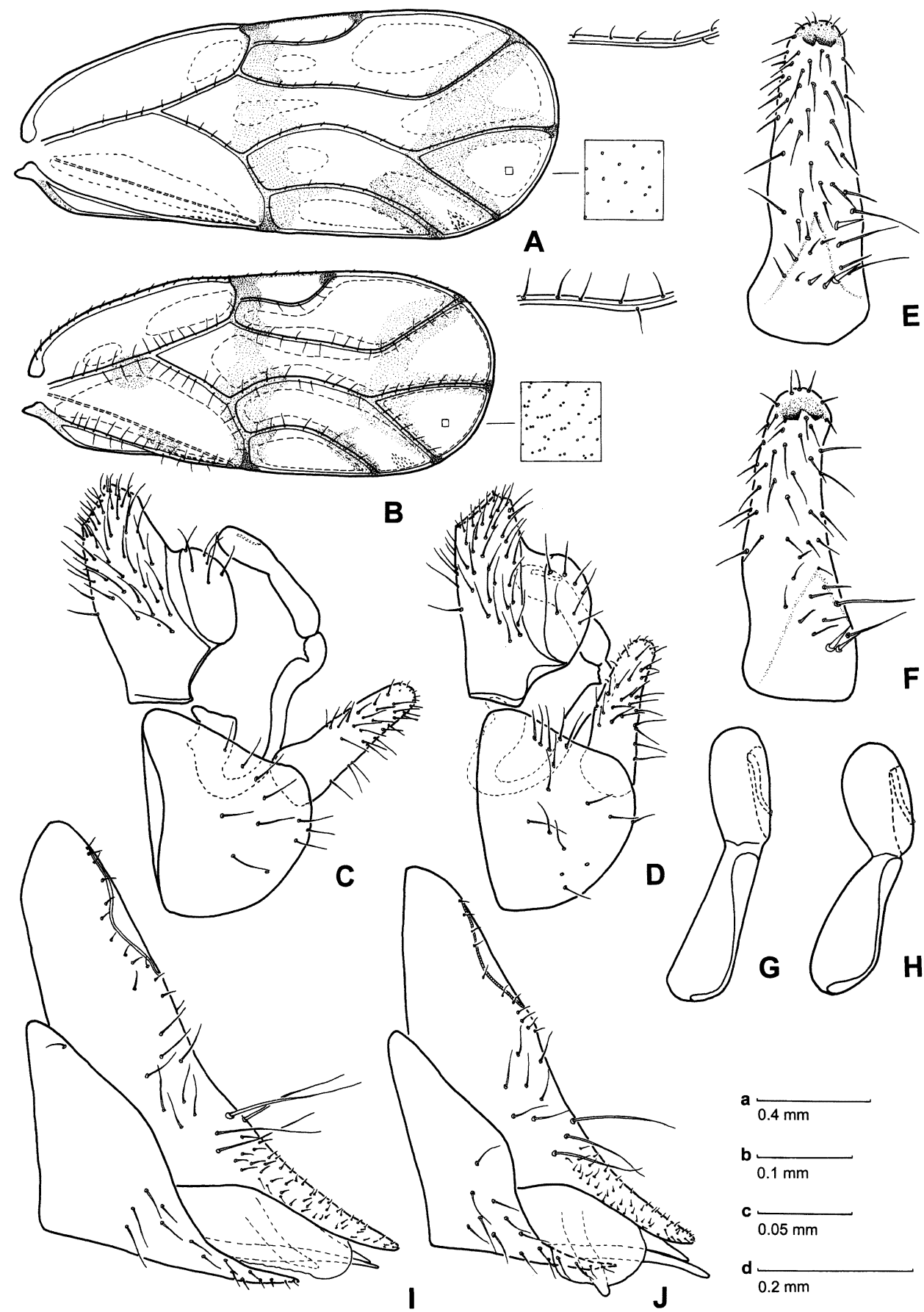

Fig. 12. Paurocephala spp.: (A, C, E, G, I) P. bifasciata; (B, D, F, H, J) P. chonchaiensis. (A, B) Forewing (including detail of middle part of Rs vein, and surface spinules in cell $\left.\mathrm{m}_{1}\right) ;(\mathrm{C}, \mathrm{D})$ male genitalia, lateral view; $(\mathrm{E}, \mathrm{F})$ paramere, inner surface; $(\mathrm{G}, \mathrm{H})$ distal segment of aedeagus; (I, J) female genitalia, lateral view. Scale bar: a, A, B; b, C, D; c, E-H; d, I, J. 

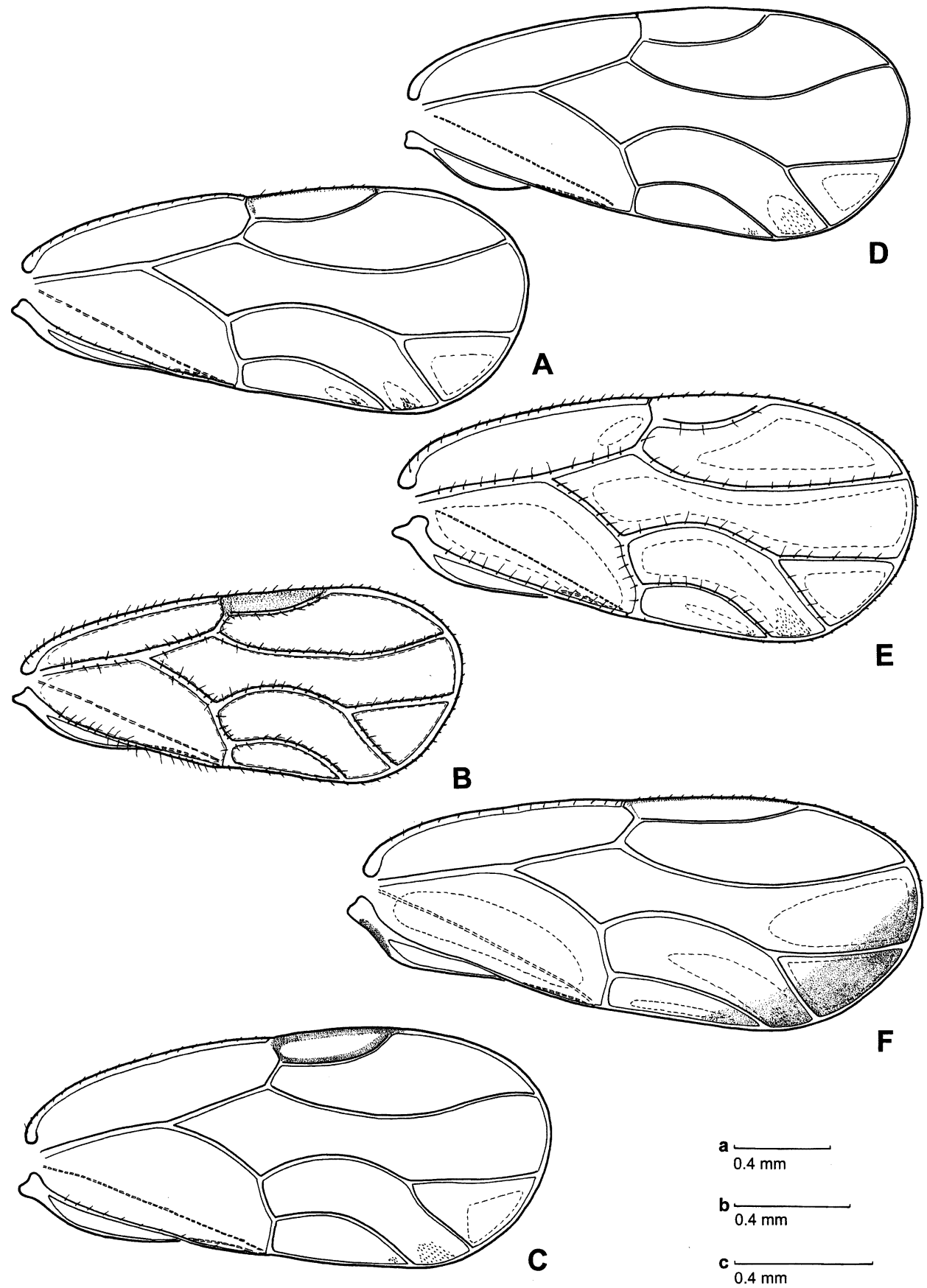

FIG. 13. Paurocephala spp.: (A) P. similis; (B) P. artocarpae; (C) P. curvata; (D) P. calcarata; (E) P. javanica; (F) P. wilderi. (A-F) Forewing. Scale bar: a, A, B; b, C-E; c, F.

some species is expanded laterally (figures 37,38 ) as in $P$. curvata and $P$. chonchaiensis, or with additional pores at irregular intervals (figure 39 ) as in P. artocarpae. The arolium is distinctly expanded basally in several species (figure $4 \mathrm{~K}-\mathrm{N}$ ). 


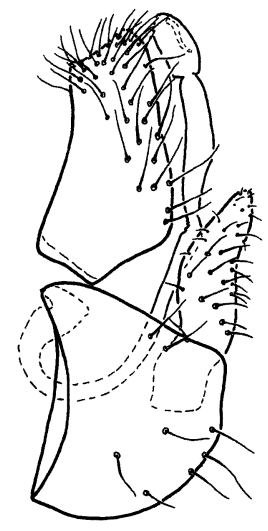

A
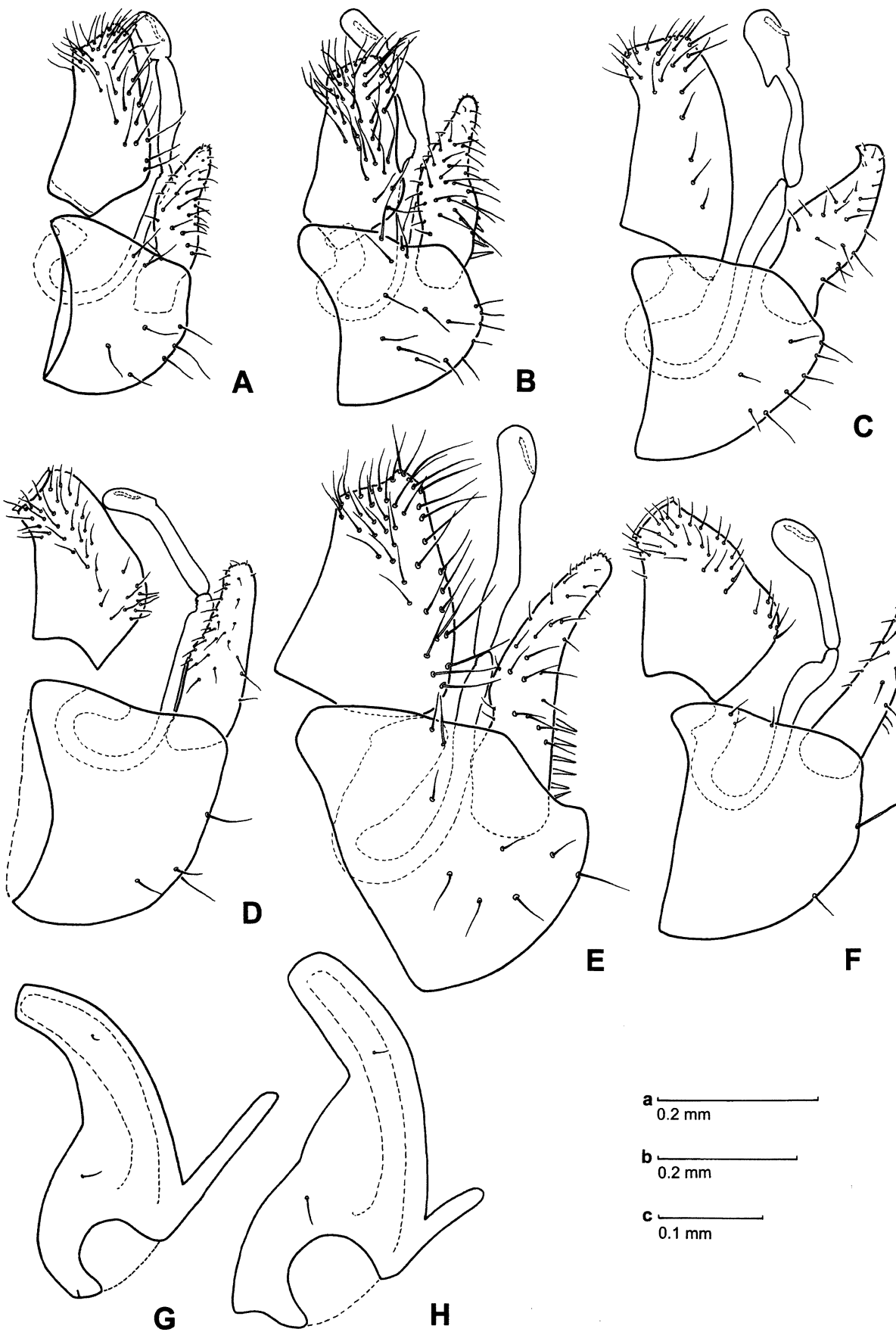

$\mathbf{E}$
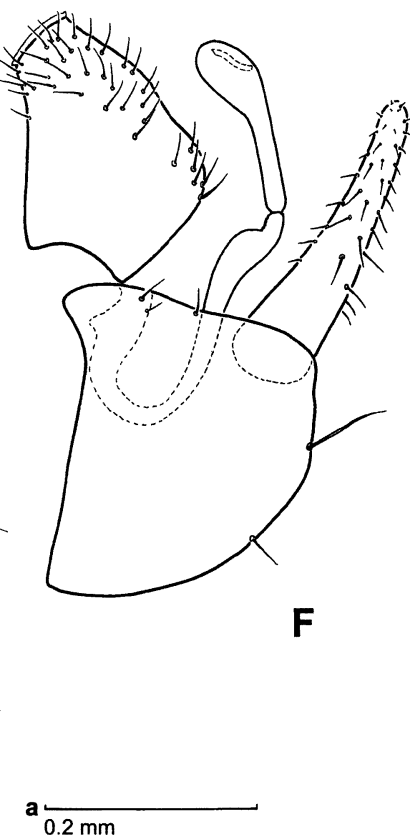

b

C $\overline{0.1 \mathrm{~mm}}$

Fig. 14. Paurocephala spp.: (A) P. similis; (B) P. artocarpae; (C, H) P. curvata; (D, G) $P$. calcarata; (E) P. javanica; (F) P. wilderi. (A-F) Male genitalia, lateral view; $(\mathrm{G}-\mathrm{H})$ metacoxa. Scale bar: a, A; b, B, G, H; c, C-F. 

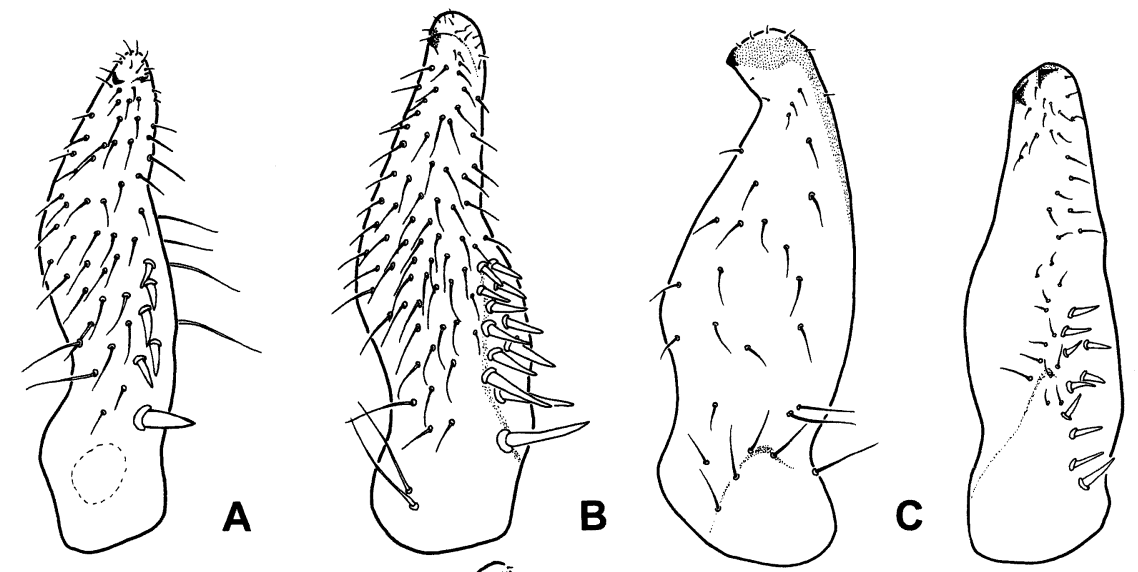

D

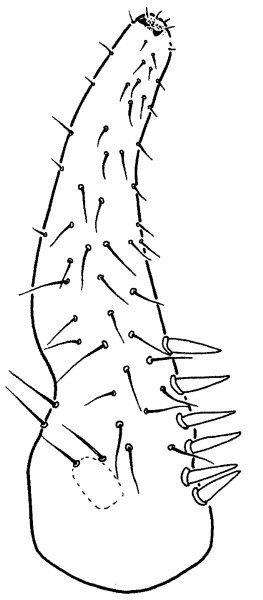

E

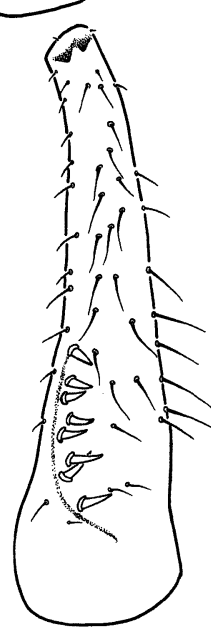

F
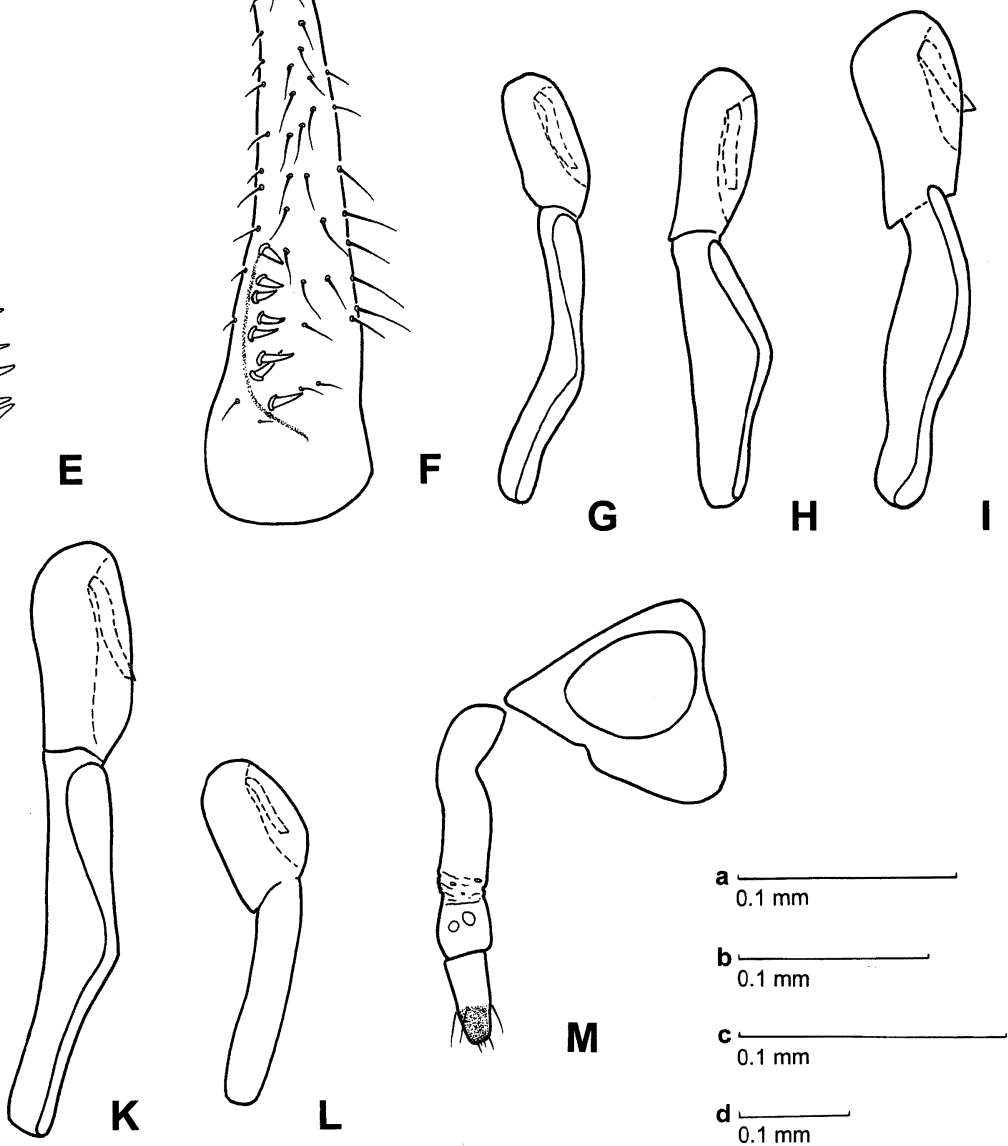

FIG. 15. Paurocephala spp.: (A, G) P. similis; (B, H) P. artocarpae; (C, I, M) P. curvata; (D, J) P. calcarata; (E, K) P. javanica; (F, L) P. wilderi. (A-F) Paramere, inner surface; (G-L) distal segment of aedeagus; (M) clypeus and labium, lateral view. Scale bar: a, A, G; b, B, E, H; c, C, D, F, I-K; d, M. 


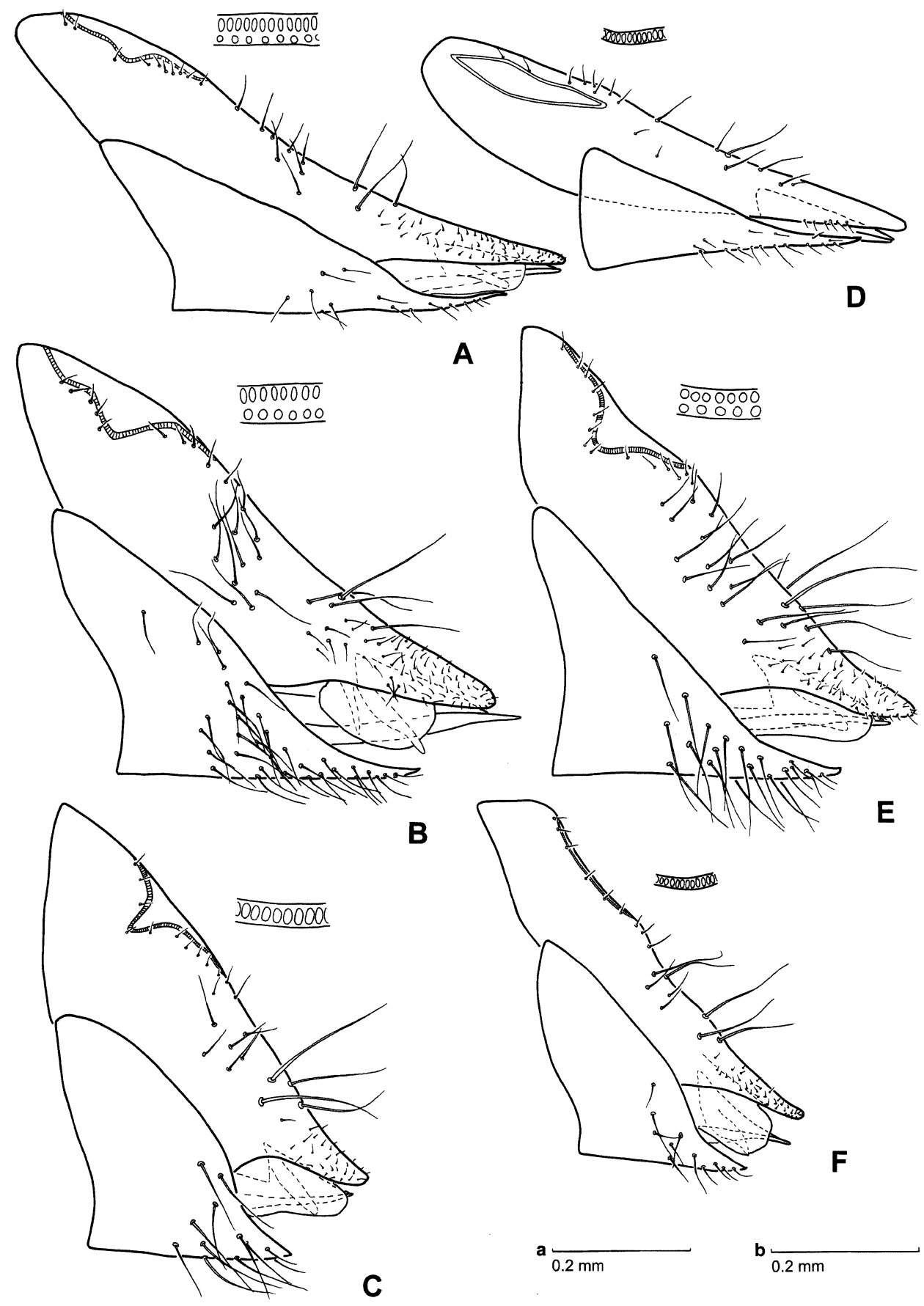

Fig. 16. Paurocephala spp.: (A) P. similis; (B) P. artocarpae; (C) P. curvata; (D) P. calcarata; (E) P. javanica; (F) P. wilderi. (A-F) Female genitalia, lateral view (including section of female circumanal ring, outer pores above). Scale bar: a, A, B, E; b, C, D, F. 


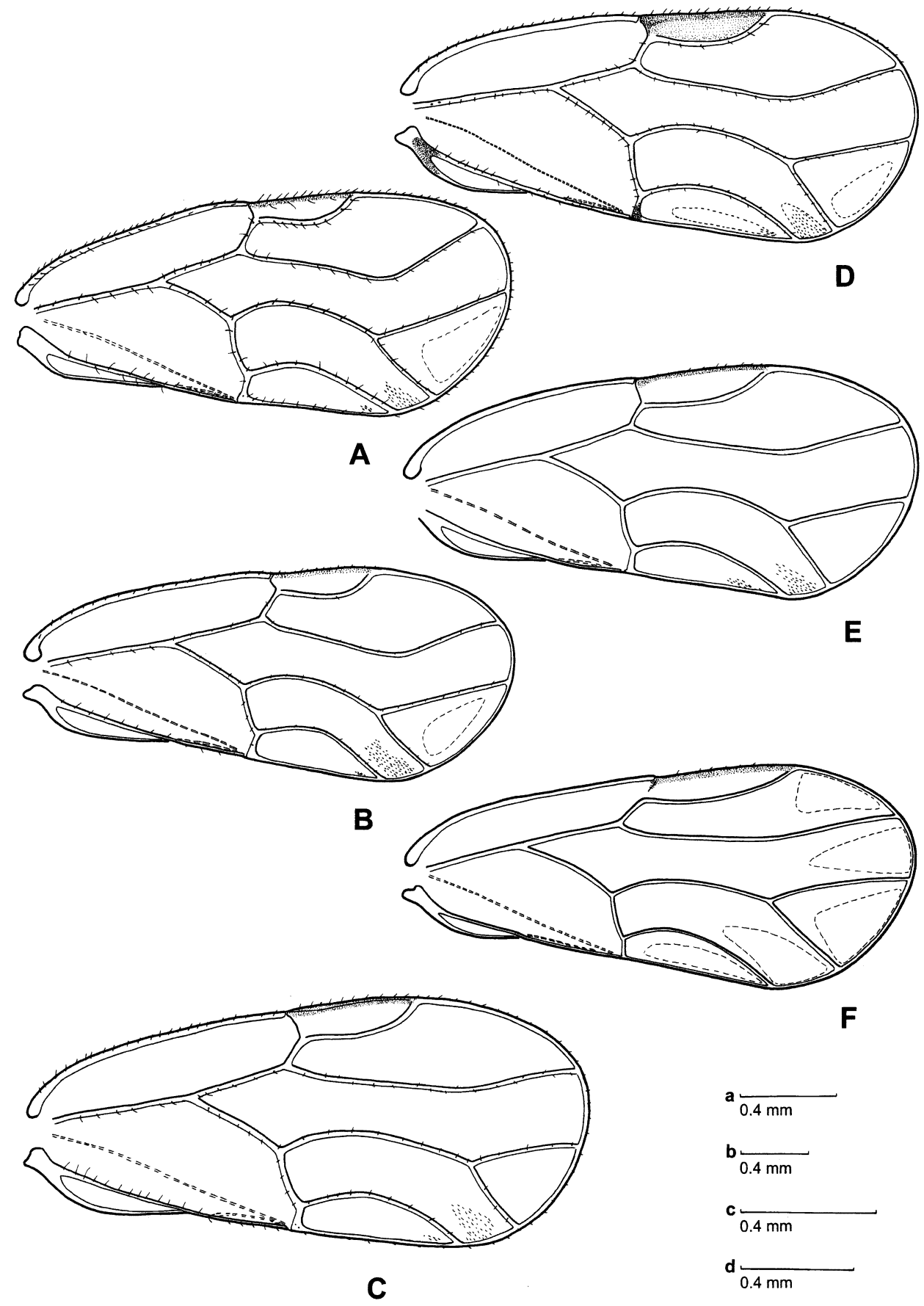

FIG. 17. Paurocephala spp.: (A) P. maculipennis; (B) P. longiantennata; (C) P. psylloptera; (D) P. muiri; (E) P. papuana; (F) P. distincta. (A-F) Forewing. Scale bar: a, A, F; b, B; c, C, D; d, E. 


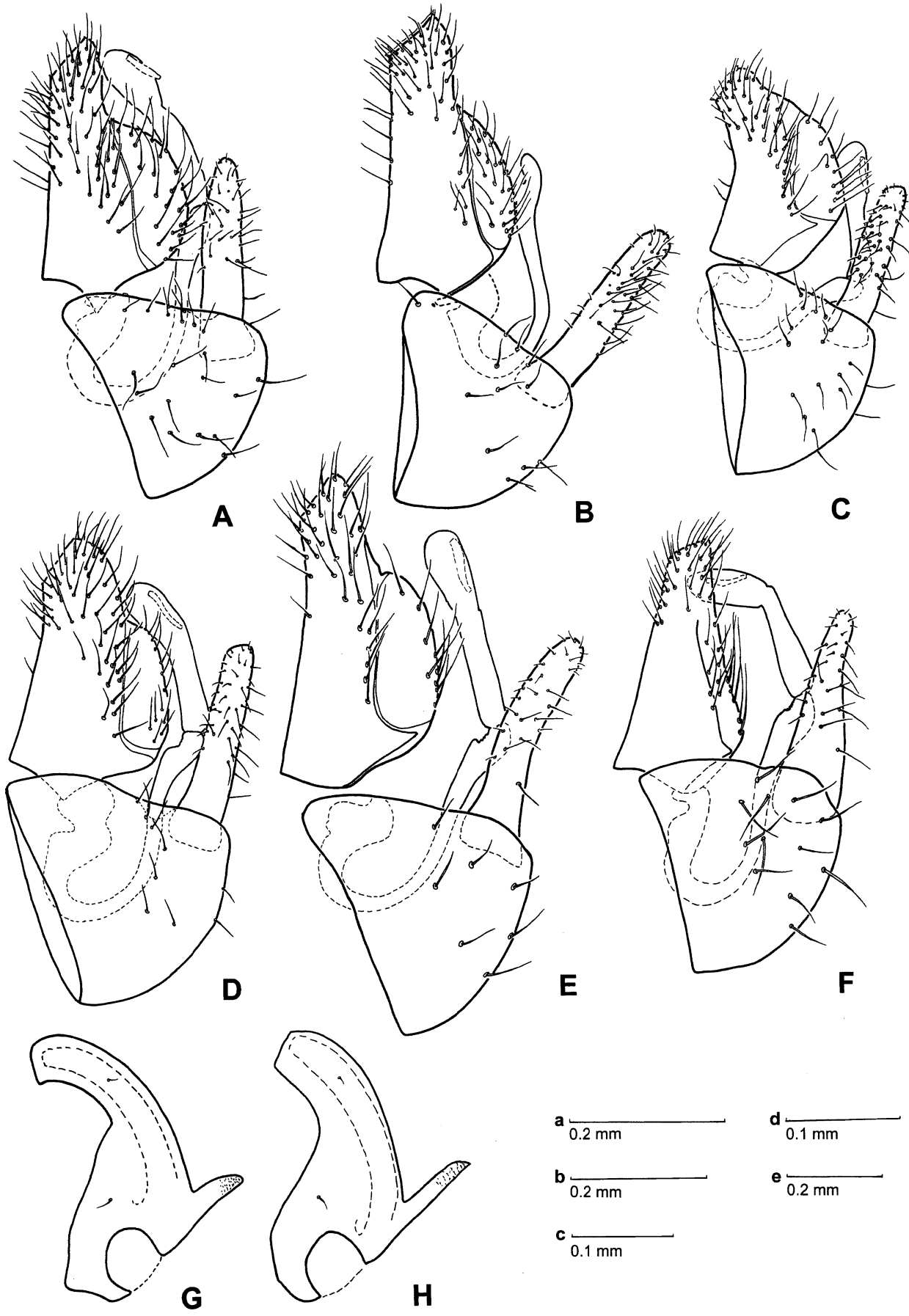

FIG. 18. Paurocephala spp.: (A, G) P. maculipennis; (B) P. longiantennata; (C, H) P. psylloptera; (D) P. muiri; (E) P. papuana; (F) P. distincta. (A-F) Male genitalia, lateral view; $(\mathrm{G}, \mathrm{H})$ metacoxa. Scale bar: a, A, C; b, B; c, D, F; d, E; e, G, H. 


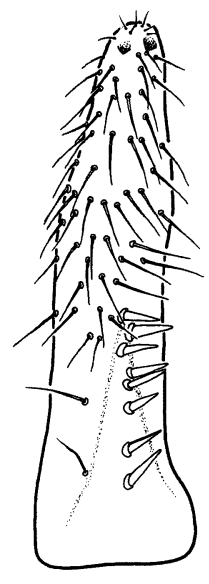

A

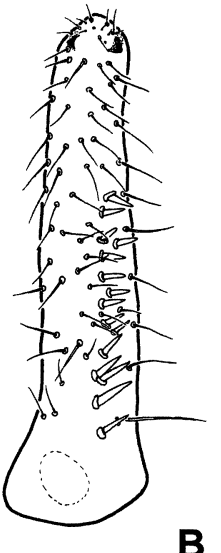

B

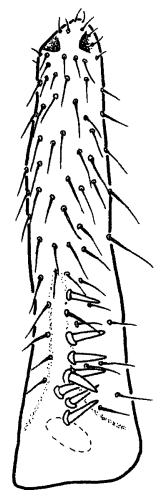

c
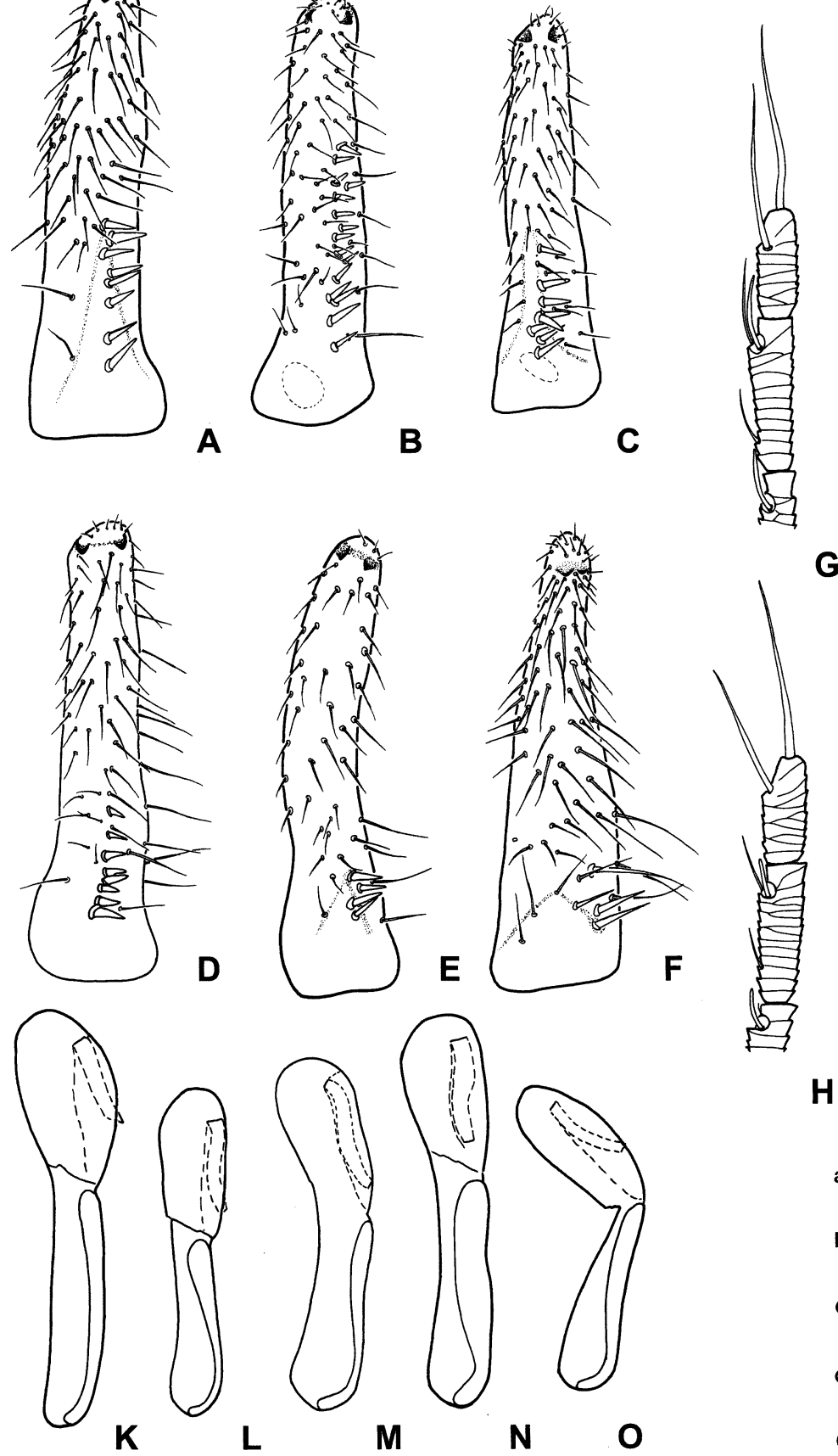

G
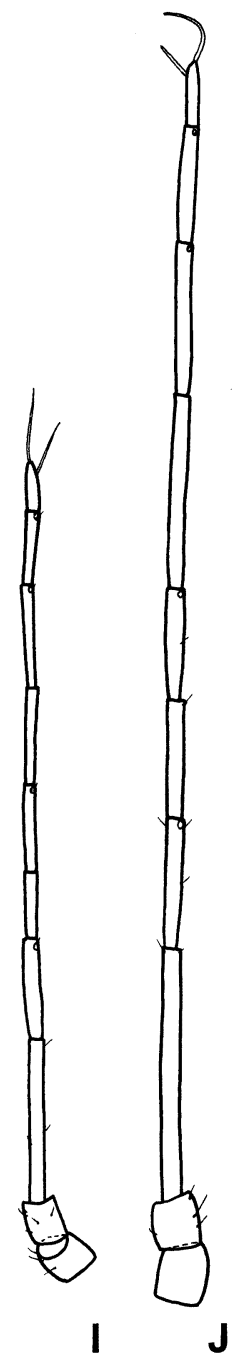

H

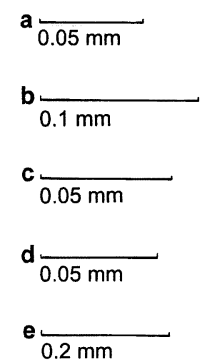

Fig. 19. Paurocephala spp.: (A, I, K) P. maculipennis; (B, J) P. longiantennata; (C, G, L) P. psylloptera; (D, H, M) P. muiri; (E, N) P. papuana; (F, O) P. distincta. (A-F) Paramere, inner surface; $(\mathrm{G}, \mathrm{H})$ distal segments of antenna; (I, J) antenna; (K-O) distal segment of aedeagus. Scale bar: a, A, C, G, H, K, L, O; b, B; c, D, E, M, N; d, F; e, I, J. 


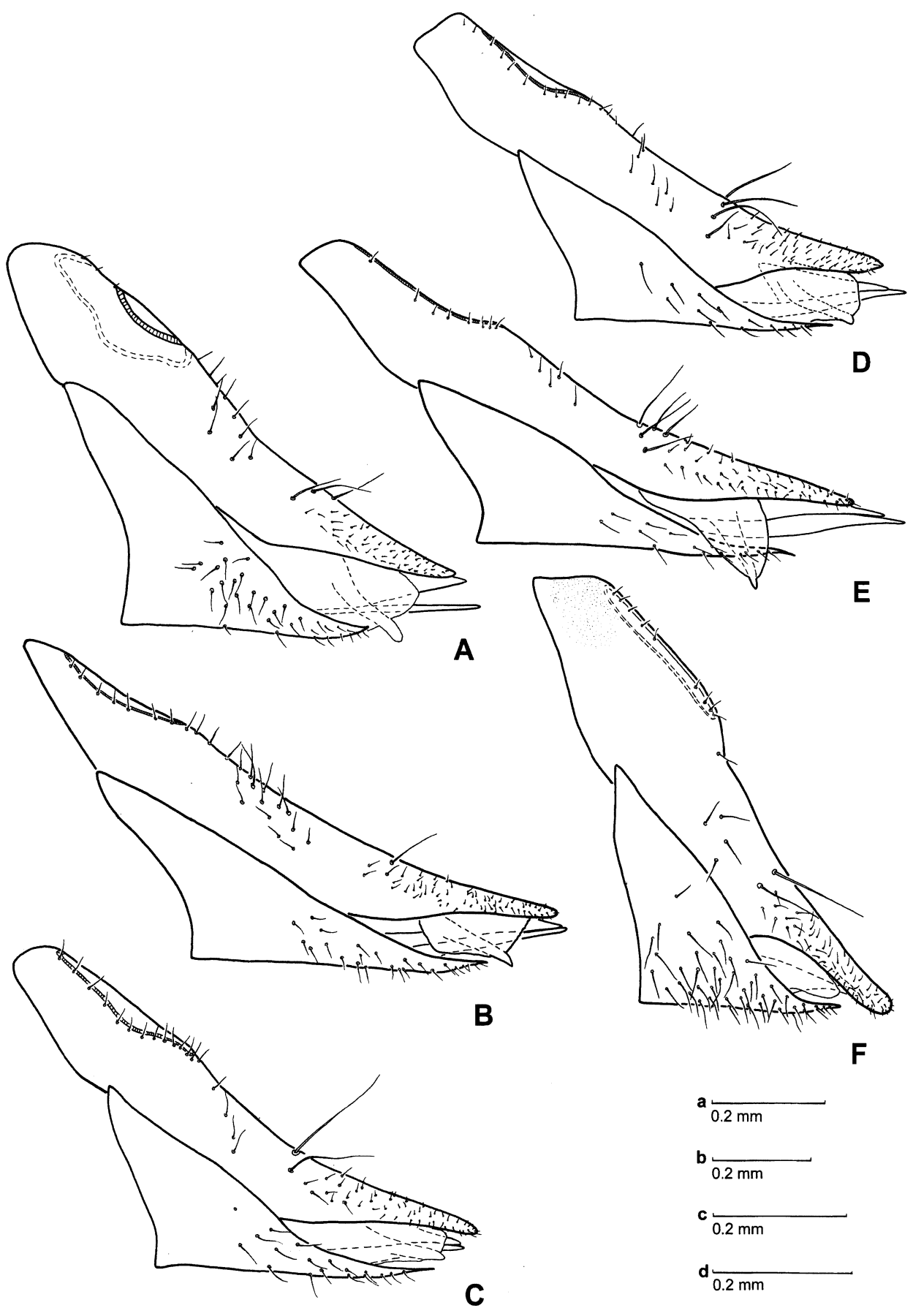

Fig. 20. Paurocephala spp.: (A) P. maculipennis; (B) P. longiantennata; (C) P. psylloptera; (D) P. muiri; (E) P. papuana; (F) P. distincta. (A-F) Female genitalia, lateral view. Scale bar: a, A; b, B; c, C, E, F; d, D. 

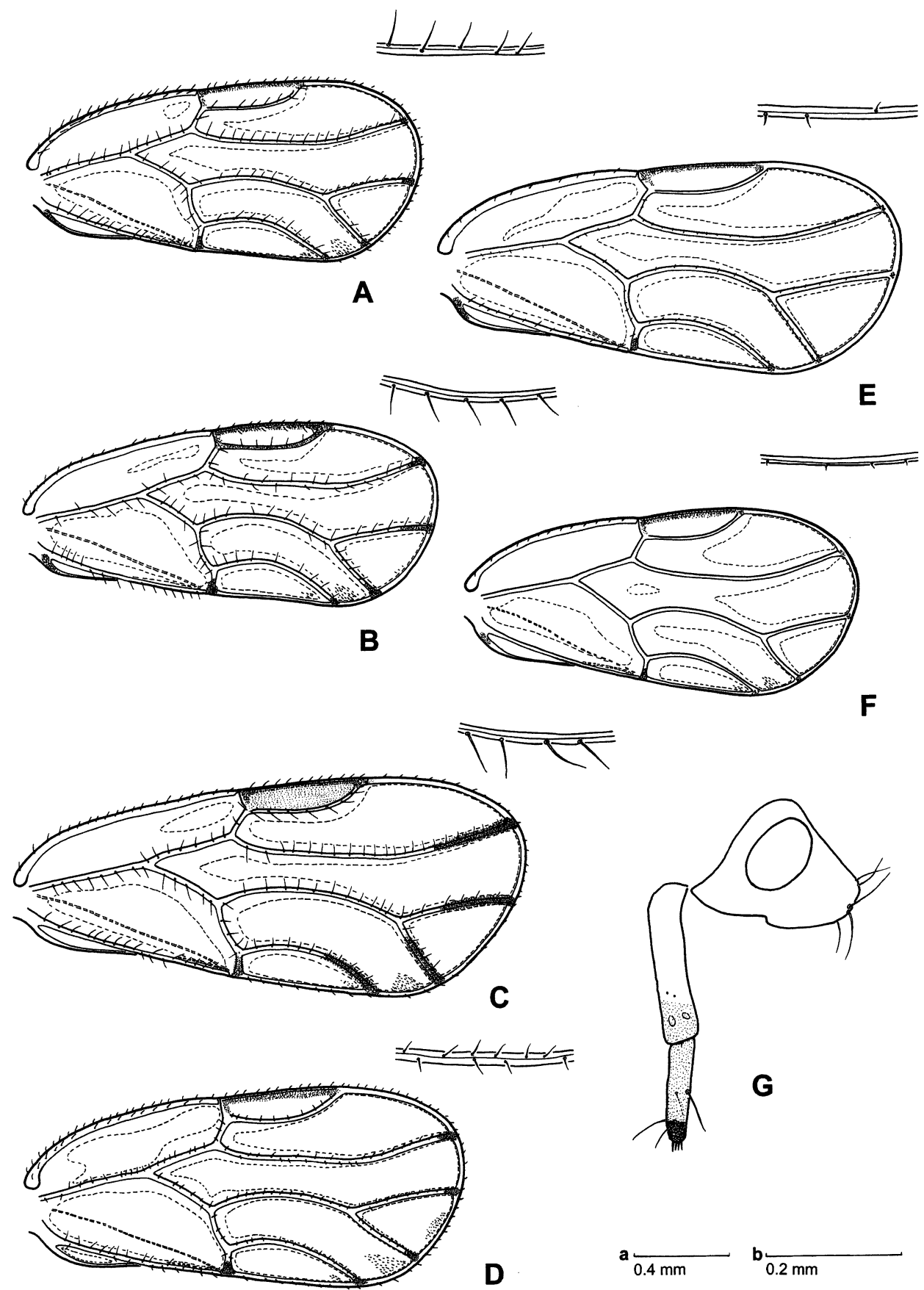

FIG. 21. Paurocephala spp.: (A, G) P. dayak; (B) P. oceanica; (C) P. marginata; (D) P. palawanensis; (E) P. trematos (from Taiwan); (F) P. trematos (from Sarawak). (A-F) Forewing (including detail of middle part of Rs vein); $(\mathrm{G})$ clypeus and labium, lateral view. Scale bar: a, A-F; b, G. 

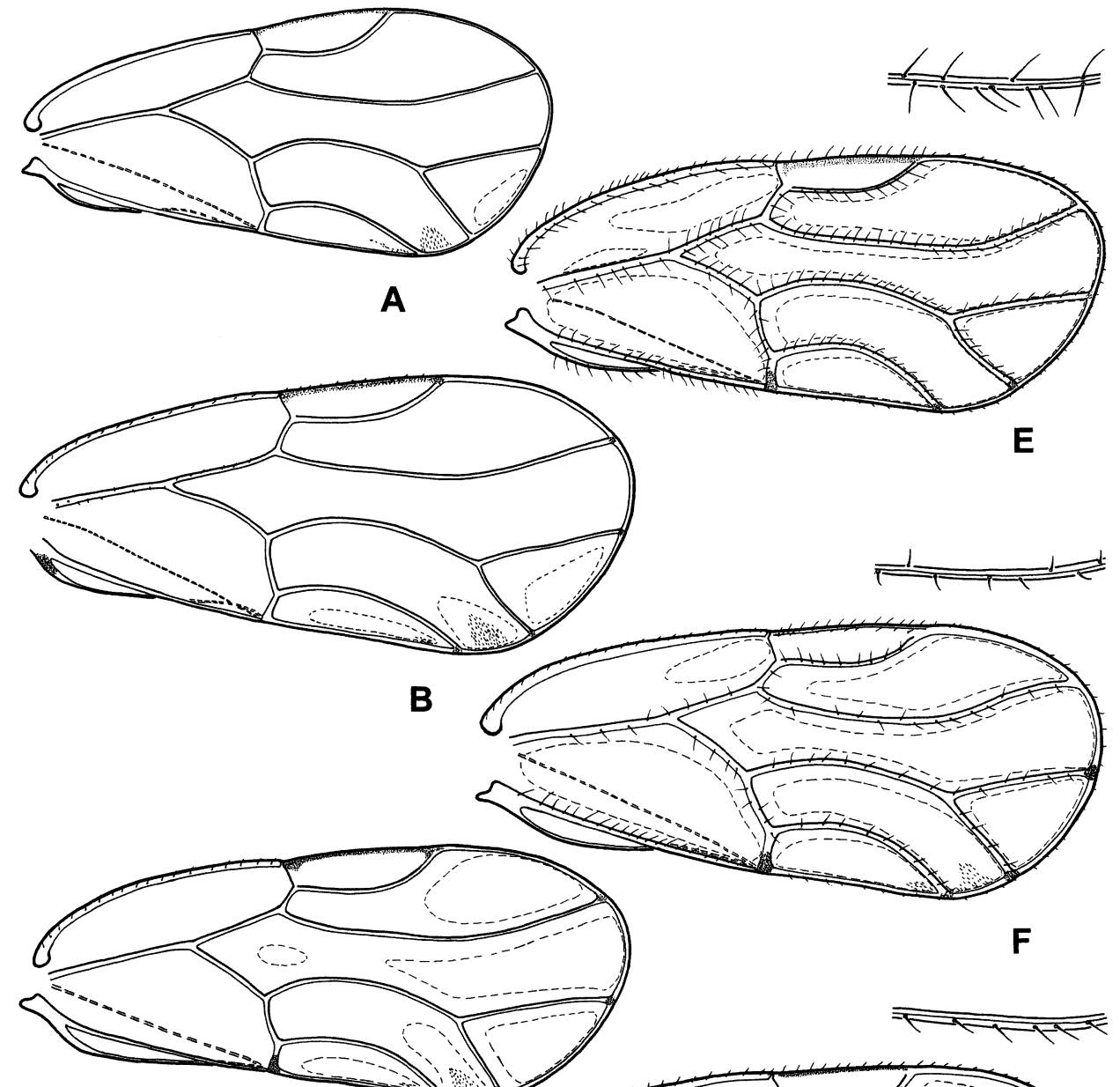

c
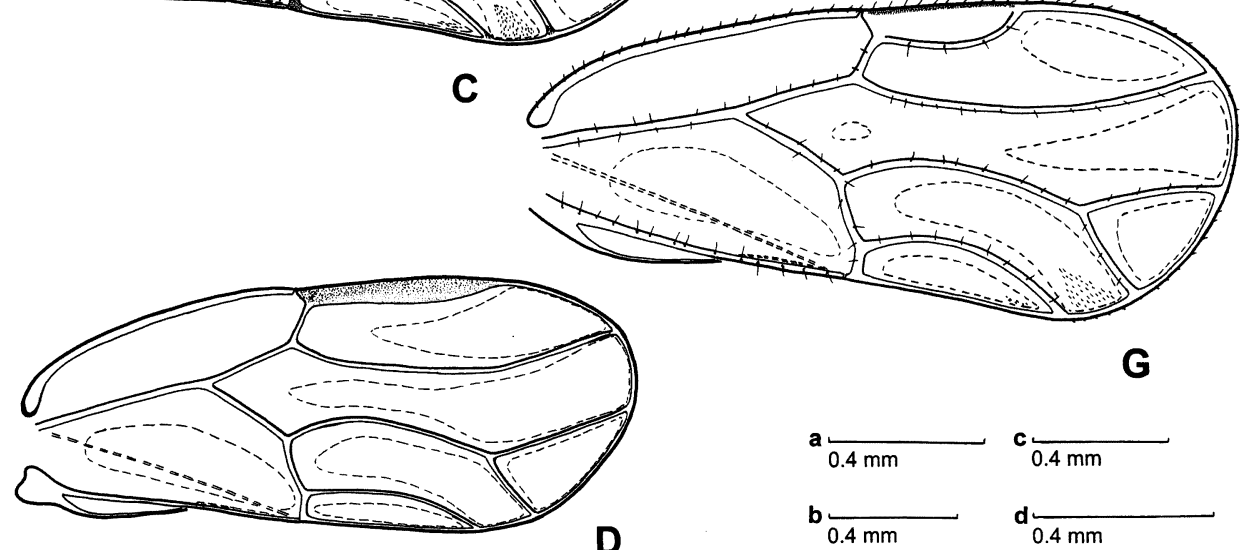

G

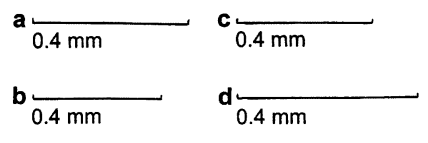

FIG. 22. Paurocephala spp.: (A) P. macrochaetis; (B) P. sauteri; (C) P. boehmeriae; (D) P. stigmaticalis; (E) P. setifera; (F) P. polaszeki; (G) P. muta. (A-G) Forewing (E-G including details of middle part of Rs vein). Scale bar: a, A; b, B; c, C-E; d, F, G. 


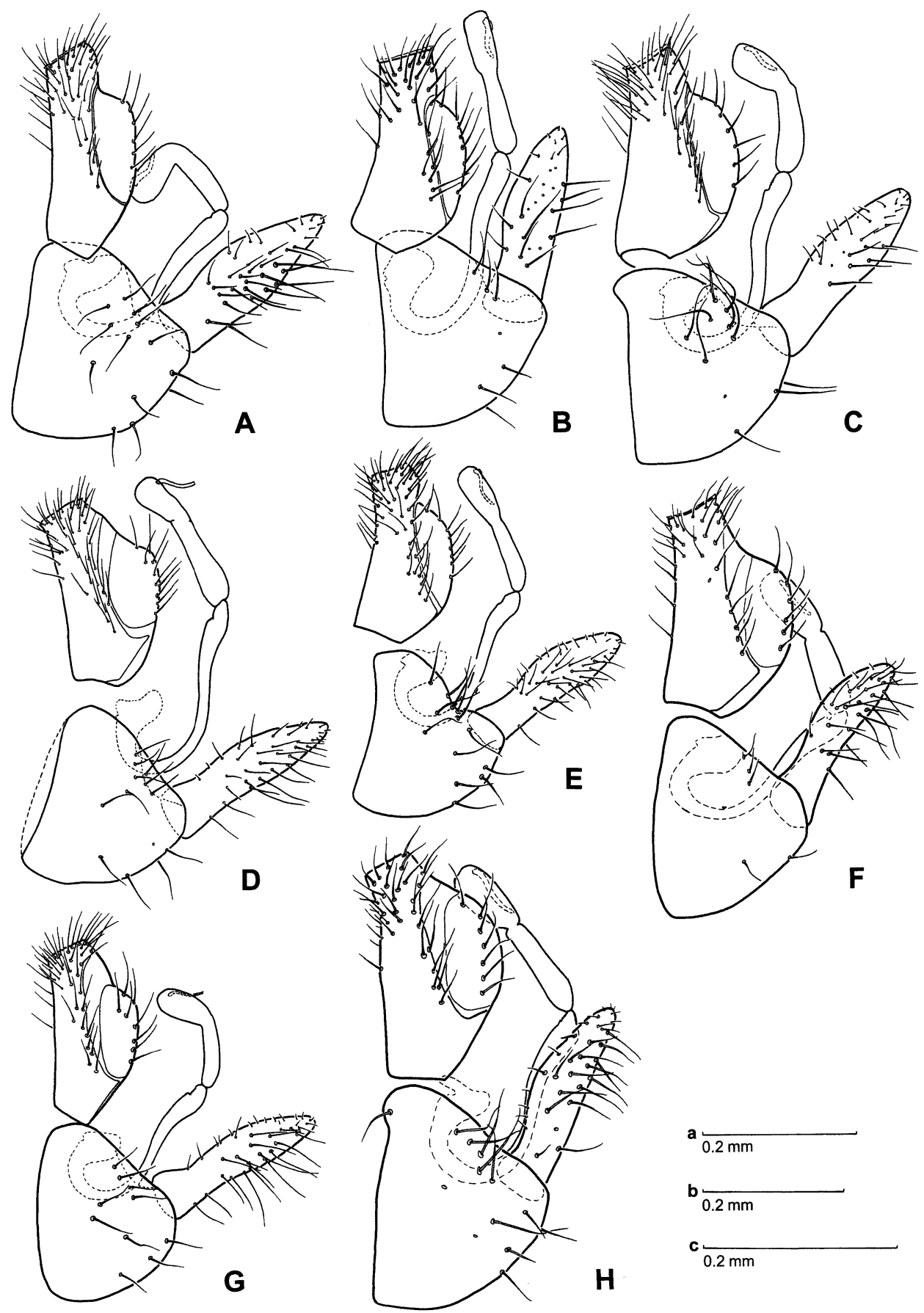

FIg. 23. Paurocephala spp.: (A) P. dayak; (B) P. oceanica; (C) P. marginata; (D) P. palawanensis; (E) P. trematos; (F) P. macrochaetis; (G) P. sauteri; (H) P. boehmeriae. (A-H) Male genitalia, lateral view. Scale bar: a, A-D; b, E, G; c, F, H. 

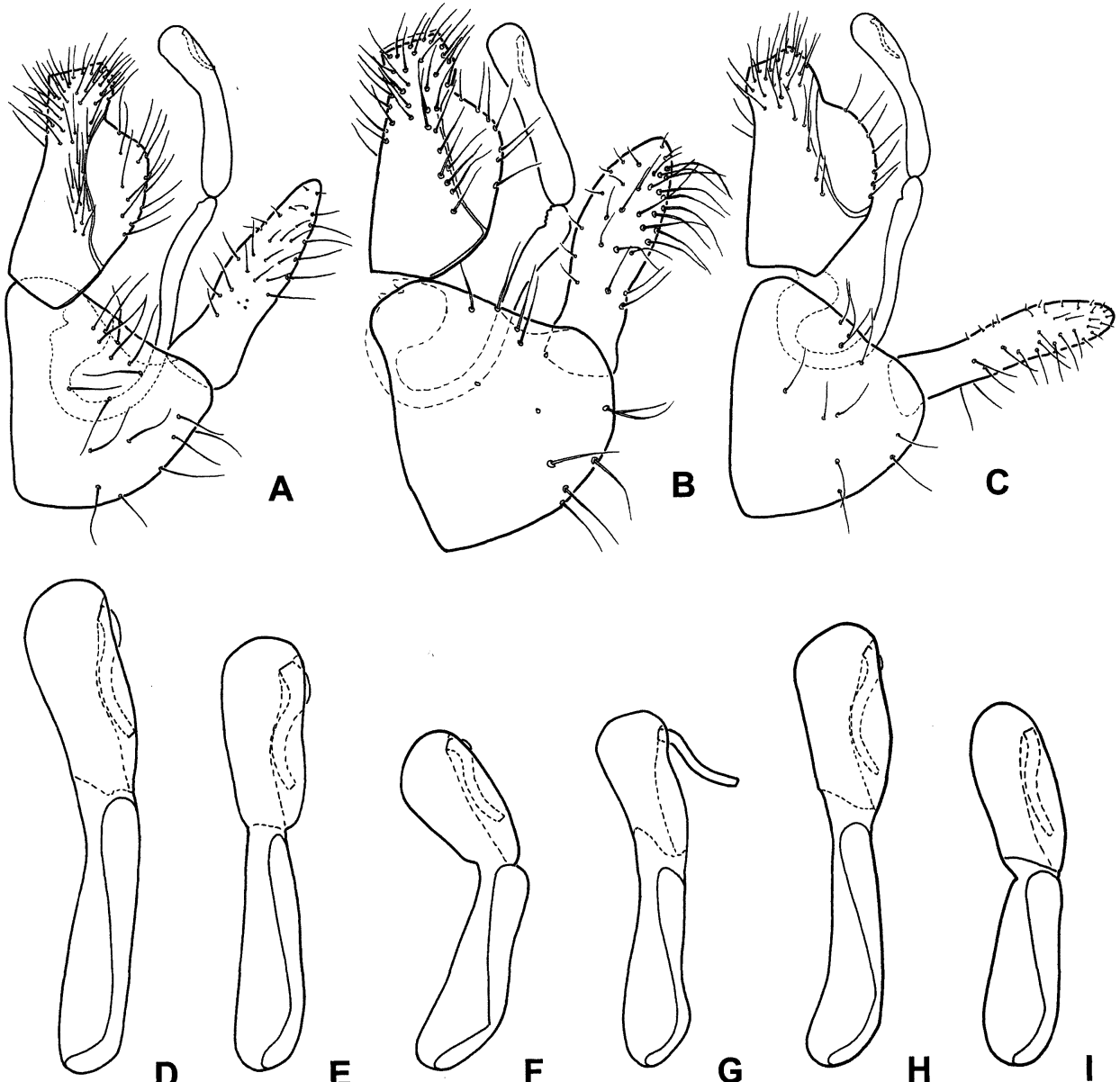

D

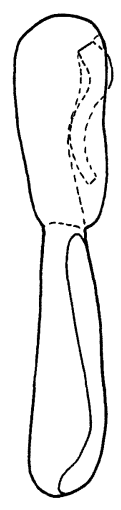

E
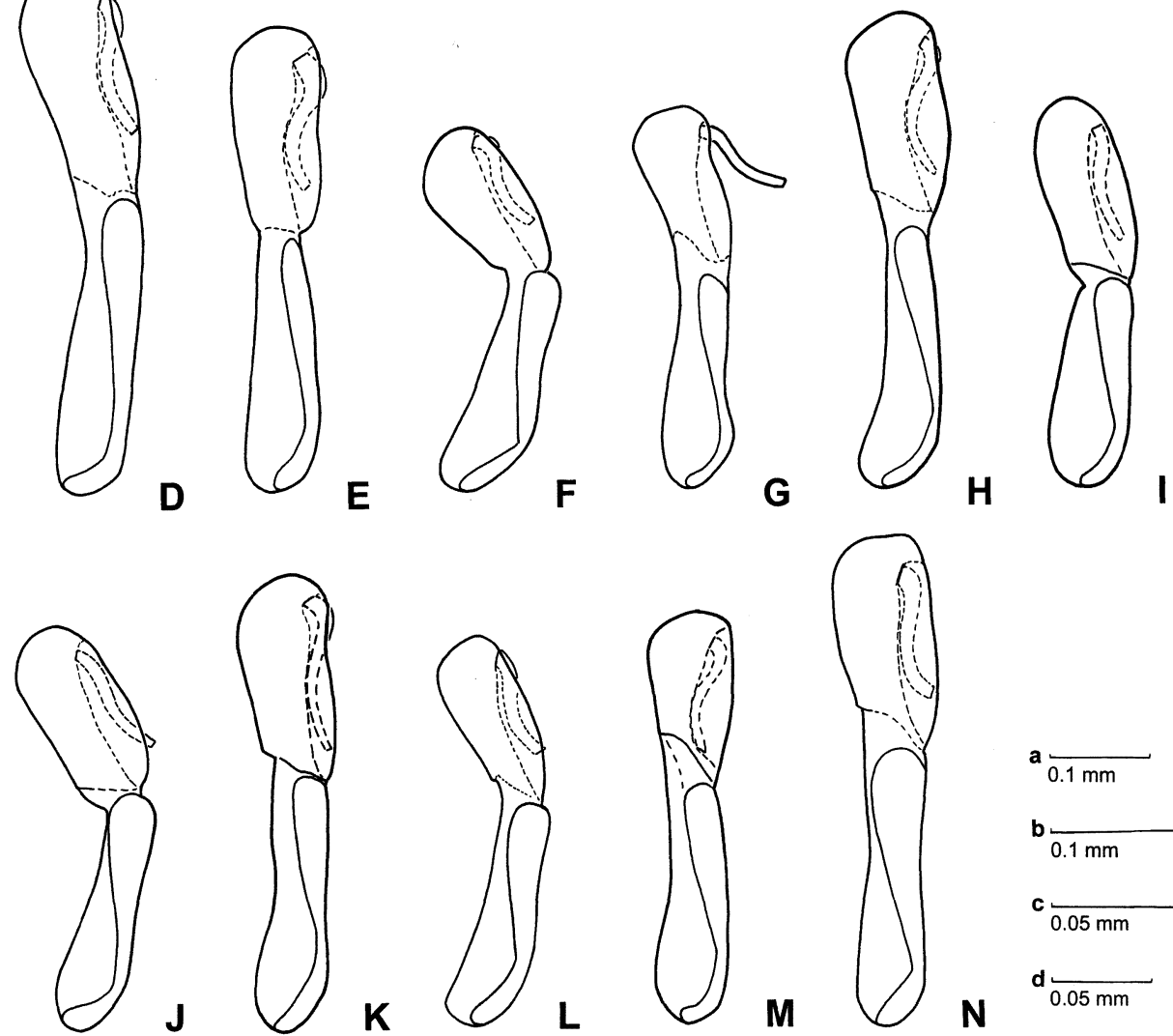

H

Fig. 24. Paurocephala spp.: (A, L) P. setifera; (B, M) P. polaszeki; (C, N) P. muta; (D) P. dayak; (E) P. oceanica; (F) P. marginata; (G) P. palawanensis; (H) P. trematos; (I) P. macrochaetis; (J) P. sauteri; (K) P. boehmeriae. (A-C) Male genitalia, lateral view; (D-N) distal segment of aedeagus. Scale bar: a, A, C; b, B; c, D, E, H-K, M, N; d, F, G, L. 

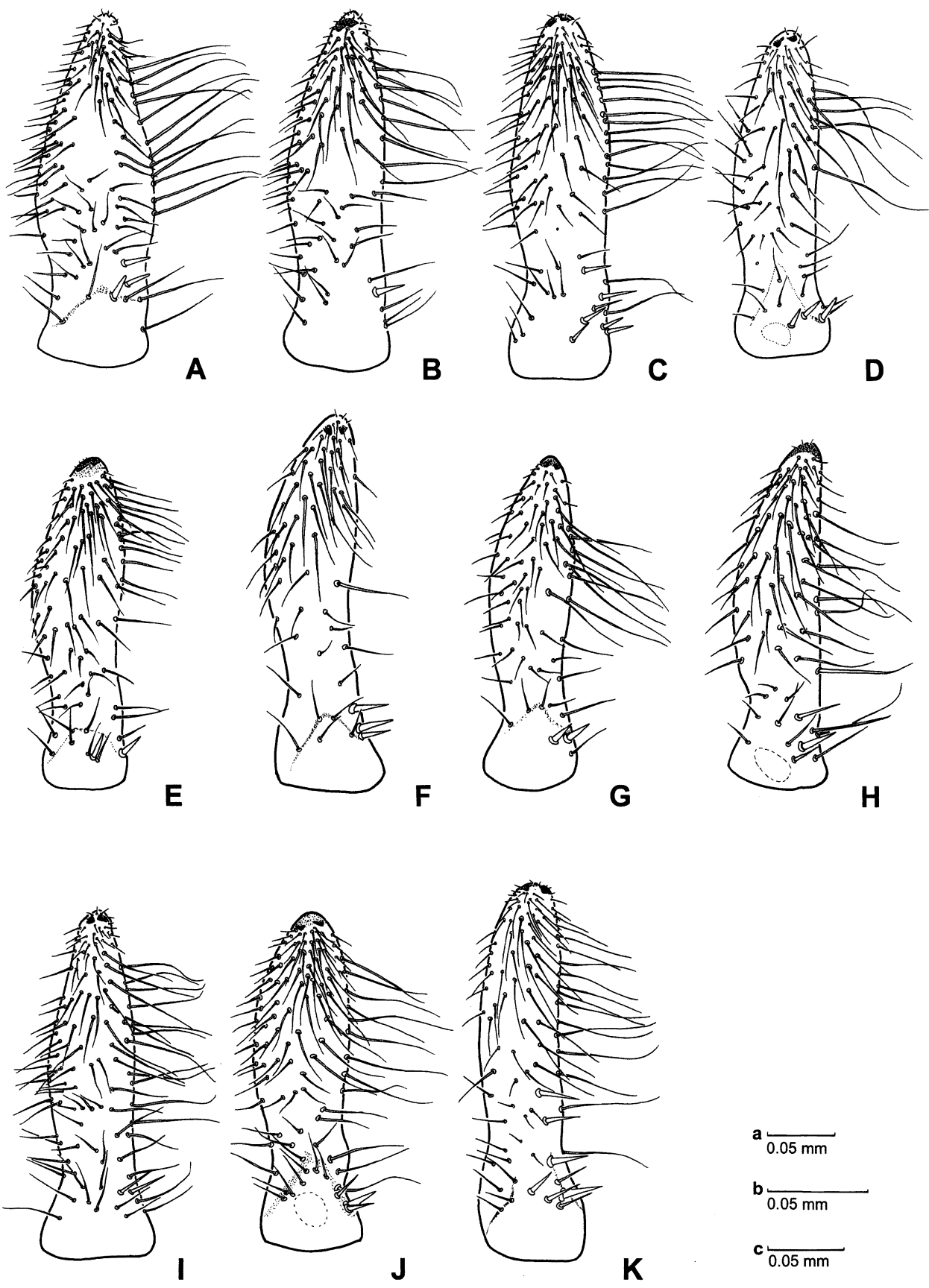

FIG. 25. Paurocephala spp.: (A) P. dayak; (B) P. oceanica; (C) P. marginata; (D) P. palawanensis; (E) P. trematos; (F) P. macrochaetis; (G) P. sauteri; (H) P. boehmeriae; (I) P. setifera; (J) P. polaszeki; (K) P. muta. (A-K) Paramere, inner surface. Scale a, A-E, G, I; b, F; c, H, J, K. 

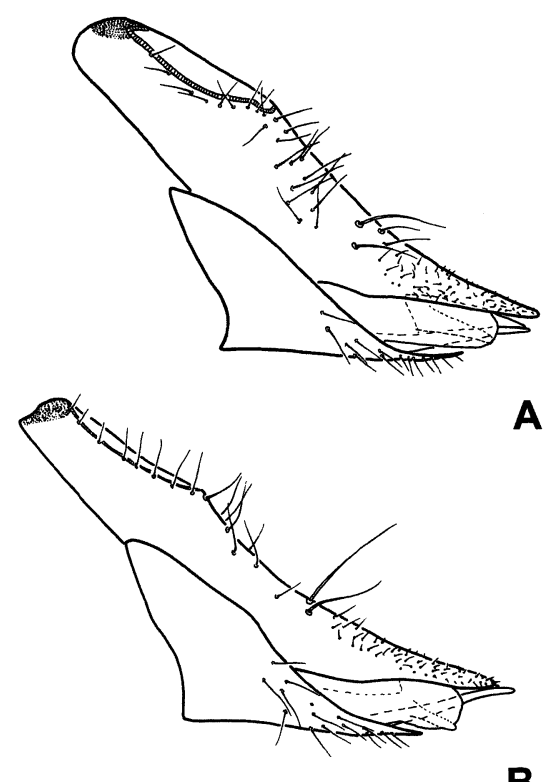

A

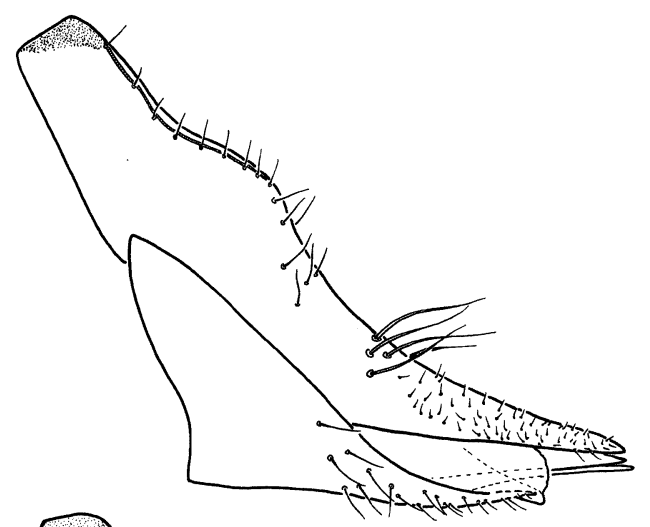

B
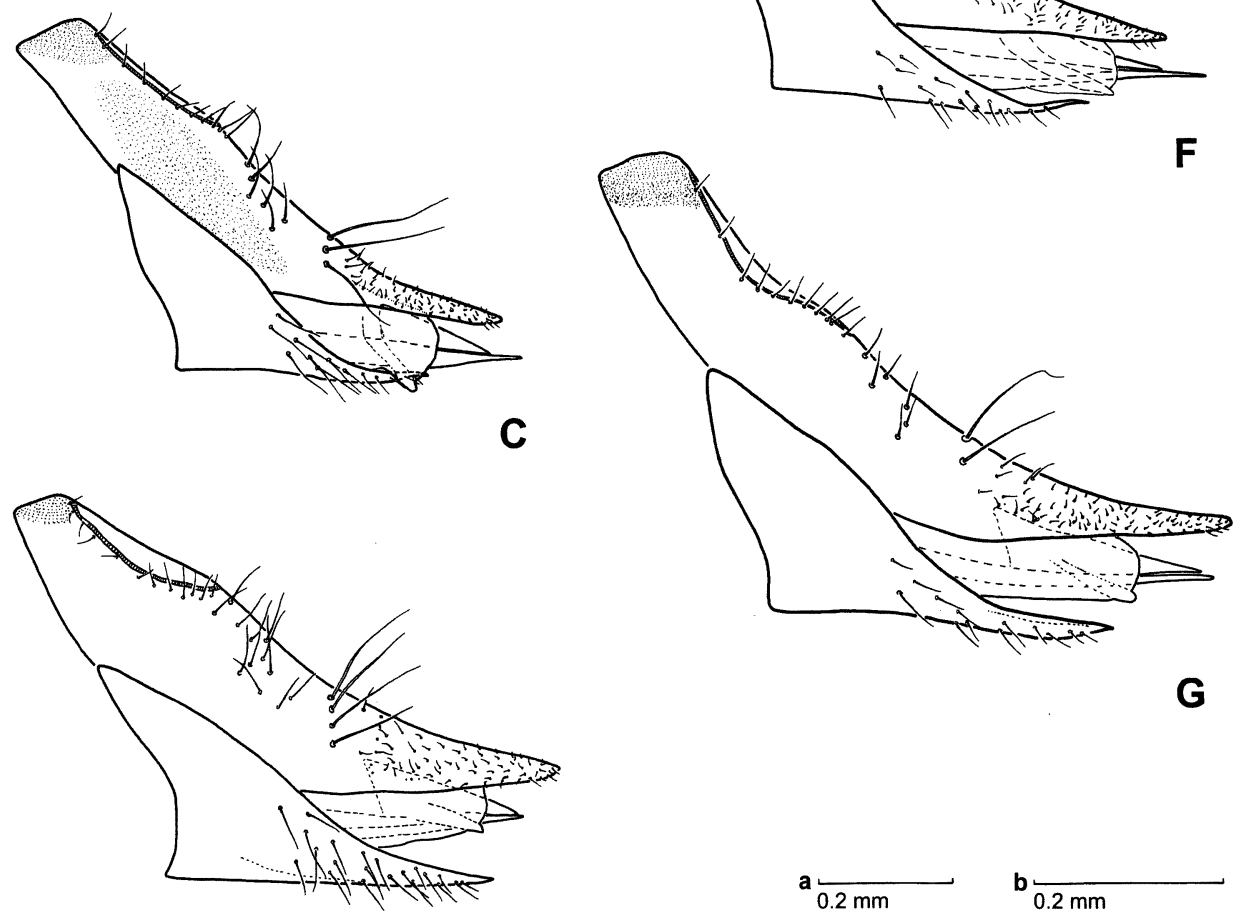

G

D

Fig. 26. Paurocephala spp.: (A) P. dayak; (B) P. oceanica; (C) P. marginata; (D) P. palawanensis; (E) P. trematos; (F) P. macrochaetis; (G) P. sauteri. (A-G) Female genitalia, lateral view. Scale bar: a, A-D; b, E-G. 

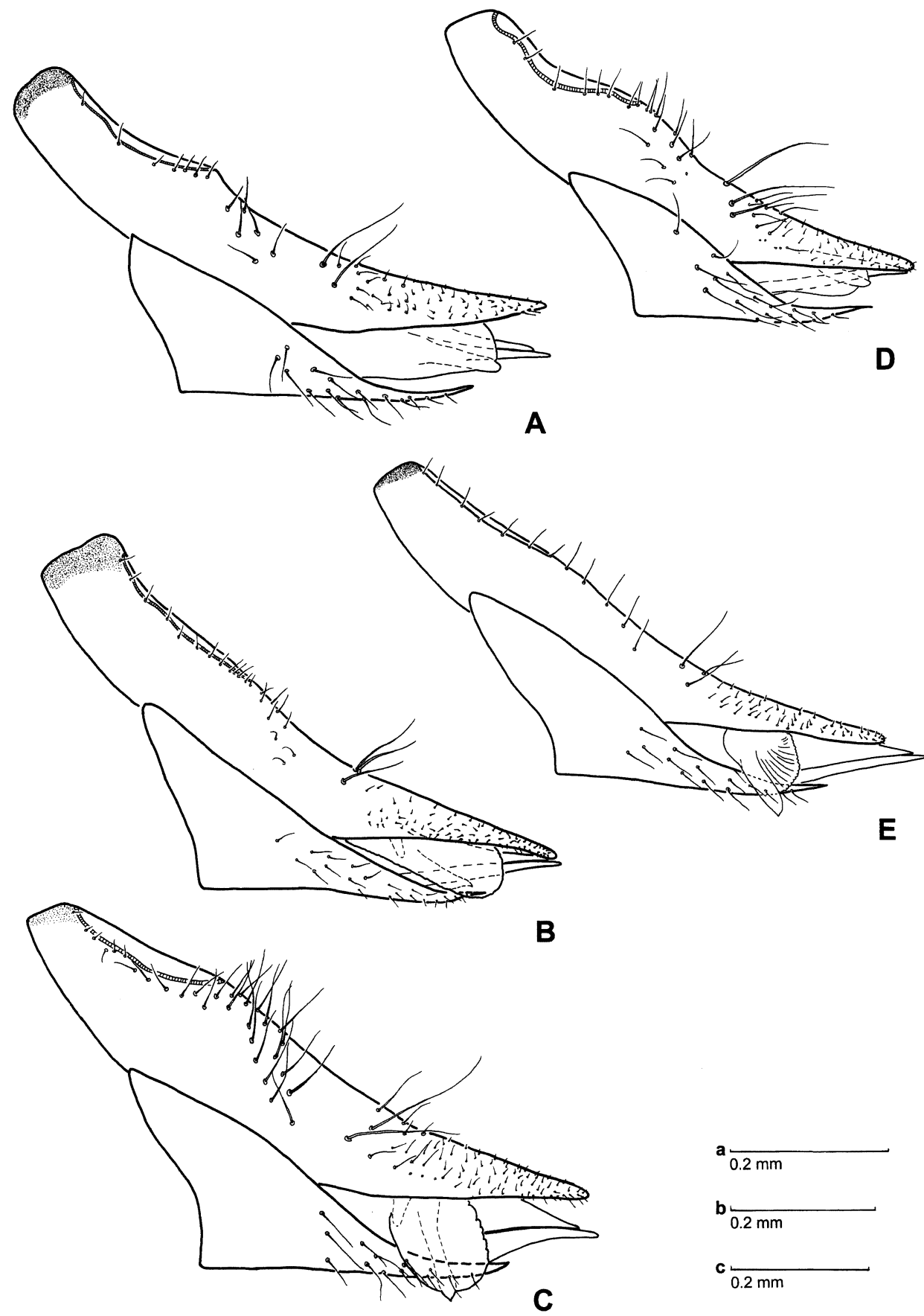

FIG. 27. Paurocephala spp.: (A) P. boehmeriae; (B) P. stigmaticalis; (C) P. setifera; (D) P. polaszeki; (E) P. muta. (A-F) Female genitalia, lateral view. Scale bar: a, A, D; b, B; c, C, E. 


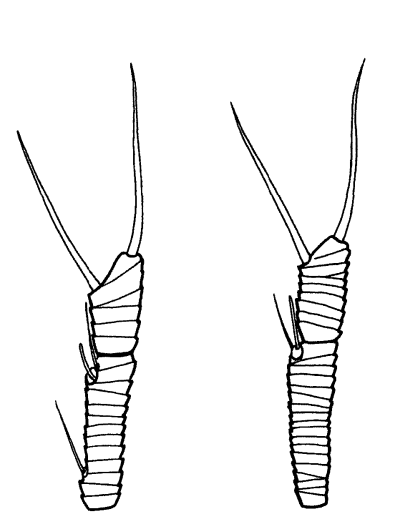

A

B
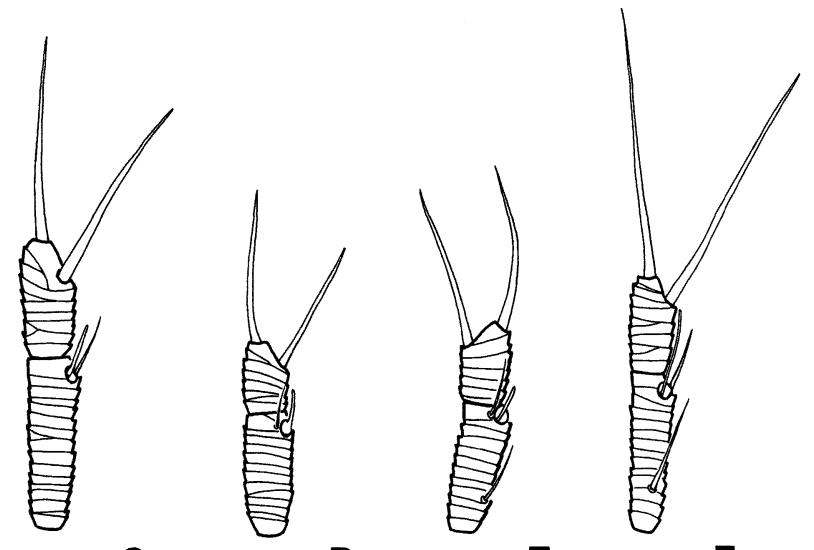

C

D

$\mathbf{E}$

$\mathbf{F}$
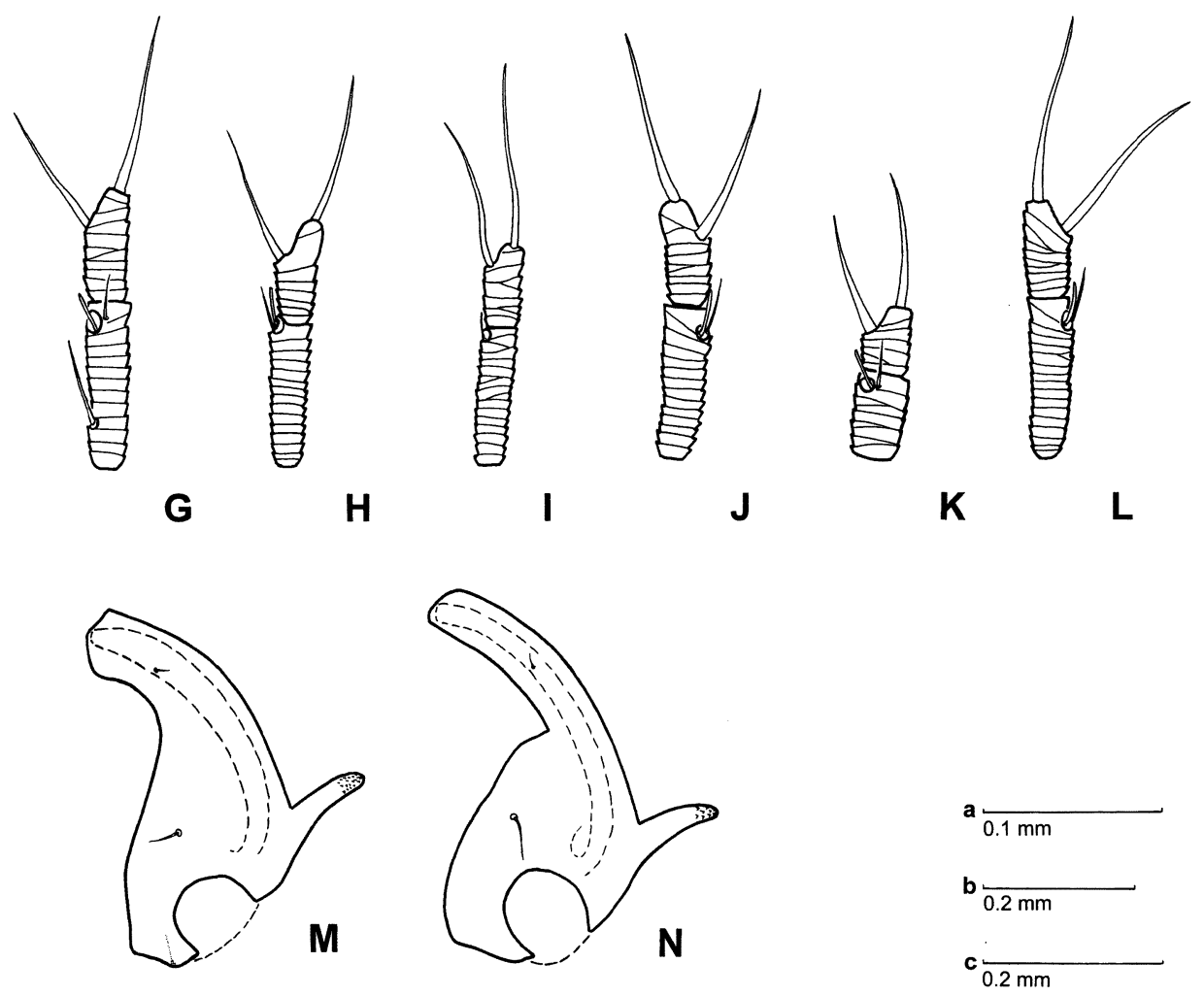

FIG. 28. Paurocephala spp.: (A) P. dayak; (B) P. oceanica; (C) P. marginata; (D) P. palawanensis; (E) P. trematos; (F) P. macrochaetis; (G) P. sauteri; (H, M) P. boehmeriae; (I) P. stigmaticalis; (J) P. setifera; (K, N) P. polaszeki; (L) P. muta. (A-L) Antennal segments 9 and 10; $(\mathrm{M}, \mathrm{N})$ metacoxa. Scale bar: a, A-L; b, M; c, N. 

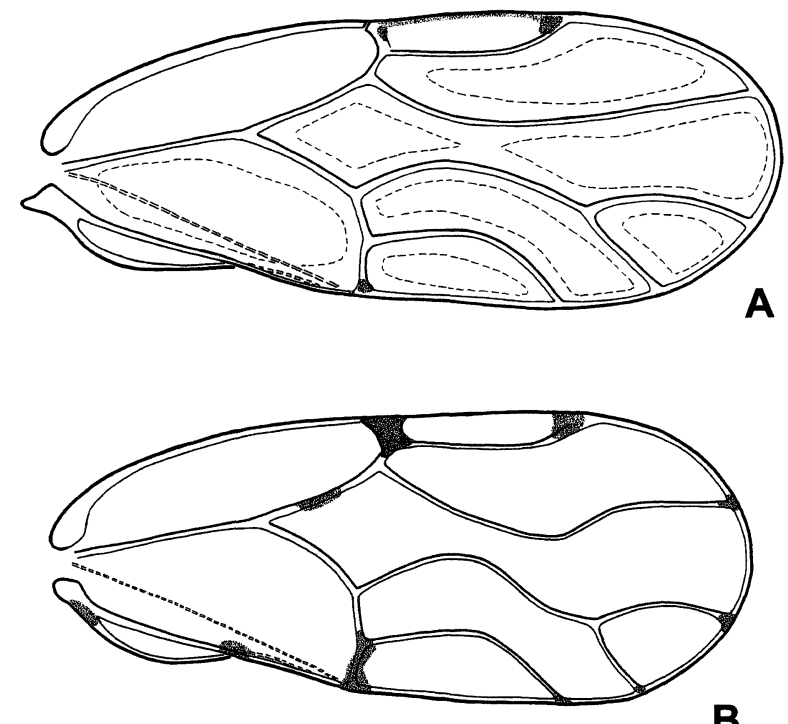

B
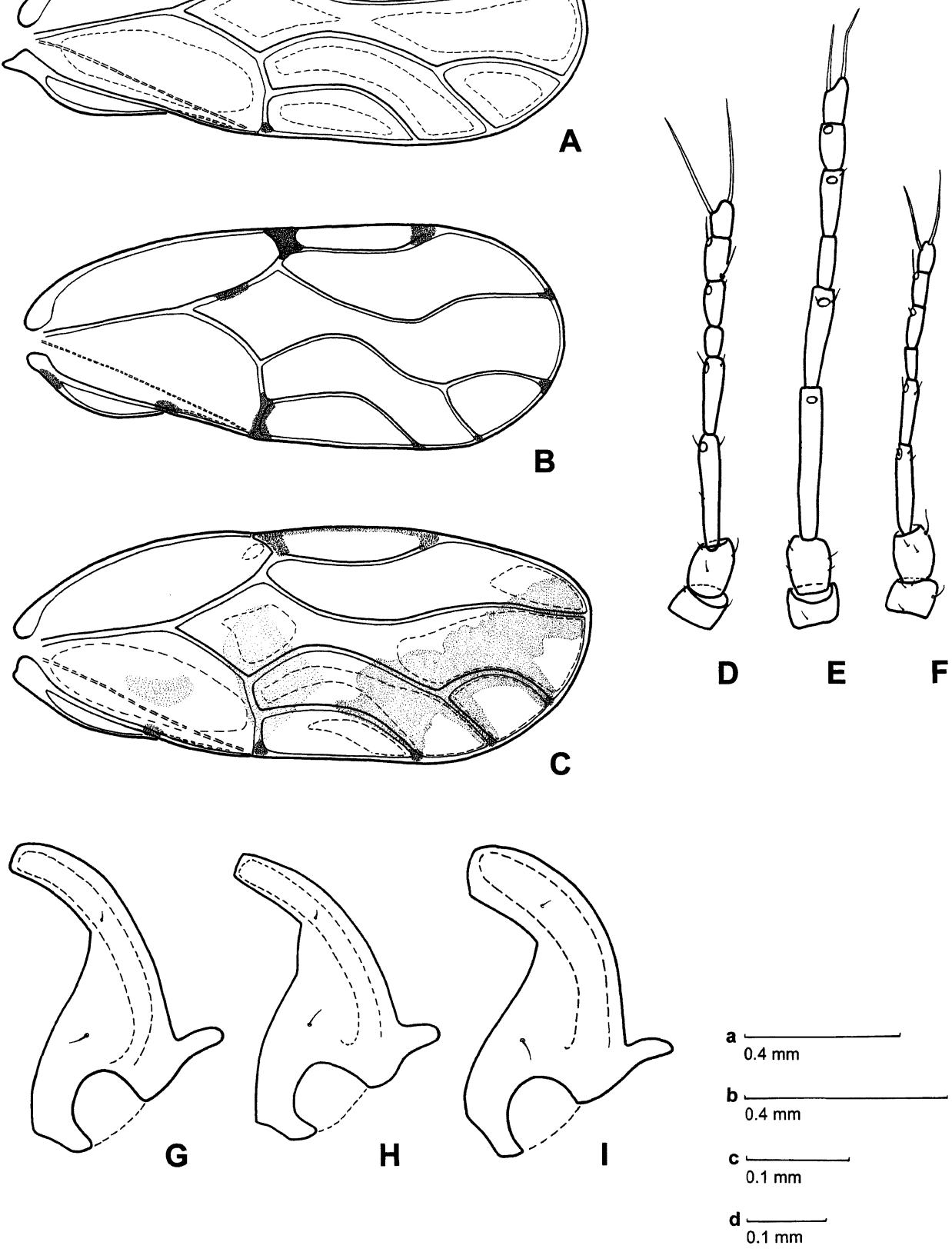

Fig. 29. Paurocephala spp.: (A, D, G) P. lii; (B, E, H) P. mathuri; (C, F, I) P. stephaniella. (A-C) Forewing; (D-F) antenna; (G, H) metacoxa. Scale bar: a, A, B; b, C; c, D-F, I; d, G, H. 


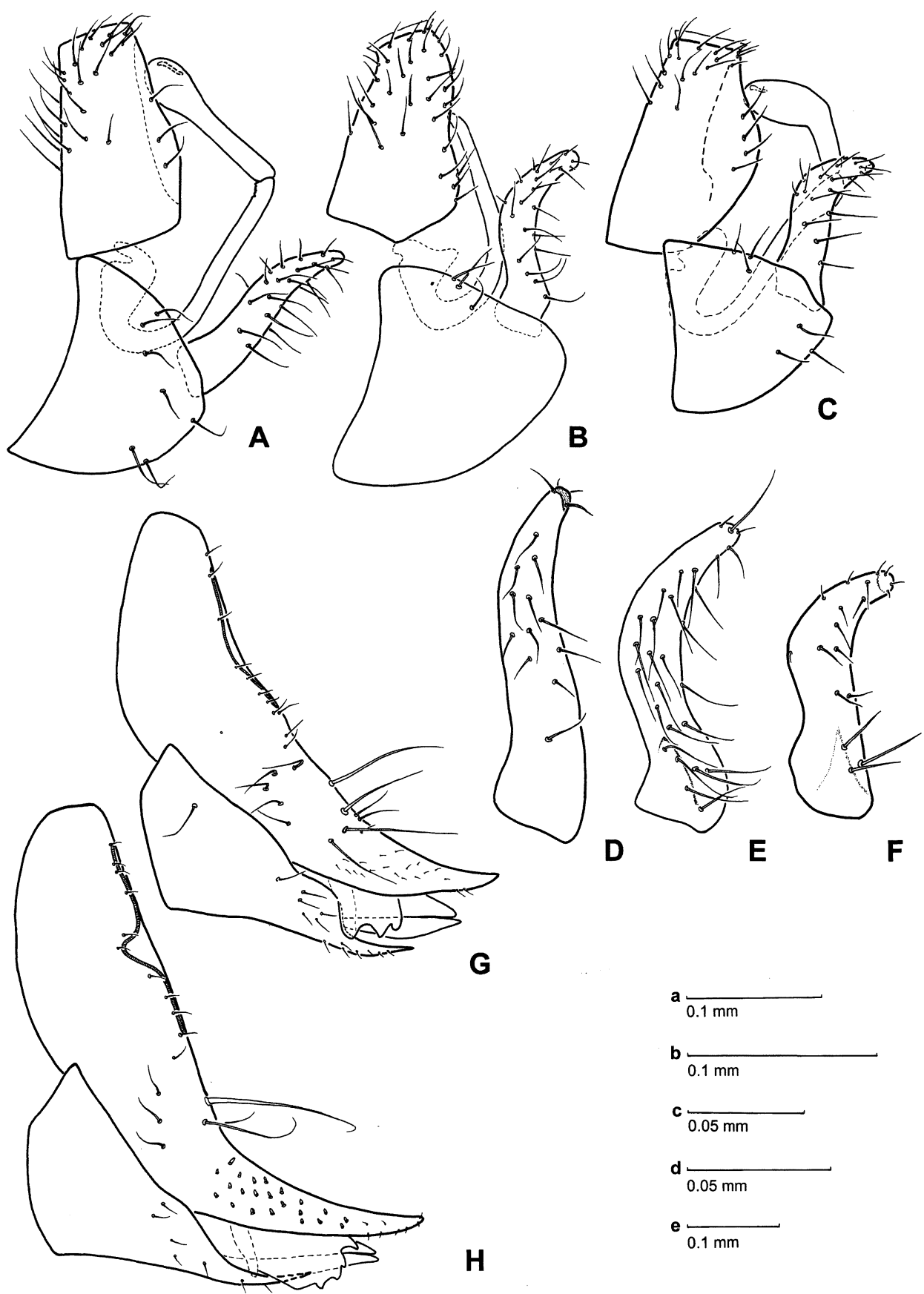

FIG. 30. Paurocephala spp.: (A, D, G) P. lii; (B, E, H) P. mathuri; (C, F) P. stephaniella. (A-C) Male genitalia, lateral view; $(D-F)$ paramere, inner surface; $(G, H)$ female genitalia, lateral view. Scale bar: a, A, B; b, C; c, D, E; d, F; e, G, H. 

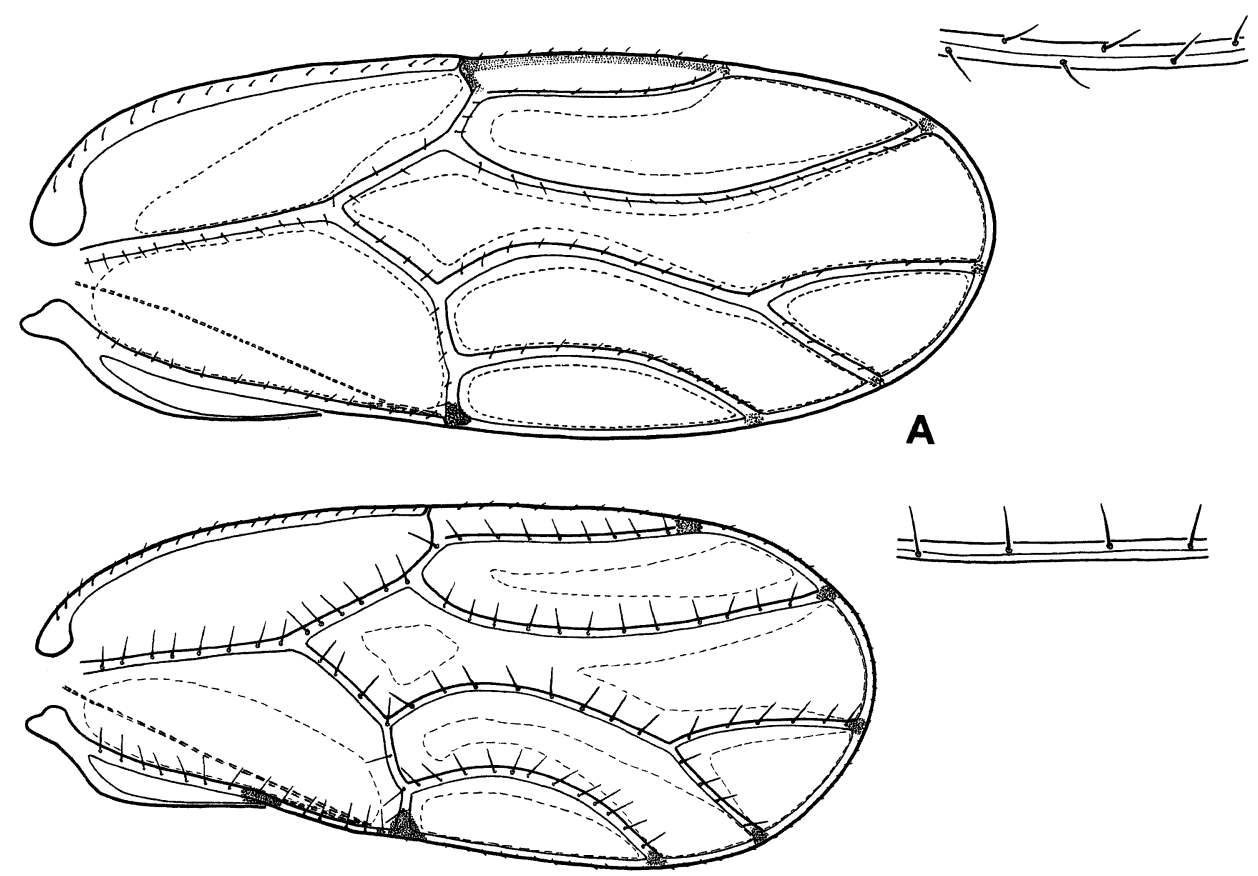

B
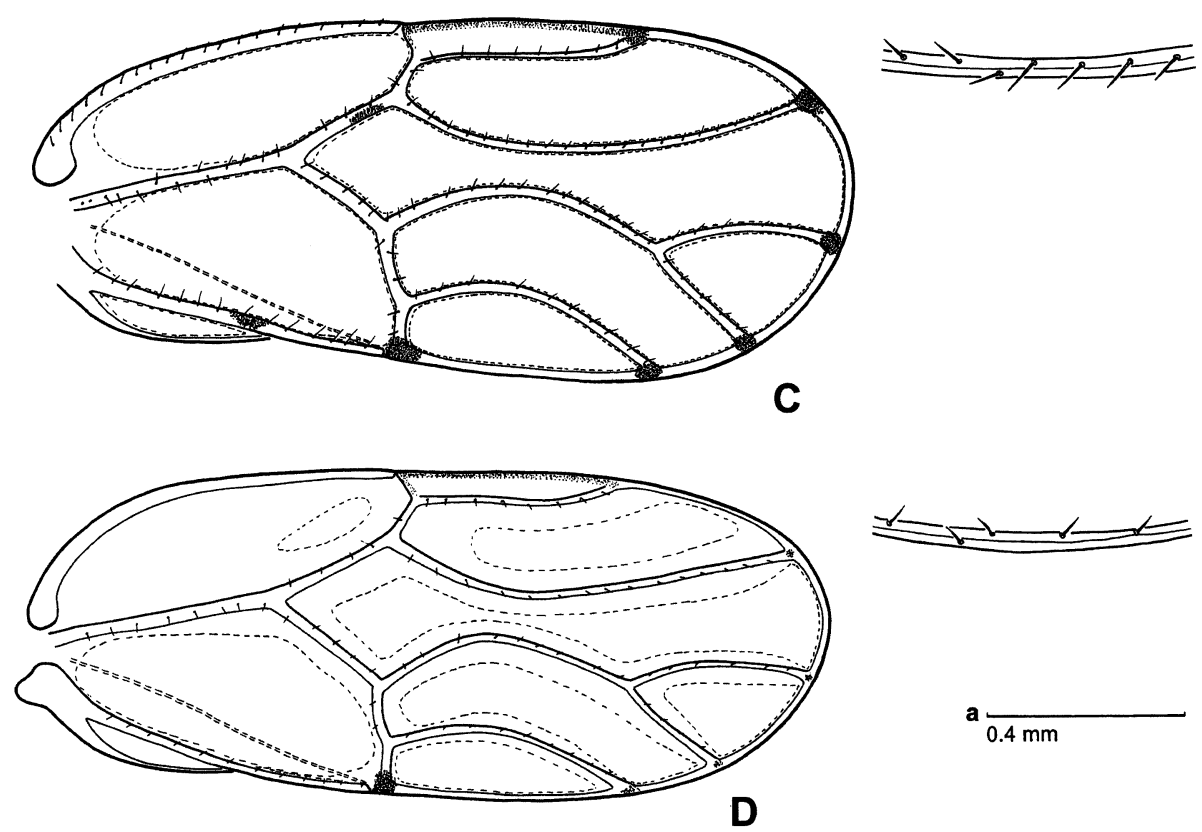

FIG. 31. Paurocephala spp.: (A) P. brevicephala; (B) P. martini; (C) P. robusta; (D) P. sulawesiana. (A-D) Forewing (including detail of middle part of Rs vein). Scale bar: a, A-D. 

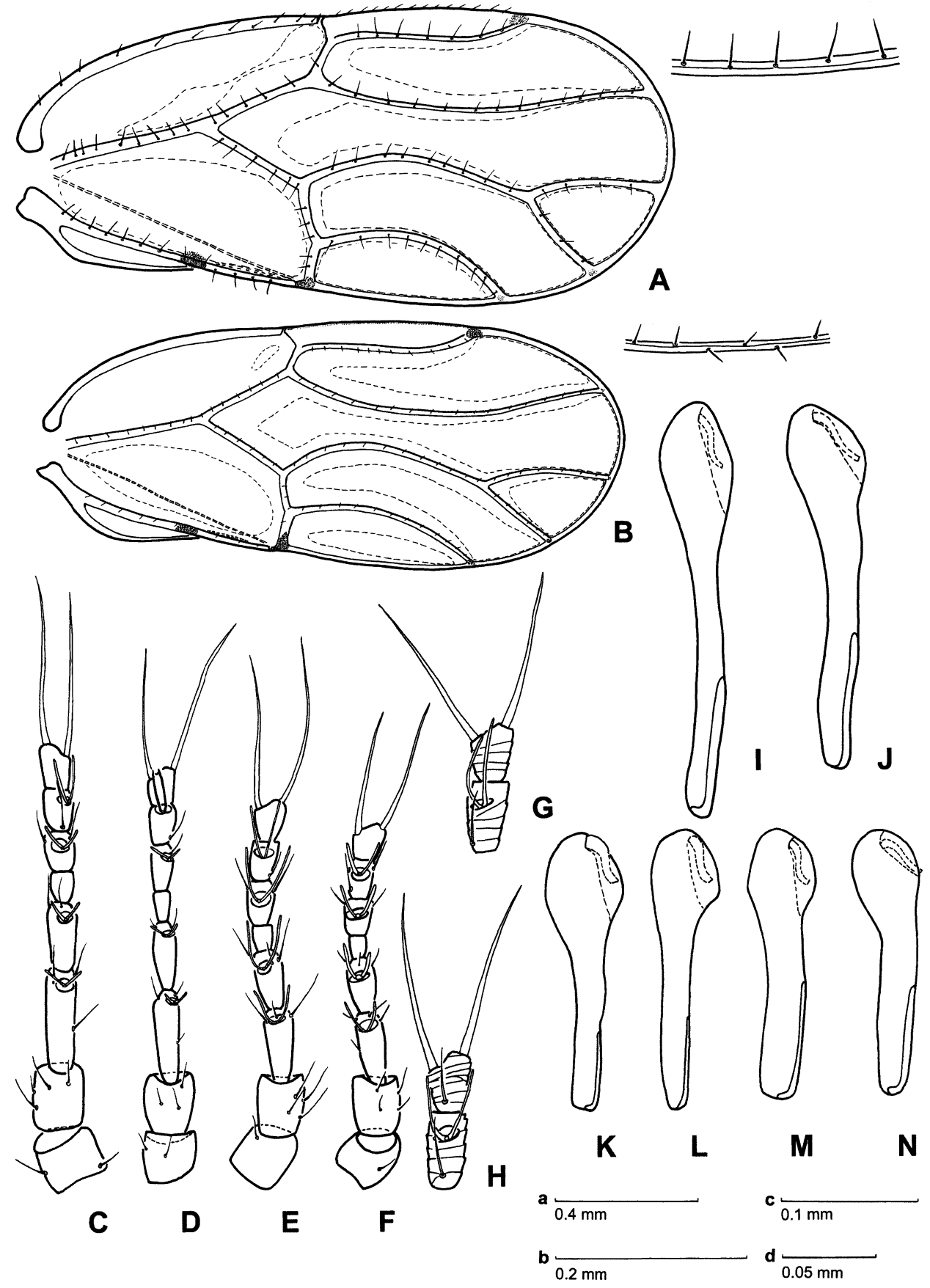

FIG. 32. Paurocephala spp.: (A, C, I) P. ambigua; (B, D, J) P. pterospermi; (E, K) P. martini; (F, L) P. robusta; $(\mathrm{G}, \mathrm{M})$ P. sulawesiana; $(\mathrm{H}, \mathrm{N}) P$. brevicephala. (A, B) Forewing (including detail of middle part of Rs vein); (C-F) antenna; $(\mathrm{G}, \mathrm{H})$ antennal segments 7 and 8; (I-N) distal segment of aedeagus. Scale bar: a, A, B; b, C-F; c, G, $\mathrm{H} ; \mathrm{d}, \mathrm{I}-\mathrm{N}$. 

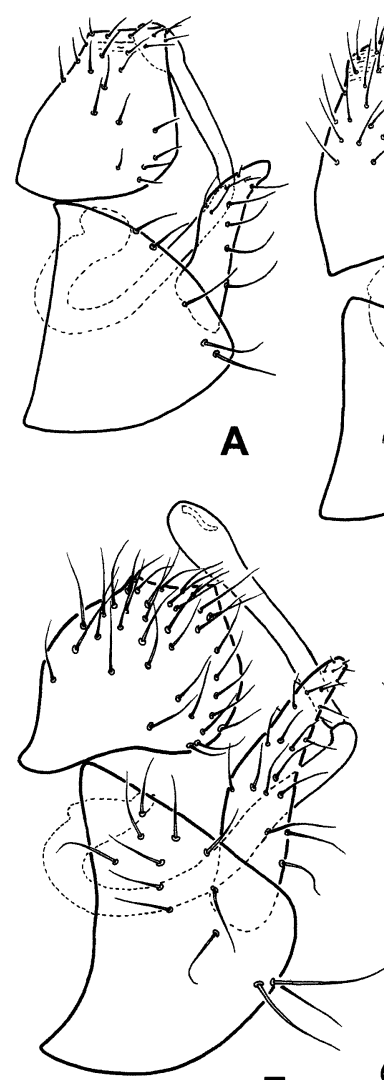

$\mathrm{E}$
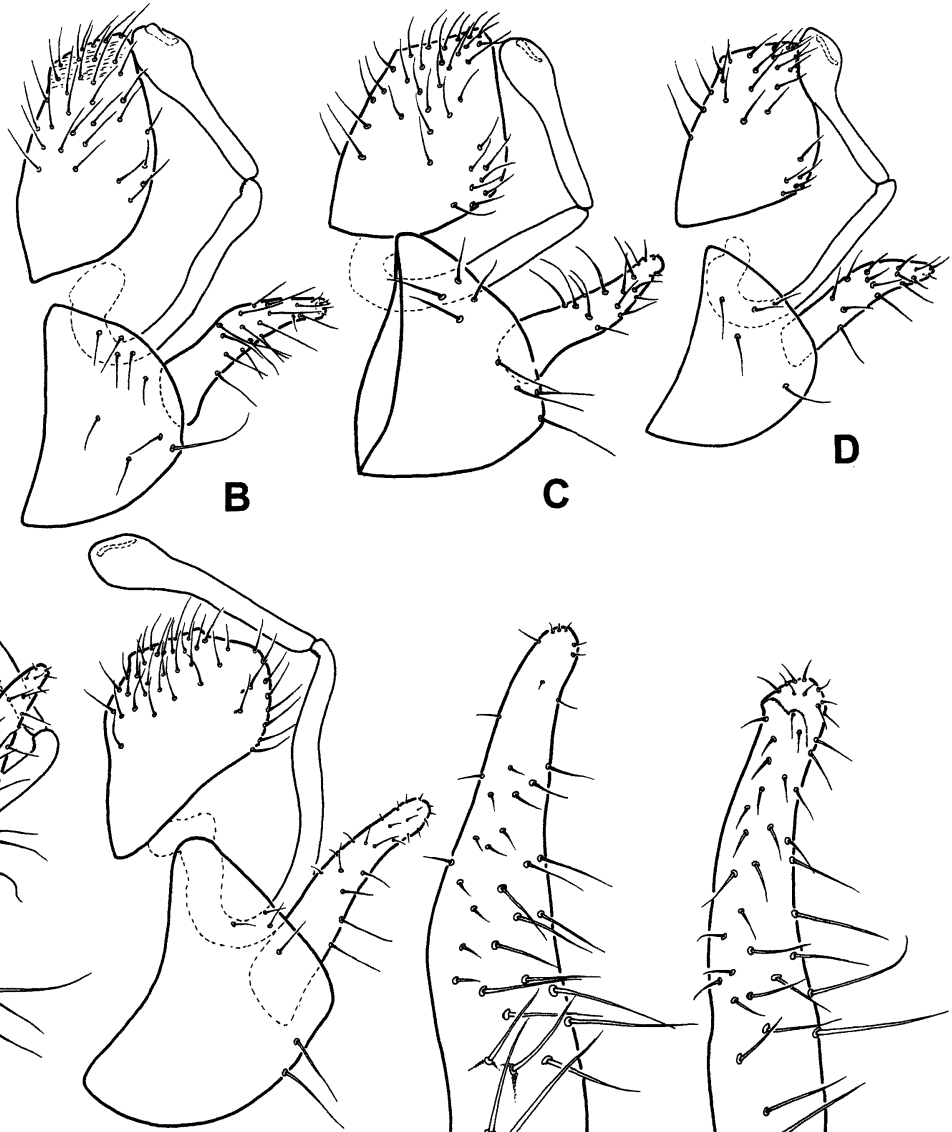

$\mathbf{F}$
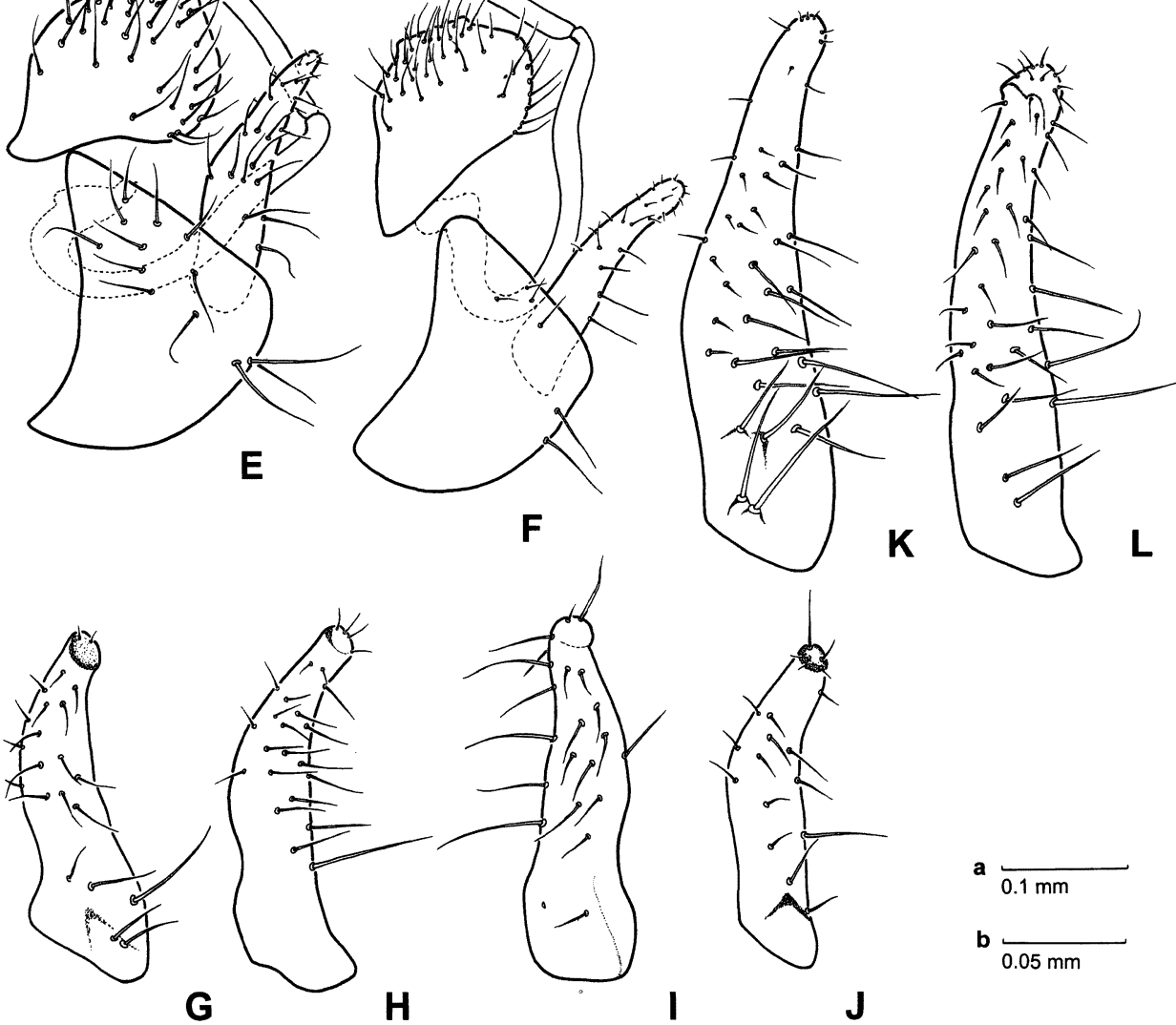

Fig. 33. Paurocephala spp.: (A, G) P. brevicephala; (B, H) P. martini; (C, I) P. robusta; (D, J) P. sulawesiana; (E, K) P. ambigua; (F, L) P. pterospermi. (A-F) Male genitalia, lateral view; (J-L) paramere, inner surface. Scale bar: a, A-F; b, G-L. 


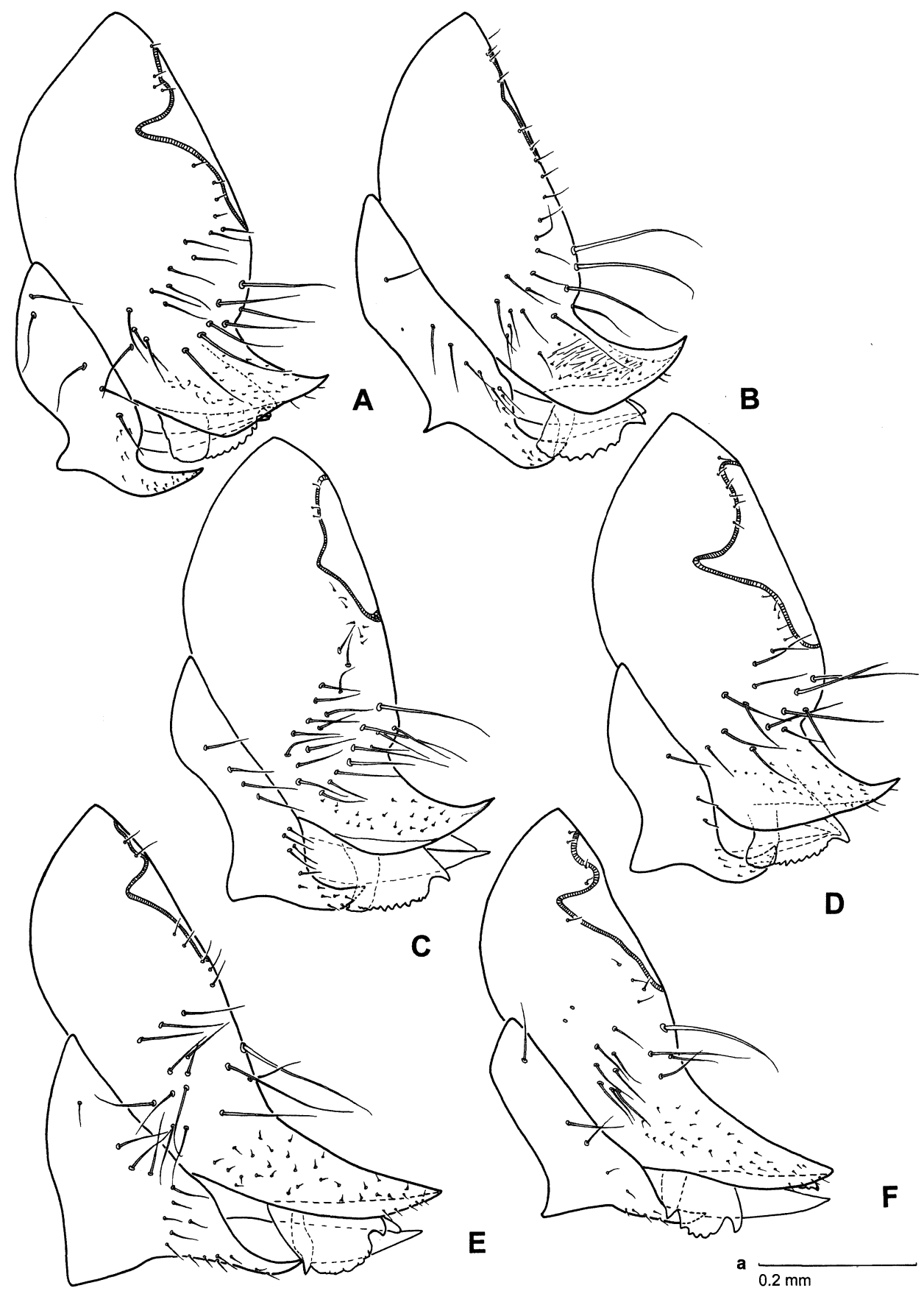

FIG. 34. Paurocephala spp.: (A) P. brevicephala; (B) P. martini; (C) P. robusta; (D) P. sulawesiana; (E) P. ambigua; (F) P. pterospermi. (A-F) Female genitalia, lateral view. Scale bar: a, A-F. 


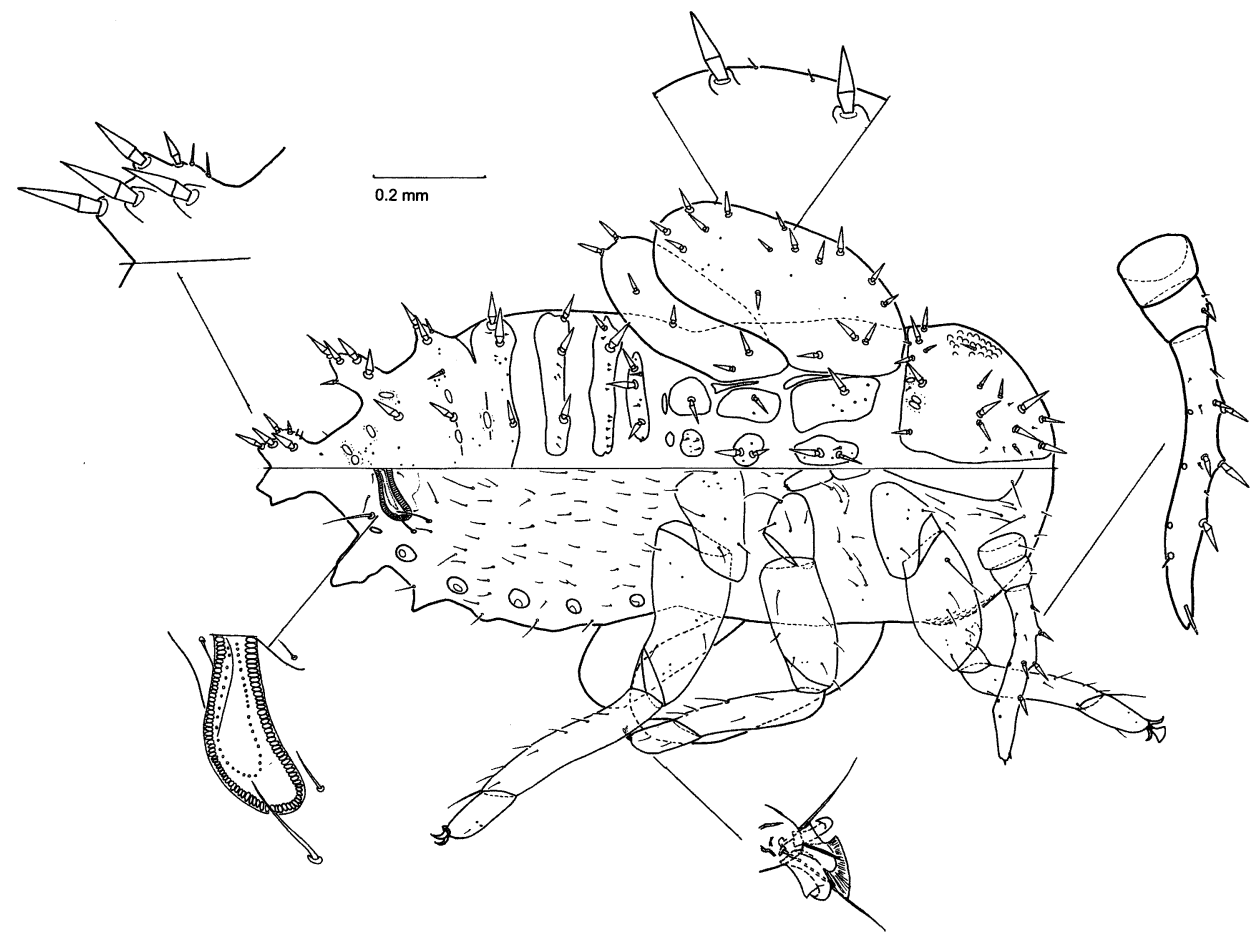

FIG. 35. Fifth instar larva: Paurocephala insolita; left dorsal, right ventral surface.
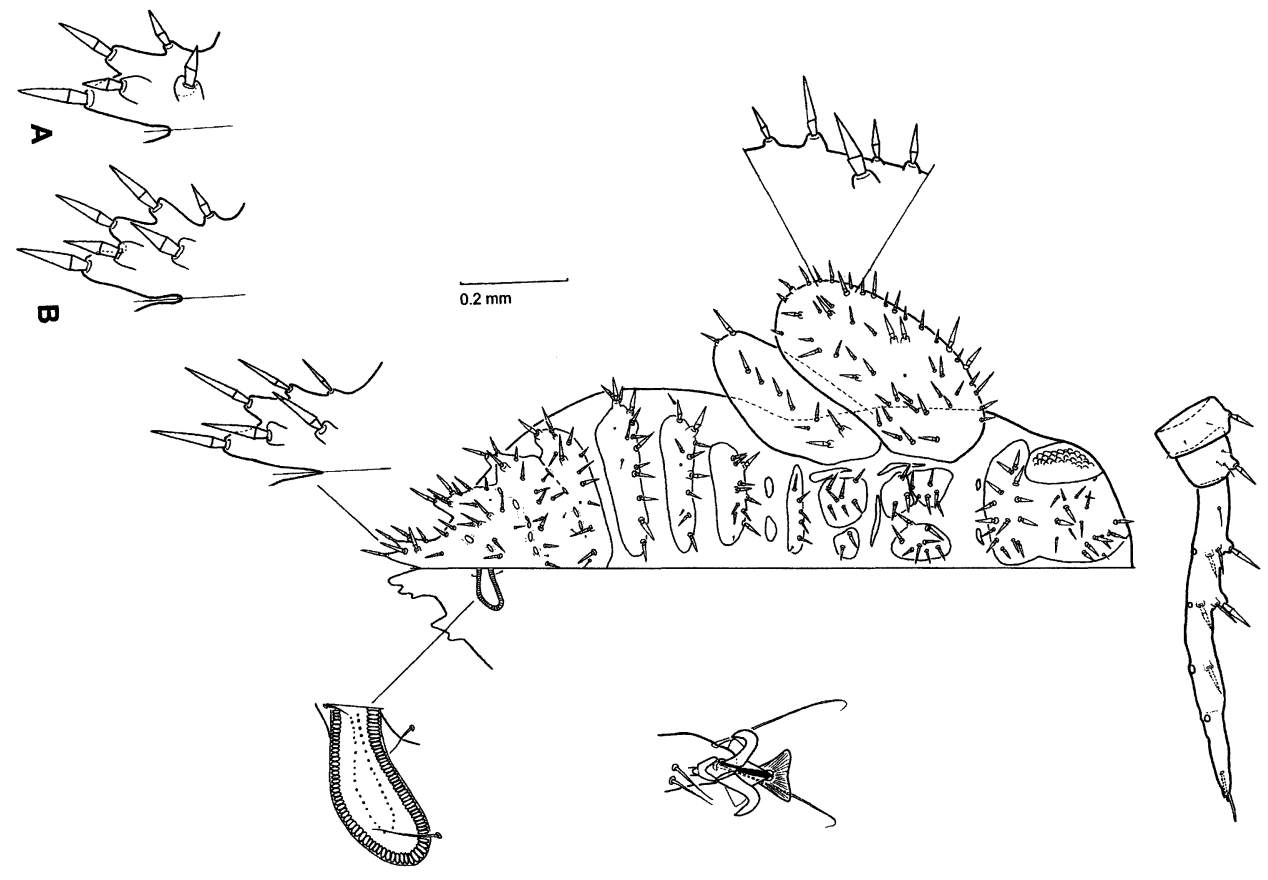

FIG. 36. Fifth instar larva: Paurocephala abutili. (A) P. gossypii; (B) P. urenae. 

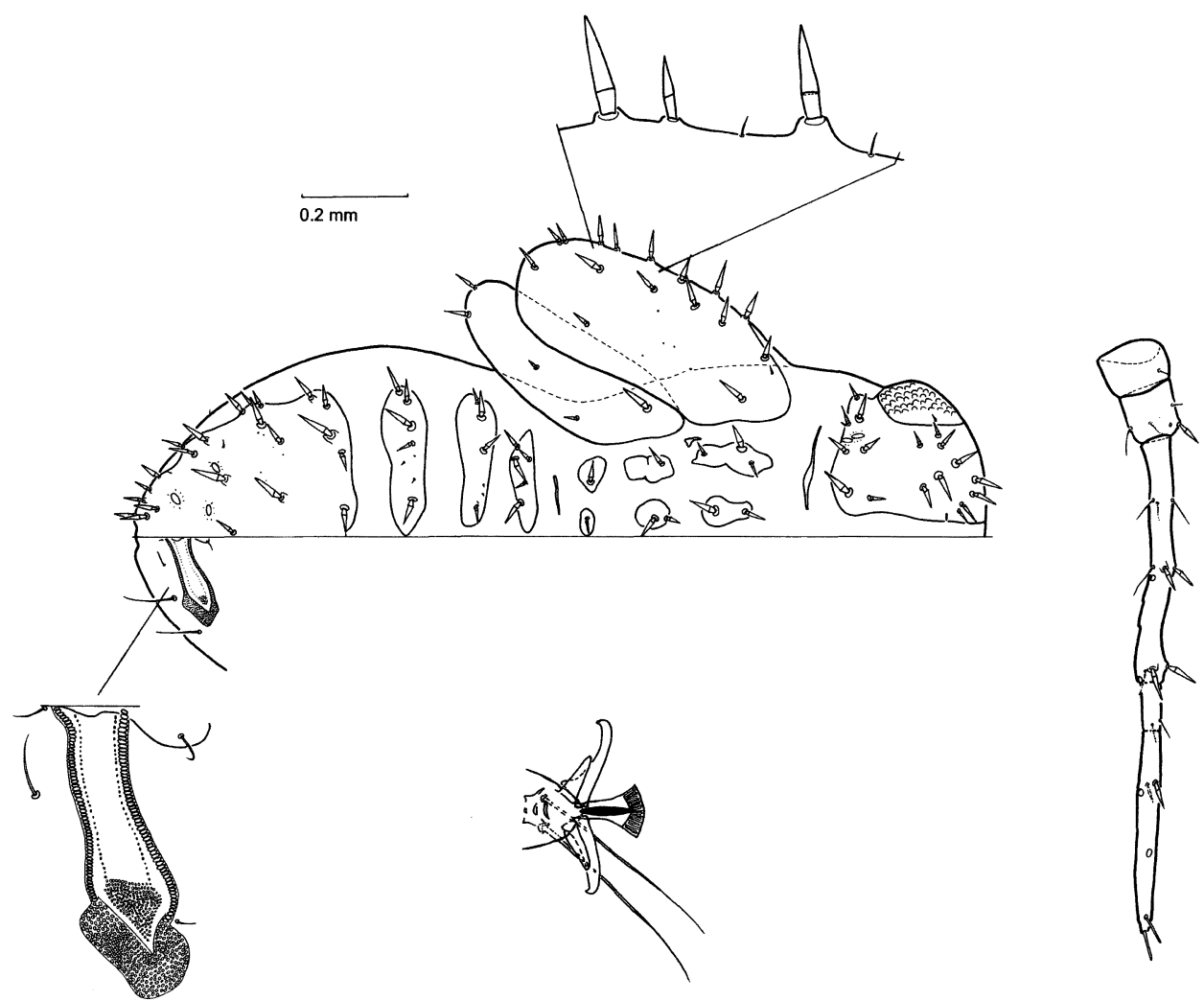

Fig. 37. Fifth instar larva: Paurocephala bifasciata.

Paurocephala Crawford

Paurocephala Crawford, 1914: 293; Hodkinson, 1983: 355; Hodkinson, 1986: 320. Type species: Paurocephala psylloptera Crawford, 1914, by original designation.

Subgenus Thoracocorna Klimaszewski, 1970: 427. Type species: P. chonchaiensis Boselli, 1929, by original designation; synonymized with Paurocephala by Loginova, 1972: 842 .

\section{Description}

Adult. Head down-curved, without genal processes; frons small to moderately sized trapezoidal. Antenna 8-10 segmented, shorter to longer than head width; with each a subapical rhinarium on segments $3,4,6$ and 7, or 3, 5, 7 and 8 , or 4, 6, 8 and 9. Thorax moderately curved dorsally, pronotum relatively long; metascutellum forming a horn-shaped structure. Forewing membrane transparent, often covered by surface spinules, rarely with pattern, pterostigma developed. Metacoxa with short to long meracanthus, straight or curved ventrally (figure 4I, J). Metatibia short to moderately long, without basal spine, weakly widening apically, bearing an incomplete crown of six to eight weakly sclerotized light apical spurs (figure 4F-H). Metabasitarsus without black spurs. Male proctiger simple, tubular, often with distinct lateral plates posteriorly. Male paramere lamellar, often with stout or peg-like setae on inner face. Distal portion of aedeagus relatively short with rounded apical dilatation. Female gentalia cuneate sometimes up-turned apically (figure 3A, B).

Fifth instar larva. Antenna 3-segmented, flagellum often indistinctly subdivided, rarely 5- or 9-segmented; rhinaria formula 3333 (when 3-segmented). Tarsal arolium 


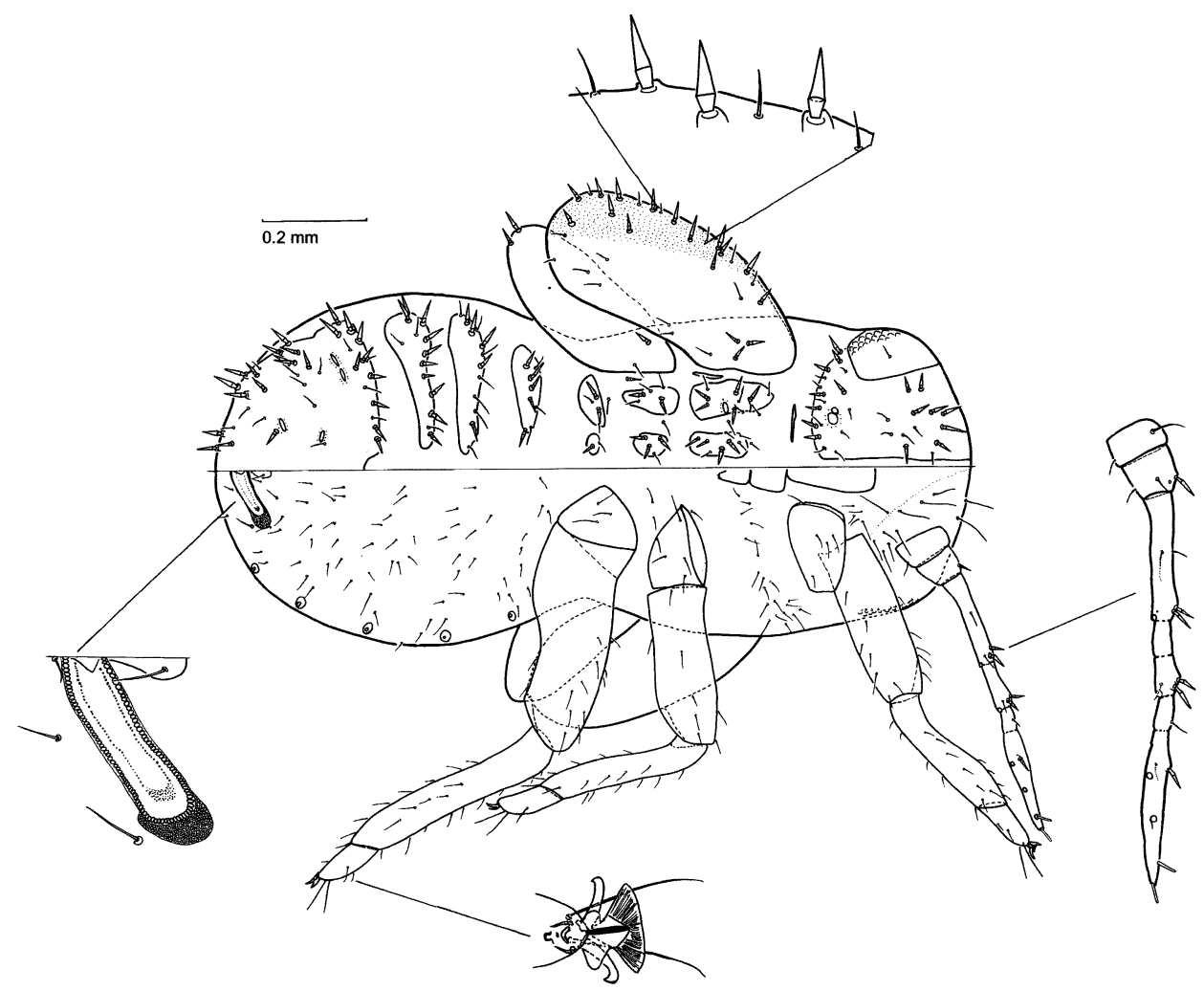

FIG. 38. Fifth instar larva: Paurocephala curvata, left dorsal, right ventral surface.

relatively short triangular to fan-shaped, basally expanded, petiolate with developed unguitractor (figure $4 \mathrm{~K}-\mathrm{N}$ ). Sectasetae present on head, antenna, dorsal sclerites and wing pads. Anus ventral or terminal, circumanal ring simple, sometimes expanded laterally. Additional pore fields lacking.

Comments. The genus is most diverse (42 spp.) in the Indo-Australian region with a relatively small (nine spp.) Afrotropical component. Host records include Malvales (Malvaceae, Sterculiaceae) and Urticales (Moraceae, Urticaceae, Ulmaceae), with a single species on Theales (Clusiaceae). Two Afrotropical species currently included in Paurocephala (bicarinata Pettey and hottentotti Pettey) are excluded here and will be transferred to the Diaphorininae (D. Burckhardt and D. Hollis, personal communication). The four New World Paurocephala spp. and the Asian $P$. grewiae Kandasamy will be transferred to Diclidophlebia Crawford (Burckhardt and Mifsud, in preparation).

\section{Key to Paurocephala}

(Excluding P. minuta Crawford and P. phalaki Mathur, for which material was unavailable or insufficient).

\section{Adults}

1 Metatibia with simple setae laterally, different from apical spurs (figure 4D, G). . . 2

- Metatibia with one or two rows of stout setae laterally, similar to apical spurs

(figure 4C, E, F, H). 


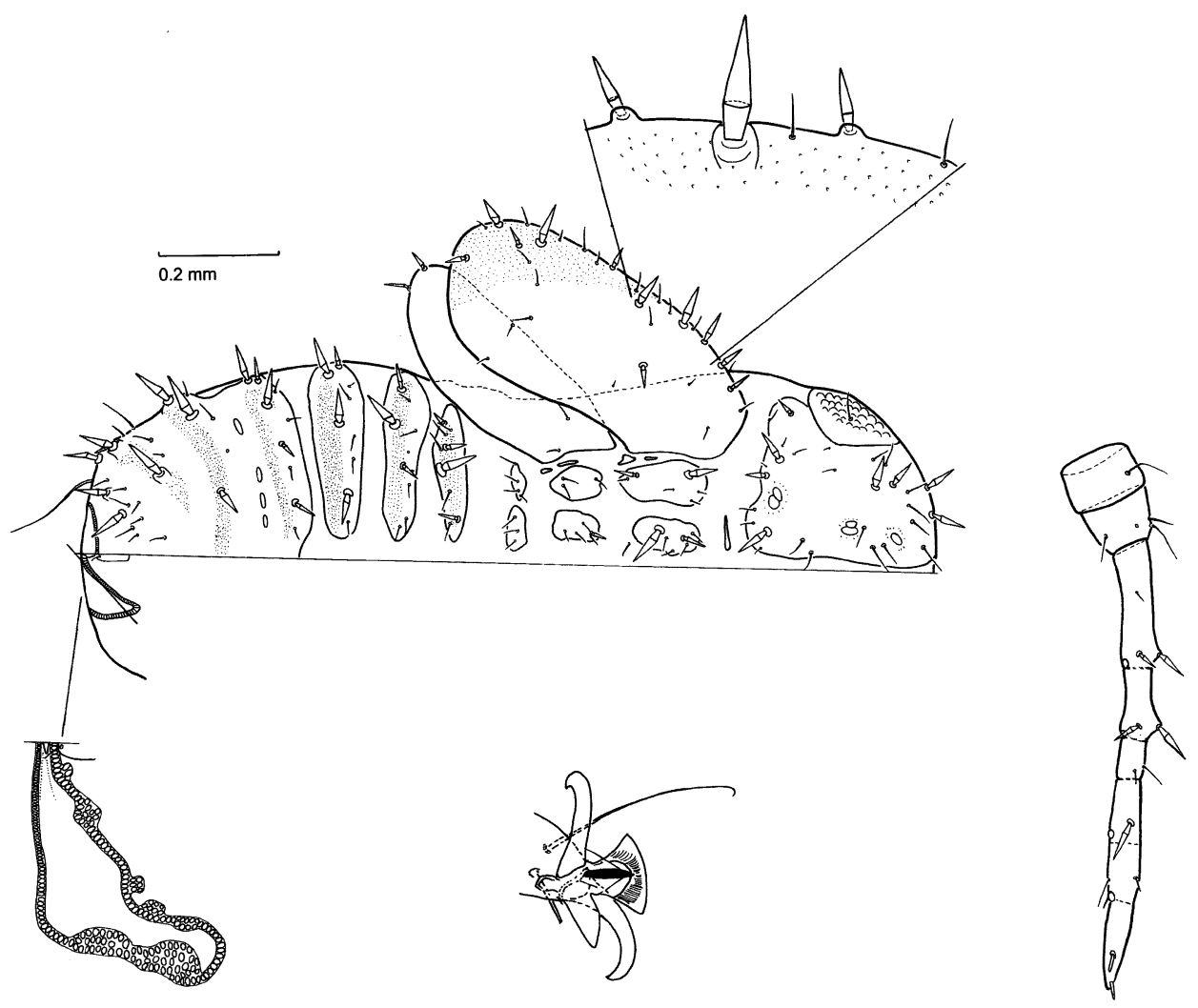

FIG. 39. Fifth instar larva: Paurocephala artocarpae.

2 First four visible abdominal tergites with a group of stout setae laterally (figure 5I).

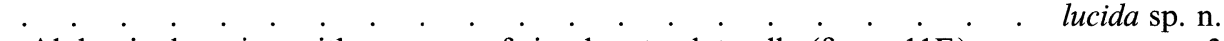

- Abdominal tergites with one row of simple setae laterally (figure 11E).

3 Antennal segment 9 without setae or with one short seta apically. Male paramere without peg-like setae on inner surface (figure 11C, D).

- Antennal segment 9 with two long apical setae as long as or longer than segment. Male paramere with peg-like setae on inner surface (figures 7D, E, 8C, D).

4 Abdominal tergites each with a horn-like structure dorsally (figure 11E). Forewing pointed apically (figure 9E). . . . . . . . . . . . . . brendelli $\mathrm{sp} . \mathrm{n}$

- Abdominal tergites without a horn-like structure dorsally. Forewing rounded apically. 5

5 Forewing dark brown in basal half of pterostigma and base of vein A (figure 9D). Antennal rhinaria with bifid setae. Male paramere rounded apically. . lienhardi $\mathrm{sp} . \mathrm{n}$.

- Forewing brown at base and apex of pterostigma, end of veins to forewing margin, towards middle of veins $\mathrm{R}+\mathrm{M}+\mathrm{Cu}_{1}, \mathrm{R}, \mathrm{M}+\mathrm{Cu}_{1}$ and $\mathrm{A}$. Antennal rhinaria without bifid setae. Male paramere pointed apically.

insolita sp. $\mathrm{n}$.

$6 \mathrm{PT}<0.5$ RL. . . . . . . . . . . . . . . . . . . . 7

- PT>0.5 RL. . . . . . . . . . . . . . . . . . . . . . . . 8

7 Body coloration dark brown. Surface spinules present in cell $\mathrm{c}+\mathrm{sc}$. AL/HW $>1.28$. . .

- Body coloration yellow. Surface spinules absent in cell $\dot{\mathrm{c}}+\dot{\mathrm{sc}} \dot{\mathrm{AL}} / \dot{\mathrm{HW}}<\dot{1.24}$ hollisi sp. $\mathrm{n}$. 


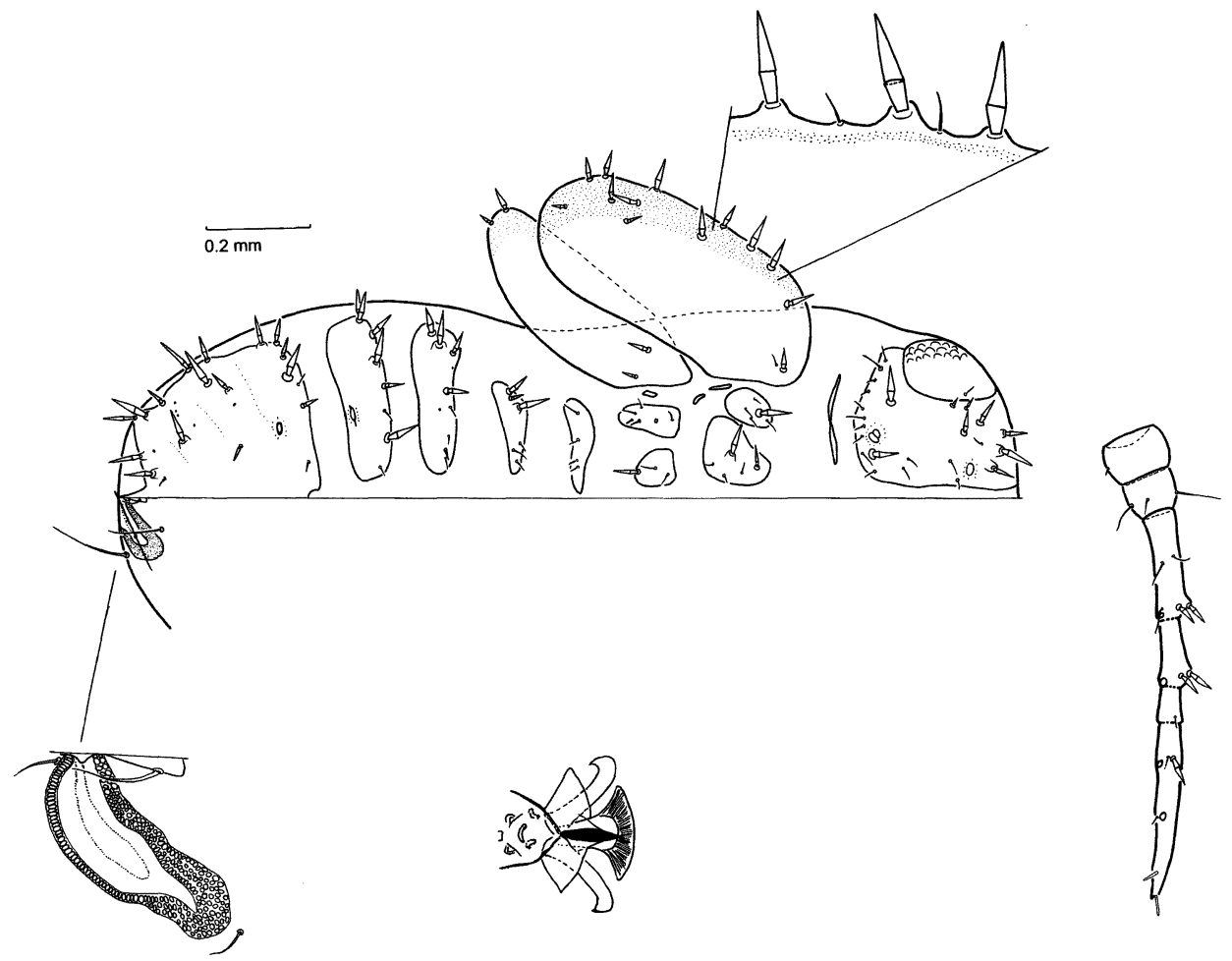

FIG. 40. Fifth instar larva: Paurocephala javanica.

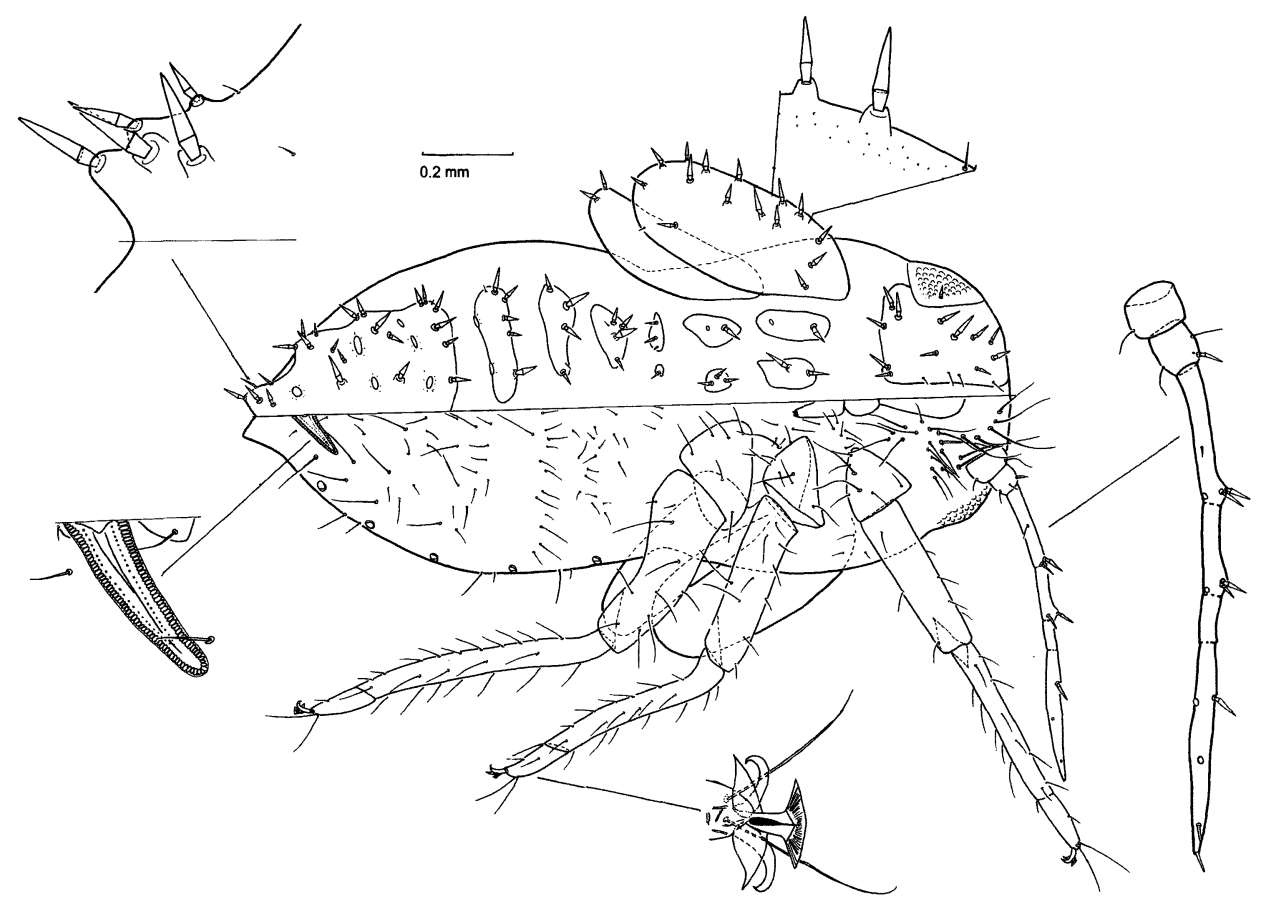

FIG. 41. Fifth instar larva: Paurocephala psylloptera, left dorsal, right ventral surface. 


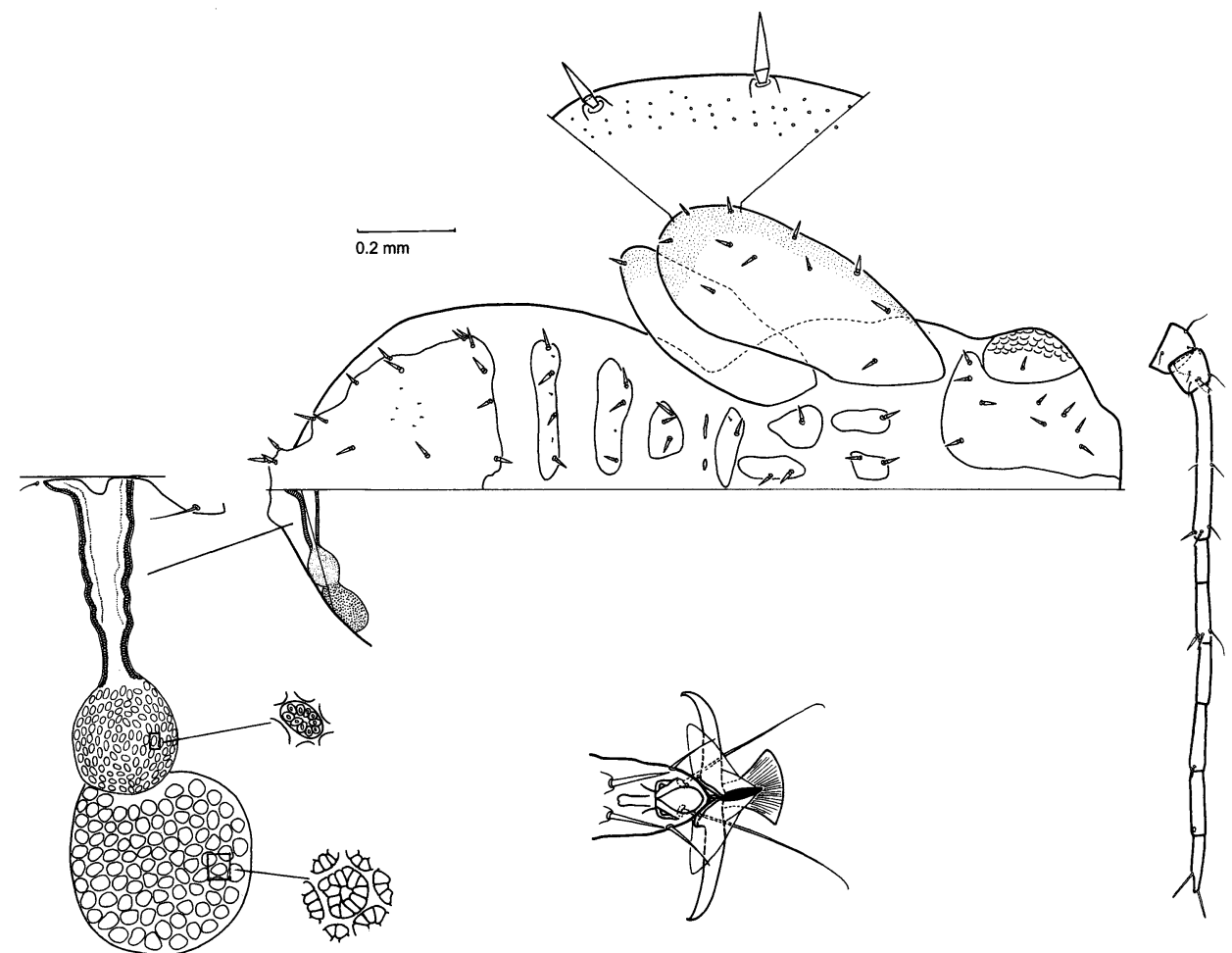

Fig. 42. Fifth instar larva: Paurocephala maculipennis.
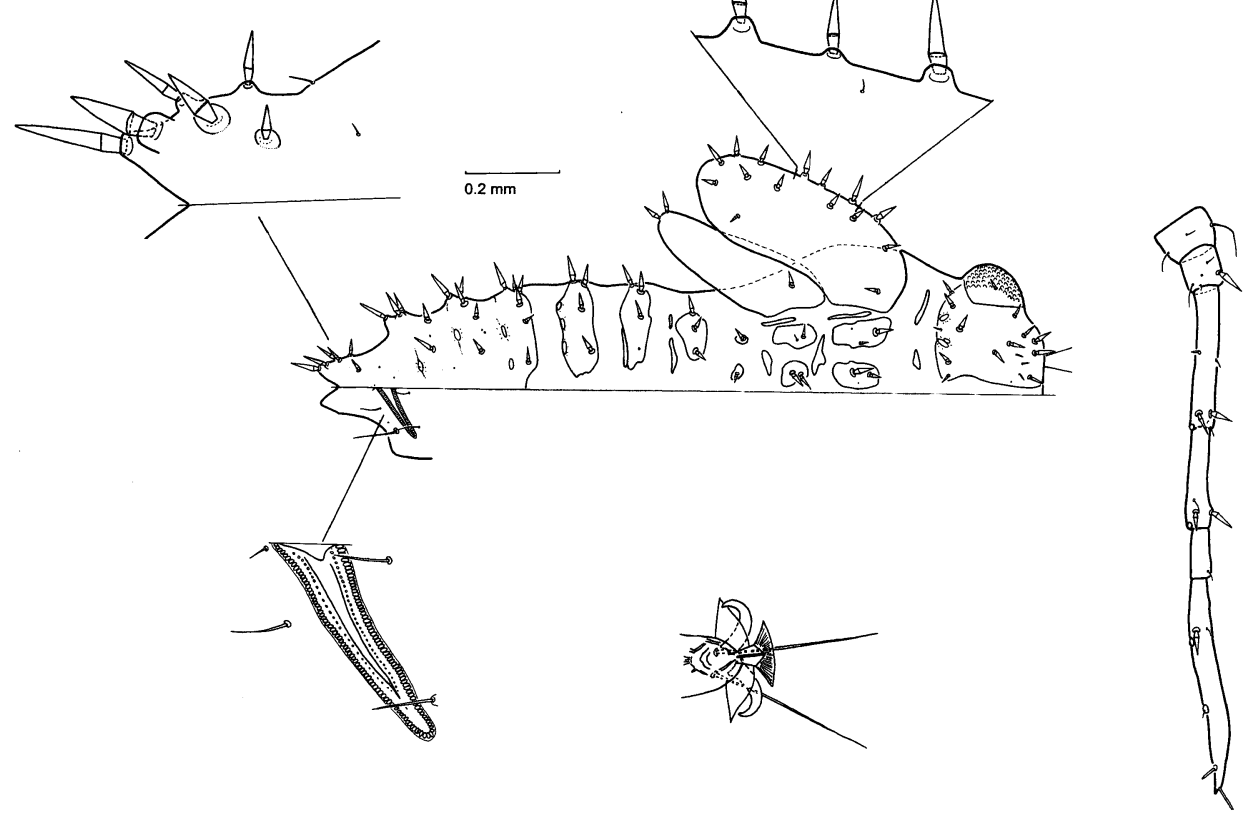

FIG. 43. Fifth instar larva: Paurocephala boehmeriae. 

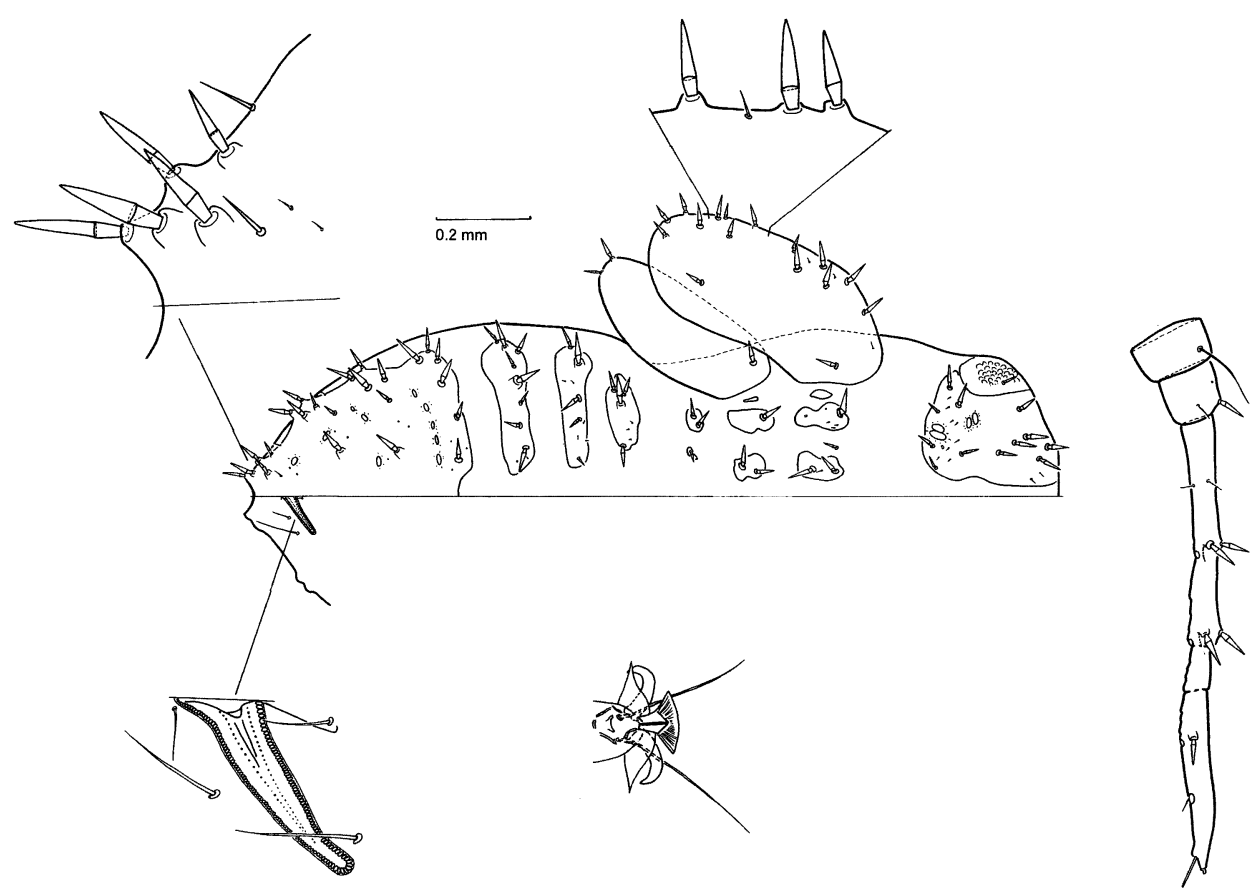

FIG. 44. Fifth instar larva: Paurocephala setifera.

$8 \mathrm{WL} / \mathrm{WW}<2.34, \mathrm{~d} / \mathrm{e}<3.0$. Male proctiger with small rounded posterior plate

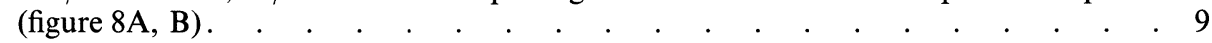

- WL/WW $>2.38, \mathrm{~d} / \mathrm{e}>3.3$. Male proctiger with elongate posterior plate. . . . 10

$9 \mathrm{a} / \mathrm{b}<1.2$. Male paramere with one or two peg-like setae in basal third of inner surface. Female proctiger with straight circumanal ring. . . . . . . . boxisp. $\mathrm{n}$.

$-a / b>1.4$. Male paramere with four peg-like setae in basal third of inner surface. Female proctiger with cruciform circumanal ring. . . . . . . . medleri sp. n.

10 Antennal segments five and seven as long as wide. $\mathrm{AL} / \mathrm{HW}<0.9, \mathrm{ML} / \mathrm{HW}<1.0$.

. . . . . . . . . . . . . . . . . . . . gossypii Russell

- Antennal segments five and seven longer than wide. $\mathrm{AL} / \mathrm{HW}>1.0, \mathrm{ML} / \mathrm{HW}>1.0$. . 11

11 Head, pronotum, mesothoracic praescutum and scutum dark brown. Female proctiger with inner and outer rows of circumanal ring having equal number of pores (figure 5A).. . . . . . . . . . . . . . . . . . . . abutili sp. n.

- Head, pronotum, mesothoracic praescutum and scutum yellow to orange. Female proctiger with inner row of circumanal ring with a reduced number of pores than outer one (figure 5C) . . . . . . . . . . . . . . . urenae Russell

12 Abdominal tergites each with a horn-like structure dorsally. . . . . . . . 13

- Abdominal tergites without horn like-structures dorsally. . . . . . . . . 15

13 Metascutellar horn pointed and elongate laterally. Terminal seta longer than flagellum

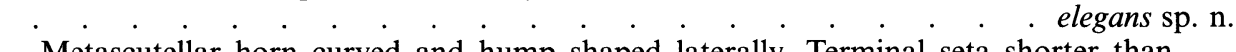

- Metascutellar horn curved and hump shaped laterally. Terminal seta shorter than flagellum . . . . . . . . . . . . . . . . . . . . . 14

14 Antenna 9-segmented. . . . . . . . . . . . . kleinhofiae Uichanco

- Antenna 10-segmented. . . . . . . . . . . . . . russellae Mathur

15 Head short, vertex length to width ratio $>2.8$. Antenna 10 -segmented. AL/HW $>1.1$.

Female genitalia with lateral valvulae membraneous (figure 3A, C). . . . . . 16 


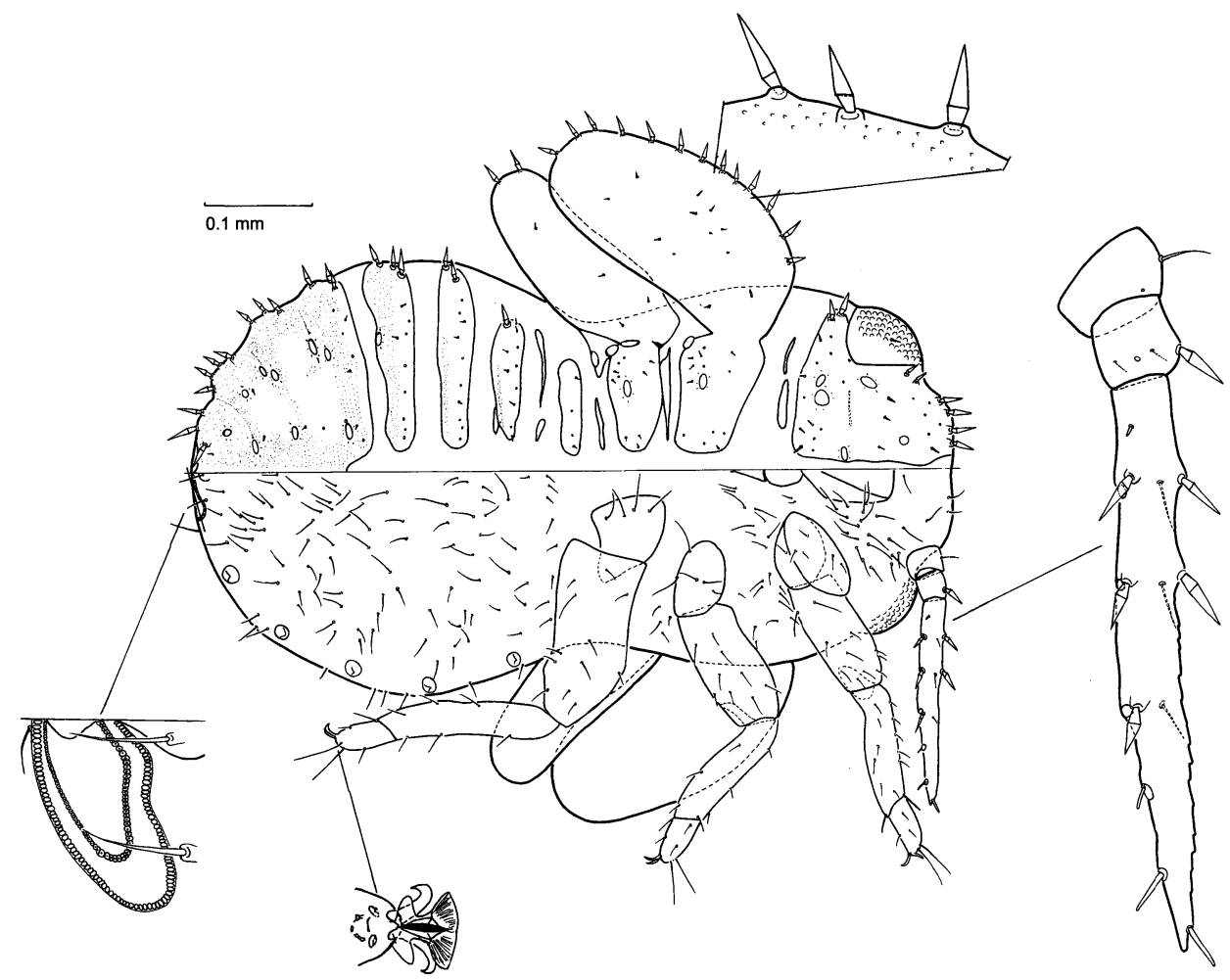

FIG. 45. Fifth instar larva: Paurocephala robusta, left dorsal, right ventral surface.

- Head long, vertex length to width ratio < 2.6. Antenna 8-segmented. AL/HW $<0.9$. Female genitalia with lateral valvulae sclerotized and serrated ventrally (figure $3 \mathrm{~B}, \mathrm{D}$ ).

16 Forewing with two brown patterns: from costal break to anal break and towards apex (from apical third of $\mathrm{Rs}$ vein towards apical half of $\mathrm{Cu}_{1 \mathrm{a}}$ vein) (figure 12A, B).

- Character combination different.

$17 \mathrm{AL} / \mathrm{HW}<1.4$. Cells in apical half of forewing densely covered by surface spinules (figure 12B). Setae on Rs vein longer than distance between two adjacent ones.

. . . . . . . . . . . . . . . chonchaiensis Boselli

- AL/HW > 1.5. Cells in apical half of forewing sparsely covered by surface spinules (figure 12A). Setae on Rs vein as long as or shorter than distance between two adjacent ones .

. bifasciata Kuwayama

18 First visible abdominal tergite without a branching finger-like structure laterally. Male proctiger without lateral plates (figure 14A-F). . . . . . . . . . . . 19

- First visible abdominal tergite with a branching finger-like structure extended on a stalk directed anteriorly, laterally. Male proctiger with lateral plates (figure 18A-F). 24

19 Forewing with conspicuous setae on veins; setae on Rs vein as long as or longer than distance between two adjacent ones.

- Forewing with inconspicuous setae on veins; setae on Rs vein shorter than distance between two adjacent ones.

20 Forewing light brown; forewing cells densely covered by surface spinules. Male paramere with two layers of stout setae in basal half (figure 15B). 
- Forewing transparent; forewing cells sparsely covered by surface spinules. Male paramere with one layer of stout setae in basal half (figure 15E). javanica $\mathrm{sp} . \mathrm{n}$.

$21 \mathrm{ML} / \mathrm{HW}>1.3$. Female genitalia long: $\mathrm{FP} / \mathrm{SL}<2, \mathrm{SL}>0.35 \mathrm{~mm}$.

- $\mathrm{ML} / \mathrm{HW}<1.3$. Female genitalia short: $\mathrm{FP} / \mathrm{SL}>2, \mathrm{SL}<0.25 \mathrm{~mm}$.

$22 \mathrm{CL}>0.16 \mathrm{~mm}$. Female proctiger with oval circumanal ring. Male paramere straight posteriorly.

- $\mathrm{CL}<0.13 \mathrm{~mm}$. Female proctiger with cruciform circumanal ring. Male paramere S-shaped posteriorly.

similis $\mathrm{sp} . \mathrm{n}$.

23 Forewing angular apically, with a brown coloration towards apex (figure 13F). PT $>0.5 \mathrm{RL}$. Male paramere broadest basally, tapering straight towards apex, with stout setae on inner surface (figure 15F). wilderi Crawford

- Forewing rounded apically, without a brown coloration at apex. PT $<0.5$ RL. Male paramere broadest in the middle, apical part directed anteriorly, without stout setae on inner surface (figure 15C). curvata sp. $\mathrm{n}$.

$24 \mathrm{Rs}$ vein distinctly curved in the middle (figure $17 \mathrm{~A}, \mathrm{~B}$ ). $\mathrm{RL} / \mathrm{RC}<1.21$. . . . . 25

- Rs vein indistinctly curved in the middle (figure $17 \mathrm{C}-\mathrm{F}$ ). $\mathrm{RL} / \mathrm{RC}>1.23$. . . . . . 26

25 Larger species: $\mathrm{WL}>2.5 \mathrm{~mm}, \mathrm{AL} / \mathrm{HW}>2.2$. Male paramere with stout setae on inner surface confined to basal two-thirds (figure 19B). . . . . . longiantennata $\mathrm{sp} . \mathrm{n}$.

- Smaller species: WL $<2.4 \mathrm{~mm}, \mathrm{AL} / \mathrm{HW}<2.1$. Male paramere with stout setae on inner surface confined to basal half (figure 19A). . . . . maculipennis Uichanco

26 Antennal segment 9 with a long seta in basal third (figure 28A). . . . . . . . . 27

- Antennal segment 9 without a long seta in basal third (figure 28B). . . . . . 34

$27 \mathrm{CL}>0.12 \mathrm{~mm}$, straight laterally (figure $18 \mathrm{H}$ )..$\quad$. . . . . . . . . . . . . . . 28

- CL $<0.11 \mathrm{~mm}$, marginally curved laterally (figure $28 \mathrm{~N}$ ). . . . . . . . . 31

28 Base of pterostigma forming an angle $>90^{\circ}$ to forewing margin (figure 17F). Male paramere without stout setae on inner surface. FP/SL $>2.4 .+$ distincta sp. $\mathrm{n}$.

- Base of pterostigma forming an angle $<90^{\circ}$ to forewing margin (figure 17A-E). Male paramere with stout setae on inner surface. FP/SL $<2.2$.

29 Rs vein with $23-28$ setae. AL/HW $<1.4$. Seta on antennal rhinaria of segments 8 and 9 short (figure $19 \mathrm{H}$ ). WL $<1.6, \mathrm{ML} / \mathrm{HW}<1.3$. . . . . . . . muiri sp. $\mathrm{n}$.

- Rs vein with 14-21 setae. AL/HW $>1.5$. Seta on antennal rhinaria of segments 8 and 9 long (figure 19G). WL $>1.6, \mathrm{ML} / \mathrm{HW}>1.4$.

30 Male paramere straight, tapering towards apex, inner surface with a row of 5-8 stout setae in basal half (figure 19C). $\mathrm{FP} / \mathrm{CL}<3.8 .+. \quad . \quad . \quad . \quad$. psylloptera Crawford

- Male paramere curved posteriorly in apical third, inner surface with a group of 1-3 stout setae at base (figure 19E). FP/CL $>4.0$.

papuana $\mathrm{sp} . \mathrm{n}$.

31 Surface spinules present in all forewing cells, rarely absent in cell $\mathrm{c}+\mathrm{sc}$. . . . . 32

- Surface spinules confined to cells in apical half of forewing: in $\mathrm{cu}_{1}$, apex of $\mathrm{m}_{1}, \mathrm{~m}_{2}$ and rarely in $r_{2}$.

32 Setae on Rs vein as long as or longer than distance between two adjacent ones. Male paramere widest in the middle, with long setae posteriorly (figure 25A). . dayak sp. $\mathrm{n}$.

- Setae on Rs vein shorter than distance between two adjacent ones. Paramere widest in distal third, with short setae posteriorly (figure 25E).

trematos Yang et al.

33 Smaller species: $\mathrm{WL}<1.62 \mathrm{~mm}, \mathrm{ML} / \mathrm{HW}>1.4$. Terminal seta more than three times length of antennal segment 10 (figure $28 \mathrm{~F}$ ) . . . . . . . macrochaetis $\mathrm{sp} . \mathrm{n}$

- Larger species: WL $>1.71 \mathrm{~mm}, \mathrm{ML} / \mathrm{HW}<1.4$. Terminal seta less than twice the length of antennal segment 10 (figure 28G)

sauteri (Enderlein)

34 Rs vein with 12-22 setae

- Rs vein with 23-36 setae

35 Rs vein with inconspicuous setae (figure 22A-D)

- Rs vein with long setae (figure $21 \mathrm{~A}-\mathrm{C}$ ). 
36 Pterostigma slender and long; PT $>0.5 \mathrm{RL}$. Head with a distinct brown line connecting lateral and median ocelli.

- Pterostigma wide and short; PT $<0.5$ RL. Character combination different stigmaticalis $\mathrm{sp} . \mathrm{n}$. boehmeriae sp. $\mathrm{n}$.

$37 \mathrm{AL} / \mathrm{HW}<1.2, \mathrm{ML} / \mathrm{HW}<1.0$.

- $\mathrm{AL} / \mathrm{HW}>1.4, \mathrm{ML} / \mathrm{HW}>1.3$.

polaszeki sp. $\mathrm{n}$.

$38 \mathrm{AL} / \mathrm{HW}>1.62$. Antenna entirely brown. Apex of veins to forewing margin dark brown (figure 21C); pterostigma entirely brown. Female proctiger brown in the middle

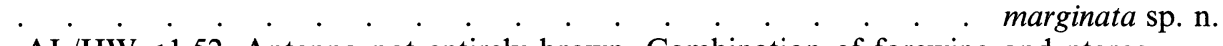

- AL/HW $<1.52$. Antenna not entirely brown. Combination of forewing and pterostigma coloration different. Female proctiger white in the middle

39 Surface spinules absent in cell c + sc. Male paramere rounded apically, widest at base, with a group of 7-9 stout setae in basal half of inner surface. muta sp. $\mathrm{n}$.

- Surface spinules present in cell c+sc. Male paramere pointed apically, widest in the middle, with a group of 1-2 stout setae at base of inner surface.

oceanica $\mathrm{sp} . \mathrm{n}$.

$40 \mathrm{AL} / \mathrm{HW}<1.35, \mathrm{ML} / \mathrm{HW}<1.4$. Rs vein with short setae (figure $21 \mathrm{D}$ ).

$-\dot{\mathrm{AL}} / \mathrm{HW}>1.50 . \dot{\mathrm{ML}} / \dot{\mathrm{HW}}>1.4$. Rs vein with long setae (figure $22 \mathrm{E}$ ).

palawanensis $\mathrm{sp} . \mathrm{n}$. setifera (Crawford)

41 Antennal rhinaria without bifid setae. $\mathrm{AL} / \mathrm{HW}>0.8$.

- Antennal rhinaria with bifid setae. AL/HW $<0.7$.

42 Antennal segment 7 with a long basal seta. Rs vein straight in apical half. . lii sp. $\mathrm{n}$.

- Antennal segment 7 without a long basal seta. Rs vein curved in apical half. . . . 43

43 Head with three pairs of tubercles on vertex. Forewing with brown patterns: an extensive darker one towards apex and two small lighter patterns, towards centre and in cell $\mathrm{cu}_{2}$ (figure 29C). stephaniella $\mathrm{sp} . \mathrm{n}$.

- Head without tubercles on vertex. Forewing with distinct brown colour at base and apex of pterostigma, at end of veins to forewing margin, at base of vein $\mathrm{A}$ and at middle parts of veins $A$ and $R$ (figure 29B).

. mathuri sp. n.

44 Antennal segment 8 with a long basal seta (figure 32H). . . brevicephala (Crawford)

- Antennal segment 8 without a long basal seta. . . . . . . . . . . . 45

45 Dorsum light yellow, venter of same coloration or darker. Male paramere long, robust and straight (figure $33 \mathrm{~K}, \mathrm{~L}$ ). $\mathrm{AL} / \mathrm{F} 1<4.78$. . . . . . . . . . . . . 46

- Dorsum dark brown to red, venter whitish. Male paramere slender and curved apically (figure $33 \mathrm{G}-\mathrm{J}$ ). $\mathrm{AL} / \mathrm{F} 1>4.78$.

46 Forewing transparent, setae on veins short (figure 32B). Surface spinules in cell c $+\mathrm{sc}$ confined to apex. Male paramere widest at base, with an apical hook on inner surface (figure 33L).

- Forewing light yellow, setae on veins long (figure 32A). Surface spinules in cell c + sc present at apex and extending towards distal half of vein $\mathrm{R}+\mathrm{M}+\mathrm{Cu}_{1}$ (figure $32 \mathrm{~A}$ ). Paramere widest at basal third, without an apical hook on inner surface (figure 33K). ambigua sp. $\mathrm{n}$.

47 Forewing transparent. Surface spinules absent in cell $\mathrm{c}+\mathrm{sc}$. Antennal rhinaria with long bifid setae (figure 32E) . martini sp. n.

- Forewing yellowish to brown. Surface spinules present in cell $\mathrm{c}+\mathrm{sc}$. Antennal rhinaria with short bifid setae (figure 32F).

48 Forewing cells densely covered in surface spinules; end of veins to forewing margin dark brown; veins R and A brown towards the middle (figure $31 \mathrm{C}$ ); WL/WW $<2.30$. 
- Forewing cells sparsely covered in surface spinules; end of veins to forewing margin with inconspicuous brown coloration; veins $\mathrm{R}$ and $\mathrm{A}$ not brown towards the middle (figure 31D); WL/WW $>2.35$.

sulawesiana sp. $\mathrm{n}$.

\section{Fifth instar larvae}

(Of the species where larvae are known, $P$. kleinhofiae Uichanco, $P$. russellae Mathur and $P$. sauteri (Enderlein) are excluded due to lack of material. The characters of $P$. mathuri sp. n. $=P$. cf. minuta are taken from Mathur, 1975.)

1 Body form elongate, $\mathrm{BL} / \mathrm{BW}>1.20$. $\mathrm{CW} / \mathrm{CL}>2$

- Body form rounded, $\mathrm{BL} / \mathrm{BW}<1.16 . \mathrm{CW} / \mathrm{CL}<2$

2 Antennal flagellum not segmented or subdivided. Sectasetae on outer margin of

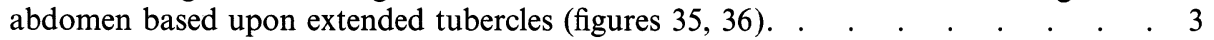

- Antennal flagellum indistinctly subdivided or segmented. Sectasetae on outer margin of abdomen not based on extended tubercles (figures 38, 43).

3 Apex of caudal plate tubercle with a distinct furrow in the middle (figure 36). On Malvaceae.

- Apex of caudal plate tubercle without a furrow in the middle (figure 35). On Clusiaceae

insolita sp. $\mathrm{n}$.

4 Dorsum of forewing pad with less than 12 sectasetae. On Gossypium sp.

. $\cdot$. . . . . . . . . . . . . . . . . . gossypii Russell

- Dorsum of forewing pad with more than 20 sectasetae . . . . . . . . . 5

5 Sectasetae on margin of caudal plate robust (figure 36B). On Urena lobata

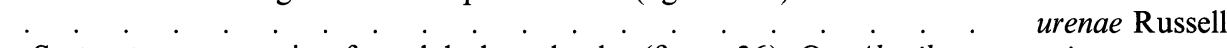

- Sectasetae on margin of caudal plate slender (figure 36). On Abutilon mauretianum.

abutili sp. $\mathrm{n}$.

6 Anal opening at the most posterior part of abdominal margin (figures 39, 40). . . 7

- Anal opening ventral (figure 41). . . . . . . . . . . . . . . . . . . 8

7 Forewing pads with few inconspicuous simple setae dorsally and marginally . . . .

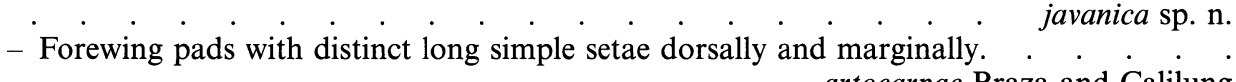

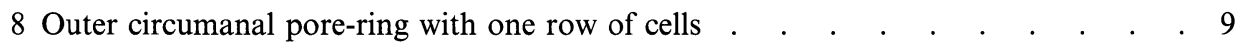

- Outer circumanal pore-ring laterally forming additional pores (figure 38 ) or pore fields connected to it (figure 42) .

9 Eyes not bulging out from margin . . . . . . . . . . psylloptera Crawford

- Eyes bulging out from margin . . . . . . . . . . . . . . . 10

10 On Trema orientalis (Ulmaceae). setifera (Crawford), P. dayak sp. n., P. palawanensis sp. n. and P. polaszeki sp. n.

- On Boehmeria nivea (Urticaceae) boehmeriae sp. $\mathrm{n}$.

11 Antenna 9-segmented. Outer circumanal pore-ring laterally forming two additional circular pore fields connected to it . . . . . . . . maculipennis Uichanco

- Antenna 3-segmented with subdivided flagellum. Outer circumanal pore-ring laterally with additional cells .

12 Dorsal surface with sectasetae and simple setae. Tarsal arolium expanded curvata sp. n.

- Dorsal surface with sectasetae only. Tarsal arolium elongate . . . . . . . 13

13 Third antennal segment $>4.6$ times segment 4 . . . . . . . bifasciata Kuwayama

- Third antennal segment $<4.5$ times segment 4 . . . . . . chonchaiensis Boselli

14 Anus terminal. Sectasetae present on dorsum and body margin . . . mathuri sp. n.

- Anus ventral. Sectasetae mainly confined to body margin . . . . . . . . 15 
15 Small stout setae on dorsal sclerites (except for caudal plate) numerous and very conspicuous . . . . . . . . . . . . . . . pterospermi $\mathrm{sp.} \mathrm{n}$.

- Small stout setae on dorsal sclerites (except for caudal plate) fewer in number and less conspicuous . . brevicephala (Crawford), robusta sp. n. and P. sulawesiana sp. $\mathrm{n}$.

\section{Species descriptions}

The species are treated below in alphabetical order. To keep the descriptions short, six types are described in detail, and reference to them is made in the individual species descriptions. These types do not reflect phylogenetic relationships. The distribution for each species is given on the basis of the examined material and of original literature records. Catalogues such as those of Hodkinson $(1983,1986)$ are not included.

The gossypii type includes all the nine Afrotropical species ( $P$. abutili sp. n., $P$. boxi sp. n., P. gossypii Russell, $P$. hollisi sp. n., P. insolita sp. n., P. lucida sp. n., $P$. medleri sp. n., $P$. sinuata sp. n. and $P$. urenae Russell) and one Oriental species (P. lienhardi sp. n.). Adult. Head short, AL/HW: 0.80-1.39; antenna 10-segmented, segments 4, 6, 8 and 9 each with a subapical rhinarium, rhinaria each with or without a special seta (except for $P$. lienhardi sp. n. with bifid setae on antennal rhinaria). WL/WW: 2.16-2.68. Metatibia without stout setae laterally (figure 4D); meracanthus short. First visible abdominal tergite with a patch of indistinct short spines laterally. Male proctiger with elongate or small rounded lateral plates posteriorly (figures 7A-C, 8A, B), inner surface with large peg-like setae (figure 3F, I) (lacking in $P$. insolita sp. n. and $P$. lienhardi sp. n. where instead long simple setae are present). Female genitalia straight apically, lateral valvulae membranous. Fifth instar larva. Body form elongate. Antenna 3-segmented. Humeral lobe absent. Dorsal sclerites covered by sectasetae as large as marginal ones, simple setae lacking. Caudal plate elongate, with large tubercle-like extensions marginally (figures 35, 36). Anus ventral, outer circumanal ring with a single row of pores (figure 35).

The kleinhofiae type includes four species (P. brendelli sp. n., P. elegans sp. n., $P$. kleinhofiae Uichanco and $P$. russellae Mathur). Adult. Head short, AL/HW: 0.55-0.83; antenna 8-segmented in P. elegans sp. n., segments 3, 4, 6 and 7 each with a subapical rhinarium; antenna 9-segmented in $P$. kleinhofiae Uichanco, segments 3, 5, 7 and 8 each with a subapical rhinarium; antenna 10-segmented in $P$. brendelli sp. n. and $P$. russellae Mathur, segments 4, 6, 8 and 9 each with a subapical rhinarium. A bifid seta present (except in $P$. brendelli sp. n.) on some or all rhinaria. WL/WW: 2.12-2.48. Metatibia with one to three stout setae laterally similar to apical metatibial spurs (absent in $P$. brendelli sp. n.); meracanthus short. First visible abdominal tergite with a patch of distinct long spines laterally. Male proctiger with elongate to rounded lateral plates posteriorly (figure 10A, B), inner surface without stout setae. Female genitalia straight apically, lateral valvulae membranous. Adults are characterized by a distinct horn-like structure on each tergite. Fifth instar larva. No material was available for study.

The psylloptera type includes 18 species $(P$. boehmeriae sp. n., $P$. dayak sp. n., $P$. distincta sp. n., P. longiantennata sp. n., P. macrochaetis sp. n., P. maculipennis, $P$. marginata sp. n., $P$. muiri sp. n., $P$. muta sp. n., $P$. oceanica sp. n., $P$. palawanensis sp. n., $P$. papuana sp. n., $P$. polaszeki sp. n., $P$. psylloptera Crawford, $P$. sauteri (Enderlein), P. setifera (Crawford), P. stigmaticalis sp. n. and $P$. trematos Yang et al.). Adult. Head short, AL/HW: 1.08-2.38; antenna 10-segmented, segments 4, 
6,8 and 9 each with a subapical rhinarium, rhinaria each with or without a special seta. WL/WW: 2.12-2.43. Metatibia with stout setae laterally, similar to apical metatibial spurs (figure 4C, F); meracanthus long, curved or straight laterally. First visible abdominal tergite on either side with a stalked structure bearing long spines (figure 4A, B). Male proctiger with elongate lateral plates posteriorly (figure 23). Male paramere with inner face bearing stout setae in basal two-thirds, two hooks apically (figure $3 \mathrm{E}$ ). Female genitalia straight apically, lateral valvulae membranous. Fifth instar larva. Body form elongate. Antenna 3-segmented (5-segmented in $P$. boehmeriae sp. n. and 9-segmented in P. maculipennis Uichanco), flagellum often indistinctly subdivided. Humeral lobe absent. Dorsal sclerites covered by sectasetae as large as marginal ones and few inconspicuous simple setae. Caudal plate elongate, with shallow or no tubercle-like extensions marginally. Anus ventral, outer circumanal ring with a single row of pores (in P. maculipennis Uichanco strongly expanded laterally, figure 42).

The chonchaiensis type includes three species ( $P$. bifasciata Kuwayama, P. chonchaiensis Boselli and $P$. phalaki Mathur). Morphologically similar to the psylloptera type from which it differs as follows. Adult. Forewing bearing two brown bands (figure 12A, B), one in the middle (from costal break to anal break) and one apically (from apical third of $\mathrm{Rs}$ vein towards apical half of vein $\mathrm{Cu}_{1 \mathrm{a}}$ ). First visible abdominal tergite with a patch of indistinct short spines laterally. Fifth instar larva. Outer circumanal ring with a single row of pores, with multipores laterally (figure 37).

The wilderi type includes six species ( $P$. artocarpae Braza and Calilung, $P$. calcarata sp. n., P. curvata sp. n., P. javanica sp. n., P. similis $\mathrm{sp}$. n. and $P$. wilderi Crawford). Adult. Head short, AL/HW: 1.09-1.55; antenna 10-segmented, segments 4, 6, 8 and 9 each with a subapical rhinarium, rhinaria each with or without a special seta. WL/WW: 2.10-2.42. Metatibia with stout setae laterally similar to apical metatibial spurs (reduced in number in $P$. wilderi Crawford and $P$. calcarata sp. n.); meracanthus long, often straight laterally. First visible abdominal tergite with a patch of indistinct short spines laterally. Male proctiger without lateral plates posteriorly (figure 14A-F), inner surface with stout setae in basal two-thirds (lacking in $P$. curvata sp. n.), often with two hooks apically. Female genitalia straight apically, lateral valvulae membranous. Fifth instar larva. Body form elongate. Antenna 3-segmented, flagellum often indistinctly subdivided. Humeral lobe absent. Dorsal sclerites covered by sectasetae as large as marginal ones and short to long simple setae. Caudal plate elongate, without tubercle-like extensions marginally. Anus terminal, outer circumanal ring with a single row of pores having multipores at irregular intervals (figures 39, 40) or anus ventral, outer circumanal ring laterally expanded with multipores (figure 38 ).

The brevicephala type includes $P$. ambigua sp. n., $P$. brevicephala (Crawford), $P$. lii sp. n., P. martini sp. n., P. mathuri sp. n., P. minuta Crawford, P. pterospermi sp. n., $P$. robusta sp. n., $P$. stephaniella sp. n. and $P$. sulawesiana sp. Adult. Head distinctly short, antenna 8-segmented, segments $3,4,6$ and 7 each with a subapical rhinarium, rhinaria each with or without a special seta. WL/WW: $2.12-2.52$. Metatibia with few stout setae laterally, similar to apical metatibial spurs (figure 4E, $\mathrm{H}$ ); meracanthus small. First visible abdominal tergite with a patch of often distinct spines laterally. Male proctiger without lateral plates posteriorly (figure $33 \mathrm{~A}-\mathrm{F}$ ), inner surface of paramere without stout setae (figure 33J, K). Female genitalia upturned apically, lateral valvulae sclerotized, serrated ventrally. 
Paurocephala abutili sp. n.

(figures $5 \mathrm{~A}, \mathrm{E}, 8 \mathrm{G}, 36$ )

Description. Species of the gossypii type.

Adult: head brown, yellow towards genae; vertex covered by inconspicuous setae. Clypeus yellow to brown. Antenna yellow, segments 9 and 10 brown; segment 9 without a long basal seta. Thorax brown and covered by inconspicuous setae dorsally, yellow laterally and ventrally. Metascutellar horn small, blunt apically. Forewing transparent to light yellow; veins yellow, apically brown to forewing margin, base and central part of vein A and central part of vein R brown; base of pterostigma brown. Forewing with inconspicuous setae on veins (vein Rs with 19-23 setae); Rs vein marginally straight, curved in apical third. Surface spinules present in all cells of forewing; radular spinules lacking. Legs yellow, tarsal segments light brown. Meracanthus short, laterally straight, rounded apically. Abdominal tergites brown, sternites and genitalia yellow. Male paramere thin, straight in basal half, tapering in distal half and bend posteriorly, few short setae posteriorly visible from lateral view; inner surface with three to five peg-like setae situated between basal fifth and distal third. Measurements and ratios as in table $2 \mathrm{~A}-\mathrm{C}$.

Fifth instar larva: colour unknown. Antenna 3-segmented; segments 1 and 2 each with one sectaseta, segment 3 with 7 sectasetae grouped in 3, 3 and 1. Dorsal sclerites covered by sectasetae as large as marginal ones, simple setae lacking. Forewing pad with 18-22 sectasetae marginally; humeral lobe lacking. Caudal plate with large tubercle-like extensions marginally, apical one ending in a long process longitudinally divided; sectasetae present dorsally and marginally. Arolium triangular and petiolate, expanded basally. Anus ventral, outer circumanal ring with a single row of pores. Measurements and ratios as in table 3.

Host plants. Abutilon mauritianum (Jacq.) Medik. (Malvaceae).

Table 3. Measurements and ratios of last instar larvae Paurocephala species $(N=$ number of measured specimens).

\begin{tabular}{lccccccc}
\hline Species & $N$ & $\mathrm{BL}$ & $\mathrm{AL}$ & $\mathrm{CW}$ & $\mathrm{BL} / \mathrm{BW}$ & $\mathrm{AL} / \mathrm{FL}$ & $\mathrm{CW} / \mathrm{CL}$ \\
\hline abutili & 2 & $1.37-1.49$ & $0.41-0.42$ & 0.52 & $1.33-1.36$ & $0.96-1.02$ & $1.25-1.28$ \\
artocarpae & 5 & $1.37-1.80$ & $0.62-0.67$ & $0.58-0.60$ & $1.14-1.40$ & $1.12-1.32$ & $1.39-1.51$ \\
boehmeriae & 3 & $1.41-1.51$ & $0.71-0.73$ & $0.39-0.41$ & $1.53-1.55$ & $1.43-1.63$ & $0.91-1.00$ \\
brevicephala & 5 & $1.37-1.63$ & $0.42-0.44$ & $0.70-0.83$ & $1.05-1.16$ & $0.71-0.80$ & $2.17-2.26$ \\
bifasciata & 3 & $1.59-1.73$ & $0.75-0.76$ & 0.50 & $1.42-1.50$ & $1.27-1.45$ & 1.20 \\
conchaiensis & 3 & $1.24-1.49$ & $0.63-0.64$ & $0.44-0.55$ & $1.31-1.33$ & $1.26-1.46$ & $1.24-1.45$ \\
curvata & 5 & $1.34-1.46$ & $0.58-0.61$ & $0.52-0.58$ & $1.30-1.49$ & $1.17-1.26$ & $1.57-1.79$ \\
dayak & 2 & $1.46-1.59$ & $0.60-0.61$ & $0.50-0.53$ & $1.36-1.41$ & $1.19-1.30$ & $1.18-1.30$ \\
gossypii & 2 & $1.17-1.22$ & $0.35-0.37$ & $0.50-0.51$ & $1.26-1.32$ & $0.95-0.98$ & $1.42-1.43$ \\
insolita & 3 & $1.37-1.66$ & $0.36-0.38$ & $0.50-0.53$ & $1.35-1.45$ & $0.83-0.93$ & $1.15-1.24$ \\
javanica & 2 & $1.71-1.85$ & $0.61-0.64$ & 0.58 & $1.36-1.40$ & $1.24-1.25$ & $1.53-1.59$ \\
maculipennis & 3 & $1.73-1.90$ & $1.07-1.13$ & $0.61-0.68$ & $1.40-1.54$ & $1.76-1.95$ & $1.27-1.63$ \\
palawanensis & 2 & $1.70-1.81$ & $0.63-0.65$ & $0.57-0.61$ & $1.28-1.52$ & $1.17-1.26$ & $1.41-1.43$ \\
polaszeki & 2 & $1.10-1.17$ & $0.54-0.55$ & $0.40-0.41$ & $1.18-1.20$ & $1.18-1.24$ & $0.98-1.08$ \\
psylloptera & 2 & $1.59-1.63$ & $0.70-0.73$ & 0.49 & $1.49-1.55$ & $1.45-1.54$ & 1.08 \\
pterospermi & 5 & $1.45-1.57$ & $0.42-0.45$ & $0.74-0.82$ & $1.02-1.11$ & $0.80-0.86$ & $2.17-2.35$ \\
robusta & 4 & $1.32-1.59$ & $0.41-0.45$ & $0.64-0.77$ & $1.10-1.13$ & $0.81-0.91$ & $2.03-2.28$ \\
setifera & 2 & $1.40-1.65$ & $0.71-0.76$ & $0.41-0.55$ & $1.50-1.61$ & $1.51-1.69$ & $1.02-1.09$ \\
sulawesiana & 5 & $1.27-1.44$ & $0.42-0.45$ & $0.67-0.82$ & $0.98-1.13$ & $0.78-0.83$ & $2.09-2.31$ \\
urenae & 2 & $1.20-1.27$ & $0.39-0.42$ & $0.51-0.53$ & $1.29-1.39$ & $0.85-1.05$ & $1.29-1.33$ \\
\hline
\end{tabular}


Distribution. Kenya.

Material examined. HolotyPE đ̊, Kenya: Nairobi (near National Museum), 5500 ft, 6 January 1972 (C. F. Huggins) (BMNH). Dry mounted.

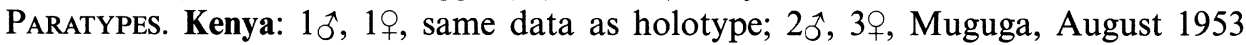
(V. F. Eastop); 2ᄎ, 1, 4 larvae, Chiromo, 22 November 1969, Abutilon mauritianum (H. Schmutterer); 1ð, 2ᄋ, 2 larval exuvia, Sultan Hamud, 15 May 1988 (J. H. Martin) (BMNH, NHMB). Dry and slide mounted.

Comments. Similar to $P$. urenae Russell from which it is distinguished in the adult by the darker body coloration and different antennal shape, and the more slender sectasetae in the larva.

Table 4. Adult and larval characters of Paurocephala spp. (cf. tables 5, 6, figures 47, 48).

Adult

1. Head moderately short (0); very short (1).

2. Antenna 10-segmented (0); 8- or 9-segmented (1).

3. Antenna longer than head width (0); as long as or shorter than head width (1).

4. Antennal rhinaria without or with one special seta (0); with a bifid seta (1).

5. Antennal segment 9 (when 10 -segmented) without a long basal seta (0); with a long basal seta (1).

6. Longer terminal seta shorter than combined length of last three antennal segments $(0)$; longer (1).

7. Apical segment of labium less than half length of basal segment (0); more (1).

8. Forewing without colour pattern, with one or three bands $(0)$; with each a distinct brown band in the middle and apically (1).

9. Meracanthus short (0); long (1).

10. Metatibia distinctly longer than metafemur (0); about as long as or shorter (1).

11. Metatibia with simple setae laterally (0); with strongly thickened setae (1).

12. Abdominal tergites lacking a horn-like dorsal structure $(0)$; each tergite with a horn-like dorsal structure (1).

13. First visible abdominal tergite with lateral patch of short to long spines (0); spines on stalk-like structure (1).

14. Male proctiger without (0); with distinct posterior scerites (1).

15. Inner surface of paramere with simple setae (0); with stout setae (1); with peg-like setae (2).

16. Head of aedeagus well-differentiated from stem (0); not distinctly differentiated from stem (1).

17. Female proctiger with well-defined, sickle-shaped apical process (0); evenly tapering apically (1).

18. Circumanal ring with one row of pores $(0)$; with two or more rows of pores (1).

19. Lateral valvulae membranous (0); sclerotized with ventral serration (1).

Larva

20. Body form elongate (0); circular (1).

21. Sectasetae confined to body margin, if present on dorsum then small in size (0); large, present marginally and dorsally (1).

22. Simple setae on dorsum indistinct (0); distinct (1).

23. Antennal segment 2 without sectasetae (0); with (1).

24. Thoracic dorsal sclerites not fused to forewing pads (0); fused (1).

25. Humeral lobe of forewing pad absent (0); present (1).

26. Marginal sectasetae on caudal plate not or on shallow tubercles $(0)$; on large tubercles (1).

27. Anus terminal (0); ventral (1).

28. Outer circumanal ring with a single row of pores $(0)$; with one row laterally expanded with multipores (1). 
Table 5. Matrix for Paurocephala spp. (adult characters) (cf. table 4, figure 47).

\begin{tabular}{llll}
\hline $\begin{array}{l}\text { Diclidophlebia dahli } \\
\text { abutili }\end{array}$ & 0000000000000000000 & marginata & 0000001010101110110 \\
ambigua & $000000100000012 ? 110$ & martini & $1111 ? 10001100001001$ \\
artocarpae & $1111 ? 10001100001001$ & mathuri & $1110 ? 00000100001001$ \\
bifasciata & 0000001010100010110 & medleri & $00 ? 0001000000120110$ \\
boehmeriae & 0000101110100110110 & minuta & $111 ? ? ? 000 ? 1000 ? ? 0 ? 1$ \\
boxi & $00000010101011101 ? 0$ & muiri & 0000101010101110110 \\
brendelli & $00 ? 0001000000120110$ & muta & 0000001010101110110 \\
brevicephala & $00100000010101001 ? 0$ & oceanica & 0000001010101110110 \\
calcarata & $1111 ? 10001100001001$ & palawanensis & 0000001010101110110 \\
chonchaiensis & 0000001010100010100 & papuana & 0000101010101110110 \\
curvata & 0000101110100110110 & phalaki & $00001011101001 ? 01 ? 0$ \\
dayak & 0000000010100000100 & polaszeki & 0000001010101110110 \\
distincta & 0000101010101110110 & psylloptera & 0000101010101110110 \\
elegans & 0000101010101100110 & pterospermi & $1111 ? 10001100001001$ \\
gossypii & $0111 ? 10000110100100$ & robusta & $1111 ? 10001100001001$ \\
hollisi & 0010001000000120110 & russellae & $001 ? ? 0 ? 0001101 ? 01 ? 0$ \\
insolita & 0000001000000120110 & sauteri & 0000101010101110110 \\
javanica & 0010001000000101110 & setifera & 0000001010101110110 \\
kleinhofiae & 0000001010100010110 & similis & 0000001010100010110 \\
lienhardi & $0111 ? 00000110100100$ & sinuata & 0000001000000120110 \\
lii & 0001000000000100110 & stephaniella & $1110 ? 00001100001 ? ? 1$ \\
longiantennata & $1110 ? 00000100001001$ & stigmaticalis & $00000010101011 ? 0110$ \\
lucida & 0000101010101110110 & sulawesiana & $1111 ? 10001100001001$ \\
macrochaetis & $0000001000000 ? ? ? 110$ & trematos & 0000101010101110110 \\
maculipennis & 0000101010101110110 & urenae & 0000001000000120110 \\
\hline & 0000001010101110110 & wilderi & 0000000010100010100 \\
\hline
\end{tabular}

Table 6. Matrix for selected Paurocephala spp. (adult and larval characters) (cf. table 4, figure 48).

Diclidophlebia dahli
abutili
artocarpae
bifasciata
boehmeriae
brevicephala
chonchaiensis
curvata
dayak
gossypii
insolita
javanica
kleinhofiae
maculipennis
mathuri
palawanensis
phalaki
polaszeki
psylloptera
pterospermi
robusta
russellae
setifera
sulawesiana
urenae

$0000000000000000000 ? 100011 ? 000$

$000000100000012 ? 11001100100100$

000000101010001011010110111000

000010111010011011011100102000

$00000010101011101 ? 011100100000$

$1111 ? 1000110000100101001010011$

000010111010011011011100102000

000000001010000010011110102010

000010101010111011011100100000

001000100000012011001100100100

001000100000010111001100100100

000000101010001011010110111000

0111?0000011010010011100100000

000000101010111011010100103000

1110?00000100001001?1101?00001

000000101010111011011100100000

$00001011101001 ? 01 ? 011100102000$

000000101010111011011100100000

000010101010111011011100100000

1111?1000110000100101001010011

$1111 ? 1000110000100101001010011$

$001 ? ? 0 ? 0001101 ? 01 ? 0111 ? 0100 ? 00$

000000101010111011011100100000

1111 ? 1000110000100101001010011

000000100000012011001100100100 
Paurocephala ambigua sp. $\mathrm{n}$.

(figures 32A, C, I, 33E, K, 34E)

Description. Species of the brevicephala type.

Adult: head orange, sides along coronal suture white; vertex covered by short setae. Clypeus brown. Antenna yellow to orange, segments 7 and 8 brown; segment 7 with a long basal seta. Thorax orange to brown and covered by short setae dorsally, yellow to light brown laterally and ventrally. Metascutellar horn small, wide basally, blunt apically. Forewing transparent to light yellow; veins yellow, apically brown to forewing margin, central part of veins A and R brown; pterostigma white, brown apically. Forewing with long setae on veins (vein Rs with 16-19 setae); Rs vein marginally curved in apical third. Surface spinules present in all cells of forewing, reduced in cell $\mathrm{c}+\mathrm{sc}$ (confined at apex and extending towards distal half of vein $\mathrm{R}+\mathrm{M}+\mathrm{Cu}_{1}$ ); radular spinules lacking. Legs yellow to light brown, tarsal segments brown. Meracanthus short, straight laterally, pointed apically. Abdomen, including genitalia, orange to brown. Male paramere elongate, widest basally and tapering apically, bend posteriorly in apical half, few long setae posteriorly visible from lateral view. Measurements and ratios as in table $2 \mathrm{~A}-\mathrm{C}$.

Fifth instar larva: unknown.

Host plants. Unknown.

Distribution. Indonesia (Sulawesi).

Material examined. Holotype ô, Indonesia: Sulawesi, Sulawesi Utara, DumogaBone N. P., 26 February 1985 (R. Ent. Soc. Lond., Project Wallace) (BMNH). Dry mounted.

PARATYPES. Indonesia: $1 \hat{\jmath}$, same data as holotype; $3 \hat{\jmath}$, same data but 5-12 and 21 February $1985 ; 1$, same data but $400 \mathrm{~m}, 11$ February $1985 ; 1$, same data but base camp area, ca 190 m, 3-4 February 1985; 19, same data but 15 June 1985; 1 , same data but 9-16 May 1985 (forest edge-yellow pan traps); 19, same data but April 1985 (grassland and river bank) (J. S. Noyes); 19, same data but 1 April 1985 (light trap) (H. Barlow); 4ðิ, 5 ㅇ same data but Gunung Mogogonipa, ca 400-500 m, 8 April 1985 (J. H. Martin) (BMNH, NHMB). Dry and slide mounted.

Comments. Similar to $P$. pterospermi sp. n. from which it differs in the shape of the paramere and setiferation on forewing and body.

\section{Paurocephala artocarpae Braza and Calilung}

(figures 13B, 14B, 15B, H, 16B, 39)

Paurocephala artocarpae Braza and Calilung, 1981: 336. Holotype $\hat{\sigma}$, Philippines: Luzon, Los Baños (near Laguna), 10 January 1976, Artocarpus blancoi (A. B. Bragado) (UPPC), not examined.

Description. Species of the wilderi type.

Adult: head yellow to brown; vertex covered by long setae. Clypeus yellow. Antenna yellow, segments 4 and 6, apical half of segment 8 and entire segments 9 and 10 brown; segment 9 without a long basal seta. Thorax yellow to light brown and covered by long setae dorsally, light brown to white laterally and ventrally. Metascutellar horn small, blunt apically. Forewing light brown; veins brown; pterostigma white to light brown. Forewing with long setae on veins (vein Rs with 18-23 setae); Rs vein marginally curved throughout entire length. Surface spinules present in all cells of forewing; radular spinules present in $\mathrm{m}_{2}$, reduced in $\mathrm{cu}_{1}$. Legs white to yellow, tarsal segments light brown. Meracanthus long, straight laterally, rounded 
apically. Abdomen including genitalia, light brown. Male paramere long and straight posteriorly, widest in the middle and tapering apically, few short setae posteriorly visible from lateral view; inner surface with two hooks apically and rows of stout setae confined posteriorly in basal half. Measurements and ratios as in table $2 \mathrm{~A}-\mathrm{C}$.

Fifth instar larva: antenna yellow to light brown; 3-segmented, flagellum indistinctly subdivided; segment 1 and 2 without sectasetae, flagellum with five sectasetae grouped in 2, 2 and 1. Thoracic tergites yellow to light brown; abdominal tergites brown. Dorsal sclerites covered by sectasetae and simple setae as large as marginal ones. Wing pads yellow to white, brown towards thorax; forewing pad with three to six sectasetae and four to eight simple setae marginally; humeral lobe lacking. Caudal plate yellow, rounded marginally; sectasetae present dorsally and marginally. Arolium triangular and petiolate, expanded basally. Anus terminal, outer circumanal ring with a single row of pores, subdivided at irregular intervals. Measurements and ratios as in table 3 .

Host plants. Artocarpus blancoi (Elm.) Merr. (Moraceae) (Braza and Calilung, 1981).

Distribution. Philippines (Luzon) (Braza and Calilung, 1981), Malaysia (Sabah) and Indonesia (Sulawesi).

Material examined. Philippines: 20ิ, 2ㅇ, Luzon, Los Baños (near Laguna), 10 January 1976, Artocarpus blancoi (R. D. Braza). Indonesia: 14 $\hat{\circ}, 13 q$ and numerous larvae, Sulawesi, Sulawesi Utara, Dumoga-Bone N. P., Mogogonipa Gunung, ca 400-500 m, 8 April 1985, Artocarpus sp., ant attended (J. H. Martin); 2ᄋ, same data but 6-13 July 1985 and 2-9 October 1985 (yellow pan traps); 2へ, same data but ca $900 \mathrm{~m}$, Clark's Camp ridge, 13 April 1985. Malaysia: 2 , Sabah, Poring Hot Springs, 500 m, 6 May 1987 (D. Burckhardt and I. Löbl) (BMNH, NHMB). Dry and slide mounted and preserved in alcohol.

Comments. Type material was unavailable for study but material collected with the type series was examined. The material from the Philippines has a straight vein $\mathrm{M}_{1+2}$ which is directed away from apical part of vein $\mathrm{Rs}$ while the material from Sabah and Sulawesi has a marginally curved vein $\mathrm{M}_{1+2}$ which is parallel to the apical part of Rs vein. This difference is here considered as intraspecific variation.

\section{Paurocephala bifasciata Kuwayama, stat. rev.}

$$
\text { (figures 12A, C, E, G, I, 37) }
$$

Paurocephala bifasciata Kuwayama, 1931: 119. Syntypes, Taiwan: Taihoku, 28 May 1920 and 16 October 1923, Ficus erecta var. beecheyana (R. Takahashi) (EIHU), not examined. Synonymized with $P$. chonchaiensis Boselli, by Sasaki, 1954: 31.

Description. Species of the chonchaiensis type.

Adult: head yellow, coronal suture and areas around median and lateral ocelli brown; vertex covered by long setae. Clypeus brown. Antenna yellow, distal half of segment 4 , distal two-thirds of segment 6 , distal half of segment 8 and entire segments 9 and 10 brown; segment 9 with a long seta in basal quarter. Thorax brown and covered by long setae dorsally, yellow to brown laterally and ventrally. Metascutellar horn large, pointed apically. Forewing including pterostigma transparent with two brown bands, from costal break to anal break and towards apex, from apical third of $\mathrm{Rs}$ vein towards apical half of $\mathrm{Cu}_{1 \mathrm{a}}$ vein, often a small light brown spot present in cell $\mathrm{cu}_{2}$; veins light brown, apically brown to forewing margin. Forewing with short setae on veins (vein Rs with 17-20 setae); Rs vein strongly curved at apical 
third. Surface spinules present in all cells of forewing; radular spinules present in $\mathrm{m}_{2}$, reduced in $\mathrm{cu}_{1}$. Legs yellow, last tarsal segment dark brown. Meracanthus long, almost straight laterally, rounded apically. Abdominal tergites brown, sternites and genitalia, yellow to white. Male paramere short and straight, truncate to rounded apically, few short setae posteriorly visible from lateral view; inner surface with two hooks apically, one to three stout setae basally. Measurements and ratios as in table $2 \mathrm{~A}-\mathrm{C}$.

Fifth instar larva: colour white to light yellow throughout. Antenna 3-segmented, flagellum indistinctly subdivided; segments 1 without a sectaseta, segment 2 with one sectaseta, flagellum with five sectasetae grouped in 2, 2 and 1. Dorsal sclerites covered by sectasetae as large as marginal ones and few inconspicuous simple setae. Forewing pad with seven to nine sectasetae and one or two inconspicuous simple setae marginally; humeral lobe lacking. Caudal plate rounded marginally, apically with a shallow excavated grove; sectasetae present dorsally and marginally. Arolium triangular and petiolate, extended basally. Anus ventral, outer circumanal ring with a single row of pores, laterally multiporous. Measurements and ratios as in table 3 .

Host plants. Paurocephala bifasciata was described from material collected on Ficus erecta var. beecheyana (Hook. and Arn.) King. Uye (1937) recorded the species from Ficus foveolata Pittier and material was recently collected on Covellia hispida (L. f.) Miq. (Moraceae).

Distribution. China (Hong Kong), Taiwan (Kuwayama, 1931; Yang, 1984 pro parte (p.p.), Japan (Kyushu) (Uye, 1937) (Shikoku and Honshu) (Miyatake, 1964) (Ishigaki and Iriomote Islands) (Miyatake, 1965) and Vietnam (Klimaszewski, 1970). Other records in the literature are erroneous (see under comments).

Material examined. China: $4 \hat{\jmath}$, 4९, 2 larvae, Hong Kong, New Territories, 13 May 1974, Covellia hispida (D. S. Hill); 10, same data but Shing Mun Country Park, 250-320 m, 4 December 1988 (C. Lienhard); 80, 11우, 13 larvae, Hong Kong Island,

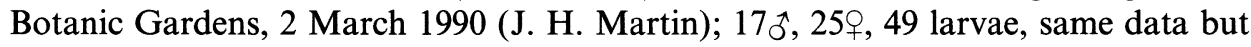
the Peak tram station, 25 November 1999. Taiwan: $2 \hat{0}$, 2o , Nantou, Kuantaochi, 26 June 1979, Ficus erecta var. beecheyana (C. T. Yang). Japan: 10, 4우, Kyushu, Mt. Wakasugi, near Fukuoka, 16 February and 5 April 1958, Ficus foveolata (Y. Miyatake). Vietnam: 1ㅇ, Lan Dong Province, $14 \mathrm{~km} \mathrm{~S} \mathrm{of} \mathrm{Bao} \mathrm{Loc,} 7$ May 1997 (K. Matsumoto) (BMNH, MHNG, NHMB, NMNS, OMNH, TULE). Dry and slide mounted and preserved in alcohol.

Comments. A number of records citing $P$. chonchaiensis Boselli may in fact concern partly or entirely $P$. bifasciata (e.g. Miyatake, 1964, 1965; Klimaszewski, 1970; Yang, 1984 p.p.; Takahashi, 1936 p.p.).

Paurocephala boehmeriae sp. $\mathrm{n}$.

(figures 22C, 23H, 24K, 25H, 27A, 28H, M, 43)

Description. Species of the psylloptera type.

Adult: head brown, vertex with a pair of yellow spots along base of coronal suture; vertex covered by short setae. Clypeus brown. Antenna yellow, apex of segments 4 and 6 and entire segments $8-10$ brown; segment 9 without a long basal seta. Thorax brown with yellow markings along body axis and covered by short setae dorsally, brown to light brown laterally and ventrally. Metascutellar horn small, pointed apically. Forewing transparent; veins light brown; pterostigma white, often with a central transparent area. Forewing with inconspicuous setae on veins 
(vein Rs with 13-21 setae); Rs vein marginally curved in apical half. Surface spinules confined to cells in apical half of forewing; radular spinules present in $\mathrm{m}_{2}$, reduced in $\mathrm{cu}_{1}$. Legs white to yellow, tarsal segments dark brown. Meracanthus long, curved laterally, pointed apically. Abdominal tergites dark brown, sternites white, genitalia yellow to brown. Male paramere long and straight posteriorly, widest in the middle, rounded apically, numerous long setae posteriorly visible from lateral view; inner surface with two hooks apically, two to five stout setae in basal quarter. Measurements and ratios as in table $2 \mathrm{~A}-\mathrm{C}$.

Fifth instar larva: antenna yellow, distal third of flagellum brown; indistinctly 5-segmented; segment 1 without a sectaseta, segment 2 with one sectaseta, flagellum with five sectasetae grouped in 2, 2 and 1. Dorsal sclerites light brown, covered by sectasetae as large as marginal ones and few inconspicuous simple setae. Wing pads yellow to light brown; forewing pad with eight or nine sectasetae and one or two inconspicuous simple setae marginally; humeral lobe lacking. Caudal plate brown, elongate with small tubercle-like extensions marginally, apical one ending in a relatively long process excavated $\mathrm{V}$-shaped apically; sectasetae present dorsally and marginally. Arolium triangular and petiolate, distinctly expanded basally. Anus ventral, outer circumanal ring with a single row of pores. Measurements and ratios as in table 3 .

Host plants. Boehmeria nivea (L.) Gaudich (Urticaceae).

Distribution. China (Hong Kong).

Material examined. Holotype $\hat{\jmath}$, China: Hong Kong, Lantau, Tung Chung, 26 November 1999, Boehmeria nivea (J. H. Martin) (BMNH). Dry mounted.

PARATYPES. China: $25 \hat{\jmath}, 30$ 우 and numerous larvae, same data as holotype (BMNH, NHMB). Dry and slide mounted and preserved in alcohol.

Comments. Adult P. boehmeriae sp. n. are similar to P. sauteri (Enderlein) but differ in the absence of a long basal seta on antennal segment 9 . The larva resembles that of $P$. setifera (Crawford) from which it can be best separated by the different host plant.

\section{Paurocephala boxi sp. $\mathrm{n}$.}

(figures $6 \mathrm{C}, \mathrm{H}, 8 \mathrm{~A}, \mathrm{C}, \mathrm{E}, \mathrm{M}$ )

Description. Species of the gossypii type.

Adult: head yellow; vertex covered by inconspicuous setae. Clypeus yellow to brown. Antenna yellow, segments 9 and 10 brown; segment 9 without a long basal seta. Thorax entirely yellow and covered by inconspicuous setae dorsally. Metascutellar horn small, blunt apically. Forewing including pterostigma transparent; veins yellow, apically light brown to forewing margin, central part of vein A brown. Forewing with inconspicuous setae on veins (vein Rs with 14-16 setae); Rs vein marginally curved in apical third. Surface spinules present in all cells of forewing; radular spinules lacking. Legs yellow, tarsal segments light brown. Meracanthus small, curved laterally, rounded apically. Abdomen including genitalia, yellow. Male paramere straight posteriorly, widest in basal third, tapering towards apex and rounded apically, few short setae posteriorly visible from lateral view; inner surface with one or two peg-like setae at basal third. Measurements and ratios as in table $2 \mathrm{~A}-\mathrm{C}$.

Fifth instar larva: unknown. 
Host plants. Adults were collected on Sterculia tragacantha Lindl. (Sterculiaceae) which could be the host plant.

Distribution. Ghana.

Material examined. Holotype $\widehat{\jmath}$, Ghana: Tafo, December 1942, Sterculia tragacantha (H. E. Box) (BMNH). Dry mounted.

PARATYPes. Ghana: $2 \widehat{\jmath}, 2 q$, same data as holotype (BMNH, NHMB). Dry and slide mounted.

Comments. Similar to P. medleri sp. $\mathrm{n}$. from which it is easily distinguished by the shape of the male paramere.

\section{Paurocephala brendelli sp. $\mathrm{n}$.}

(figures 9E, 11B, D, E, G, I)

Description. Species of the kleinhofiae type.

Adult: head orange, sides along coronal suture and area around median ocellus white; vertex covered by short setae. Clypeus orange. Antenna yellow to orange, segments 9 and 10 brown; segment 9 without a long basal seta. Thorax orange and covered by inconspicuous setae dorsally, yellow to orange laterally and ventrally. Metascutellar horn large, pointed apically. Forewing yellow to orange with small rounded transparent areas; veins yellow, apically brown to forewing margin; pterostigma white, brown basally and apically. Forewing with short setae on veins (vein Rs with 28-35 setae); Rs vein marginally curved basally and in distal quarter. Surface spinules present in all cells of forewing; radular spinules lacking. Legs including tarsal segments, yellow to orange. Meracanthus short, straight laterally, rounded apically. Abdomen including genitalia, orange to brown. Male paramere rounded, widest at apical two-thirds, few short setae posteriorly visible from lateral view. Measurements and ratios as in table $2 \mathrm{~A}-\mathrm{C}$.

Fifth instar larva: unknown.

Host plants. Unknown.

Distribution. Malaysia (Malaysian Peninsula).

Material examined. Holotype $\hat{0}$, Malaysia: Malaya, Negeri Sembilan, Pasoh Forest Reserve, 21 September 1994 (M. J. D. Brendell) (BMNH). Dry mounted.

Paratypes. Malaysia: $10 \hat{\jmath}, 3 q$, same data as holotype (BMNH, NHMB). Dry and slide mounted.

Comments. P. brendelli sp. $\mathrm{n}$. is similar to P. kleinhofiae Uichanco from which it differs in the 10-segmented antennae.

\section{Paurocephala brevicephala (Crawford)}

(figures 31A, 32H, N, 33A, G, 34A)

Pauropsylla brevicephala Crawford, 1917: 163. Lectotype 9 , examined, by present designation. Paurocephala brevicephala (Crawford); Crawford, 1919: 150.

Description. Species of the brevicephala type.

Adult: head brown, yellow towards genae; vertex covered by short setae. Clypeus brown. Antenna yellow; segments 7 and 8 with a long basal seta. Thorax brown and covered by inconspicuous setae dorsally, yellow to brown laterally and ventrally. Metascutellar horn short, wide basally, blunt apically. Forewing light brown; veins yellow, apically light brown to forewing margin, vein $\mathrm{C}+\mathrm{Sc}$ brown; pterostigma white. Forewing with short setae on veins (vein Rs with 14-19 setae); Rs vein marginally curved throughout. Surface spinules present in all cells of forewing, 
reduced in $\mathrm{c}+\mathrm{sc}$; radular spinules lacking. Legs including tarsal segments, yellow, femur brown. Meracanthus short, curved laterally, rounded apically. Abdomen brown, genitalia yellow. Male paramere long and slender, widest basally, tapering apically and directed posteriorly, few short setae posteriorly visible from lateral view. Measurements and ratios as in table $2 \mathrm{~A}-\mathrm{C}$.

Fifth instar larva: colour unknown. Antenna 3-segmented; segment 1 without a sectaseta, segment 2 with one sectaseta and segment 3 with five sectasetae grouped in 2, 2 and 1. Dorsal sclerites covered by few sectasetae smaller than marginal ones and few lanceolate setae. Forewing pad with 11-13 sectasetae marginally; humeral lobe present. Caudal plate rounded marginally; sectasetae present marginally, few or lacking dorsally with few lanceolate setae. Arolium fan-shaped and petiolate, indistinctly expanded basally. Anus terminal, outer circumanal ring with a single row of pores. Measurements and ratios as in table 3.

Host plants. The material from Palawan has been collected on ? Zyzygium sp. (Myrtaceae) which seems an unlikely host.

Distribution. Philippines (Mindanao) (Crawford, 1917, 1919) (Palawan) and Malaysia (Sarawak).

Material examined. Philippines: Lectotype $q$ and paralectotype $q$, Mindanao,

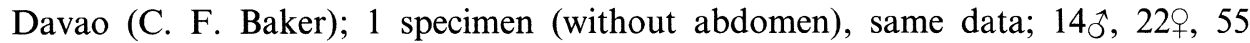
larvae, Palawan, Inagauan river, $700 \mathrm{~m}, 31$ January 1988, ? Zyzygium sp., ant attended (Pheidole longipes) (J. H. Martin). Malaysia: 1\%, Sarawak, 9 km SW Kapit, Sebadai Park, 50 m, 20 May 1994 (I. Löbl and D. Burckhardt).

Comments. The material from Palawan and Sarawak is only tentatively identified as $P$. brevicephala (Crawford). It differs from other species of the brevicephala type in the presence of a basal seta on the last antennal segment in adults. As the antennae are missing in the syntypes of $P$. brevicephala, the conspecificity of the two remains doubtful. The syntypes have darker forewings with dark brown apices of veins. There is, on the other hand, a good fit of the forewing shape between the two. For this uncertainty it is important to stabilize the name brevicephala for which reason a lectotype is designated.

\section{Paurocephala calcarata sp. $\mathrm{n}$. (figures 13D, 14D, G, 15D, J, 16D)}

Description. Species of the wilderi type.

Adult: head light brown; vertex covered by inconspicuous setae. Clypeus yellow. Antenna yellow, distal half of segment 4 and entire segments 6-10 brown; segment 9 without a long basal seta. Thorax brown and covered by inconspicuous setae dorsally, yellow to light brown laterally and ventrally. Metascutellar horn small, blunt apically. Forewing including pterostigma transparent; veins yellow. Forewing with inconspicuous setae on veins (vein Rs with 12-15 setae); Rs vein marginally curved throughout. Surface spinules confined to cell $\mathrm{cu}_{1}$ and apex of $\mathrm{m}_{1}$ and $\mathrm{m}_{2}$ of forewing; radular spinules present in $\mathrm{m}_{2}$, reduced in $\mathrm{cu}_{1}$. Legs white to yellow, tarsal segments brown. Meracanthus long, straight laterally, rounded apically. Abdomen including genitalia, yellow, tergites brown. Male paramere short, widest in the middle, rounded apically, few short setae posteriorly visible from lateral view; inner surface with two hooks apically, stout setae confined posteriorly in basal half. Measurements and ratios as in table $2 \mathrm{~A}-\mathrm{C}$.

Fifth instar larva: unknown. 
Host plants. Adults were collected on Ficus sp. (Moraceae) which may be the host. Distribution. Fiji Islands.

Material examined. Holotype ô, Fiji: Viti Levu, Lautoka, 23 October 1921, Ficus sp. (W. Greenwood) (BMNH). Dry mounted.

PARATYPES. Fiji: $6 \hat{0}, 2$, same data as holotype (BMNH, NHMB). Dry and slide mounted.

Comments. Similar to $P$. similis sp. $\mathrm{n}$. from which it differs in the paramere shape and the oval female circumanal ring.

\section{Paurocephala chonchaiensis Boselli}

(figures 12B, D, F, H, J)

Paurocephala chonchaiensis Boselli, 1929: 252; Lectotype ${ }_{0}^{*}$, (UNEI) examined, by present designation.

Paurocephala conchaiensis Boselli; Boselli, 1929: 259; 1930: 186; Hodkinson, 1983: 355. misspelling.

Paurocephala (Thoracocorna) chonchaiensis Boselli; Klimaszewski, 1970: 426; 1973: 161.

Paurocephala pumilae Li and Yang, 1987: 46. Holotype ô (BAUC), examined, syn. n.

Paurocephala zhejiangensis Yang and Li, 1987: 48. Holotype ô (BAUC), examined, syn. $\mathrm{n}$.

Description. Species of the chonchaiensis type.

Adult: similar to $P$. bifasciata differing in the overall darker body coloration; in the longer setae on forewing veins; surface spinules in cells of forewing denser; ratio of antennal length to head width and forewing length to forewing width. Measurements and ratios as in table $2 \mathrm{~A}-\mathrm{C}$.

Fifth instar larva: similar to $P$. bifasciata differing in coloration. Antenna yellow, distal half of flagellum brown. Dorsal sclerites brown. Caudal plate yellow to light brown. Measurements and ratios as in table 3.

Host plants. Ficus pumila L. (Moraceae).

Distribution. China (Yang and Li, 1987) (Hong Kong) and Japan (Miyatake, 1965).

Material examined. China: Lectotype $\hat{\jmath}$, paralectotypes $2 \hat{\jmath}, 3 \hat{q}, 5$ larvae, Fu-Chow, 27 November 1924, Ficus sp. (F. Silvestri); Holotype (of P. pumilae) ô, paratypes $7 \hat{0}, 69$, Guangdong, Hainan Island, Xinglong, Wenquan, 20-21 December 1974, Ficus pumila; Holotype (of P. zhejiangensis) ô, Zhejiang, Lin'an Co., Tianmushan, 4 May 1980; 49 ${ }^{\star}, 499,33$ larvae, Hong Kong, Hong Kong Island, Hill above Belchers, $200 \mathrm{~m}, 12-13$ November 1996, Ficus pumila (J. H. Martin). Japan: 3ㅅ, Kyushu, Hirao, Fukuoka, 10 June 1958, Ficus pumila (Y. Miyatake); 10ิ. Tsushima Is., Mitaka, Hagasaki, 30 August 1991 (K. Matsumoto) (BAUC, BMNH, MHNG, NHMB, OMNH, TULE). Dry and slide mounted and preserved in alcohol.

Comments. Some of the literature records of $P$. chonchaiensis Boselli concern $P$. bifasciata Kuwayama (see comments under $P$. bifasciata). To avoid future taxonomic confusion a lectotype is designated here for $P$. chonchaiensis Boselli.

Paurocephala curvata sp. $\mathrm{n}$.

(figures 13C, 14C, H, 15C, I, M, 16C, 38)

Description. Species of the wilderi type.

Adult: head and clypeus brown; vertex covered by short setae. Antenna yellow, apical half of segments 6 and 8 and entire segments $8-10$ brown; segment 9 without a long basal seta. Thorax entirely brown and covered by short setae dorsally. 
Metascutellar horn small, blunt apically. Forewing transparent; veins yellow to brown; pterostigma white, often with a central transparent area. Forewing with inconspicuous setae on veins (vein Rs with 17-19 setae); Rs vein marginally curved throughout. Surface spinules confined to cell $\mathrm{cu}_{1}$ and apex of $\mathrm{m}_{1}$ and $\mathrm{m}_{2}$ of forewing; radular spinules present in $\mathrm{m}_{2}$, reduced in $\mathrm{cu}_{1}$. Legs white, tarsal segments yellow to brown. Meracanthus long, straight laterally, rounded apically. Abdomen including genitalia, brown. Male paramere widest in the middle, tapering in apical third and curved anteriorly, few short setae posteriorly visible from lateral view; inner surface without stout setae. Measurements and ratios as in table 2A-C.

Fifth instar larva: colour unknown. Antenna 3-segmented, flagellum indistinctly subdivided; segments 1 without a sectaseta, segment 2 with one sectaseta, flagellum with five sectasetae grouped in 2,2 and 1. Dorsal sclerites covered by sectasetae and simple setae as large as marginal ones. Forewing pad with 7-10 sectasetae and six to eight simple setae marginally; humeral lobe lacking. Caudal plate rounded marginally; sectasetae present dorsally and marginally including long simple setae. Arolium fan-shaped, indistinctly expanded basally. Anus ventral, outer circumanal ring with a single row of pores, laterally multiporous. Measurements and ratios as in table 3.

Host plants. Unknown.

Distribution. Papua New Guinea and Indonesia (Sulawesi).

Material examined. Holotype ô, Papua New Guinea: Morobe, Buso, 14 November 1979 (J. H. Martin) (BMNH). Dry mounted.

Paratypes. Papua New Guinea: $7 \hat{\delta}, 5 q$ and 22 larvae, same data as holotype.

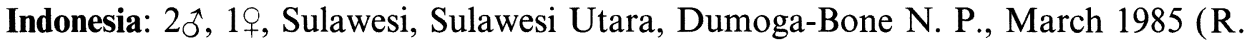
Ent. Soc. Lond., Project Wallace); 1ð̊, same data but $230 \mathrm{~m}, 2$ December 1985 (BMNH, NHMB). Dry and slide mounted.

Comments. $P$. curvata sp. $\mathrm{n}$. is distinguished from the other species of the wilderi type by the apically anteriorly curved paramere without stout setae on inner surface.

Paurocephala dayak sp. n.

(figures 21A, G, 23A, 24D, 25A, 26A, 28A)

Description. Species of the psylloptera type.

Adult: head brown with diffused yellow markings; vertex covered by long setae. Clypeus brown. Antenna brown, segments 1 and 2 and basal half of segment 3 yellow; segment 9 with a long seta in basal quarter. Thorax brown and covered by long setae dorsally, yellow to orange brown laterally and ventrally. Metascutellar horn small, pointed apically. Forewing transparent to light brown; veins yellow to light brown, apically brown to forewing margin; pterostigma white. Forewing with long setae on veins (vein Rs with 12-18 setae); Rs vein marginally curved in apical third. Surface spinules present in all cells of forewing; radular spinules present in $\mathrm{m}_{2}$, reduced in $\mathrm{cu}_{1}$. Legs white to yellow, tarsal segments dark brown. Meracanthus long, curved laterally, pointed apically. Abdomen including genitalia, orange to brown, sternites white to light brown. Male paramere long, almost straight posteriorly, widest in the middle and tapering apically, numerous long setae posteriorly visible from lateral view; inner surface with two hooks apically, one to four stout setae in basal fifth. Measurements and ratios as in table $2 \mathrm{~A}-\mathrm{C}$.

Fifth instar larva: colour unknown. Antenna 3-segmented, flagellum indistinctly subdivided; segment 1 without a sectaseta, segment 2 with one sectaseta, flagellum with five sectasetae grouped in 2,2 and 1. Dorsal sclerites covered by sectasetae as 
large as marginal ones, with few inconspicuous simple setae. Forewing pad with 8-10 sectasetae and one to three inconspicuous simple setae marginally; humeral lobe lacking. Caudal plate with shallow tubercle-like extensions marginally, apical excavation concave; sectasetae present dorsally and marginally with few inconspicuous simple setae. Arolium fan-shaped, indistinctly expanded basally. Anus ventral, outer circumanal ring with a single row of pores. Measurements and ratios as in table 3.

Host plants. Trema tomentosa (Roxb.) Hara and T. orientalis (L.) Blume (Ulmaceae).

Distribution. Malaysia (Sabah and Sarawak), Brunei and Singapore.

Material examined. HolotyPE ô, Malaysia: Sabah, Crocker range, Kimanis road, mile 10, 1150 m, 13 May 1982, Trema orientalis (D. Burckhardt) (MHNG). Dry mounted.

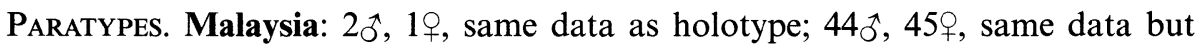
Batu Putih Cave, 9 May 1982, Trema? tormentosa (D. Burckhardt); $\widehat{\jmath}, 2$, Sarawak, Santubong, 32 km N, Kuching, 0-50 m, 28-29 May 1994, Trema cannabina (I. Löbl and D. Burckhardt); $12 \hat{\jmath}, 18 \%$ and 4 larvae, same data but Rumah Tuan, $13 \mathrm{~km} \mathrm{E}$ Kapit, 50 m, 20 May 1994, Trema tomentosa 13 1 , 5ㅇ and 1 larval exuvia, same data but Sebadai Park, 9 km SW Kapit, 2ð, 9 9 , same data but Gunong Mulu N. P., Base Camp Helipad, RGS Mulu exp., Trema sp. (D. Hollis); Brunei: 4ㅅ, 7ㅇ, Penanjong,

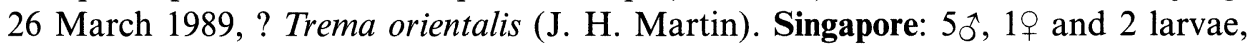
Botanic Gardens forest, Napier road, 2 March 1994, Trema sp. vigourosly ant attended (J. H. Martin) (BAUC, BMNH, MHNG, NHMB). Dry and slide mounted and preserved in alcohol.

Comments. Similar to $P$. setifera (Crawford) from which it differs in the adult by the presence of a basal seta on antennal segment 9 . No morphological characters could be found to separate the larvae of $P$. dayak from $P$. setifera, $P$. palawanensis sp. n. and $P$. polaszeki.

Paurocephala distincta sp. $\mathrm{n}$.

(figures $17 \mathrm{~F}, 18 \mathrm{~F}, 19 \mathrm{~F}, \mathrm{O}, 20 \mathrm{~F}$ )

Description. Species of the psylloptera type.

Adult: head brown, yellow towards lateral ocellus and along coronal suture; vertex covered by inconspicuous setae. Clypeus yellow. Antenna brown, segments 1-3 yellow; segment 9 with a long seta in basal quarter. Thorax brown with yellow markings along body axis and covered by short setae dorsally, yellow to brown laterally and ventrally. Metascutellar horn small, pointed apically and directed posteriorly. Forewing transparent to white; veins light brown; pterostigma white. Forewing with inconspicuous setae on veins (vein Rs with 9-13 setae); Rs vein straight. Surface spinules present in all cells of forewing; radular spinules present in $\mathrm{m}_{2}$, reduced in $\mathrm{cu}_{1}$. Legs yellow to brown, tarsal segments dark brown. Meracanthus long, straight laterally, pointed apically. Abdomen, including genitalia, yellow, tergites brown. Male paramere widest basally, tapering towards apex, few long setae posteriorly visible from lateral view; inner surface with one hook apically, one or two stout setae at base. Measurements and ratios as in table $2 \mathrm{~A}-\mathrm{C}$.

Fifth instar larva: unknown.

Host plants. Adults were collected on Ficus sp. (Moraceae) which could be the host. 
Distribution. India and Sri Lanka.

Material examined. Holotype ô, India: Karnataka, Mudigere, 5 January 1989, Ficus sp. (C. Parvathi) (BMNH). Dry mounted.

PARATYPES. India: $1 \hat{\jmath}, 2$, same data as holotype. Sri Lanka: 1ㅇ, 1961/62 (E. Judenko) (BMNH, NHMB). Dry and slide mounted.

Comments. P. distincta sp. $\mathrm{n}$. is similar to P. psylloptera Crawford from which it differs in the shape of the pterostigma.

Paurocephala elegans sp. $\mathrm{n}$.

(figures 9A, F, 10A, C, E, G)

Description. Species of the kleinhofiae type.

Adult: head orange, sides along coronal suture and area around median ocellus white; vertex covered by inconspicuous setae. Clypeus orange. Antenna yellow, segments 3, 7 and 8 brown; segment 7 or 8 without a long basal seta. Thorax orange and covered by inconspicuous setae dorsally, yellow to white laterally and ventrally. Metascutellar horn large, pointed apically and directed posteriorly. Forewing transparent; veins yellow, apically brown to forewing margin; pterostigma white, basally and apically brown. Forewing with inconspicuous setae on veins (vein Rs with 12-14 setae); Rs vein strongly curved in basal third. Surface spinules and radular spinules lacking. Legs yellow, last tarsal segment brown. Meracanthus short, straight laterally, rounded apically. Tergites orange brown, sternites light brown, genitalia yellow to brown. Male paramere elongate, almost straight posteriorly, widest in the middle, tapering apically, few short setae posteriorly visible from lateral view. Measurements and ratios as in table $2 \mathrm{~A}-\mathrm{C}$.

Fifth instar larva: unknown.

Host plants. Unknown.

Distribution. Indonesia (Sulawesi).

Material examined. Holotype $\hat{0}$, Indonesia: Sulawesi, Sulawesi Utara, DumogaBone N. P., March 1985 (R. Ent. Soc. Lond., Project Wallace) (BMNH). Dry mounted.

PARATYPES. Indonesia: $3 \hat{0}, 69$, same data as holotype; 1 , same data but $230 \mathrm{~m}$, 2 December 1985; 1ิ, 19, same data but 11 and 19 July 1985 (BMNH, NHMB).

Comments. P. elegans sp. $\mathrm{n}$. is distinguished from the other species of the kleinhofiae type by the very long apical setae on the antennae (longer than flagellum) and the large metascutellar horn.

\section{Paurocephala gossypii Russell}

(figures 5B, F, 8H, 36A)

Paurocephala gossypii Russell, 1943: 115. Holotype $\widehat{\jmath}$, examined.

Paurocephala gossypii Russell; Vondráček, 1963: 278.

Description. Species of the gossypii type.

Adult: described by Russell (1943). Measurements and ratios as in table 2A-C.

Fifth instar larva: described by Russell (1943). Measurements and ratios as in table 3.

Host plants. Gossypium spp. (Malvaceae).

Distribution. Zaire (Russell, 1943; Vondráček, 1963), Sudan and Malawi.

Material examined. Zaire: Holotype ô, Gandajika, November 1941, Gossypium sp. (D. Soyer) (USNM); paratypes, 93ㅅ, 84우 and numerous larvae, same data as 
holotype; 19, Nyasaland, 23 March 1922, Gossypium sp. (C. Smee); 4수, 2q, 6 larvae, Murumba, 29 May 1963; 3ㅇ, Kabemba, 22 June 1918 (R. Mayné). Sudan: 1수 1우, Western Equatoria, Maridi, 18 November 1962 (H. R. Yust); 50, 1, 30 larvae, Yambio, 9 November 1962, Gossypium sp. (W. T. C. Higgins); 90, 49, 5 larvae, same data (no collecting date) but Gezira. Malawi: $6 \hat{\phi}, 12+$, Makoka, 19 February 1970, Gossypium sp. (M. D. Mowlam); 10^, 2q, Chitala, May 1964, Gossypium sp. (D. J. McKinley); $1 \hat{\sigma}, 3$, , no data (K. Sattler) (BMNH, MRAC, NHMB, USNM). Dry and slide mounted.

Comments. P. gossypii Russell is distinguished from both $P$. abutili sp. n. and $P$. urenae Russell by the shape of the antenna; antennal segments shorter and wider; segment four quadrate. The fifth instar larva is distinguished by the smaller number of sectasetae on dorsum of body.

\section{Paurocephala hollisi sp. $\mathrm{n}$. \\ (figures 6A, F, 7A, D, G, J, 8J)}

Description. Species of the gossypii type.

Adult: head brown, yellow towards lateral ocelli; vertex covered by short setae. Clypeus brown. Antenna yellow, segments 4, 6 and 8-10 brown; segment 9 without a long basal seta. Thorax entirely brown and covered by inconspicuous setae dorsally. Metascutellar horn small, hump-shaped. Forewing including pterostigma brown; veins dark brown. Forewing with short setae on veins (vein Rs with 27-30 setae); Rs vein strongly curved at distal third. Surface spinules present in all cells of forewing; radular spinules lacking. Legs yellow to brown, tarsal segments darker. Meracanthus short, laterally straight, rounded apically. Abdomen, including genitalia, brown. Male paramere widest in basal third, tapering apically and directed posteriorly, few short setae posteriorly visible from lateral view; inner surface with a row of six or seven peg-like setae situated between basal fifth and apical third. Measurements and ratios as in table $2 \mathrm{~A}-\mathrm{C}$.

Fifth instar larva: unknown.

Host plants. Adults were collected on Agelaea heterophylla Gilg. (Connaraceae) which seems an unlikely host.

Distribution. Tanzania.

Material examined. Holotype $\hat{\alpha}$, Tanzania: E Usambara Mountains, Amani (research station), ca $3000 \mathrm{ft}, 19-27$ June 1974, Agelaea heterophylla (D. Hollis) (BMNH). Dry mounted.

ParaTYPeS. Tanzania: $17 \delta^{\wedge}, 14$, same data as holotype (BMNH, NHMB). Dry and slide mounted.

Comments. Distinct from the other species of the gossypii type by the dark body coloration. It is most similar to $P$. sinuata sp. $\mathrm{n}$. from which it is distinguished by the smaller body dimensions.

\section{Paurocephala insolita sp. $\mathrm{n}$.}

(figures 6E, J, 7C, F, I, L, 8L, 35)

Description. Species of the gossypii type.

Adult: head and clypeus white; vertex covered by short setae. Antenna white, apical half of segments 4, 6 and 8 and entire segments 9 and 10 brown; segment 9 without a long basal seta. Thorax white to light brown and covered by inconspicuous setae dorsally, laterally and ventrally white. Metascutellar horn small, blunt apically. 
Forewing whitish; veins light yellow, apically brown to forewing margin, central part of veins $\mathrm{A}, \mathrm{R}+\mathrm{M}+\mathrm{Cu}_{1}, \mathrm{R}$ and $\mathrm{M}+\mathrm{Cu}_{1}$ brown, apical third of $\mathrm{Rs}$ vein brown; pterostigma brown basally and apically. Forewing with inconspicuous setae on veins (vein Rs with 12-16 setae); Rs vein marginally curved in distal third. Surface spinules present in all cells of forewing; radular spinules lacking. Legs including tarsal segments, white to yellow. Meracanthus short, almost straight laterally, rounded apically. Abdominal tergites light brown, sternites white, genitalia brown. Male paramere almost straight posteriorly, widest in the middle and tapering apically, few long setae in basal half posteriorly visible from lateral view. Measurements and ratios as in table $2 \mathrm{~A}-\mathrm{C}$.

Fifth instar larva: antenna yellow, base and apical third of flagellum brown; 3 -segmented, segment 1 without a sectaseta, segment 2 with one sectasetae and segment 3 with five sectasetae grouped in 2, 2 and 1. Dorsal sclerites brown, covered by sectasetae as large as marginal ones and few inconspicuous simple setae. Wing pads yellow, brown apically and basally; forewing pad with five to seven sectasetae and one to three inconspicuous simple setae marginally; humeral lobe lacking. Caudal plate yellow to light brown, marginally darker, with large tubercle-like extensions marginally, ending in a rounded process, V-shaped excavated apically; sectasetae present dorsally and marginally with few inconspicuous simple setae. Arolium triangular and petiolate, expanded basally. Anus ventral, outer circumanal ring with a single row of pores. Measurements and ratios as in table 3.

Host plants. Psorospermum febrifugum Spach (Clusiaceae).

Distribution. Angola.

Material examined. Holotype ô, Angola: Gabela, 18 September 1971, Psorospermum febrifugum (A. van Harten) (BMNH). Dry mounted.

PARATYPES. Angola: $80^{*}, 21 \%$ and 16 larvae, same data as holotype (BMNH, NHMB). Dry and slide mounted and preserved in alcohol.

Comments. Similar to $P$. lienhardi from which it differs in the absence of bifid setae on the antennae and in the shape of the male paramere. $P$. insolita is the only member of Paurocephala whose host plant is outside the Malvales.

\section{Paurocephala javanica sp. $\mathrm{n}$.}

(figures 13E, 14E, 15E, K, 16E, 40)

Description. Species of the wilderi type.

Adult: head orange to brown; vertex covered by long setae. Clypeus yellow to light brown. Antenna brown, segments 1-3 yellow; segment 9 without a basal long seta. Thorax orange to brown and covered by long setae dorsally, brown laterally and ventrally. Metascutellar horn small, blunt apically. Forewing transparent to light brown; veins brown; pterostigma white. Forewing with long setae on veins (vein Rs with 16-19 setae); Rs vein curved throughout. Surface spinules present in all cells of forewing, reduced in $\mathrm{c}+\mathrm{sc}$; radular spinules present in $\mathrm{m}_{2}$, reduced in $\mathrm{cu}_{1}$. Legs yellow to brown, tarsal segments darker. Meracanthus long, straight laterally, rounded apically. Abdomen including genitalia, brown. Male paramere long, almost straight posteriorly, widest in the middle and tapering towards apex, few short setae posteriorly visible from lateral view; inner surface with a row of long stout setae confined posteriorly in basal third. Measurements and ratios as in table $2 \mathrm{~A}-\mathrm{C}$.

Fifth instar larva: colour unknown. Antenna 3-segmented, flagellum indistinctly 
subdivided; segments 1 and 2 without sectasetae, flagellum with five sectasetae grouped in 2, 2 and 1. Dorsal sclerites covered by sectasetae as large as marginal ones and few short simple setae. Forewing pad with six to eight sectasetae and one or two short simple setae marginally; humeral lobe lacking. Caudal plate rounded marginally; sectasetae present dorsally and marginally with a few short simple setae. Arolium triangular and petiolate, expanded basally. Anus terminal, outer circumanal ring with one or two rows of pores, subdivided at irregular intervals. Measurements and ratios as in table 3 .

Host plants. Ficus sp. (Moraceae).

Distribution. Indonesia (Java).

Material examined. HolotyPe ơ, Indonesia: Java, Bogor, November 1986, Ficus sp., ant tended (Camponotus sp.) (M. R. Wilson) (BMNH). Dry mounted.

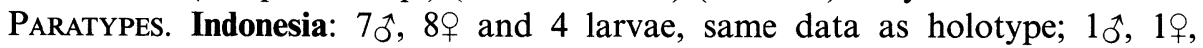
Cibulan, ca 700 m, 16 April 1959 (B. Pisarski and J. Proszyriski) (BMNH, NHMB). Dry and slide mounted.

Comments. $P$. javanica sp. $\mathrm{n}$. is distinguished from other species of the wilderi type by the transluscent forewings.

\section{Paurocephala kleinhofiae Uichanco, comb. rev.} (figures 9B, C, H, I, G, 10B, D, F, H)

Paurocephala kleinhofiae Uichanco, 1921: 274. Syntypes, 50ิ, 9요 Philippines: Luzon, Los Baños, 50 m, December 1918, Kleinhovia hospita (L. B. Uichanco) (UPPC), not examined. Anomoterga kleinhofiae (Uichanco); Navasero and Calilung, 1998: 14.

Description. Species of the kleinhofiae type.

Adult: head yellow, sides along coronal suture white; vertex covered by inconspicuous setae. Clypeus orange. Antenna yellow, segment 9 brown; segment 8 with a long basal seta. Thorax yellow and covered by inconspicuous setae dorsally, white laterally and ventrally. Metascutellar horn small, pointed apically. Forewing including pterostigma transparent; veins yellow, apically light brown to forewing margin. Forewing with inconspicuous setae on veins (vein Rs with 11-14 setae); Rs vein marginally curved throughout. Surface spinules absent or confined to cells in apical half of forewing; radular spinules present in $\mathrm{m}_{2}$, reduced or lacking in $\mathrm{cu}_{1}$. Legs including tarsal segments, yellow to white. Meracanthus short, straight laterally, rounded apically. Abdomen, including genitalia, yellow to brown. Male paramere elongate, widest in the middle and rounded apically, few short setae posteriorly visible from lateral view. Measurements and ratios as in table $2 \mathrm{~A}-\mathrm{C}$.

Fifth instar larva: described by Fang and Yang (1986).

Host plants. Kleinhovia hospita L. (Sterculiaceae).

Distribution. Philippines (Luzon) (Uichanco, 1921) (Mindanao) and Taiwan (Fang and Yang, 1986).

Material examined. Philippines: 1, Mindanao, Davao (experimental station), October 1961, (M. R. Gavarra); 1 q same data but September 1962 (BMNH). Taiwan: 2ર̂, 2ㅇ, Ping-tung, Ken-ting, 22 March 1985, Kleinhovia hospita (S. J. Fang) (BMNH, NHMB, NMNS). Dry and slide mounted.

Comments. Syntypes of Paurocephala kleinhofiae Uichanco were not available for study. Fang and Yang (1986) recorded the species from Taiwan. Two females from the Philippines (Mindanao), which differ in certain details from the material from Taiwan, were examined. They differ in the shape of the forewing veins (Rs 
vein more strongly arched) (figure 9B, C) and the setae which are associated with the antennal rhinaria. These setae are much longer (figure $9 \mathrm{H}$ ) than in the Philippine material. The material from Mindanao fits well with the description provided by Uichanco (1921) such as forewing and shape of Rs vein.

\section{Paurocephala lienhardi sp. $\mathrm{n}$.}

(figures 9D, 11A, C, F, H)

Description. Species of the gossypii type.

Adult: head white, dark brown anteriorly; vertex covered by long setae. Clypeus yellow. Antenna yellow; segment 9 without a long basal setae. Thorax brown and covered by long setae dorsally, yellow to brown laterally and ventrally. Metascutellar horn small, pointed apically. Forewing transparent; veins light yellow, vein A brown basally; basal half of pterostigma dark brown. Forewing with short setae on veins (vein Rs with 15-17 setae); Rs vein strongly curved in apical third. Surface spinules sparse, absent from cell $\mathrm{c}+\mathrm{sc}$; radular spinules lacking. Legs including tarsal segments, yellow to white, distal third of metafemur brown. Meracanthus short, straight laterally, rounded apically. Abdomen, including genitalia, yellow to brown. Male paramere short, widest apically, few short setae posteriorly visible from lateral view. Measurements and ratios as in table $2 \mathrm{~A}-\mathrm{C}$.

Fifth instar larva: unknown.

Host plants. Unknown.

Distribution. Malaysia (Sabah).

Material examined. HolOtyPE ô and PARATYPE + , Malaysia: Sabah, Kinabalu National Park, 1750-1850 m, 20 March 1983 (C. Lienhard) (MHNG). Slide mounted.

Comments. Similar to $P$. insolita sp. $\mathrm{n}$. from which it differs in the presence of bifid setae on the antenna and in the shape of the male paramere.

\section{Paurocephala lii sp. $\mathrm{n}$. \\ (figures 29A, D, G, 30A, D, G)}

Description. Species of the brevicephala type.

Adult: head yellow to orange, white along sides of coronal suture and towards genae; vertex covered by inconspicuous setae. Clypeus yellow. Antenna yellow to orange, segment 7 and 8 brown; segment 7 with a long basal seta. Thorax yellow orange to brown and covered by short setae dorsally, white laterally and ventrally. Metascutellar horn large, pointed apically. Forewing transparent to white; veins yellow, apically brown to forewing margin; pterostigma white, brown basally and apically. Forewing with inconspicuous setae on veins (vein Rs with 16-20 setae); Rs vein marginally curved in basal quarter. Surface spinules sparse, absent in cell c $+\mathrm{sc}$; radular spinules lacking. Legs including tarsal segments, white, foretarsi brown. Meracanthus short, almost straight laterally, rounded apically. Abdomen including genitalia, white, tergites orange to brown. Male paramere elongate, widest basally and tapering apically, directed posteriorly in apical half, few short setae posteriorly visible from lateral view. Measurements and ratios as in table $2 \mathrm{~A}-\mathrm{C}$.

Fifth instar larva: unknown.

Host plants. Unknown.

Distribution. Indonesia (Sulawesi).

Material examined. HolotyPe ô, Indonesia: Sulawesi, Sulawesi Utara, 
Dumoga-Bone N. P., 400 m, 11 February 1985 (fogging) (R. Ent. Soc. Lond., Project Wallace) (BMNH). Dry mounted.

PARATYPES. Indonesia: $4 \hat{\jmath}, 4$, , same data as holotype; $2 \hat{\jmath}, 19+$, same data but $230 \mathrm{~m}, 11$ July $1985 ; 1 \jmath$, 1 우, same data but $400 \mathrm{~m}$, 19 July $1985 ; 1 \jmath$, same data but

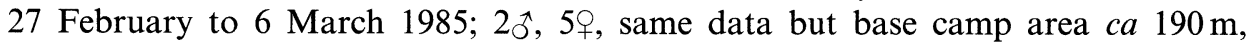
9-16 May 1985 (yellow pan traps) (J. S. Noyes); 2ર’, same data but 6-13 July 1985 and 30 October to 6 November 1985 (BMNH, NHMB). Dry and slide mounted.

Comments. This species is similar to $P$. minuta Crawford but since the type material of the latter is in very bad condition, no detailed comparison can be made. $P$. lii differs from $P$. minuta Crawford in the forewing shape.

\section{Paurocephala longiantennata sp. $\mathrm{n}$.}

(figures 17B, 18B, 19B, J, 20B)

Description. Species of the psylloptera type.

Adult: head brown with two small yellow spots on either side of vertex towards lateral ocelli; vertex covered by long setae. Clypeus brown. Antenna brown, segments 1-3 and basal half of segment 4 yellow; segment 9 with a long seta in basal quarter. Thorax brown (in females with yellow stripes along body axis) and covered by long setae dorsally, light to dark brown laterally and ventrally. Metascutellar horn small, pointed apically and directed posteriorly. Forewing transparent; veins brown; pterostigma brown. Forewing with short setae on veins (vein Rs with 17-20 setae); Rs vein strongly curved in the middle. Surface spinules confined to cells $\mathrm{cu}_{1}$, apex of $\mathrm{m}_{1}$ and $\mathrm{m}_{2}$; radular spinules present in $\mathrm{m}_{2}$, reduced in $\mathrm{cu}_{1}$. Legs yellow to brown, tarsal segments darker. Meracanthus long, curved laterally, pointed apically. Abdomen, including genitalia, brown. Male paramere long, apical fifth marginally directed posteriorly, widest basally and apically rounded, few short setae posteriorly visible from lateral view; inner surface with two hooks apically, a row of stout setae confined in basal two-thirds. Measurements and ratios as in table $2 \mathrm{~A}-\mathrm{C}$.

Fifth instar larva: unknown.

Host plants. Unknown.

Distribution. Papua New Guinea.

Material examined. Papua New Guinea: Holotype ô, Mafulu, $4000 \mathrm{ft}$, January 1934 (L. E. Cheesman) (BMNH). Dry mounted.

Paratype. Papua New Guinea: 1 $q$, same data as holotype; $3 \circ, 3 \circ$, NE New Guinea, Sakalang, Salawaket, September 1956 (E. J. Ford, Jr) (BMNH, BPBM, NHMB). Dry and slide mounted.

Comments. $P$. longiantennata sp. $\mathrm{n}$. is similar to $P$. maculipennis Uichanco from which is distinguished by the larger body dimensions, longer antennae and the larger number of stout setae on inner face of paramere.

Paurocephala lucida sp. $\mathrm{n}$.

(figures 5D, H, I)

Description. Species of the gossypii type.

Adult: head yellow; vertex covered by inconspicuous setae. Clypeus brown. Antenna yellow, apical half of segment 8 and entire segments 9 and 10 brown; segment 9 without a long basal seta. Thorax yellow and covered by inconspicuous setae dorsally, brown laterally and ventrally. Metascutellar horn small, blunt apically. Forewing transparent; veins yellow, apically light brown to forewing margin, central 
part of vein A brown; pterostigma yellow. Forewing with inconspicuous setae on veins (vein Rs with 23-25 setae); Rs vein strongly curved at apical third. Surface spinules sparse, present in distal parts of cells $\mathrm{m}_{1}, \mathrm{~m}_{2}$ and $\mathrm{cu}_{1}$ and throughout $\mathrm{cu}_{2}$; radular spinules lacking. Legs including tarsal segments, yellow to brown. Meracanthus short, laterally curved, rounded apically. Abdomen, including genitalia, brown. Male unknown. Measurements and ratios as in table 2A-C.

Fifth instar larva: unknown.

Host plants. The material was collected on flowers of Discopodium penninervium (Solanaceae), an unlikely host.

Distribution. Ethiopia.

Material examined. Holotype + and PARATYPe $q$, Ethiopia: Simien Mountains (W Mai Shaha valley), ca $9000 \mathrm{ft}, 14$ December 1952, flowers of Discopodium penninverium (Hugh Scott) (BMNH). Dry and slide mounted.

Comments. P. lucida sp. $\mathrm{n}$. is distinguished from the other species of the gossypii type by stout setae present on the first three visible abdominal tergites laterally.

Paurocephala macrochaetis sp. $\mathrm{n}$.

(figures 22A, 23F, 24I, 25F, 26F, 28F)

Description. Species of the psylloptera type.

Adult: head including clypeus brown; vertex covered by short setae. Antenna brown, segments 1 and 2 and basal half of segment 3 yellow; segment 9 with a long seta in basal quarter. Thorax brown and covered by inconspicous setae dorsally, yellow to light brown laterally and ventrally. Metascutellar horn small, pointed apically. Forewing transparent; veins light white to yellow; pterostigma white. Forewing with inconspicuous setae on veins (vein Rs with 7-10 setae); Rs vein marginally curved in apical third. Surface spinules confined to apex of cell $\mathrm{m}_{1}$; radular spinules present in $\mathrm{m}_{2}$, reduced in $\mathrm{cu}_{1}$. Legs white to yellow, last tarsal segment dark brown. Meracanthus long, marginally curved laterally, pointed apically. Abdomen including genitalia, white to light brown. Male paramere long, almost straight posteriorly, rounded apically, few long setae posteriorly visible from lateral view; inner surface with two hooks apically, one to three stout setae in basal fifth. Measurements and ratios as in table $2 \mathrm{~A}-\mathrm{C}$.

Fifth instar larva: unknown.

Host plants. Unknown.

Distribution. Indonesia (Sulawesi).

Material examined. Indonesia: Holotype ô, Sulawesi, Dumoga-Bone N. P., March 1985 (R. Ent. Soc. Lond., Project Wallace) (BMNH). Dry mounted.

PARATYPES. Indonesia: $4 \hat{\jmath}, 4$, same as holotype, Sulawesi, Dumoga-Bone N. P., March 1985; 1ml, same data as above but 11 July 1985, 230 m; 19, same data but 19 July 1985, 400 m. (BMNH). All material was collected by fogging.

Comments. Similar to P. sauteri (Enderlein) from which it differs in the smaller body dimensions and the much longer terminal setae on antennal segment 10 .

Paurocephala maculipennis Uichanco, stat. n.

(figures 17A, 18A, G, 19A, I, K, 20A, 42)

Paurocephala psylloptera maculipennis Uichanco, 1921: 276. Syntypes, 14^^, 8 , Philippines: Luzon, Laguna, Los Baños, 2 February 1918, Ficus nota (C. F. Baker) Acc. No. 18178 (UPPC), not examined. 
Description. Species of the psylloptera type.

Adult: head and clypeus brown; vertex covered by inconspicuous setae. Antenna brown, segments 1-3, basal half of segment 4, and entire segments 5 and 6 yellow; segment 9 without a long basal seta. Thorax entirely brown and covered by short setae dorsally. Metascutellar horn small, blunt apically. Forewing transparent; veins brown; pterostigma brown. Forewing with short setae on veins (vein Rs with 11-15 setae); Rs vein strongly curved in the middle. Surface spinules confined to cells $\mathrm{cu}_{1}$, apex of $\mathrm{m}_{1}$ and $\mathrm{m}_{2}$; radular spinules present in $\mathrm{m}_{2}$, reduced in $\mathrm{cu}_{1}$. Legs yellow to brown, tarsal segments darker. Meracanthus short, curved laterally, pointed apically. Abdomen brown, genitalia yellow. Male paramere almost straight, widest in distal third, truncate apically, few relatively short setae posteriorly visible from lateral view; inner surface with two hooks apically, row of stout setae confined to basal half. Measurements and ratios as in table $2 \mathrm{~A}-\mathrm{C}$.

Fifth instar larva: colour unknown. Antenna 9-segmented; segments 1 and 2 without sectaseta, flagellum with five sectasetae grouped in 2, 2 and 1. Dorsal sclerites covered by sectasetae as large as marginal ones, simple setae lacking or inconspicuous. Forewing pad with four or five sectasetae marginally; humeral lobe lacking. Caudal plate rounded marginally; sectasetae present dorsally and marginally. Arolium triangular and petiolate, expanded basally. Anus ventral, outer circumanal ring with a single row of pores, laterally connected to two circular pore fields. Measurements and ratios as in table 3.

Host plants. Ficus nota (Moraceae).

Distribution. Philippines (Uichanco, 1921; Braza and Calilung, 1981) including Palawan (Miyatake, 1971 p.p.)

Material examined. Philippines: 20ิ, 1q, Maao, Occ. Negros, P. I., 27 December 1929, 'Locotol', Mailum forest (W. D. Pierce); 1ðَ, 1ㅇ, Los Baños, Mt Makiling, 3 December 1988, Ficus sp. (A. T. Barrion); 6수 5ㅇ, 3 larval exuviae, same data but 9 April 1965, Ficus nota (L. D. Tuthill); 4^^, 3ㅇ, same data but Laguna, College, 16 April 1976 (R. D. Braza); 19, Davao (experimental station), May 1962 (M. R.

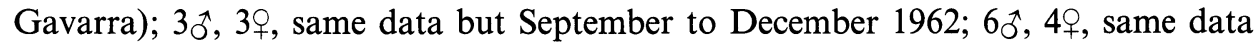

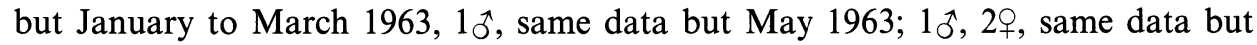
November to December 1963, 3ㅇ, same data but January to March 1964; Palawan: 4રิ, 1ㅇ, 18 larvae, Mt St Paul N. P., Sabang Cabayugan road, 25 January 1988, (?) Ficus sp., ant-attended (J. H. Martin) (BMNH, NHMB, USNM). Dry and slide mounted.

Comments. P. maculipennis Uichanco differs from $P$. psylloptera Crawford in the shape of vein Rs in the forewing and in the shape of the genitalia. It is considered to be a species distinct from $P$. psylloptera and given here species status.

Paurocephala marginata sp. $\mathrm{n}$.

(figures 21C, 23C, 24F, 25C, 26C, 28C)

Description. Species of the psylloptera type.

Adult: head brown; vertex covered by long setae. Clypeus brown. Antenna brown, segments 1, 2 and basal third of segment 3 yellow; segment 9 without a long basal seta. Thorax brown with yellow markings along body axis and covered by long setae dorsally, light to dark brown laterally and ventrally. Metascutellar horn small, pointed apically. Forewing white; veins yellow to light brown, distinctly brown to forewing margin (apical third of vein Rs, entire veins $\mathbf{M}_{1+2}$ and $\mathbf{M}_{3+4}$, and apical 
third of vein $\mathrm{Cu}_{1 \mathrm{a}}$ ); pterostigma yellow to white. Forewing with long setae on veins (vein Rs with 18-22 setae); Rs vein marginally curved in apical third. Surface spinules present in all cells of forewing; radular spinules present in $\mathrm{m}_{2}$ and $\mathrm{cu}_{1}$. Legs white to yellow, tarsal segments brown. Meracanthus long, curved laterally, pointed apically. Abdomen including genitalia, brown. Male paramere long, widest at basal third, almost straight posteriorly, rounded apically, long setae posteriorly visible from lateral view; inner surface with two hooks apically, four to seven stout setae in basal fifth. Measurements and ratios as in table $2 \mathrm{~A}-\mathrm{C}$.

Fifth instar larva: unknown.

Host plants. Adults were collected on Trema orientalis (L.) Blume (Ulmaceae) which could be the host.

Distribution. Indonesia (Sulawesi).

Material examined. HolotyPE $\mathcal{o}^{\wedge}$, Indonesia: Sulawesi, Dumoga-Bone N. P. (base camp area), ca 190 m, 9-16 May 1985, Trema ? orientalis (J. S. Noyes) (BMNH). Dry mounted.

PARATYPES. Indonesia: $1 \hat{\jmath}$, same data as holotype; $3 \hat{\jmath}, 3$, , Danau Mooat, 18 June 1985, 1080 m, Trema ? orientalis (J. H. Martin); 19, same data but Mogonipa, $400 \mathrm{~m}$, 13 June 1985 (BMNH, NHMB). Dry and slide mounted.

Comments. Paurocephala marginata sp. $\mathrm{n}$. is distinguished from the other species of the psylloptera type by the large body dimentions and the brown pattern at end of veins to forewing margin.

\section{Paurocephala martini sp. $\mathrm{n}$.}

(figures 31B, 32E, K, 33B, H, 34B)

Description. Species of the brevicephala type.

Adult: head light brown, darker towards lateral ocelli, yellow towards genae; vertex covered by long setae. Clypeus yellow. Antenna yellow, segment 8 brown; segment 7 with a long basal seta. Thorax orange to brown and covered by short setae dorsally, brown laterally and ventrally. Metascutellar horn small, wide basally, blunt apically. Forewing transparent; veins light yellow, apically brown to forewing margin, central part of vein A brown; pterostigma white. Forewing with long setae on veins (vein Rs with 12-17 setae); Rs vein marginally curved in apical half. Surface spinules spare, absent in cell $\mathrm{c}+\mathrm{sc}$; radular spinules lacking. Legs including tarsal segments, yellow. Meracanthus short, almost straight laterally, rounded apically. Abdominal tergites red to brown, sternites white, genitalia yellow to orange. Male paramere elongate, widest basally, tapering apically, apical half directed posteriorly, few short setae posteriorly visible from lateral view. Measurements and ratios as in table 2A-C.

Fifth instar larva: unknown.

Host plants. Unknown.

Distribution. Singapore.

Material examined. Holotype ô, Singapore: Bukit Timah Nature Reserve, 3 April 1989 (J. H. Martin) (BMNH). Dry mounted.

PARATYPES. Singapore: $5 \hat{\jmath}, 4+$, same data as holotype; $3 \hat{\jmath}, 9+$, same data but 30 December 1996, ? Ulmaceae (BMNH, MHNG, NHMB). Dry and slide mounted.

Comments. P. martini sp. $\mathrm{n}$. is distinguished from the other members of the brevicephala type by the reduced surface spinules on forewing cells (absent in cell $\mathrm{c}+\mathrm{sc}$ ) and the long bifid setae on antennal rhinaria. 
Paurocephala mathuri sp. $\mathrm{n}$.

(figures 29B, E, H, 30B, E, H)

Paurocephala nr. minuta Crawford; sensu Mathur, 1975: 53.

Description. Species of the brevicephala type.

Adult: head brown, yellow towards genae; vertex covered by inconspicuous setae. Clypeus orange. Antenna yellow to orange, segments 7 and 8 brown; segment 7 and 8 without a long basal seta. Thorax orange to brown and covered by inconspicuous setae dorsally, yellow to brown laterally and ventrally. Metascutellar horn large, pointed apically. Forewing transparent to light yellow; veins yellow, apically dark brown to forewing margin, central part of vein A brown; pterostigma transparent, brown apically and basally. Forewing with inconspicuous setae on veins (vein Rs with 9-12 setae); Rs vein strongly curved in the middle. Surface spinules and radular spinules lacking in forewing. Legs, including tarsal segments, yellow to white. Meracanthus short, almost straight laterally, rounded apically. Abdomen, including genitalia, brown. Male paramere elongate, widest basally, tapering apically, distal third directed posteriorly, few short setae posteriorly visible from lateral view. Measurements and ratios as in table $2 \mathrm{~A}-\mathrm{C}$.

Fifth instar larva: described by Mathur (1975).

Host plants. Kydia calycina Roxb. (Malvaceae).

Distribution. India (Uttar Pradesh) (Mathur, 1975).

Material examined. HolotyPE $\hat{0}$, India: Uttar Pradesh, Dehra Dun, New Forest, 11 April 1950, Kydia calycina (R. N. Mathur) (USNM). Dry mounted.

PARATYPES. India: $1 \hat{\delta}, 2 \circ$, same data as holotype (NHMB, USNM). Dry and slide mounted.

Comments. Similar to P. stephaniella sp. n. from which it differs in the absence of an expanded forewing pattern and the shape of the genitalia.

\section{Paurocephala medleri sp. $\mathrm{n}$.}

(figures 6D, I, 8B, D, F, N)

Description. Species of the gossypii type.

Adult: head orange; vertex covered by inconspicuous setae. Clypeus yellow. Antenna yellow, apical third of segment 8 and entire segments 9 and 10 brown; segment 9 without a long basal seta. Thorax orange and covered by inconspicuous setae dorsally, yellow laterally and ventrally. Metascutellar horn small, blunt apically. Forewing including pterostigma transparent; veins yellow to brown, apically light brown to forewing margin, central part of vein A brown. Forewing with inconspicuous setae on veins (vein Rs with 10-12 setae); Rs vein marginally curved in apical third. Surface spinules sparse, confined to cells in apical half of forewing and in cell $\mathrm{Cu}_{2}$; radular spinules lacking. Legs including tarsal segments, yellow. Meracanthus small, curved laterally, rounded apically. Abdomen, including genitalia, yellow to orange. Male paramere straight posteriorly, widest in the middle, tapering and pointed apically, few short setae posteriorly visible from lateral view; inner surface with five or six peg-like setae in basal half. Measurements and ratios as in table 2A-C.

Fifth instar larva: unknown.

Host plants. Unknown.

Distribution. Nigeria.

Material examined. Holotype $\widehat{o}$, Nigeria: Ife, 29 December 1970 (J. T. Medler) (BMNH). Dry mounted. 
PARATYPeS. Nigeria: $1 \hat{\jmath}, 1$, same data as holotype but 10 July 1969 (BMNH).

Comments. Similar to $P$. boxi sp. n. from which it differs in the shape of the genitalia.

\section{Paurocephala minuta Crawford}

Paurocephala minuta Crawford, 1919: 150. Holotype $q$, examined.

Description. Species of the brevicephala type.

Adult: described by Crawford, 1919.

Fifth instar larva: unknown.

Host plants. Unknown.

Distribution. Philippines (Luzon).

Material examined. Holotype + , Philippines, Luzon, Los Baños (P. I. Baker) (USNM). Dry mounted.

Comments. The female holotype is extensively damaged. Based on the presence of the ventrally serrated lateral valvulae it is a species of the brevicephala type.

Paurocephala muiri sp. $\mathrm{n}$.

(figures 17D, 18D, 19D, H, M, 20D)

Description. Species of the psylloptera type.

Adult: similar to $P$. psylloptera differing in the darker overall body coloration and Rs vein with 23-28 setae. Measurements and ratios as in table 2A-C.

Fifth instar larva: unknown.

Host plants. The material examined was collected on Ficus sp. (Moraceae) which could be the host plant.

Distribution. Indonesia (Tanimbar Island).

Material examined. HolotyPE $\widehat{\jmath}$, Indonesia: Tanimbar Island, Larat, December 1907 (F. Muir) (USNM). Dry mounted.

PARATYPES. Indonesia: $1 \widehat{\jmath}, 5 \circ$, same data as holotype (BMNH, MHNG, USNM). Dry and slide mounted.

Comments. P. muiri sp. n. is similar to P. psylloptera Crawford. The best character to separate the two species is the shorter setae associated with antennal rhinaria 8 and 9. The record of $P$. psylloptera by Crawford (1919: 149, Tenimbar Island, Larat) concerns this species.

\section{Paurocephala muta sp. n.}

(figures $22 \mathrm{G}, 24 \mathrm{C}, \mathrm{N}, 25 \mathrm{~K}, 27 \mathrm{E}, 28 \mathrm{~L}$ )

Description. Species of the psylloptera type.

Adult: head and clypeus brown; vertex covered by short setae. Antenna yellow, apical half of segment 4, entire segments 6 and 8-10 brown; segment 9 without a long basal seta. Thorax brown and covered by short setae dorsally, light brown to white laterally and ventrally. Metascutellar horn small, pointed apically. Forewing transparent to white; veins yellow to brown; pterostigma white. Forewing with short setae on veins (vein Rs with 18-23 setae); Rs vein marginally curved in apical third. Surface spinules present in all cells of forewing; radular spinules present in $\mathrm{m}_{2}$, reduced in $\mathrm{cu}_{1}$. Legs white to yellow, last tarsal segment brown. Meracanthus long, curved laterally, pointed apically. Abdomen brown, distal sternites white, genitalia yellow. Male paramere long, almost straight posteriorly, widest basally, rounded 
apically, long setae posteriorly visible from lateral view; inner surface with two hooks apically, six to eight stout setae in basal half. Measurements and ratios as in table $2 \mathrm{~A}-\mathrm{C}$.

Fifth instar larva: unknown.

Host plants. Adults were collected on Ficus sp. (Moraceae) which could be the host and Sterculia sp. (Sterculiaceae).

Distribution. India.

Material examined. HolotyPE ô, India: Keraha, Christian College, 5 April 1968, Ficus sp. (Malabat) (BMNH). Dry mounted.

PARATYPES. India: $13 \hat{\jmath}, 10 \%$, same data as holotype; 11 $\hat{\jmath}, 13+$, Chengannur, 23 May 1991, Sterculia sp. (Prathapan) (BMNH, NHMB). Dry and slide mounted.

Comments. Similar to $P$. oceanica sp. $n$. from which it differs in the absence of surface spinules in cell $\mathrm{c}+\mathrm{sc}$ of the forewing and in details of the paramere.

Paurocephala oceanica sp. $\mathrm{n}$.

(figures 21B, 23B, 24E, 25B, 26B, 28B)

Description. Species of the psylloptera type.

Adult: head orange to yellow, coronal suture and area around median ocellus brown; vertex coverted by long setae. Clypeus yellow. Antenna yellow, apical half of segment 4, entire segments 6 and 8-10 brown; segment 9 without a long basal seta. Thorax orange with yellow markings along body axis and covered by short setae dorsally, orange to light brown laterally and ventrally. Metascutellar horn small, pointed apically. Forewing transparent to white; veins yellow, apically brown to forewing margin; pterostigma yellow to white. Forewing with long setae on veins (vein Rs with 13-18 setae); Rs vein marginally curved in apical third. Surface spinules present in all cells of forewing; radular spinules present in $\mathrm{m}_{1}, \mathrm{~m}_{2}$, reduced in $\mathrm{cu}_{1}$. Legs white to yellow, tarsal segments brown. Meracanthus long, curved laterally, apically pointed. Abdomen including genitalia, yellow, basal tergites brown. Male paramere long, widest at basal third, almost straight posteriorly, pointed apically, long setae posteriorly visible from lateral view; inner surface with two hooks apically, two to four stout setae at base. Measurements and ratios as in table $2 \mathrm{~A}-\mathrm{C}$.

Fifth instar larva: unknown.

Host plants. Adults were collected on Trema tomentosa (Roxb.) H. Hara (Ulmaceae) which could be the host.

Distribution. India (Andaman Islands).

Material examined. HoLOTYPE $\hat{0}$, India: Andaman Islands, Garacherama, 28 December 1988, Trema tomentosa (Bhumannavara) (BMNH). Dry mounted.

PARATYPES. India: $5 \hat{\jmath}, 9$, same data as holotype (BMNH, NHMB). Dry and slide mounted.

Comments. $P$. oceanica sp. $\mathrm{n}$. is similar to $P$. dayak sp. n. from which it is distinguished in the absence of a long basal seta on antennal segment 9 .

Paurocephala palawanensis sp. $\mathrm{n}$.

(figures 21D, 23D, 24G, 25D, 26D, 28D)

Description. Species of the psylloptera type.

Adult: head brown, vertex yellow, genae white; vertex covered by long setae. Clypeus brown. Antenna yellow, apical third of segment 4, entire segment 6 , apical 
two-thirds of segment 8 , and entire segments 9 and 10 brown; segment 9 without a long basal seta. Thorax brown and covered by short setae dorsally, yellow to light brown laterally and ventrally. Metascutellar horn small, pointed apically. Forewing transparent to white; veins yellow, apically light brown to forewing margin; pterostigma white. Forewing with short setae on veins (vein Rs with 24-33 setae); Rs vein marginally curved in apical third. Surface spinules present in all cells of forewing; radular spinules present in $\mathrm{m}_{2}$, reduced in $\mathrm{cu}_{1}$. Legs white to yellow, tarsal segments brown. Meracanthus long, curved laterally, pointed apically. Abdominal tergites brown, sternites white, genitalia yellow. Male paramere long, almost straight posteriorly, widest in the middle, long setae posteriorly visible from lateral view; inner surface with two hooks apically, two to four stout setae basally. Measurements and ratios as in table $2 \mathrm{~A}-\mathrm{C}$.

Fifth instar larva: colour unknown. Antenna 3-segmented, flagellum indistinctly subdivided; segments 1 without a sectaseta, segment 2 with one sectaseta, flagellum with five sectasetae grouped in 2, 2 and 1. Dorsal sclerites covered by sectasetae as large as marginal ones and inconspicuous to short simple setae. Forewing pad with 9 or 10 sectasetae and one or two inconspicuous simple setae marginally; humeral lobe lacking. Caudal plate rounded marginally, apically shallow excavated concavely; sectasetae present dorsally and marginally. Arolium triangular and petiolate, expanded basally. Anus ventral, outer circumanal ring with a single row of pores. Measurements and ratios as in table 3.

Host plants. Trema orientalis (L.) Blume.

Distribution. Philippines (Palawan).

Material examined. HolotyPe ô, Philippines: Palawan, Mt St Paul N. P., SabangCabayugang road, 25 January 1988, Trema orientalis (J. H. Martin) (BMNH). Dry mounted.

PARATyPes. Philippines: $1 \hat{\jmath}, 9 q$ and 4 larvae, same data as holotype (BMNH, NHMB). Dry and slide mounted.

Comments. $P$. palawanensis sp. $\mathrm{n}$. is similar to $P$. setifera (Crawford) from which it is distinguished by the shorter setiferation on body and forewing veins.

\section{Paurocephala papuana sp. $\mathrm{n}$.}

(figures $17 \mathrm{E}, 18 \mathrm{E}, 19 \mathrm{E}, \mathrm{N}, 20 \mathrm{E}$ )

Description. Species of the psylloptera type.

Adult: head dark brown; vertex covered by short setae. Clypeus brown. Antenna brown; segment 9 with a long seta in basal third. Thorax brown with yellow to orange markings along body axis and covered by inconspicuous setae dorsally, orange to brown laterally and ventrally. Metascutellar horn small, pointed apically. Forewing transparent; veins light brown; pterostigma white. Forewing with inconspicuous setae on veins (vein Rs with 11-15 setae); Rs vein marginally curved towards distal third. Surface spinules absent or confined to cells $\mathrm{cu}_{1}$ and apex of $\mathrm{m}_{1}$ and $\mathrm{m}_{2}$; radular spinules present in $\mathrm{m}_{2}$, reduced in $\mathrm{cu}_{1}$. Legs brown, tarsal segments darker. Meracanthus long, straight laterally, pointed apically. Abdomen including genitalia, brown. Male paramere long, widest basally, apical third marginally curved posteriorly, few relatively short setae, posteriorly visible from lateral view; inner surface with two hooks apically, one to three stout setae basally. Measurements and ratios as in table $2 \mathrm{~A}-\mathrm{C}$.

Fifth instar larva: unknown. 
Host plants. Unknown, some adults were collected by fogging (?) Piper sp. (Piperaceae) and Piptrus sp. (Urticaceae).

Distribution. Papua New Guinea.

Material examined. Holotype ${ }^{\wedge}$, Papua New Guinea: Morobe, Mt Kaindi, 1150 2300 m, 9-19 November 1992 (Y. Basset) (NHMB). Dry mounted.

Paratypes. Papua New Guinea: $2 \hat{\jmath}, 3+$, same data as holotype (MHNG). Dry and slide mounted.

Comments. P. papuana sp. $\mathrm{n}$. is similar to $P$. psylloptera Crawford from which it is distinguished by the darker body coloration and the apical third of the male paramere which is directed posteriorly.

\section{Paurocephala phalaki Mathur}

Paurocephala phalaki Mathur, 1975: 58. Holotype ${ }^{\star}$, India: Bengal, Tista village, 27 October 1965, (V. R. Phalak) (IFRI), not examined.

Description. Species of the chonchaiensis type.

Adult: described by Mathur (1975).

Fifth instar larva: described by Mathur (1975).

Host plants. According to Mathur (1975) the common name of the host plant in Bengal is 'khasare'. No information was found with this local plant name, but a similar name 'khesari' refers to Lathyrus sativus L. (Fabaceae) (E. Gauda, personal communication).

Distribution. India (Bengal) (Mathur, 1975), (Madras) (Kandasamy, 1986).

Comments. The description provided by Mathur (1975) agrees with P. bifasciata Kuwayama differing only in body setiferation. Only two long setae on genae were mentioned by Mathur (1975) for $P$. phalaki Mathur, whereas the head of $P$. bifasciata Kuwayama is completely covered by long setae. No material of the former was available for examination.

\section{Paurocephala polaszeki sp. $\mathrm{n}$.}

(figures $22 \mathrm{~F}, 24 \mathrm{~B}, \mathrm{M}, 25 \mathrm{~J}, 27 \mathrm{D}, 28 \mathrm{~K}, \mathrm{~N}$ )

Description. Species of the psylloptera type.

Adult: material at hand is teneral and unless otherwise stated overall body colour is white to yellow. Vertex covered by short setae. Antenna white, apical half of segments 4, 6 and 8 and entire segment 9 and 10 brown; segment 9 without a long basal seta. Thorax covered by inconspicuous setae dorsally. Metascutellar horn small, pointed apically. Forewing whitish; veins light yellow, apically brown to forewing margin. Forewing with long setae on veins (vein Rs with 13-22 setae); Rs vein curved in apical half. Surface spinules present in all cells of forewing; radular spinules present in $\mathrm{m}_{2}$, reduced in $\mathrm{cu}_{1}$. Legs white, tarsal segments brown. Meracanthus long, curved laterally and pointed apically. Male paramere long, widest in the middle, rounded apically, long setae posteriorly visible from lateral view; inner surface with two hooks apically, one to three stout setae basally. Measurements and ratios as in table $2 \mathrm{~A}-\mathrm{C}$.

Fifth instar larva: antenna yellow to light brown, apical third of flagellum brown; 3-segmented, flagellum indistinctly subdivided; segment 1 without a sectaseta, segment 2 with one sectaseta, flagellum with five sectasetae grouped in 2,2 and 1. Dorsal sclerites brown, covered by sectasetae as large as marginal ones with few inconspicuous simple setae. Wing pads light brown, basally darker; forewing pad 
with seven to nine sectasetae and one to three inconspicuous simple setae marginally; humeral lobe lacking. Caudal plate brown, with shallow tubercle-like extentions marginally, apically strongly excavated concavely; sectasetae present dorsally and marginally with few inconspicuous setae. Arolium triangular and petiolate, expanded basally. Anus ventral, outer circumanal ring with a single row of pores. Measurements and ratios as in table 3.

Host plants. Trema orientalis (L.) Blume (Ulmaceae).

Distribution. Bangladesh.

Material examined. HolotyPE ô, Bangladesh: Koitta Manikgonj, 25 March 2000, Trema orientalis (Z. Islam) (BMNH). Dry mounted.

PARATYPES. Bangladesh: $3 \hat{\jmath}, 4 q$ and 4 larvae, same data as holotype; $1 \hat{\jmath}$ and 2 larvae same data but 15 February 2000 (A. Polaszek) (BMNH, NHMB).

Comments. Similar to $P$. psylloptera from which it differs in the absence of a basal seta on antennal segment 9 .

\section{Paurocephala psylloptera Crawford}

(figures 17C, 18C, H, 19C, G, L, 20C, 41)

Paurocephala psylloptera Crawford, 1914: 294; Lectotype $\hat{\sigma}$ (dry mounted) (USNM), examined, by present designation.

Description. Species of the psylloptera type.

Adult: head and clypeus brown; vertex covered by short setae. Antenna brown, segments 1-3 yellow; segment 9 with a long seta in basal quarter. Thorax brown and covered by short setae dorsally, yellow to brown laterally and ventrally. Metascutellar horn small, pointed apically. Forewing transparent; veins light brown; pterostigma yellow to brown. Forewing with inconspicuous setae on veins (vein Rs with 12-18 setae); Rs vein marginally curved in distal third. Surface spinules absent or confined to cell $\mathrm{cu}_{1}$ and apex of $\mathrm{m}_{1}$ and $\mathrm{m}_{2}$; radular spinules present in $\mathrm{m}_{2}$, reduced in $\mathrm{cu}_{1}$. Legs yellow to brown, tarsal segments darker. Meracanthus long, straight laterally, pointed apically. Abdomen including genitalia, yellow to light brown. Male paramere long and straight, truncate apically, few short setae posteriorly visible from lateral view; inner surface with two hooks apically, a row of stout setae confined in basal third. Measurements and ratios as in table $2 \mathrm{~A}-\mathrm{C}$.

Fifth instar larva: unless otherwise stated overall body coloration yellow to white. Apical third of flagellum brown; antenna 3-segmented, flagellum indistinctly subdivided; segments 1 without a sectaseta, segment 2 with one sectaseta, flagellum with five sectasetae grouped in 2, 2 and 1. Dorsal sclerites covered by sectasetae as large as marginal ones with few inconspicous to short simple setae. Forewing pad with seven to nine sectasetae and three to six inconspicuous simple setae marginally; humeral lobe lacking. Caudal plate with shallow tubercle-like extensions marginally, apical one distinctly excavated concavely; sectasetae present dorsally and marginally with few inconspicuous simple setae. Arolium triangular and petiolate, expanded basally. Anus ventral, outer circumanal ring with a single row of pores. Measurements and ratios as in table 3.

Host plants. Ficus spp. (Braza and Calilung, 1981).

Distribution. Philippines (Crawford, 1914; Miyatake, 1971; Braza and Calilung, 1981; Yang et al., 1986), Indonesia (Sumatra) (Crawford, 1928); (Sulawesi) and Malaysia (Sabah). Other records in the literature are based on misidentifications (see comments). 
Material examined. Philippines: Lectotype $\hat{\sigma}$, paralectotypes $\hat{\jmath}, 2+$, Los Baños $\mathbf{P}$.

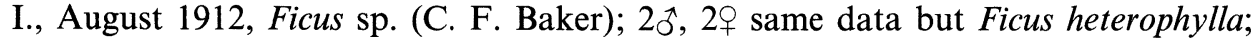
3ิ, 3ㅇ, 4 larvae same data but Laguna, Mt Makiling, 30 December 1976, Ficus minahassae (R. D. Braza); 11 $\widehat{0}, 16$, 11 larvae same data but 3 December 1988, Ficus sp. (A. T. Barrion); 10ð, 21ㅇ, Mindanao, Claveria, Misamis Oriental, 7 July 1988, Ficus sp. (A. T. Barrion); 2ㅇ, Davao experimental station, May to July 1962,

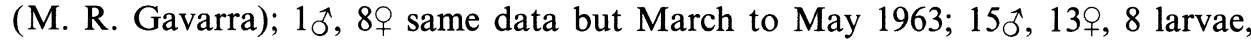
Palawan, Inagauan River valley, 30 January 1988, Ficus sp. ant attended (J. H. Martin); $1 \hat{\jmath}, 2$ s same data but 31 January 1988. Malaysia: $1{ }^{\jmath}$, Sabah, Mt Kinabalu, 1700 m, 22 March 1983 (S. Nagai). Indonesia: 11 $\widehat{\jmath}$, 19, 3 larvae, Sulawesi Utara, Dolodua-Molibagu road, ca $200 \mathrm{~m}, 29$ May 1985, ? Moraceae/Urticaceae (J. H.

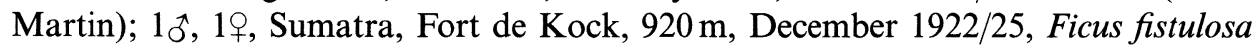
(E. Jacobson) (BMNH, MHNG, NHMB, USNM). Dry and slide mounted and preserved in alcohol.

Comments. Since its original description, the name $P$. psylloptera Crawford has been applied abusively and most records in fact refer to other species. For this reason it it necessary to stabilize the name in designating a lectotype. The two varieties setifera and maculipennis described by Crawford (1914) and Uichanco (1921), respectively, are treated as species in the present work. The records from India (Crawford, 1915, 1924; Mathur, 1975; Ramakrishna Ayyar, 1924), Tenimber Islands (Crawford, 1919) and Fiji (Laing, 1922) are new taxa (P. muta sp. n., $P$. muiri sp. n. and $P$. calcarata sp. n., respectively). The records from Taiwan (Boselli, 1930; Kuwayama, 1931; Yang, 1984) refer to P. sauteri (Enderlein) and (Takahashi, 1936), P. trematos Yang et al. while the records from Japan (Kuwayama, 1943; Miyatake, 1964, 1965; Sasaki, 1954) refer to P. trematos Yang et al.

Paurocephala pterospermi sp. $\mathrm{n}$.

(figures 32B, D, J, 33F, L, 34F, 46)

Description. Species of the brevicephala type.

Adult: head yellow, sides along coronal suture and genae white; vertex covered by inconspicuous setae. Clypeus light brown. Antenna yellow, segment 8 orange to brown; segment 7 with a long basal seta. Thorax yellow to orange with white markings along body axis and covered by inconspicuous setae dorsally, yellow laterally and ventrally. Metascutellar horn small, wide basally, blunt apically. Forewing transparent to light yellow; veins yellow, apically brown to forewing margin, central part of vein A brown; pterostigma white, brown apically. Forewing with short setae on veins (vein Rs with 19-21 setae); Rs vein marginally curved throughout. Surface spinules present in all cells of forewing, reduced in c + sc; radular spinules lacking. Legs including tarsal segments, white, femur yellow. Meracanthus short, almost straight laterally, rounded apically. Abdominal tergites yellow to orange, sternites brown, genitalia yellow. Male paramere elongate, widest basally, tapering apically and directed posteriorly in apical half, few long setae posteriorly visible from lateral view; inner surface with a hook apically. Measurements and ratios as in table $2 \mathrm{~A}-\mathrm{C}$.

Fifth instar larva: colour unknown. Similar to $P$. brevicephala but with anus in terminal position. Measurements and ratios as in table 3.

Host plants. Pterospermum sp. (Sterculiaceae).

Distribution. Philippines (Palawan) and Malaysia (Sabah). 

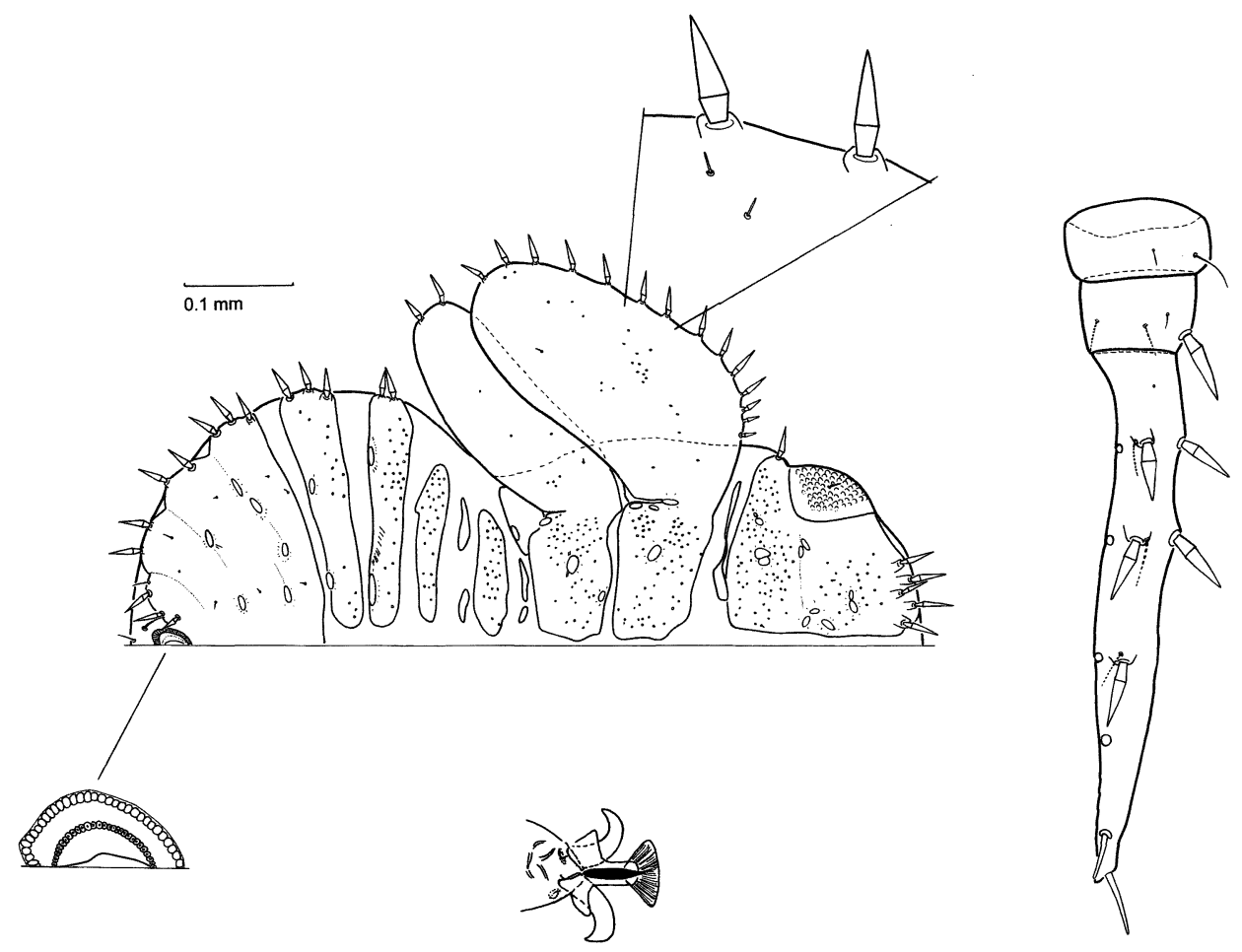

FIG. 46. Fifth instar larva: Paurocephala pterospermi.

Material examined. Holotype ô, Philippines: Palawan, Inagauan River, $700 \mathrm{~m}$, 1 February 1988, Pterospermum sp. (J. H. Martin) (BMNH). Dry mounted.

PARATYPES. Philippines: $8 \hat{\jmath}, 11$, 43 larvae, same data as holotype but 30 January to 1 February 1988. Malaysia: $2 \hat{\jmath}, 7$, Sabah, Poring Hot Springs, $500 \mathrm{~m}, 7$ May 1987 (D. Burckhardt and I. Löbl); 1ㅇ, same data but Mt Kinabalu, 600 m, 2 May 1987, Leptospermum sp. (MHNG, NHMB). Dry and slide mounted.

Comments. $P$. pterospermi sp. $\mathrm{n}$. is similar to $P$. ambigua sp. $\mathrm{n}$. from which it is distinguished by the paramere shape and long setae on forewing veins. The material collected in Sabah differs in having shorter setae on forewing veins and reduced areas of surface spinules in forewing cells, which is considered here as variation.

Paurocephala robusta sp. $\mathrm{n}$.

(figures $31 \mathrm{C}, 32 \mathrm{~F}, \mathrm{~L}, 33 \mathrm{C}, \mathrm{I}, 34 \mathrm{C}, 45$ )

Description. Species of the brevicephala type.

Adult: head brown, darker towards lateral ocelli; vertex covered by inconspicuous setae. Clypeus light brown. Antenna yellow to light brown; segment 7 with a long basal seta. Thorax entirely brown and covered by inconspicuous setae dorsally. Metascutellar horn small, basally wide, blunt apically. Forewing light brown, veins yellow, apically brown to forewing margin, central part of vein A brown; pterostigma white. Forewing with short setae on veins (vein Rs with 16-20 setae); Rs vein marginally curved throughout. Surface spinules present in all cells of forewing; radular spinules lacking. Legs including tarsal segments, yellow to white, femur brown. Meracanthus short, almost straight laterally, rounded apically. Abdomen, 
including genitalia, brown. Male paramere elongate and slender, widest in basal half, tapering apically and marginally curved backwards, few relatively long setae posteriorly visible from lateral view. Measurements and ratios as in table $2 \mathrm{~A}-\mathrm{C}$.

Fifth instar larva: colour unknown. As in P. brevicephala. Measurements and ratios as in table 3 .

Host plants. Unknown.

Distribution. Philippines (Palawan).

Material examined. Holotype ô, Philippines: Palawan, Mt St Paul N. P., SabangCabayugan road, 25 January 1988, ? Convolvulaceae (J. H. Martin) (BMNH). Dry mounted.

Paratypes. Philippines: $6 \hat{0}, 249,11$ larvae, same data as holotype (BMNH, NHMB). Dry and slide mounted.

Comments. Similar to $P$. brevicephala (Crawford) from which it differs in the adult in the absence of a long basal seta on antennal segment 8 . No characters were found to separate the larvae.

\section{Paurocephala russellae Mathur}

Paurocephala russellae Mathur, 1975: 64. Holotype ${ }^{\star}$, India: Dehra Dun, New Forest, 17 June 1932, Kydia calycina (R. N. Mathur) (IFRI), not examined.

Description. Species of the kleinhofiae type.

Adult: described by Mathur (1975).

Fifth instar larva: described by Mathur (1975).

Host plants. Kydia calycina Roxb. (Malvaceae).

Distribution. India (Uttar Pradesh).

Material examined. None.

Comments. No material was available for study. $P$. russellae Mathur is similar to P. kleinhofiae Uichanco from which it is distinguished in its 10-segmented antennae.

\section{Paurocephala sauteri (Enderlein)}

(figures 22B, 23G, 24J, 25G, 26G, 28G)

Agonoscena sauteri Enderlein, 1914: 234. Lectotype + , examined, by present designation.

Paurocephala sauteri (Enderlein); Crawford, 1917: 34.

Paurocephala (Agonoscena) sauteri Enderlein; Kuwayama, 1922: 368.

Paurocephala debregeasiae Yang and Li, 1987, holotype $\widehat{0}$, examined. Syn. n.

Description. Species of the psylloptera type.

Adult: head brown, yellow towards lateral and median ocelli; vertex covered by short setae. Clypeus brown. Antenna brown, segments 1-3 yellow; segment 9 with a long seta in basal quarter. Thorax entirely brown with yellow markings along body axis and covered by inconspicuous setae dorsally. Metascutellar horn large, pointed apically. Forewing including pterostigma transparent; veins brown. Forewing with inconspicuous setae on veins (vein Rs with 10-15 setae); Rs vein marginally curved in apical third. Surface spinules absent or confined to cells in apical half of forewing; radular spinules present in $\mathrm{m}_{2}$, reduced in $\mathrm{cu}_{1}$. Legs yellow to brown, tarsal segments darker. Meracanthus long, laterally curved, pointed apically. Abdominal tergites yellow to light brown, sternites white to light brown, genitalia brown. Male paramere long and almost straight posteriorly, widest in the middle, pointed apically, few short setae posteriorly visible from lateral view; inner surface 
with two hooks apically, one to three stout setae basally. Measurements and ratios as in table $2 \mathrm{~A}-\mathrm{C}$.

Fifth instar larva: no material was available for study. The description of $P$. psylloptera by Yang (1984) concerns $P$. sauteri.

Host plants. Morus alba L. (Moraceae). The records from Celtis tetrandra sinensis (Pers.) Y. C. Tang (Ulmaceae) and from Debregeasia longifolia (Burm. f.) Wedd. (Urticaceae) cannot be confirmed since no larvae were reported.

Distribution. China (Yang and Li, 1987), Taiwan (Enderlein, 1914; Yang et al., 1986) and Thailand.

Material examined. Taiwan: Lectotype + , July 1912 (H. Sauter); 4^, 5 $\odot$, Nantou, Chushan, 19 June 1978, Morus alba (C. T. Yang). China: Holotype (of P. debregeasiae) $\widehat{\sigma}$ and $5 \propto$ paratypes, Yunnan, Xishuang-banna, Jinghong, 4 April 1981, Debregeasia longifolia. Thailand: 4ðิ, 5, Bangkok, 1962, Morus sp. (B. Meksongsee) (BAUC, DEIC, NCHU).

Comments. Yang et al. (1986) demonstrated that $P$. sauteri is morphologically, ethologically and biologically distinct from $P$. psylloptera Crawford. To fix the name sauteri a lectotype is selected here. No larval material was available for examination.

Paurocephala setifera (Crawford), stat. n.

(figures 22E, 24A, L, 25I, 27C, 28J, 44)

Pauropsylla triozoptera setifera Crawford, 1914: 297. Lectotype $\widehat{ }{ }^{\lambda}$, examined, here designated. Paurocephala triozoptera setifera (Crawford); Crawford, 1919: 231

Description. Species of the psylloptera type.

Adult: head yellow, coronal suture and area around median ocellus brown; vertex covered by long setae. Clypeus brown. Antenna yellow, segment 8 and 9 brown; segment 9 without a long basal seta. Thorax brown and covered by long setae dorsally, yellow to light brown laterally and ventrally. Metascutellar horn small, pointed apically. Forewing transparent to light yellow, veins yellow to light brown, apically light brown to forewing margin; pterostigma light yellow. Forewing with long setae on veins (vein Rs with 28-35 setae); Rs vein marginally curved in apical third. Surface spinules present in all cells of forewing; radular spinules present in $\mathrm{m}_{2}$ and $\mathrm{cu}_{1}$. Legs yellow, last tarsal segment dark brown. Meracanthus long, curved laterally, pointed apically. Abdominal tergites brown, sternites including genitalia, yellow. Male paramere long, widest basally, tapering towards apex, long setae posteriorly visible from lateral view; inner surface with two hooks apically, two to five stout setae in basal third. Measurements and ratios as in table $2 \mathrm{~A}-\mathrm{C}$.

Fifth instar larva: colour unknown. Similar to $P$. palawanensis and $P$. boehmeriae. Measurements and ratios as in table 3.

Host plants. Trema orientalis (L.) Blume (Ulmaceae).

Distribution. Philippines (Luzon) (Crawford, 1914) and Mindanao.

Material examined. Philippines: Lectotype $\widehat{\jmath}$, paralectotypes $2 \hat{\jmath}, 1 \%$, Luzon, Los Baños, 1927 (C. F. Baker); 4ð̊, 5ㅇ, Camarines, 28 December 1974, Trema orientalis

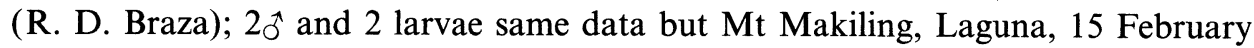
1976; 3ㅅ, 4ㅇ, Mindanao, February 1962 (M. R. Gavarra) (BMNH, USNM). Dry and slide mounted.

Comments. P. setifera (Crawford) is distinguished from other species of the psylloptera type by the longer and larger number of seta on forewing veins 
(figure 22E). Most records citing $P$. setifera (Crawford) need confirmation. To fix the identity and status of $P$. setifera a lectotype is designated here.

\section{Paurocephala similis sp. $\mathrm{n}$.}

(figures 13A, 14A, 15A, G, 16A)

Description. Species of the wilderi type.

Adult: head brown; vertex covered by inconspicuous setae. Antenna brown, segments 1-3 and basal half of segment 4 yellow; segment 9 without a long basal seta. Thorax brown with light yellow markings along body axis and covered by short setae dorsally, brown laterally and ventrally. Metascutellar horn small, blunt apically. Forewing transparent to light yellow; veins light brown; pterostigma white. Forewing with inconspicuous setae on veins (vein Rs with 14-17 setae); Rs vein marginally curved in apical third. Surface spinules absent or confined to cell $\mathrm{cu}_{1}$ and apex of $\mathrm{m}_{1}$ and $\mathrm{m}_{2}$; radular spinules present in $\mathrm{m}_{2}$, reduced in $\mathrm{cu}_{1}$. Legs yellow, tarsal segments brown. Meracanthus long, straight laterally, rounded apically. Abdominal tergites brown, sternites white, genitalia yellow. Male paramere long and S-shaped, widest basally, few short setae posteriorly visible from lateral view; inner surface with a row of four to six stout setae in basal half posteriorly. Measurements and ratios as in table $2 \mathrm{~A}-\mathrm{C}$.

Fifth instar larva: unknown.

Host plants. Unknown.

Distribution. Indonesia (Sulawesi).

Material examined. Holotype $\widehat{\jmath}$, Indonesia: Sulawesi, Sulawesi Utara, DumogaBone N. P., ca 190 m, 9-16 May 1985 (J. S. Noyes) (BMNH). Dry mounted.

PARATYPES. Indonesia: $2 \hat{0}, 12 \circ$, same data as holotype (BMNH, NHMB). Dry and slide mounted.

Comments. P. similis sp. $\mathrm{n}$. is distinguished from the other species of the wilderi type by the paramere which is S-shaped in profile.

\section{Paurocephala sinuata sp. $\mathrm{n}$.}

(figures 6B, G, 7B, E, H, K, 8K)

Description. Species of the gossypii type.

Adult: head and clypeus brown; vertex covered by inconspicuous setae. Antenna yellow, apical half of segments 4 and 6 and entire segments 8-10 brown; segment 9 without a long basal seta. Thorax brown and covered by inconspicuous setae dorsally, yellow to light brown laterally and ventrally. Metascutellar horn small, blunt apically. Forewing transparent to light yellow; veins yellow to light brown; pterostigma yellow. Forewing with inconspicuous setae on veins (vein Rs with 18-22 setae); Rs vein strongly curved in distal third. Surface spinules present in all cells of forewing except in $\mathrm{c}+\mathrm{sc}$; radular spinules lacking. Legs including tarsal segments, yellow. Meracanthus short, almost straight laterally, rounded apically. Abdomen, including genitalia, yellow to light brown. Male paramere short, widest in basal third, rounded apically, few short setae posteriorly visible from lateral view; inner surface with five or six peg-like setae situated between basal fifth and distal third. Measurements and ratios as in table $2 \mathrm{~A}-\mathrm{C}$.

Fifth instar larva: unknown.

Host plants. A series of adults were collected on Cnestis ferruginea Vahl ex DC. (Connaraceae). 
Distribution. Ghana and Nigeria.

Material examined. HolotyPE ô, Ghana: Volta Region, Kpeve, March 1943, Cnestis ferruginea (H. E. Box) (BMNH). Dry mounted.

PARATYPES. Ghana: $2 \hat{\jmath}, 2 \circ$, same data as holotype. Nigeria: $1 \hat{\jmath}$, Ife, 30 June 1970 (J. T. Medler); 1 $\hat{\jmath}$, same data but 10 July 1969; 1ㅇ, same data but 29 December 1970; 39, same data but 30 June 1970; 10, same data but 10 February 1973; 3ㅇ, same data but Umuahia, 10 April 1975; 2ิึ, same data but 8 and 11 April 1973 (BMNH, NHMB). Dry and slide mounted.

Comments. $P$. sinuata sp. $\mathrm{n}$. is close to $P$. hollisi sp. n. but differs in the larger body dimensions and the number of peg-like setae on the inner face of the paramere.

\section{Paurocephala stephaniella sp. $\mathrm{n}$. \\ (figures 29C, F, I, 30C, F)}

Description. Species of the brevicephala type.

Adult: head and clypeus light brown; vertex covered by inconspicuous setae. Antenna white, segment 8 brown; segment 7 or 8 without a long basal seta. Thorax white and covered by inconspicuous setae dorsally, light brown laterally and ventrally. Metascutellar horn large, basally wide, pointed apically. Forewing transparent, with a brown pattern apically and two smaller lighter ones towards centre of forewing and in cell $\mathrm{cu}_{2}$; veins white to yellow, apically light brown to forewing margin; pterostigma white, brown basally and apically. Forewing with inconspicuous setae on veins (vein Rs with 11-12 setae); Rs vein curved in basal third and apically, forming an S-shape. Surface spinules sparse in cells of forewing, absent in c $+\mathrm{sc}$, and confined apically in $r_{1}$ and $c_{1}$; radular spinules lacking. Legs white, tarsal segments brown. Meracanthus short, straight laterally, rounded apically. Abdomen, including genitalia, white; female unknown. Male paramere elongate, widest basally and directed posteriorly, few relatively long setae posteriorly visible from lateral view. Measurements and ratios as in table $2 \mathrm{~A}-\mathrm{C}$.

Fifth instar larva: unknown.

Host plants. Unknown.

Distribution. Indonesia (Sulawesi).

Material examined. HolOtyPe ô and PARATYPE $\hat{\sigma}$, Indonesia: Sulawesi, Sulawesi Utara, Dumoga-Bone N. P., 400 m, 11 February 1985 (R. Ent. Soc. Lond., Project Wallace) (BMNH). Dry and slide mounted.

Comments. P. stephaniella sp. $\mathrm{n}$. is distinguished from the other species of the brevicephala type by the brown band on forewing.

\section{Paurocephala stigmaticalis $\mathrm{sp} . \mathrm{n}$.}

(figures 22D, 27B, 28I)

\section{Description. Species of the psylloptera type.}

Adult: head white, coronal suture and area around median ocellus brown; lateral and median ocelli connected by a thin brown line; vertex covered by short setae. Clypeus brown. Antenna yellow, segments 4, 6-10 light brown; segment 9 without a long basal seta. Thorax white and covered by inconspicuous setae dorsally, yellow to light brown laterally and ventrally. Metascutellar horn small, blunt apically. Forewing transparent to white; veins yellow to brow; pterostigma brown. Forewing with short inconspicuous setae on veins (vein Rs with 14-16 setae); Rs vein marginally curved in the middle. Surface spinules mainly confined to cells in apical half of 
forewing and in cell $\mathrm{cu}_{2}$; radular spinules lacking. Legs white, tibia and tarsal segments brown. Meracanthus long, laterally curved, pointed apically. Abdomen white, basal tergites brown, female genitalia white to yellow. Male unknown. Measurements and ratios as in table $2 \mathrm{~A}-\mathrm{C}$.

Fifth instar larva: unknown.

Host plants. The two specimens at hand were collected on Trema orientalis (L.) Blume (Ulmaceae) which could be the host.

Distribution. Malaysia (Sabah).

Material examined. Holotype $q$ and PARATyPe $q$, Malaysia: Sabah, Crocker Range, Kimanis rd., mile 10, 1150 m, 13 May 1982, Trema orientalis (D. Burckhardt) (MHNG). Dry and slide mounted.

Comments. P. stigmaticalis sp. $\mathrm{n}$. is distinguished from the other species of the psylloptera type by the long thin brown pterostigma which is more than half length of vein Rs.

\section{Paurocephala sulawesiana sp. $\mathrm{n}$. (figures 31D, 32G, M, 33D, J, 34D)}

Description. Species of the brevicephala type.

Adult: head yellow, white towards genae; vertex covered by inconspicuous setae. Clypeus yellow. Antenna yellow to orange, segment 8 brown; segment 7 with a long basal seta. Thorax orange to light brown and covered by inconspicuous setae dorsally, yellow laterally and ventrally. Metascutellar horn small, wide basally, blunt apically. Forewing transparent to light yellow; veins yellow, apically light brown to forewing margin, central part of vein A brown; pterostigma white. Forewing with short setae on veins (vein Rs with 11-14 setae); Rs vein marginally curved in basal half. Surface spinules present in all cells of forewing, reduced in $\mathrm{c}+\mathrm{sc}$; radular spinules lacking. Legs yellow, tarsal segments white. Meracanthus short, almost straight laterally, rounded apically. Abdominal tergites brown, sternites and genitalia white to yellow. Male paramere elongate widest basally and tapering apically, apical half directed posteriorly, few relatively long setae posteriorly visible from lateral view. Measurements and ratios as in table $2 \mathrm{~A}-\mathrm{C}$.

Fifth instar larva: colour unknown. Similar to $P$. brevicephala. Measurements and ratios as in table 3 .

Host plants. The material has been collected on ? Zyzygium sp. (Myrtaceae) which is an unlikely host.

Distribution. Indonesia (Sulawesi).

Material examined. HoLotyPE ô, Indonesia: Sulawesi, Sulawesi Utara, DuloduoMolibagu road, ca 200 m, 29 May 1985, ? Syzygium sp. (J. H. Martin) (BMNH). Dry mounted.

PARATYPES. Indonesia: $32 \hat{\jmath}, 30 q$ and numerous larvae, same data as holotype (BMNH, NHMB). Dry and slide mounted and preserved in alcohol.

Comments. Similar to P. brevicephala (Crawford) from which it differs in the adult in the absence of a long basal seta on antennal segment 8 . No characters were found to separate the larvae.

Paurocephala trematos Yang et al.

(figures 21E, F, 23E, 24H, 25E, 26E, 28E)

Paurocephala trematos Yang, Yang and Chao, 1986. Holotype ô, Taiwan, not examined. 
Paurocephala tremae Yang and Li, 1987. Holotype $\widehat{0}$, China, examined. Syn. $\mathrm{n}$.

Paurocephala guanxiensis Yang and $\mathrm{Li}, 1987$. Holotype ${ }_{0}^{*}$, China, examined. Syn. $\mathrm{n}$.

Description. Species of the psylloptera type.

Adult: head black to dark brown; vertex covered by short setae. Clypeus brown. Antenna brown, segments 1-3 yellow; segment 9 with a long seta in basal quarter. Thorax entirely brown and covered by inconspicuous setae dorsally. Metascutellar horn small, pointed apically. Forewing transparent to white; veins light brown, apically dark brown to forewing margin; pterostigma white. Forewing with inconspicuous to short setae on veins (vein Rs with 18-22 setae); Rs vein marginally curved in apical third. Surface spinules variable, present in all cells of forewing or sparse and absent in $\mathrm{c}+\mathrm{sc}$; radular spinules present in $\mathrm{m}_{2}$, reduced in $\mathrm{cu}_{1}$. Legs yellow to light brown, tarsal segments darker. Meracanthus long, curved laterally, pointed apically. Abdominal tergites brown, sternites including genitalia, yellow to light brown. Male subgenital plate and basal half of female proctiger brown. Male paramere elongate, widest in the middle, apically rounded, few long setae posteriorly visible from lateral view; inner surface with two hooks apically, two to four stout setae in basal third. Measurements and ratios as in table 2A-C.

Fifth instar larva: no material was available for study.

Host plants. Trema orientalis (L.) Blume (Ulmaceae).

Distribution. China (Yang and Li, 1987), Taiwan (Yang et al., 1986), Japan, Thailand and Malaysia.

Material examined. Taiwan: Paratypes (of P. trematos) 3̧, 3, Taichung, Takeng, 1 April 1986, Trema orientalis (M. M. Yang). Japan: 1ðิ, 2ㅇ, Ryu-Kyu riomote, S Hoshidate, $50 \mathrm{~m}, 7$ October 1994 (C. Lienhard). Thailand: 2ิ, 3ㅇ, Lampang, Doi Mae Tho, 21 April 1998 (K. Matsumoto). China: Holotype (of P. tremae) ô, paratypes 2ર, 2o, Fujian, Fuzhou, 21 October 1974, Trema orientalis; Holotype (of P. guanxiensis) $\widehat{\sigma}$ and paratypes $2 \widehat{\jmath}, 2$, , Guingxi, Liu-zhou, 9 June 1982, Trema orientalis; 1ㅇ, Hong Kong, Lantau, SW Peninsula, Shek Pik, 20 October 1990, Trema ? orientalis (J. H. Martin). Malaysia: 6ㅅ, 17, Sarawak, Gunong Mulu N. P., Base Camp Helipad, 17-30 September 1977, Trema sp. (D. Hollis) (BMNH, BAUC, MNHG, NCHU). Dry and slide mounted.

Comments. The material from Thailand and Japan agrees with type material of $P$. trematos Yang et al. The material from Sarawak is teneral and the coloration differs. Also the extent and density of surface spinules on forewing is different (figure 21E, F). Most of the material from China has longer setae on forewing venation. The mentioned characters are variable in this species.

\section{Paurocephala urenae Russell}

(figures 5C, G, 8I, 36B)

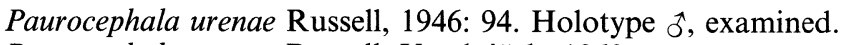
Paurocephala urenae Russell; Vondráček, 1963.

Description. Species of the gossypii type.

Adult: described by Russell (1946). Measurements and ratios as in table 2A-C. Fifth instar larva: described by Russell (1946). Measurements and ratios as in table 3.

Host plants. Urena lobata L. (Malvaceae).

Distribution. Zaire. 
Material examined. Zaire: Holotype $\hat{\jmath}$, paratypes $3 \hat{\jmath}, 4$ 우 and 7 larvae, Gandajika, May 1945, Urena lobata (D. Soyer) (NHMB, USNM). Dry and slide mounted.

Comments. Close to P. abutili sp. n. from which it is distinguished by the overall body coloration which is less dark and shape of the antennae. The fifth instar larvae are difficult to separate. In $P$. abutili sp. n. the sectasetae on body are more slender than in $P$. urenae Russell. The reference of $P$. urenae Russell by Vondráček (1963: 277 ) refers to an undescribed species (see comments under unidentified material of Paurocephala sp. Zaire \#1).

Paurocephala wilderi Crawford

(figures $13 \mathrm{~F}, 14 \mathrm{~F}, 15 \mathrm{~F}, \mathrm{~L}, 16 \mathrm{~F}$ )

Paurocephala wilderi Crawford, 1927: 29. Lectotype $\hat{o}$ (BMNH), examined, by present designation.

Description. Species of the wilderi type.

Adult: head brown; vertex covered by short setae. Clypeus yellow. Antenna brown, segments 1-3 and basal half of segment 4 yellow; segment 9 without a long basal seta. Thorax entirely dark brown and covered by inconspicuous setae dorsally. Metascutellar horn small, blunt apically. Forewing transparent with a brown pattern apically, throughout cell $\mathrm{m}_{1}$ and in adjacent areas of $\mathrm{r}_{2}$ and $\mathrm{m}_{2}$; veins yellow to light brown; pterostigma yellow. Forewing with inconspicuous setae on veins (vein Rs with 13-16 setae); Rs vein marginally curved throughout entire length. Surface spinules present in all cells of forewing except in cell $\mathrm{c}+\mathrm{sc}$; radular spinules present in $\mathrm{m}_{2}$, reduced in $\mathrm{cu}_{1}$. Legs yellow to brown, tarsal segments darker. Meracanthus long, straight laterally, rounded apically. Abdomen including genitalia, brown. Male paramere long, widest basally and tapering apically, apical fifth marginally curved anteriorly, few short setae posteriorly visible from lateral view; inner surface with two hooks apically, group of setae confined anteriorly in basal third. Measurements and ratios as in table $2 \mathrm{~A}-\mathrm{C}$.

Fifth instar larva: unknown.

Host plants. Adults were collected on Ficus sp. (Moraceae) which may be the host.

Distribution. American Samoa (Crawford, 1927).

Material examined. American Samoa: $1 \hat{\jmath}$, lectotype and $2 \hat{\jmath}, 3 q$, paralectotypes, Tutuila, Pago Pago, 22 September 1922, Ficus sp. (O. H. Swezey and G. P. Wilder); $1 \hat{\jmath}, 1$, , same data but 4 August 1940 (O. H. Swezey) (BMNH, MHNG, NHMB, USNM). Dry and slide mounted.

Comments. $P$. wilderi Crawford is distinguished from the other species of the wilderi type by the reduced number of spurs along metatibia and the long pterostigma. To avoid future taxonomic confusion a lectotype is designated here for P. wilderi Crawford.

\section{Unidentified material}

The following material could not be identified due to insufficient material.

Paurocephala sp. Zaire \#1

Paurocephala urenae Russell; Vondráček, 1963: 277, misidentification.

Material examined. 3今̂, 2ㅇ, Yangambi, December 1925, Triumfetta sp. (J. Ghesquiére) (MRAC). Dry and slide mounted.

Comments. The above-mentioned material was cited as $P$. urenae Russell by 
Vondráček (1963). Species of the gossypii type. The antennae are slender as in $P$. urenae Russell but antennal segment ratios are different. The Rs vein of the forewing is more strongly curved than in P. urenae Russell. Adults were collected on Triumfetta sp. (Tiliaceae) which may be the host plant. In the absence of additional material, the species is not formally described.

\section{Paurocephala sp. Nigeria \#1}

Material examined. 2ิึ, 2o, Nigeria, Ibadan, December 1959 and April/June 1960 (F. A. Squire) (BMNH). Slide mounted.

Comments. Species of the gossypii type. Similar to P. boxi sp. n. but differs in the shorter antennae and smaller body dimensions. In the absence of additional material, the species is not formally described.

\section{Paurocephala sp. Hong Kong \#1}

Material examined. 5̧, $4 \uparrow$ and 6 larvae, Hong Kong, Po Shan road, 23 February 1976, Trema orientalis (D. S. Hill) (BMNH). Slide mounted.

Comments. Species of the psylloptera type. Similar to P. boehmeriae sp. n. differing in the larger body dimensions and details of the forewing venation.

\section{Paurocephala sp. Malaysia \#1}

Material examined. Malaysia: 1ㅇ, Perak, Taiping, 300 m, 14 March 1994 (K. Matsumoto) (TULE); 1?, Borneo, Sabah, Tinanamantawaran, near Ranau, $800 \mathrm{~m}$, at light, June 1983 (S. Nagai) (MHNG).

Comments. Species of the psylloptera type. Similar to $P$. distincta sp. n. but differs in the absence of surface spinules on the forewing and the different shape of the genitalia. In the absence of better preserved material, the species is not formally described.

\section{Paurocephala sp. Palawan \#1}

Material examined. Philippines: 5९, Palawan, Inaguana River Valley, 31 January 1988, Ficus sp., ant attended (J. H. Martin) (BMNH). Dry and slide mounted.

Comments. Species of the psylloptera type. Similar to $P$. maculipennis Uichanco and $P$. longiantennata sp. $\mathrm{n}$. from which it differs in the anntennal flagellar ratios and in the dark veins of the forewing. In the absence of additional material including males, the species is not formally described.

\section{Paurocephala sp. Luzon \#1}

Material examined. 2へ, 2q, 2 larvae, Philippines, Luzon, Laguna, 19 October 1976 (R. D. Braza) (BMNH). Slide mounted.

Comments. Species of the psylloptera type. The material is teneral. It differs from other species of the psylloptera type in the reduced spurs on the metatibia (two to four) and the short paramere with six to eight stout setae on inner surface.

\section{Paurocephala sp. Mindanao \#1}

Material examined. 2̧, 1 adult (without abdomen), Philippines, Mindanao, 
Davao (experimental station), December 1961/April 1962/September 1962 (M. R. Gavarra) (BMNH). Slide mounted.

Comments. Species of the brevicephala type. Similar to $P$. robusta $\mathrm{sp} . \mathrm{n}$. in the forewing shape and venation and in the dense surface spinules of the forewing cells. It differs in having prominent stout setae (five or six) on vein $\mathrm{C}+\mathrm{Sc}$ and (one to three) on the anterior margin of pterostigma. In the absence of males and additional better preserved material, the species is not described.

\section{Paurocephala sp. Mindanao \#2}

Material examined. 1 $\hat{\sigma}$, Philippines, Mindanao, Davao (experimental station), August 1962 (M. R. Gavarra); 1ðิ, 19, same data but March 1964 (BMNH). Slide mounted.

Comments. Probably species of the psylloptera type. Due to the bad state of conservation, the lateral processes on first visible abdominal tergite could not been seen. Paramere short without stout setae. The species is characterized by a derived wing form, narrow basally and expanded in distal two-thirds. Brown along forewing veins. It is not described due to lack of well-preserved material.

\section{Paurocephala sp. Mindanao \#3}

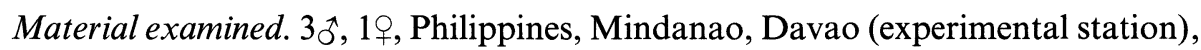
April/May 963 (M. R. Gavarra) (BMNH). Slide mounted.

Comments. Species of the psylloptera type. It is characterized by the elongate forewing and the long setae near antennal rhinaria on segments 8 and 9. Paramere short with six or seven stout setae on inner surface. Not described for the lack of well-preserved material.

\section{Paurocephala sp. Mindanao \#4}

Material examined. 1§ิ, 3ㅇ, Philippines, Mindanao, Davao (experimental station), June 1962/December 1963 (M. R. Gavarra) (BMNH). Slide mounted.

Comments. This species cannot be associated with one of the types. Antenna 8 -segmented with a basal long seta on segment 7 . No bifid setae associated with antennal rhinaria. Male paramere elongate with a row of seven or eight stout setae in basal half of inner face. It differs from the kleinhofiae type in the simple male proctiger and in the lack of dorsal horns on tergites. It differs from the wilderi type in the 8-segmented antenna, which is shorter than head width. Differs from the brevicephala type in the absence of sclerotized, ventrally serrated lateral valvulae. The species is not described for the lack of well-preserved material.

\section{Paurocephala sp. Mindanao \#5}

Material examined. $15 \hat{\jmath}, 19$, Philippines, Mindanao, Davao (experimental station), December 1962 to February 1963 (M. R. Gavarra) (BMNH). Slide mounted.

Comments. Species of the psylloptera type. Similar to $P$. boehmeriae sp. n. and $P$. sp. Hong Kong \#1, but differs in the longer antenna and the forewing venation. 


\section{Paurocephala sp. Sulawesi \#1}

Material examined. Indonesia: 1へ̃, Sulawesi Utara, Dumoga-Bone N. P. $400 \mathrm{~m}$, 19 July 1985, R. Ent. Soc. London. Project Wallace (BMNH).

Comments. Species of the psylloptera type. Similar to $P$. distincta sp. n. but differs in the larger body dimensions and a brown margin on either side of forewing veins. In the absence of additional material including males, the species is not described.

\section{Paurocephala sp. Solomon \#1}

Material examined. 1§ै, 8ㅇ, Solomon Is., Gizo, Gizo, 0-200 m, December 1975 (N. L. H. Krauss) (Bishop Museum via BMNH). Dry mounted.

Comments. Species of the psylloptera type. Similar to P. marginata $\mathrm{sp}$. $\mathrm{n}$. but differs in the smaller body dimensions, the short setae on forewing veins and the light apices of the veins in apical region of forewing.

\section{Paurocephala sp. New Caledonia \#1}

Material examined. 3, New Caledonia, Yahoue, 2 March 1973 (N. L. H. Krauss) (BMNH). Dry mounted.

Comments. Species of the psylloptera type, similar to P. sauteri. More material, including males, is required for proper identification.

\section{Phylogenetic relationships}

The phylogenetic relationships within Paurocephala were examined with cladistic methodology. It would have been desirable to combine adult and larval characters into one analysis, but larval material was available for less than half of the species. The consensus tree of an analysis including all species and using adult and larval characters showed a low resolution. Thus the following two analyses are discussed here.

Analysis 1 includes all 51 Paurocephala spp. with 19 adult characters (table 4). In analysis 2, only those 24 species are included where both adult and larva are known. The adult characters in analysis 2 are the same as those of analysis 1, with nine additional larval characters (table 4).

Analysis 1 resulted, after four iterations, in 120 most parsimonious trees $(\mathrm{L}=$ $184, \mathrm{CI}=68$ and $\mathrm{RI}=95$ ). The (nelsen) consensus tree is shown in figure 47.

Four monophyletic groups resulted. The brevicephala-group (=the ambiguasulawesiana clade) is supported by eight characters of which, the very short head (character 1) and the highly sclerotized female lateral valvulae with ventral serrations (character 19) are autapomorphies. The kleinhofiae-group (=the brendelli-kleinhofiae clade) and the gossypii-group (=the lienhardi-urenae clade) are supported by two characters each. The former is supported by the antenna which is as long as or shorter than head width (character 3 ) and the abdominal tergites which bear a dorsal horn (character 12). The kleinhofiae-group is supported by the metatibia with simple setae laterally (character 11) and the female circumanal ring with two rows of pores (character 18). The Afrotropical species are monophyletic based on the short apical labial segment (character 7). Within the Afrotropical clade $P$. insolita is basal for the absence of pegs on the inner face of the paramere (character 15).

The psylloptera-group (=the curvata-phalaki clade) is supported by the long meracanthus (character 9). P. curvata is basal within the psylloptera-group as it 

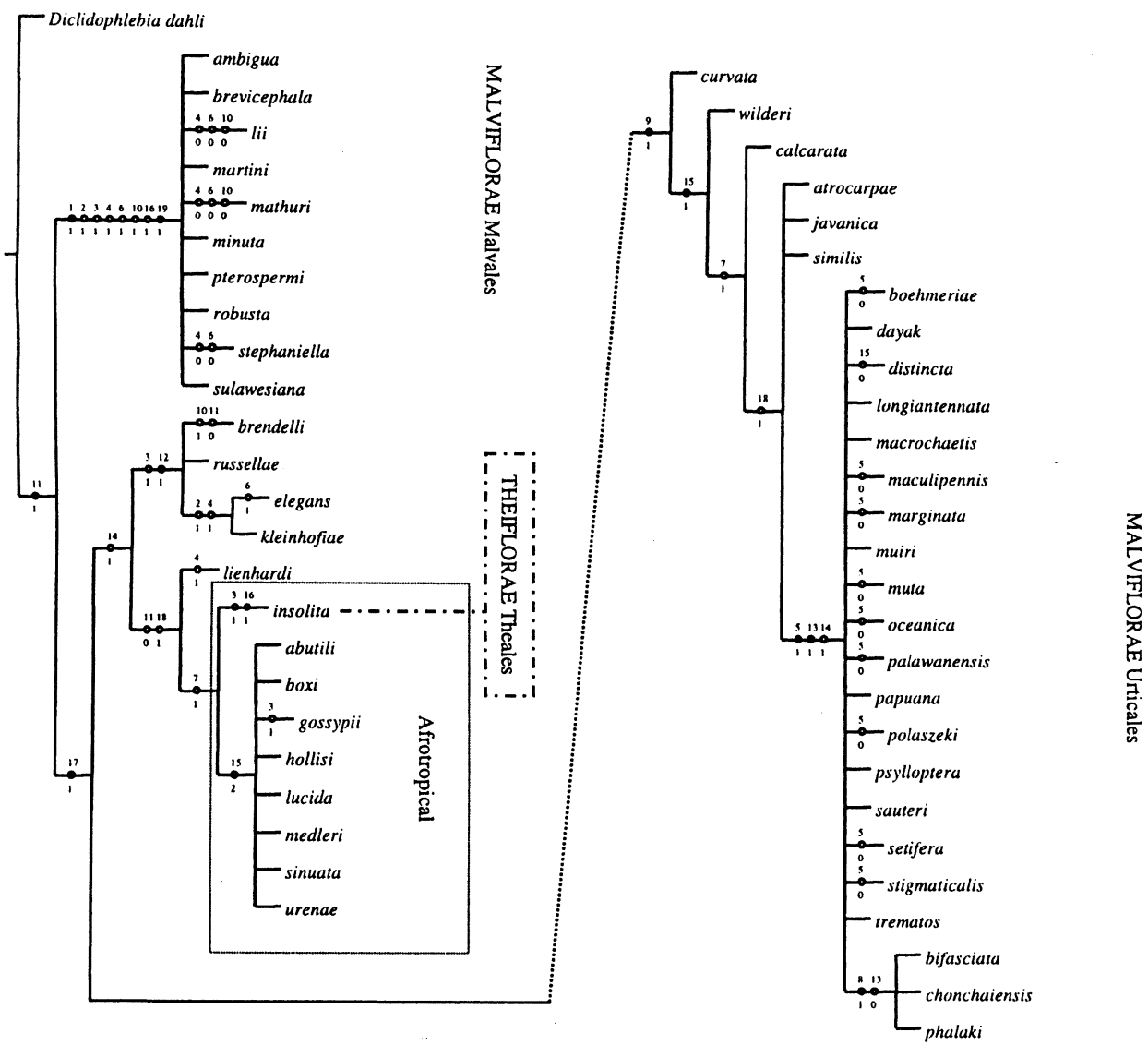

FIG. 47. Cladogram of all Paurocephala species (adult characters) with Diclidophlebia dahli as outgroup.

lacks stout setae on the inner face of the paramere (character 15). The boehmeriaephalaki clade is supported by antennal segment 9 with a long basal seta (character 5), first visible abdominal tergite with lateral patch of long spines on a stalk-like structure (character 13) and male proctiger with distinct posterior sclerites (character 14). Within this clade, the bifasciata-phalaki clade is supported by forewing with distinct brown bands in the middle and apically (character 8 ). The morphology within each of the four monophyletic species groups is relatively homogeneous. It is therefore difficult to find phylogenetically informative characters which would resolve the relationships within the groups.

Analysis 2, after three iterations, resulted in nine most parsimonious trees $(\mathrm{L}=$ $269, \mathrm{CI}=76$ and $\mathrm{RI}=93$ ). The (nelsen) consensus tree is shown in figure 48.

The same four monophyletic species groups appear as in analysis 1 . There is a good support for the brevicephala-group with three additional larval autapomorphies: the circular body form (character 20), the thoracic dorsal sclerites fused to forewing pads (character 24) and presence of humeral lobe (character 25). As in analysis 1 the brevicephala-group is basal to the other three groups. There are no larval characters supporting the latter clade as well as the kleinhofiae- and the 


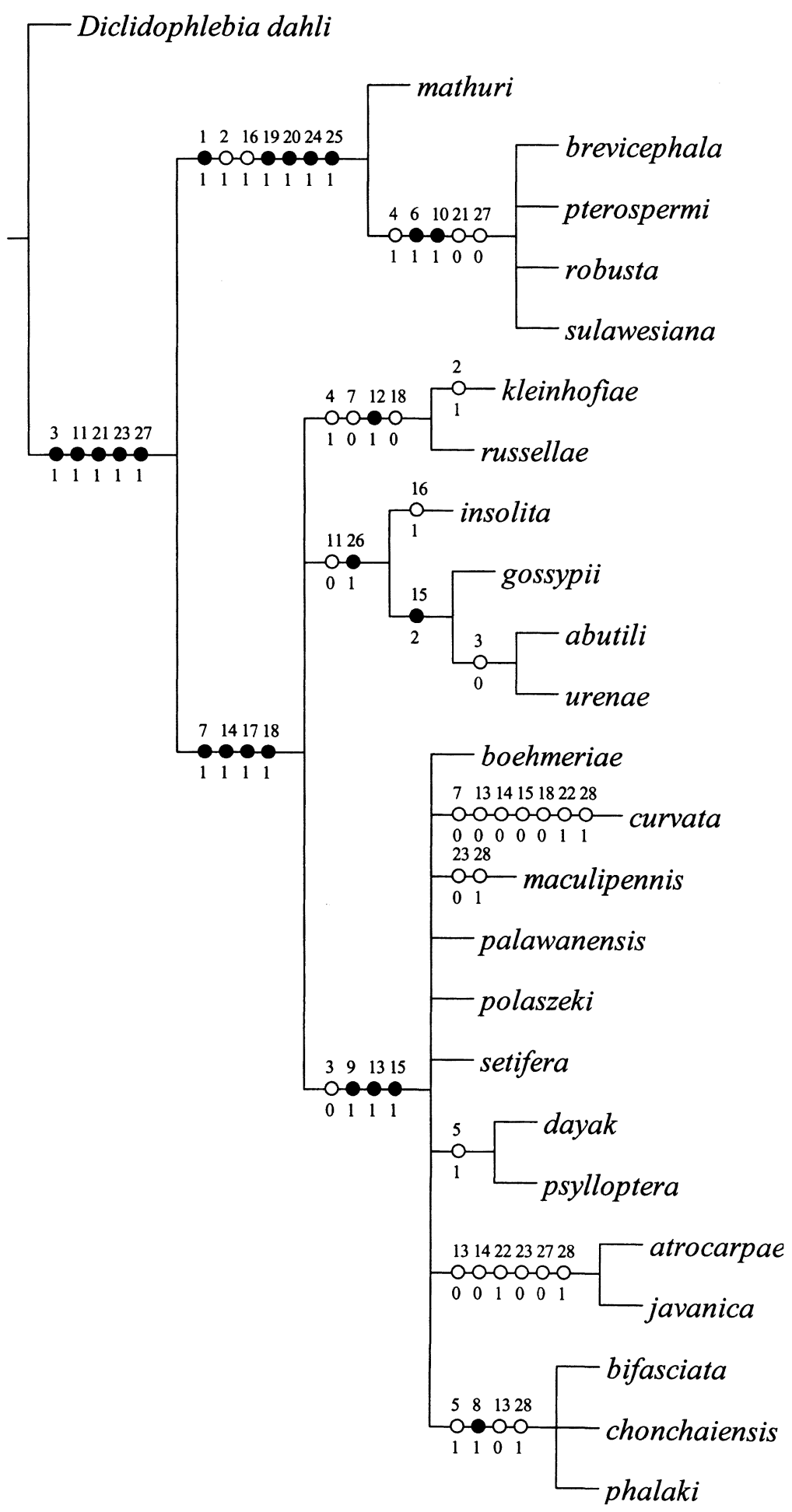

FIG. 48. Cladogram of selected Paurocephala species (adult and larval characters) with Diclidophlebia dahli as outgroup. 
psylloptera-groups. The gossypii-group, on the other hand, is supported by the larval character: marginal sectasetae on caudal plate based on large tubercles (character 26).

Both $P$. psylloptera, the type species of Paurocephala and $P$. chonchaiensis, the type species of Thoracocorna are members of the psylloptera-group. There is, consequently, no phylogenetic justification for Thoracocorna and its synonymy with Paurocephala is confirmed.

\section{Distribution and host plants}

In its revised definition, Paurocephala is restricted to the Old World. The majority of species and species groups occurs in the Indo-Australian region. Except for the basal $P$. lienhardi which occurs in Borneo, the gossypii-group consists of a monophyletic clade of Afrotropical species. This nested pattern suggests a dispersal from the Oriental to the Afrotropical regions rather than a vicariant event between the two.

The present study confirms the narrow host ranges of Paurocephala spp. which was previously shown for $P$. psylloptera, $P$. sauteri and $P$. trematos by Yang et al. (1986). Known host plants of Paurocephala spp. belong to the Malviflorae with the exception of the Afrotropical $P$. insolita which develops on Theiflorae (Theales, Clusiaceae). The brevicephala-, kleinhofiae- and gossypii-groups are associated with hosts of the order Malvales (Malvaceae and Sterculiaceae), whereas the psyllopteragroup is with Urticales (Urticaceae, Moraceae and Ulmaceae).

\section{Acknowledgements}

David Hollis (BMNH) generously shared his knowledge on psyllids with us throughout the project. His comments on an earlier manuscript version substantially improved the paper. For all this we are very grateful to him. The loan or gift of material was vital, and we thank all our colleagues for their kind help: David Hollis, Jon Martin and Andy Polaszek (BMNH), Douglass Miller (USNM), Charles Lienhard and Bernd Hauser (MHNG), Li Fasheng (BAUC), David J. Preston (BPBM), Man-Miao Yang (NCHU), Kouichi Matsumoto (TULE), Ermenegildo Tremblay (UNEI), Yorio Miyatake (OMNH), and Andreas Täger (DEIC). V. V. Ramamurthy provided information on Indian collections. At the Naturhistorisches Museum Basel we thank Stephania Mifsud and Denise Wyniger for their various help. The SEM photographs were prepared at the Labor für Rasterelektronenmikroskopie, Universität Basel and we thank Richard Guggenheim and Daniel Mathys for their precious help. Roland Mühlethaler (Institut NLU, Universität Basel) kindly helped in producing the SEM plates. This work was funded by a grant from the Schweizerischer Nationalfonds, Project No.31-52297.97 (to Daniel H. Burckhardt).

\section{References}

BeKker-Migdisova, E. E., 1973, Systematics of the Psyllomorpha and the position of the group within the order Homoptera, in Iné Narchuk, E.P. (ed.) Doklady na Dvadsat Chetvertom Ezheghodnom Chtenii Pamyati N.A. Kholodkovskogo, 1-2 aprelya 1971 (British Lending Library Translation RTS 8526), pp. 90-117.

Boselli, F. B., 1929, Studii sugli Psyllidi (Homoptera: Psyllidae o Chermidae) II. Descrizione di una nuova specie di Paurocephala della Cina e dei suoi stadii larvali, Bollettino di Zoologia generale ed agraria, 21, 251-264.

Boselli, F. B., 1930, Studii sugli Psyllidi (Homoptera: Psyllidae o Chermidae) VI. Psyllidi di Formosa raccolti dal Dr. R. Takahashi, Bollettino di Zoologia generale ed agraria, 24, 175-210. 
Braza, R. D. and Calilung, V. J., 1981, Some Philippine Psyllids (Psyllidae: Homoptera), The Philippine Entomologist, 4, 319-360.

Brown, R. G. and Hodkinson, I. D., 1988, Taxonomy and Ecology of the Jumping Plant-lice of Panama (Homoptera: Psylloidea), Entomonograph 9 (Leiden: E. J. Brill), 304 pp.

BurckhardT, D., 1996, Two new Paurocephala Species from Paraguay (Hemiptera, Psylloidea), Deutsche Entomologische Zeitschrift, 43(1), 77-82.

Burckhardt, D. and BASSET, Y., 2000, The jumping plant-lice (Hemiptera, Psylloidea) associated with Schinus (Anacardiaceae): systematics, biogeography and host plant relationships, Journal of Natural History, 34, 57-155.

Crawford, D. L., 1914, New genera and species of psyllidae from the Philippine Islands, The Philippine Journal of Science, 8, 293-301, 1 pl.

Crawford, D. L., 1915, Ceylonese and Philippine Psyllidae (Homoptera), The Philippine Journal of Science, 10, 257-269, $1 \mathrm{pl}$.

Crawford, D. L., 1917, Philippine and Asiatic Psyllidae, The Philippine Journal of Science, 12, 163-175, $1 \mathrm{pl}$.

Crawford, D. L. 1919, The jumping plant lice of the Palaeotropics and the South Pacific Islands, The Philippine Journal of Science, 15, 139-207, 3 pls.

Crawford, D. L., 1924, New Indian Psyllidae, Records of the Indian Museum, 26, 615-621.

Crawford, D. L., 1927, Psyllidae (Chermidae). Insects of Samoa and other Samoan terrestrial arthropoda, British Museum (Natural History), 1, 29-33.

Crawford, D. L., 1928, Fauna sumatrensis. (Beitrag Nr. 61). Psyllidae, Entomologische Mitteilungen, 17, 425-426.

Dobreanu, E. and Manolache, C., 1962, Fauna republicii populare Romine, Insecta, VIII(3), 1-376.

Enderlein, G., 1914, H. Sauter's Formosa-Ausbeute: Psyllidae (Homopt.) Psyllidologica II, Entomologische Mitteilungen, 3, 230-235.

FANG, S. J. and YANG, C. T., 1986, Psylloidea of Taiwan (Homoptera: Sternorrhyncha) Supplement, Taiwan Museum Special Publication Series, 6, 119-176.

FarRIS, J. S., 1988, Hennig86, Version 1.5. Published by the author, Port Jefferson, New York.

Heslop-Harrison, G., 1951, Subfamily separation in the Homopterous Psyllidae. II, Annals and Magazine of Natural History (series 12), 4, 1-35.

HeSLOP-HARrison, G., 1958, Subfamily separation in the homopterous Psyllidae. III (a-c), Annals and Magazine of Natural History (series 13), 1, 561-579.

Hodkinson, I. D., 1983, The psyllids (Homoptera: Psylloidea) of the Austro-Oriental, Pacific and Hawaiian zoogeographical realms: an annotated check list, Journal of Natural History, 17, 341-377.

Hodkinson, I. D., 1986, The psyllids (Homoptera: Psylloidea) of the Oriental Zoological Region: an annotated check-list, Journal of Natural History, 20, 299-357

Hollis, D., 1976, Jumping plant lice of the tribe Ciriacremini (Homoptera:Psylloidea) in the Ethiopian region, Bulletin of the British Museum (Natural History) (Entomology), 34(1), 1-83.

Hollis, D., 1984, Afrotropical jumping plant lice of the family Triozidae (Homoptera: Psylloidea), Bulletin of the British Museum (Natural History) (Entomology), 49, 1-102.

Hollis, D. and Broomfield, P. S., 1989, Ficus-feeding psyllids (Homoptera), with special reference to the Homotomidae, Bulletin of the British Museum (Natural History) (Entomology), 58, 131-183.

Kandasamy, C., 1986, Taxonomy of South Indian Psyllids, Records of the Zoological Survey of India, 84, iii + $110 \mathrm{pp}, 1 \mathrm{pl}$.

Klimaszewski, S. M., 1964, Studies on systematics of the suborder Psyllodea, Annales Zoologici, 22(5), 1-58.

KlimaszewsKI, S. M., 1970, Psyllidologische Notizen XVIII-XX (Homoptera), Annales Zoologici, 27, 417-427.

Kuwayama, S., 1922, A list of the known species of Japanese Psyllidae, Insect World, 26, 368.

Kuwayama, S., 1931, A revision of the Psyllidae of Taiwan, Insecta Matsumurana, 5, 117-133.

Kuwayama, S., 1943, On some species of Psyllidae from Amami-Osima and Yakusima, Transactions of the Natural History Society of Formosa, 33, 504-511.

LaING, F., 1922, On some Fijian Psyllidae (Homoptera), Annals and Magazine of Natural History, 9(9), 553-557. 
LI, F. and YANG, C. K., 1987, Six new species of Stenopsylla of the family Triozidae and a new genus of the family Carsidaridae from China (Homoptera: Psylloidea), Wuyi Science Journal, 7(12), 27-38.

Loginova, M. M., 1972, Revision of the jumping plant lice of the tribe Pauropsyllini Crawf. (Homoptera, Psylloidea, Carsidaridae), Entomologicheskoe Obozrenie, 51, 837-853.

Mathur, R. N., 1975, Psyllidae of the Indian Subcontinent (New Delhi: Indian Council of Agricultural Research), xii $+429 \mathrm{pp}$.

MiYatake, Y., 1964, On the Psyllidae from the Yaeyama Group, the Ryukyus (Hemiptera: Homoptera), Reports of the Committee on Foreign Scientific Research, Kyushu University, No. 2 (Second Report of the Kyushu University Expedition to the Yaeyama Group, Ryukyus), 121-132.

Miyatake, Y., 1965, Notes on the Psyllidae from the Ryukyu Islands (Hemiptera: Homoptera), Kontyû, 33, 171-189.

MiyataKe, Y., 1971, Studies on the Philippine Psyllidae (Hemiptera: Homoptera) I. Results of the Natural History Survey by Osaka Museum of Natural History and National Museum of the Philippines, 1969. Part I, Bulletin of the Osaka Museum of Natural History, 25, 51-60.

Navasero, M. V. and Calilung, V. J., 1998, The Psylloids (Hemiptera: Psylloidea) of the Philippines: an annotated checklist, The Philippine Entomologist, 12(1), 13-28.

Nixon, K. C. (1999), WINCLADA. Shareware download at cladistics.com.

Ossiannilsson, F., 1992, The Psylloidea (Homoptera) of Fennoscandia and Denmark, Fauna Entomologica Scandinavica, 26, 1-347.

Pettey, F. W., 1924, South African psyllids, Entomology Memoirs, 2, 21-30.

Pettey, F. W., 1933, New species of South African Psyllids III, Entomology Memoirs, 8, 3-23.

Ramakrishna Ayyar, T. V., 1924, List of Psyllidae recorded from India and Ceylon, Records of the Indian Museum, 26, 621-625.

Russell, L. M., 1943, An apparently new species of Paurocephala Crawford (Homoptera, Psyllidae, Pauropsyllinae), Proceedings of the Entomological Society of Washington, 45, 115-120.

Russell, L. M., 1946, A new African species of Paurocephala Crawford (Homoptera: Psyllidae: Pauropsyllinae), Journal of the Washington Academy of Sciences, 36, 94-97.

SASAKI, K., 1954, A list of known species and their host-plants of the Psyllidae of Japan (Homoptera), Scientific Reports of the Matsuyama Agricultural College, 14, 29-39.

TAKahashi, R., 1936, Food habits and new habitats of Formosan Psyllidae, with notes on the peculiar food habits of Formosan phytophagus insects, Kontyû, 10, 291-296.

Uichanco, L. B., 1921, New records and species of Psyllidae from the Philippines Islands, with descriptions of some preadult stages and habits, The Philippine Journal of Science, 18, 259-288, 5 pls.

UYE, T., 1937, Some jumping plant-lice of Kyushu, Entomological World, 5, 605-608.

VONDRÁČEK, K., 1952, Results of Zoological scientific expedition of the national museum in Praha to Turkey, Acta Entomologica Musei Nationalis Pragae, XXVIII(422), 435-450.

VondráČEK, K., 1957, Mery-Psylloidea. Fauna CSSR, 9 (Praha), 431 pp. (in Czech).

VONDRÁČEK, K., 1963, Jumping plant-lice (Psylloidea-Homoptera) of Central Africa Part I (Congo), Acta Entomologica Musei Nationalis Pragae, 35, 263-290.

White, I. M. and Hodkinson, I. D., 1985, Nymphal taxonomy and systematics of the Psylloidea (Homoptera), Bulletin of the British Museum of Natural History (Entomology), 50, 153-301.

YANG, C. K. and LI, F. S., 1987, Six new species and a new genus of Paurocephalinae (Psyllidae: Homoptera), Wuyi Science Journal, 6, 45-58.

YANG, C. T., 1984, Psyllidae of Taiwan, Taiwan Museum Special Publication Series, 3, 1-305.

YANG, M. M., YANG, C. T. and ChaO, J. T., 1986, Reproductive isolation and taxonomy of two Taiwanese Paurocephala species (Homoptera: Psylloidea), Taiwan Museum Special Publication Series, 6, 177-203. 